\title{
The identification and characterization of Mio10 and MINOS1 as novel regulators of mitochondrial inner membrane organization
}

\author{
Ph.D. Thesis \\ in partial fulfilment of the requirements \\ for the degree "Doctor of Philosophy (Ph.D.)" \\ in the Molecular Biology graduate program \\ at the Georg August University Göttingen, \\ Faculty of Biology
}

\section{Submitted by \\ Alwaleed Alkhaja}

born in

Manama, Bahrain 


\section{Thesis Committee Members:}

\section{Prof. Dr. Peter Rehling}

Department of Biochemistry II

University of Göttingen

Göttingen

\section{Prof. Dr. Reinhard Jahn}

Department of Neurobiology

Max-Planck-Institute for Biophysical Chemistry

Göttingen

Prof. Dr Markus Zweckstetter

Department for NMR-Based Structural Biology

Max-Planck-Institute for Biophysical Chemistry Göttingen

Date of thesis submission: $31 / 03 / 2012$

Date of oral examination: 02/05/2012 


\section{Part of this thesis has been previously published:}

Alkhaja, A. K., Jans, D. C, Nikolov, M., Vukotic, M., Lytovchenko, O., Ludewig, F., Schliebs, W., Riedel, D., Urlaub, H., Jakobs, S., and Deckers M. (2012). MINOS1 is a conserved component of mitofilin complexes and required for mitochondrial function and cristae organization. Mol. Biol. Cell. 23, 247-57.

I hereby declare that this $\mathrm{PhD}$ thesis entitled "The identification and characterization of Mio10 and MINOS1 as novel regulators of mitochondrial inner membrane organization" has been written independently with no other aids or sources than quoted.

Alwaleed Alkhaja

Göttingen, 30.03.12 
to my parents 


\section{TABLE OF CONTENTS}

TABLE OF CONTENTS

LIST OF FIGURES

LIST OF TABLES

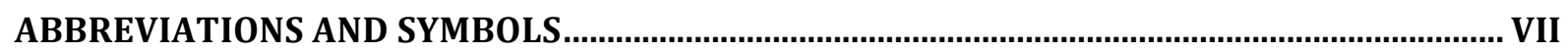

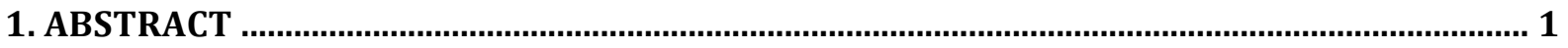

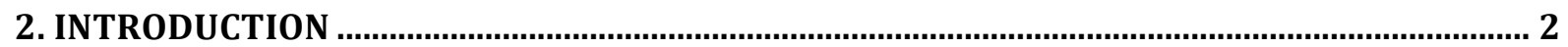

2.1 DUAL GENOMIC MITOCHONDRIAL PROTEOME

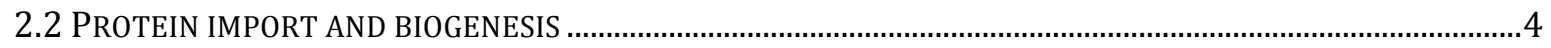

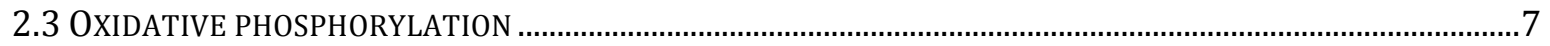

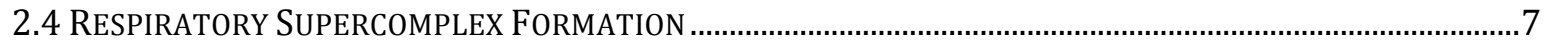

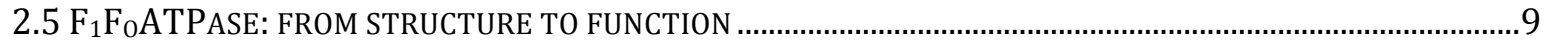

2.6 HIGH ORDERED ORGANIZATION OF THE $\mathrm{F}_{1} \mathrm{~F}_{0}$ ATPASE: FROM DIMERS TO OLIGOMERS.............................. 10

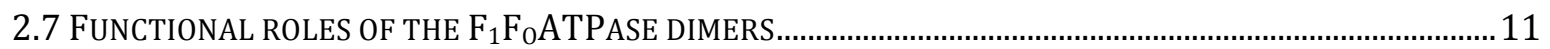

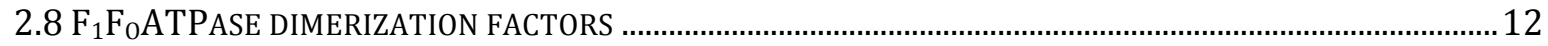

$2.9 \mathrm{~F}_{1} \mathrm{~F}_{0}$ ATPASE DIMERIZATION AND MAINTENANCE OF CRISTAE MORPHOLOGY …....................................... 14

2.10 THE INNER MEMBRANE AND CRISTAE MEMBRANE ARCHITECTURE ……..............................................15

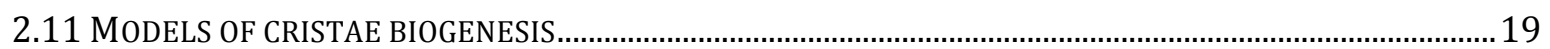

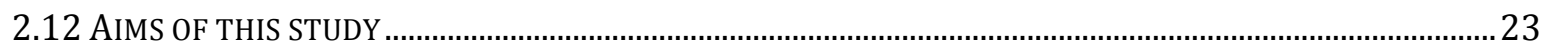

3. MATERIALS AND METHODS

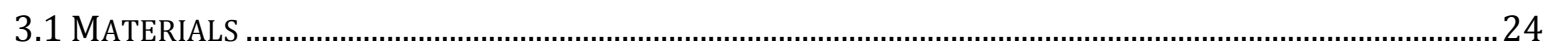

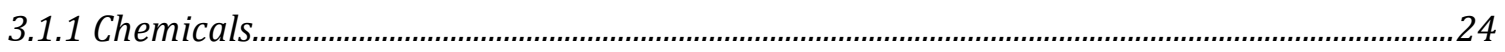

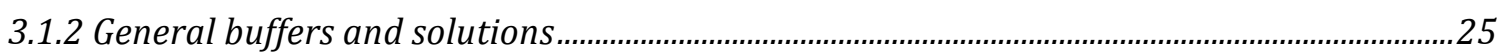

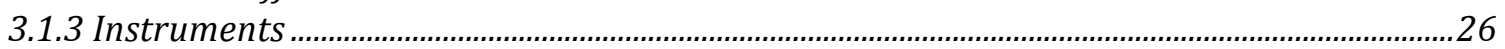

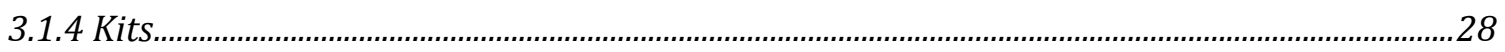

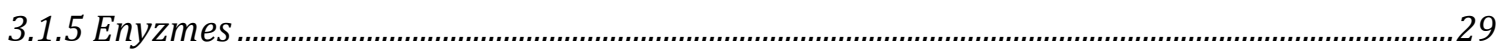

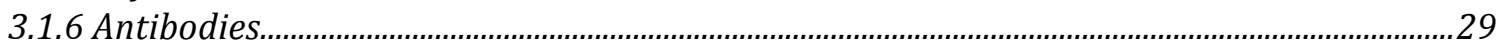

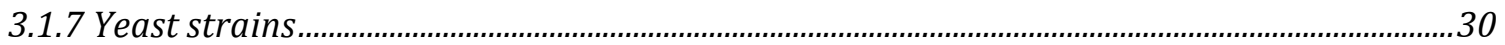

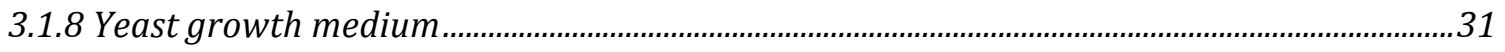

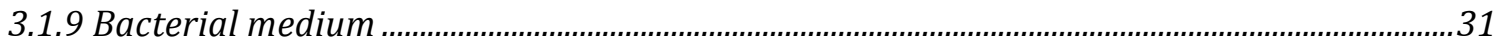

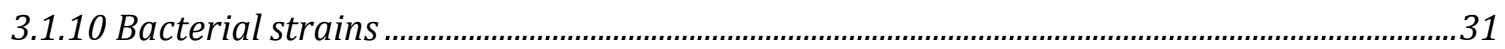

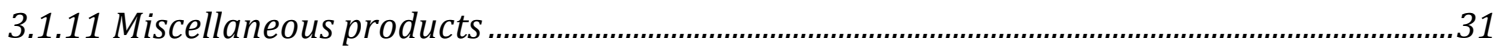

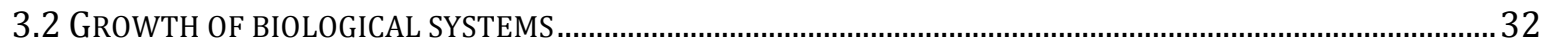

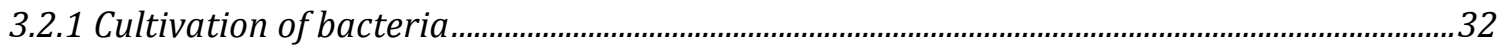

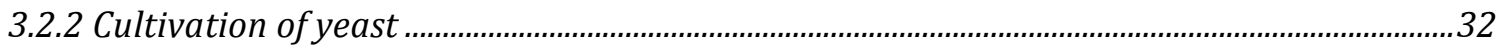

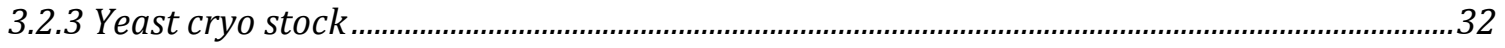

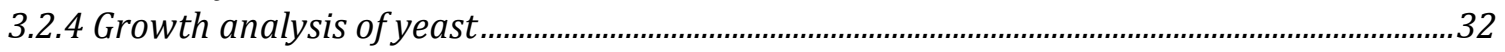

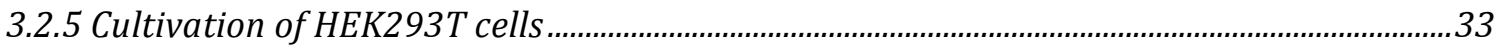

3.3 MoleCULAR BIOLOGY TECHNIQUES ………................................................................................... 33

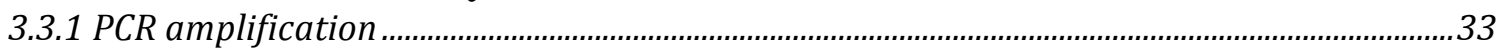

3.3.2 Determination of nucleic acid concentrations ...........................................................................33

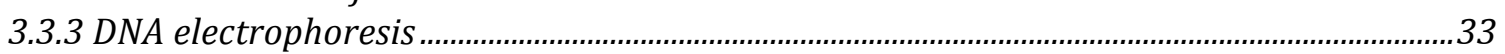

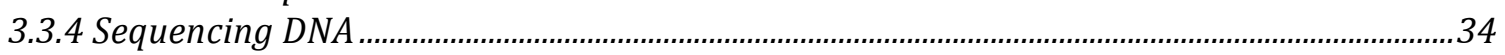

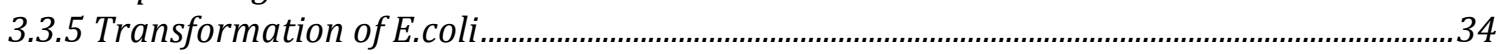

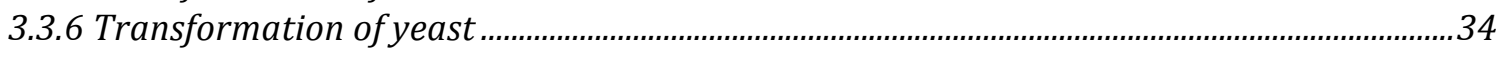

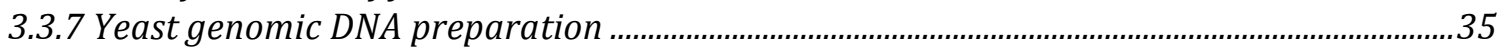

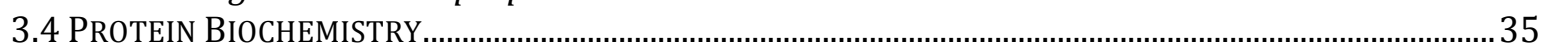

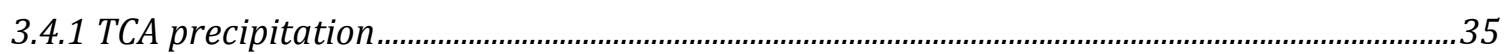

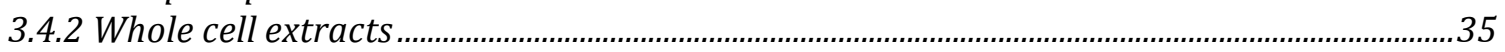

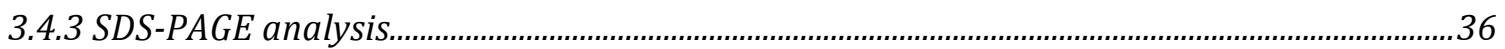




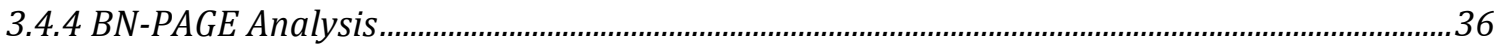

3.4.4.1 First Dimension BN-PAGE ………………………………………………………………………………………………………...... 36

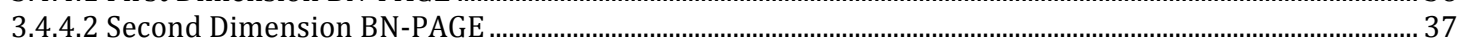

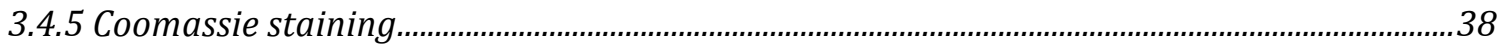

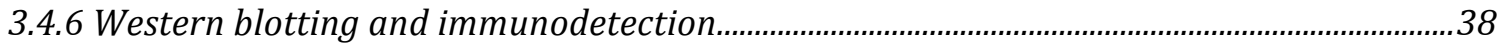

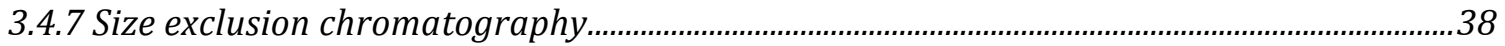

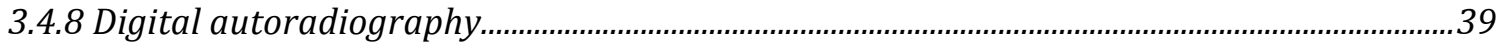

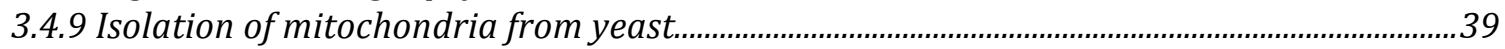

3.4.10 Isolation of mitochondria from HEK 293T cells ................................................................ 40

3.4.11 Labeling of precursor proteins with [35S] methionine ............................................................40

3.4.12 Import and assembly of radiolabeled precursor protein ........................................................... 41

3.4.12.1 Import into yeast isolated mitochondria.......................................................................................................................... 41

3.4.12.2 Import into HEK293T isolated mitochondria ........................................................................................................ 41

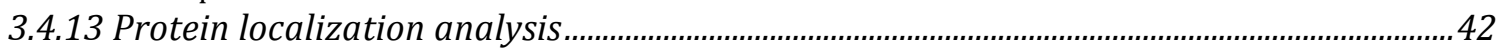

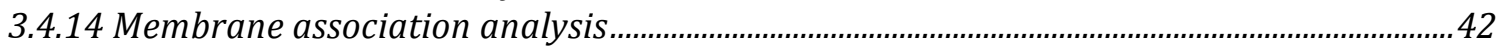

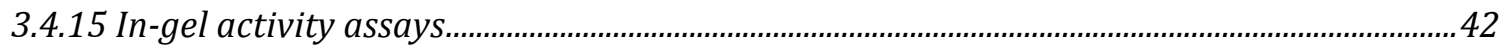

3.4.16 Protein complex isolation by immunoprecipitation ……....................................................... 43

3.4.17 Crosslining antibodies for co-immunoprecipitations .................................................................44

3.4.18 Co-immunoprecipitation from isolated yeast mitochondria ....................................................4

3.4.19 Co-immunoprecipitation from HEK293T cells ........................................................................45

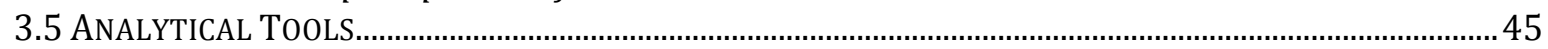

3.5.1 In silico analysis and multiple sequence alignments................................................................... 45

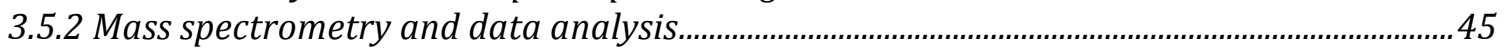

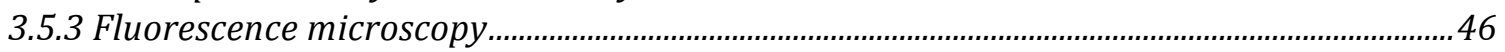

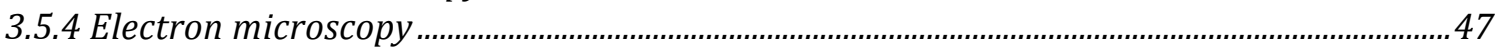

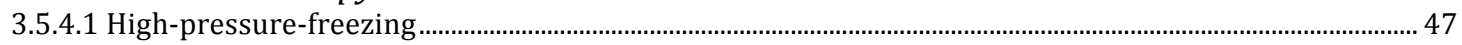

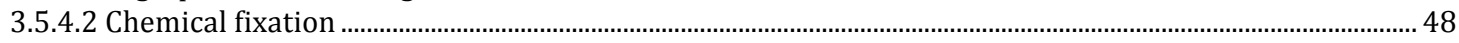

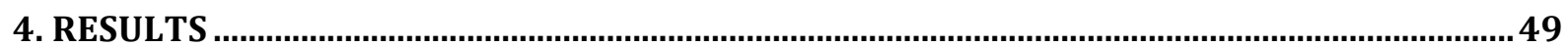

4.1 IDENTIFICATION OF A PUTATIVE $\mathrm{F}_{1} \mathrm{~F}_{0}$ ATPASE DIMERIZATION FACTOR IN HIGHER EUKARYOTES $(H$.

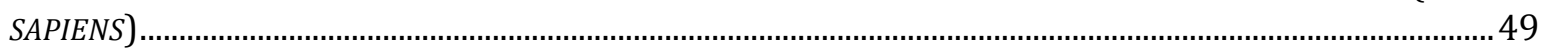

4.1.1 in silico analysis: identification of Mio10 and MINOS1 .........................................................49

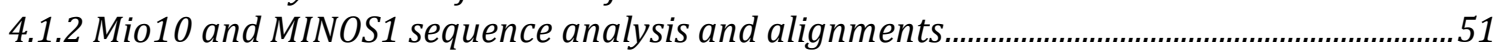

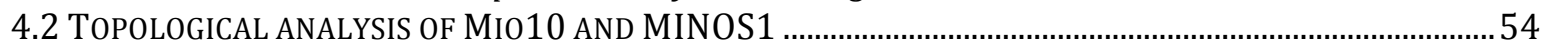

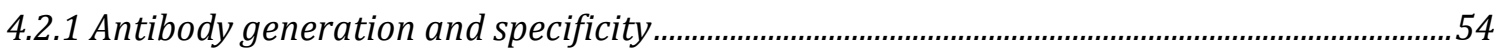

4.2.2 Topological analysis of Mio10................................................................................................ 55

4.2.3 Subcellular localization of MINOS1 by immunofluorescence ..................................................56

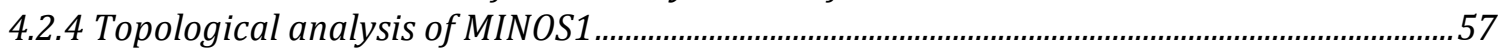

4.3 ANALYSIS OF MIO10 AND MINOS1 ASSOCIATION WITH THE F F FoATPASE .............................................59

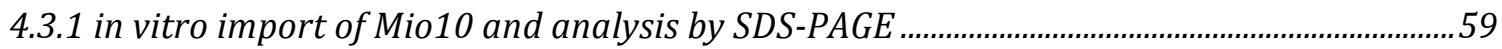

4.3.2 Mio10 and MINOS1 assembly analysis by BN-PAGE ……....................................................60

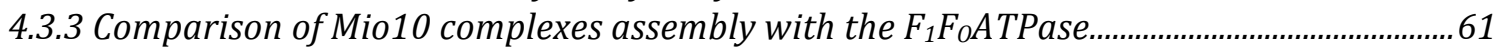

4.3.4 Analysis of Mio10 assembly in the absence of $F_{1} F_{0} A T P a s e$ dimers .........................................63

4.3.5 Second dimension analysis of Mio10 complexes in atp204 mitochondria .............................65

4.3.6 Biochemical isolation of $F_{1} F_{0} A T P a s e$ components....................................................................66

4.4 MIO1O DELETION MUTANT ANALYSIS ………………...............................................................................67

4.4.1 Growth analysis of MIO10 deletion mutant mitochondria ......................................................67

4.4.2 Analysis of respiratory chain complexes in mio104 .................................................................6

4.4.3 Assessment of $F_{1} F_{0} A T P a s e$ activity and oligomerization in mio104 mutants ......................69

4.5 IDENTIFICATION OF MIO10 AND MINOS1 INTERACTION PARTNERS..................................................... 71

4.5.1 Identification of Mio10 interaction partners............................................................................

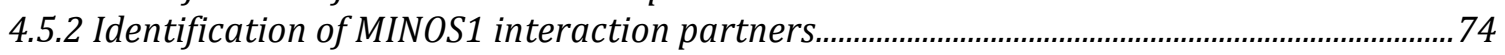

4.6 CHARACTERIZATION OF MIO10 AND MINOS1 INTERACTION WITH FCJ1 AND MITOFILIN .......................76

4.7 INVESTIGATION OF THE ROLE OF MIO10 IN MITOCHONDRIAL MORPHOLOGY ……..................................... 78

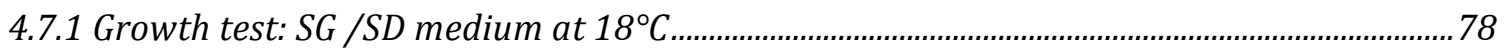

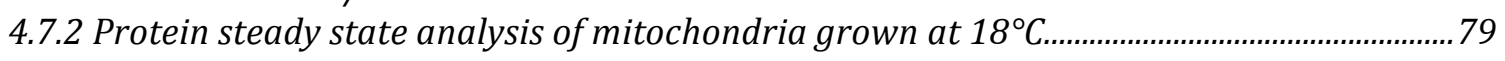

4.7.3 Live-cell fluorescence microscopy analysis of mio104 mitochondria.......................................80 


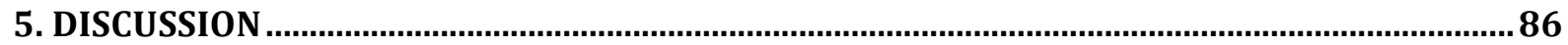

5.1 MIO10 AND MINOS1 ARE NOT F $F_{0}$ ATPASE DIMERIZATION FACTORS ................................................. 86

5.1.1 Identification of the inner membrane proteins: Mio10 and MINOS1 ….................................86

5.1.2 Mio10 and MINOS1 complexes comigrate but not physically associate with the

$F_{1} F_{0}$ ATPase

5.1.3 Mio10 and MINOS1 are not stably associated with the $F_{1} F_{0}$ ATPase......................................89

5.1.4 Mio10 does not affect $F_{1} F_{0} A T P a s e$ activity or oligomerization.................................................90

$5.1 .5 F_{1} F_{0}$ ATPase dimerization in higher eukaryotes ...................................................................... 91

5.2 THE MINOS COMPLEX: MITOCHONDRIAL INNER MEMBRANE ORGANIZING SYSTEM COMPLEX................92

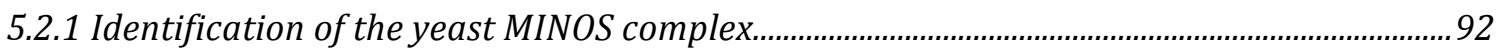

5.2.2 Identification of the human MINOS complex..................................................................................

5.2.3 Mio10 and MINOS1 form a large complex (the MINOS complex) ..........................................95

5.2.4 The identification of additional MINOS components.............................................................97

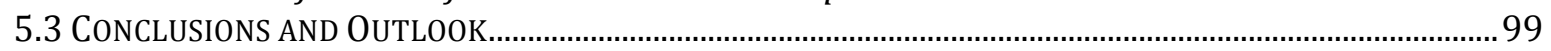

6. REFERENCES

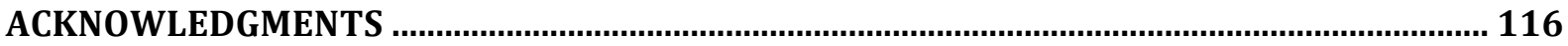

CURRICULUM VITAE 


\section{LIST OF FIGURES}

Figure 1 Overview of various mitochondrial import pathways

Figure 2 Overview of mitochondrial respiratory chain and oxidative phosphorylation.

Figure 3 Structural organization of respiratory chain supercomplexes in yeast

Figure 4 Nomenclature and organization of $\mathrm{F}_{1} \mathrm{~F}_{\mathrm{O}} \mathrm{ATPase}$ subunits in yeast and H.sapiens.

Figure 5 Stepwise assembly of the $\mathrm{F}_{1} \mathrm{~F}_{\mathrm{O}} \mathrm{ATPase}$ dimer

Figure 6 Aberrant mitochondrial morphology in ATP20 and ATP21 deletion mutants

Figure 7 Overview of factors involved in cristae biogenesis and maintenance

Figure 8 Hypothetical models of cristae biogenesis

Figure 9 Identification of Mio10 and MINOS1 by in silico analysis

Figure 10 Molecular organization of selected proteins

Figure 11 Alignment of Mio10 yeast homologs

Figure 12 Alignment of MINOS1 homologs

Figure 13 Antibody specificity test

Figure 14 Mio10 is a mitochondrial IMS protein

Figure 15 MINOS1 is a mitochondrial localized protein

Figure 16 MINOS1 is a mitochondrial IMS protein

Figure 17 Topology of Mio10 and MINOS1

Figure 18 Import of Mio10 into mitochondria is membrane potential independent

Figure 19 Mio10 and MINOS1 assemble into high molecular sized mitochondrial complexes

Figure 20 Mio10 comigrates with $\mathrm{F}_{1} \mathrm{~F}_{\mathrm{O}} \mathrm{ATPase}$ complexes

Figure 21 Mio10 assembly requires the presence of Atp20

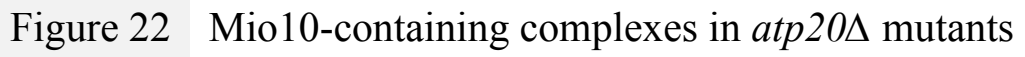


Figure 23 Mio10 and MINOS1 are not stably associated with the $\mathrm{F}_{1} \mathrm{~F}_{\mathrm{O}} \mathrm{ATPase}$

Figure 24 Mio10 is not required for mitochondrial respiration or supercomplex assembly

Figure $25 \quad \mathrm{~F}_{1} \mathrm{~F}_{\mathrm{O}} \mathrm{ATPase}$ activity and oligomerization are not affected in mio $10 \Delta$ mutants

Figure 26 Mio10 interacts with Fcj1

Figure 27 Schematic of SILAC approach and mass spectrometric analysis

Figure 28 MINOS1 interacts with Mitofilin

Figure 29 Mio10/Fcj1 and MINOS1/Mitofilin form large mitochondrial complexes

Figure $30 \mathrm{MIO} 10$ and $F C J 1$ deletion mutants' growth defect at low temperatures

Figure 31 Protein steady-state levels are unaffected in mio $10 \Delta$ mutants

Figure 32 Mitochondrial morphology is altered in miol04

Figure 33 Aberrant cristae morphology in mio10s mitochondria

Figure 34 Absence of apparent cristae and cristae tips in mio10 0 mitochondria

Figure 35 MINOS complex is critical for cristae biogenesis

Figure 36 MINOS is a multifunctional protein

Figure 37 Overview of MINOS roles in cristae biogenesis 


\section{LIST OF TABLES}

Table 1 Details of various antibodies used in this study

Table 2 Details of yeast strains used in this study including genotype and source

Table 3 SDS-PAGE pipetting scheme

Table 4 BN-PAGE pipetting scheme

Table 5 Composition of in-gel activity assay buffers

Table 6 Experimental conditions for various immunoprecipitation assays

Table 7 Identified proteins of the Mio $10^{\mathrm{SF}}$ StrepTactin-chromotography

Table 8 The MINOS components as identified by various studies 


\section{ABBREVIATIONS AND SYMBOLS}

\begin{tabular}{|c|c|}
\hline${ }^{\circ} \mathrm{C}$ & Degrees centigrade \\
\hline$\%$ & Percent \\
\hline$\leq$ & Less than or equal to \\
\hline ADP & Adenosine diphosphate \\
\hline AIM & $\begin{array}{l}\text { Altered inheritance of } \\
\text { mitochondria }\end{array}$ \\
\hline ATP & Adenosine triphosphate \\
\hline ATPase & ATP synthase \\
\hline APS & Ammoniumpersulsulfate \\
\hline AVO & $\begin{array}{l}\text { Antimycin, Valinomycin, } \\
\text { Oligomycin }\end{array}$ \\
\hline $\mathrm{BN}$ & Blue Native \\
\hline BSA & Bovine serum albumin \\
\hline C-terminus & Carboxyl terminus \\
\hline $\mathrm{CK}$ & Creatine Kinase \\
\hline $\mathrm{CP}$ & Creatine Phosphate \\
\hline $\mathrm{COX}$ & Cytochrome c oxidase \\
\hline Complex I & NADH dehydrogenase \\
\hline $\begin{array}{r}\text { Complex } \\
\text { III }\end{array}$ & Cytochrome bc1 complex \\
\hline $\mathrm{CN}$ & Clear native \\
\hline CSM & Complete Supplement Mixture \\
\hline $\mathrm{dH}_{2} \mathrm{O}$ & Distilled water \\
\hline DAB & 3,3' Diaminobenzidine \\
\hline DMP & $\begin{array}{l}\text { Dimethyl pimelimidate } \\
\text { dihydrochloride }\end{array}$ \\
\hline DMSO & Dimethylsulfoxide \\
\hline DMEM & $\begin{array}{l}\text { Dulbecco's Modified Eagle } \\
\text { Medium }\end{array}$ \\
\hline DNA & Deoxyribonucleic acid \\
\hline DNase & Deoxyribonuclease \\
\hline DTT & Dithioreitol \\
\hline E-value & Expect value \\
\hline E.coli & Escherichia coli \\
\hline ECL & Enhanced chemiluminescence \\
\hline EDTA & $\begin{array}{l}\text { Ethylene diamine tetraacetic } \\
\text { acid }\end{array}$ \\
\hline $\mathrm{FADH}_{2}$ & Flavin adenine dinucleotide \\
\hline Fcj1 & Formation of cristae junction 1 \\
\hline $\mathrm{H}+$ & Hydrogen ions \\
\hline H.sapiens & Homo Sapiens \\
\hline $\mathrm{H} / \mathrm{L}$ & High over low \\
\hline $\mathrm{HCl}$ & Hydchloric acid \\
\hline HPF & High pressure freezing \\
\hline Hrs & Hours \\
\hline IBM & Innerboundary membrane \\
\hline IgG & Immunoglobulin G \\
\hline IMMT & Mitofilin \\
\hline
\end{tabular}

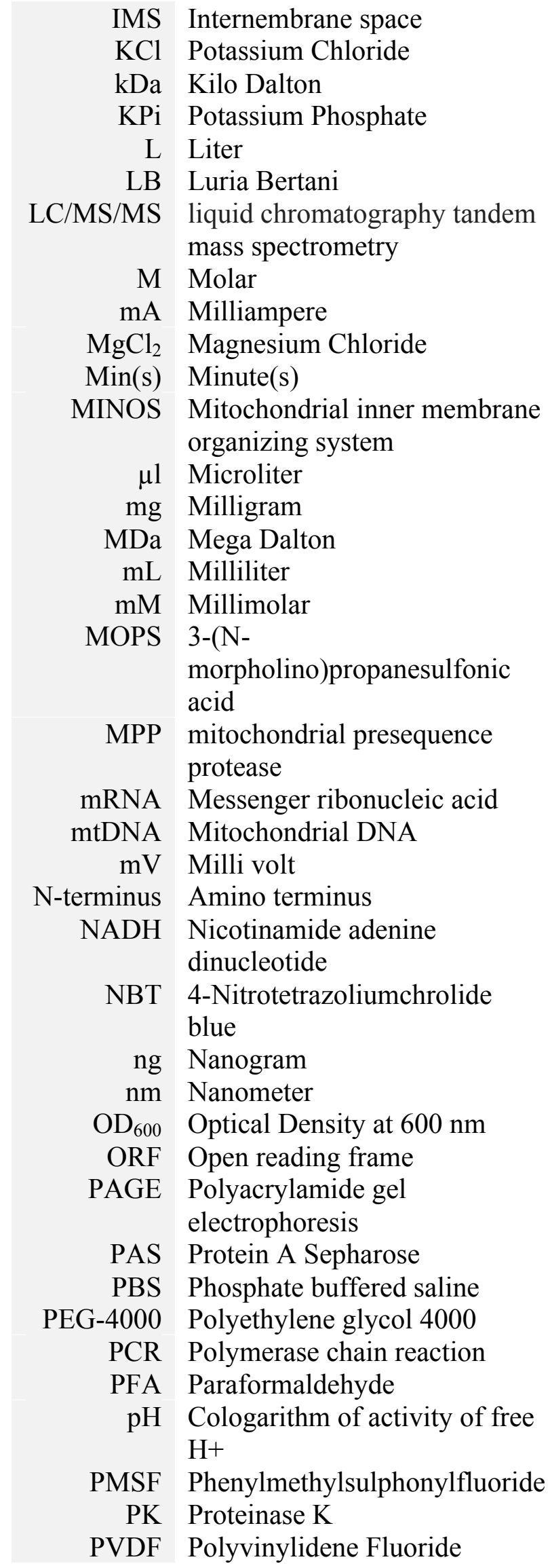




\begin{tabular}{|c|c|}
\hline $\mathrm{rpm}$ & Revolutions per minute \\
\hline RNA & Ribonucleic acid \\
\hline RNAi & RNA interference \\
\hline RT & Room temperature \\
\hline SD & Synthetic glucose \\
\hline SDS & Sodium dodecyle sulfate \\
\hline SGD & Saccharomyces Genome \\
\hline & Database \\
\hline SG & Synthetic glycerol \\
\hline SEM & Sucorse EDTA MOPS \\
\hline SILAC & $\begin{array}{l}\text { Stable isotope labeling of } \\
\text { amino acids in cell culture }\end{array}$ \\
\hline S.cerevisiae & Saccharomyces cerevisiae \\
\hline SF & Streptavidin FLAG \\
\hline Taq & Thermus aquaticus \\
\hline TAE & Tris-acetate-EDTA \\
\hline TBS & Tris buffered saline \\
\hline TBST & Tris buffered saline tween \\
\hline TCA & Trifluoroacetic acid \\
\hline TEMED & 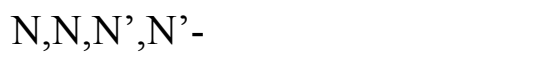 \\
\hline & Tetramethylethylene diamine \\
\hline TIM & Translocase of inner membrane \\
\hline $\mathrm{TM}$ & Transmembrane \\
\hline TOM & Translocase of outer membrane \\
\hline TX-100 & Triton X-100 \\
\hline Tris & $\begin{array}{l}\text { Tris(hydroxymethly)- } \\
\text { aminomethane }\end{array}$ \\
\hline URA & Uracil \\
\hline $\mathrm{V}$ & Volt \\
\hline $\mathrm{v} / \mathrm{v}$ & Volume per volume \\
\hline Vero & $\begin{array}{l}\text { African green monkey } \\
\text { epithelial cells }\end{array}$ \\
\hline $\mathrm{w} / \mathrm{v}$ & Weight per volume \\
\hline YPD & $\begin{array}{l}\text { Yeast extract, peptone, } \\
\text { dextrose }\end{array}$ \\
\hline YPG & $\begin{array}{l}\text { Yeast extract, peptone, } \\
\text { glycerol }\end{array}$ \\
\hline YPS & Yeast extract, peptone, sucrose \\
\hline $\mathrm{ZZ}$ & Protein-A \\
\hline
\end{tabular}




\section{ABSTRACT}

Various mitochondrial inner membrane proteins contribute to the structure of cristae membranes and to the overall dynamic morphology of the inner membrane. These proteins include the $\mathrm{F}_{1} \mathrm{~F}_{\mathrm{O}} \mathrm{ATPase}$ dimers, which contribute to the curvature and angular shape of cristae. Also, Fcj1/Mitofilin are involved in cristae junction formation and act in an antagonistic manner in inner membrane curvature with respect to $F_{1} F_{O} A T P a s e$ dimers. Whereas several small proteins mediate $\mathrm{F}_{1} \mathrm{~F}_{\mathrm{O}} \mathrm{ATPase}$ dimerization in yeast by binding sequentially at monomer interfaces, the exact nature of what promotes $\mathrm{F}_{1} \mathrm{~F}_{\mathrm{O}} \mathrm{ATPa}$ e dimerization in higher eukaryotes is yet to be determined. To this end, the initial aim of this study was to identify novel $\mathrm{F}_{1} \mathrm{~F}_{\mathrm{O}} \mathrm{ATPase}$ dimerization factors in higher eukaryotes that parallel the action of small dimerization factors in yeast. An in silico approach to identify novel $\mathrm{F}_{1} \mathrm{~F}_{\mathrm{O}} \mathrm{ATPase}$ dimerization factors resulted in the identification of the human mitochondrial protein MINOS1 as a potential candidate. MINOS1, along with its human homolog, Mio10, were investigated. These proteins however were found not to be stably associated with the $\mathrm{F}_{1} \mathrm{~F}_{\mathrm{O}} \mathrm{ATPase}$. Moreover, Mio10 did not affect $\mathrm{F}_{1} \mathrm{~F}_{\mathrm{O}} \mathrm{ATPase}$ dimerization, oligomerization, or enzymatic activity. Thus a role of Mio10 and MINOS in dimerization was excluded. Instead by using an affinity purification based mass spectrometric analysis, Mio10/MINOS1 were identified as novel interaction partners of Fcj1/Mitofilin. Topological analysis of Mio10 and MINOS together with results of size exclusion chromatography indicated that the two proteins form large mitochondrial inner membrane complexes. Furthermore, analysis of a MIO10 deletion strain by fluorescence and electron microscopy provided evidence for the critical role that Mio10 plays in inner membrane organization. Mitochondria from miol0s and $f_{c j} 1 \Delta$ strains exhibited leaflet-like stacked cristae membranes and appeared to have lost defined cristae tips. The complex that contains Mio10/Fcj1 in yeast and MINOS1/Mitofilin in human mitochondria was hence termed as the MINOS complex (Mitochondrial Inner membrane Organizing System). The presented findings highlight the functional and evolutionary significance of the MINOS complex as a player in mitochondrial inner membrane architecture as well as a multifunctional component in eukaryotic mitochondria and potentially in other cellular organelles. 


\section{INTRODUCTION}

Ancient. Versatile. Dynamic. Mitochondria are eukaryotic organelles of $\alpha$ proteobacterial origins, possessing their own genome as well as having a protein translational machinery (Gray et al., 1999; Fontanesi et al., 2006; Mick et al., 2011). The mitochondrial proteome is of dual genomic origin: a mosaic of mitochondrial and nuclear encoded proteins. Besides their accustomed role in ATP synthesis by oxidative phosphorylation, mitochondria are also involved in cellular metabolism of amino acids, lipids and iron-sulfur clusters as well as playing a role in aging and disease exemplified by their central roles in apoptosis and production of reactive oxygen species (Scorrano et al., 2002; Pan, 2011).

Mitochondria are highly compartmentalized, due to their enclosure by two specialized membranes: the outer and inner membrane. The inner membrane, which surrounds the mitochondrial matrix, can be characterized by two distinguishable regions: the inner boundary membrane (IBM), consisting of membrane stretches adjacent to the outer membrane, and cristae membranes, which are tubular structured invaginations of the inner membrane that extend into the mitochondrial matrix (Zick et al., 2009). Cristae membranes increase the inner membrane surface-to-volume ratio (Frey and Mannella, 2000). They also promote the formation of a diffusion barrier between the intermembrane space (IMS) and the intracristal space, leading to various bioenergetic consequences, such as the regulation of oxidative phosphorylation by limiting diffusion of metabolites like protons and ADP (Mannella et al., 2001). Furthermore, it appears that inner membrane complexes localize differentially: with the respiratory chain and mitochondrial translational machinery residing in the cristae membranes, and the protein import machinery primarily localized at the IBM (Vogel et al., 2006). In addition, electron micrographs also revealed that cristae membranes are attached to the IBM by narrow openings termed cristae junctions, which appear to be constant in size and shape in various organisms (Frey and Mannella, 2000).

Mitochondrial morphology is dynamic and characterized by variable shapes and sizes of often interconnected networks; these networks can vary from simple tubular networks to more branched elaborate networks (Visser et al., 1995; Egner et 
al., 2002; Okamoto and Shaw, 2005). The nature of the mitochondrial network is dependent on opposing processes of mitochondrial fusion and fission (Okamoto and Shaw, 2005; Hoppins et al., 2007). Moreover, some outer membrane proteins, such as Mmm1, Mdm10, and Mdm12 influence mitochondrial morphology (Okomoto and Shaw, 2005). However it appears that inner membrane proteins, such as the $\mathrm{F}_{1} \mathrm{~F}_{\mathrm{O}}$ ATP synthase $\left(\mathrm{F}_{1} \mathrm{~F}_{\mathrm{O}} \mathrm{ATPase}\right), \mathrm{OPA} 1$, and Mitofilin play a more critical role in determining inner membrane architecture and the biogenesis of cristae membranes. The following section reviews the determinants of mitochondrial morphology from its dual-genomic respiratory chain complexes to inner membrane proteins that regulate cristae biogenesis and architecture.

\subsection{Dual genomic mitochondrial proteome}

Phylogenetic analysis and reconstructions based on mitochondrial-encoded proteins such as subunits of the cytochrome $c$ oxidase, have illustrated that contemporary mitochondria have originated from an ancestral endiosymbiot of $\alpha$-proteobacterial origin (Sicheritz-Ponten et al., 1998; Gray et al., 1999; Gray, 2011). Acquisition of the bacterium by early eukaryotic cells is hypothesized to have occurred approximately two billion years ago, a time point in which an environmental global event led to a dramatic increase in atmospheric oxygen levels (Holland, 1994). The symbiotic relationship between the eukaryote and the bacterium, which has potential for aerobic respiration signified by the presence of various respiratory chain subunits, provided the metabolic advantage leading to mitochondrial selection and subsequent evolution (Anderson et al., 2002; Dolezal et al., 2006; Szklarczyk and Huynen, 2010). Evolution of mitochondria itself is not only incremental, having occurred over millions of years, but also dependent on differential symbiotic relationships and environmental conditions, as illustrated by a wide array of mitochondrial genome sizes of different organisms (Gray et al., 1999; Gray, 2011). Moreover, the modern mitochondrial proteome is a result of a series of processes. These processes consist of the loss of ancestral mitochondrial genes, the transfer and integration of mitochondrial genes to the nucleus, and also the evolution of nuclear encoded sequences, which are then imported to the mitochondria and contribute to the majority of its proteome (Anderson et al., 2002). About $99 \%$ of the mitochondrial proteome consists of nuclear encoded proteins; with the remaining $1 \%$ being products of the mitochondrial genome 
(Chacinska et al., 2009). The yeast mitochondrial genome encodes mainly large multitransmembrane hydrophobic subunits of the respiratory chain such as Cox1 of the cytochrome $c$ oxidase (Fontanesi et al., 2006).

Nuclear encoded proteins, which are synthesized on cytosolic ribosomes, are imported by the mitochondrial import machinery, consisting of the necessary complexes, TOM, SAM, TIM23, TIM22, and MIA (Neupert and Herrmann, 2007; Chacinska et al., 2009; van der Laan et al., 2010). Translocases of the outer and inner membranes, TOM and TIM respectively, have no bacterial orthologs, having evolved from the host nuclear genes during endosymbiosis (Kalrberg et al., 2000; Dolezal et al., 2006). Most of the components of the transport machinery formed de novo from nuclear encoded genes with some minor exceptions (Anderson et al., 2002; Dolezal et al., 2006). The outer membrane protein Sam50 is related to the bacterial outer membrane protein BamA (Omp85), whereas homologs of TIM proteins (Tim23 and Tim44) can be found in $\alpha$-proteobacteria (Karlberg et al., 2000; Paschen et al., 2003). Furthermore, Tom7, Tom22, and Tom40 of the TOM complex are suggested to have an endosymbiotic origin (Maćasev et al., 2004).

\subsection{Protein import and biogenesis}

Protein import into mitochondria is dependent on a mitochondrial targeting signal and the mitochondrial import machinery, which ensures the correct localization of the protein within the highly compartmentalized mitochondria. Proteins can be targeted to the mitochondria by containing either an $\mathrm{N}$-terminal cleavable presequence, or alternatively by internal signals which span various segments of the protein sequence (Pfanner and Geissler, 2001; Neupert and Herrmann, 2007). Import into various mitochondrial compartments is dependent on the intrinsic characteristics of the protein: being either a precursor with presequence, a multi-spanning membrane hydrophobic precursor, or a $\beta$-barrel precursor (Neupert and Herrmann, 2007; van der Laan et al., 2010). Import contains mainly two components: a sorting/insertion mechanism and a receptor, the TOM complex, which is a common component in all import pathways (Figure 1). The protein translocating channel of the TOM complex, a $\beta$-barrel translocase, is composed of Tom40 (Ahting et al., 2001; Becker et al., 2005). Tom22 acts as the receptor for presequence containing precursors whereas Tom70 acts as a 
receptor for hydrophobic precursors (Muto et al., 2001; Wu and Sha, 2006). However, various proteins have been shown to be dependent on both Tom22 and Tom70 (van der Laan et al., 2010). In most cases, precursor proteins interact with Tom 20 and Tom5 after binding to the receptor: this allows the interaction with Tom40 and the consequent translocation to the inner membrane (Chacinska et al., 2009). In the case of $\beta$-barrel precursor proteins, after binding to the TOM complex, the proteins are passed to the SAM complex, which then mediates the proper insertion into the mitochondrial outer membrane (Wiedemann et al., 2003). Similarly, hydrophobic precursors are passed to the TIM22 complex in the mitochondrial inner membrane, which then allows the proper insertion and maturation of the protein (Rehling et al., 2003; Peixoto et al., 2007). In both cases, SAM and TIM22 mediated import; precursors interact with small TIM chaperones, composed of Tim9 and Tim10 (Curran et al., 2002).

Alternatively, precursors with presequences interact with the TIM23 complex in the mitochondrial inner membrane. The TIM23 complex exists in two distinct forms in equilibrium, the TIM $23^{\mathrm{CORE}}$ and the TIM $23^{\mathrm{SORT}}$ : The TIM $23^{\mathrm{CORE}}$ complex mediates the translocation of precursor proteins to the matrix whereas the TIM $23^{\text {SORT }}$ mediates lateral sorting of inner membrane targeted precursor proteins that contain a stroptransfer signal adjacent to their N-terminal presequence (van der Laan et al., 2010). The TIM $23^{\text {CORE }}$ works in concert with the PAM complex, which is composed of various J-proteins (PAMs) and the mtHsp70 ATPase; the PAM complex utilizes ATP hydrolysis to translocate precursor proteins to the matrix (Liu et al., 2003). In parallel, the TIM $23^{\text {SORT }}$ complex in association with the respiratory chain, laterally releases membrane-spanning proteins to the inner membrane (Glick et al., 1992; Chacinska et al., 2009). Both TIM23 complexes are dependent on the presence of a membrane potential across the inner membrane (Chacinska et al., 2009; van der Laan et al., 2010). In the absence of a membrane potential, import of precursor proteins is halted at the TOM complex (Frazier et al., 2003). Finally, the mitochondrial presequence protease (MPP), cleaves the N-terminal presequence after proper translocation or lateral release by the Tim23 complex (Yang et al., 1988). 


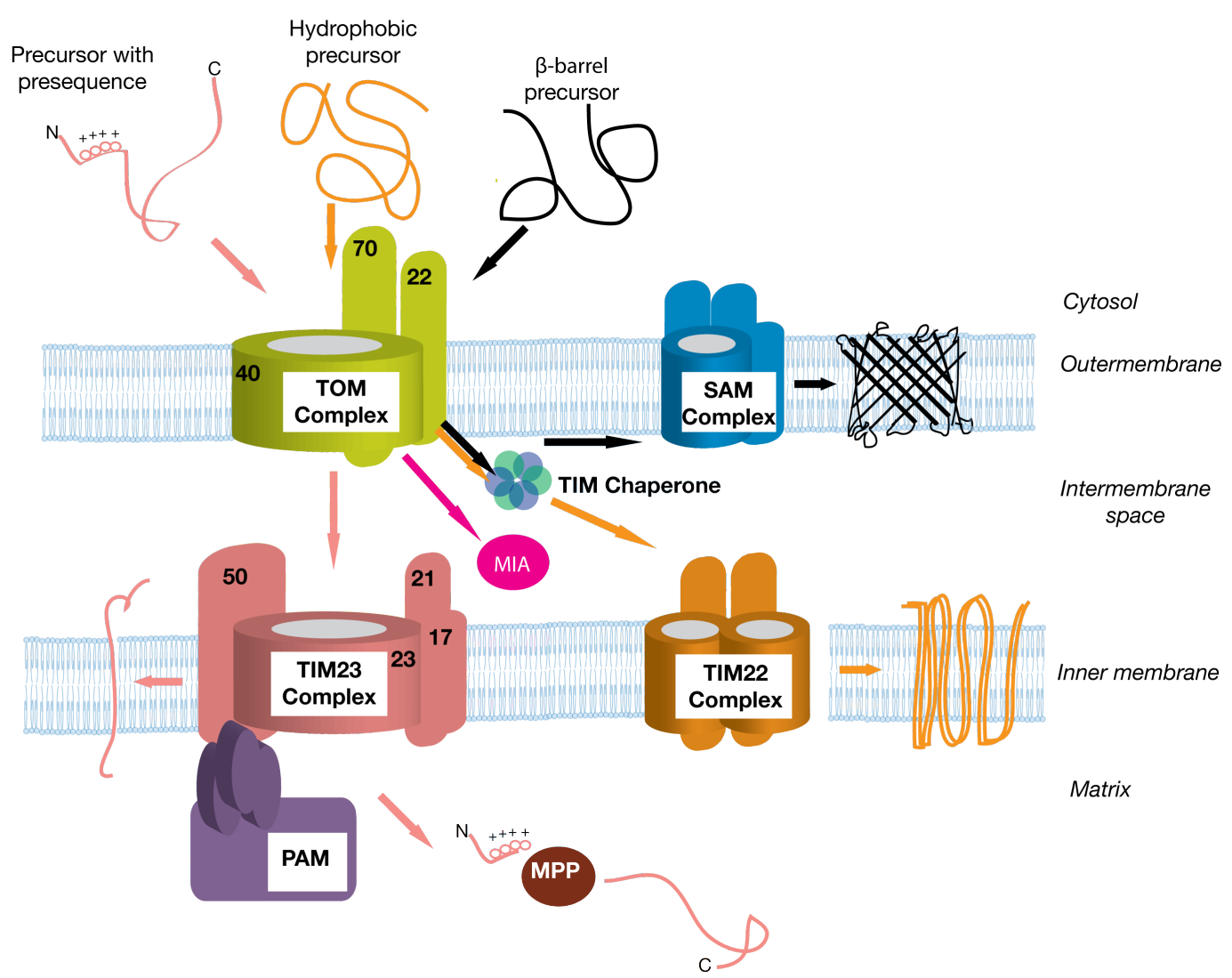

Figure 1. Overview of various mitochondrial import pathways. The TOM complex is the initial and central component in protein import pathways. Figure adapted from van der Laan et al. (2010)

Additionally, many IMS proteins generally lack N-terminal signals and are sorted to the IMS. Their import rather depends on internal cysteine motifs and the mitochondrial disulfide pathway, MIA pathway; this pathway has been found essential for various IMS proteins (Herrmann and Riemer, 2012). The cysteine motifs interact with the Mia40 receptor complex in the IMS (Milenkovic et al., 2007; Sideris and Tokatlidis, 2007). Mia40 is an oxidoreductase, which contains a redox-active cysteine pair residues directly involved in the oxidative folding of proteins (Banci et al., 2009). Furthermore, Mia40 is maintained in its oxidative state by the sulfihydryl oxidase, Erv1, which in turn is reoxidized by cytochrome $c$ (Farrell et al., 2005; Bihlmaier et al., 2007). Oxidative protein folding by disulfide bond formation is thought to drive translocation and targeting to the mitochondrial IMS (Banci et al., 2009). 


\subsection{Oxidative phosphorylation}

Mitochondria are referred to as the powerhouse of the cell due to their central role in oxidative phosphorylation. This process, which is mediated by the respiratory chain, involves the coupled oxidation of $\mathrm{NADH}$ and $\mathrm{FADH}_{2}$ by electron transport and subsequent ATP synthesis by the $\mathrm{F}_{1} \mathrm{~F}_{\mathrm{O}} \mathrm{ATPase}$ in the inner membrane (Figure 2). The respiratory chain is composed of five large multisubunit protein complexes: NADH dehydrogenase (complex I), succinate dehydrogenase (complex II), cytochrome $c$ reductase/ cytochrome $\mathrm{bc}_{1}$ complex (complex III), cytochrome $c$ oxidase (complex IV), and the $\mathrm{F}_{1} \mathrm{~F}_{\mathrm{O}} \mathrm{ATPase}$ (complex V). In brief, oxidative phosphorylation follows electron transport within the respiratory chain, which causes $\mathrm{H}^{+}$translocation from the matrix to the IMS by complexes I, III and IV (Boyer, 1997). This forms a proton gradient across the inner membrane. The $\mathrm{F}_{1} \mathrm{~F}_{\mathrm{O}} \mathrm{ATPase}$ then uses the proton motive force (the sum of the chemical gradient of $\mathrm{H}^{+}$ions and the membrane potential) to produce ATP from ADP and inorganic phosphate (Boyer, 1997). One should note that the yeast Saccharomyces cerevisiae (S.cerevisiae), unlike most eukaryotes, does not contain a membrane embedded complex I, but rather NADH dehydrogenases associated with the inner membrane (Luttik et al., 1998).

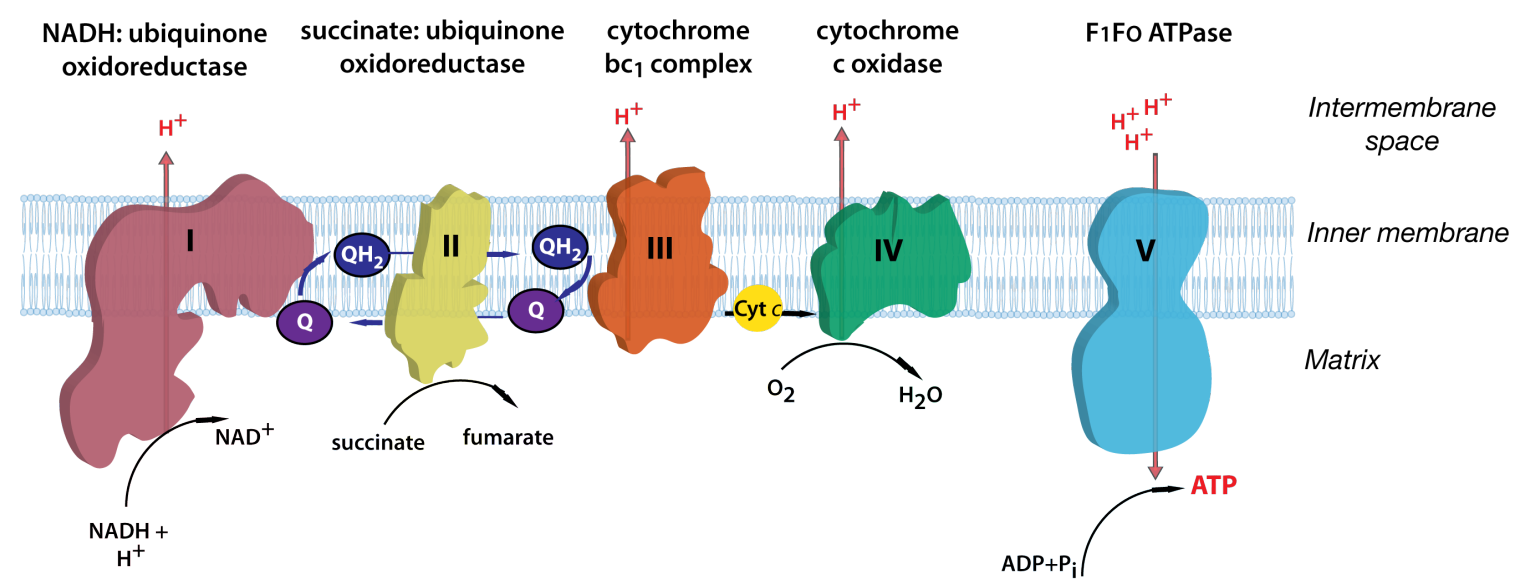

Figure 2. Overview of mitochondrial respiratory chain and oxidative phosphorylation.

\subsection{Respiratory Supercomplex Formation}

The molecular organization of the respiratory chain within the mitochondrial inner membrane has been addressed by two models: 1) the "liquid-state" model which postulates that respiratory chain complexes are arranged randomly and thus diffuse latterly and freely in the membrane and 2) the "solid-state" model which postulates that respiratory chain complexes associate in an ordered manner to form larger 
complexes, or supercomplexes (Vonck and Schäfer, 2009). Evidence for the latter model has been firstly shown in bacteria and then in mitochondria from yeast and higher eukaryotes (Stroh et al., 2004; Stuart, 2008). In S.cerevisiae, four large supercomplexes have been observed by BN-PAGE after solubilization of mitochondria in the mild nonionic detergent, digitonin (Figure 3). These complexes are comprised of a dimeric $\mathrm{F}_{1} \mathrm{~F}_{\mathrm{O}} \mathrm{ATPase}\left(\mathrm{V}_{2}\right)$, a dimeric complex III (III $)$, and a dimeric complex III associated with one or two moieties of complex IV ( $\mathrm{III}_{2} \mathrm{IV}$ and $\left.\mathrm{III}_{2} \mathrm{IV}_{2}\right)$ (Stuart, 2008).

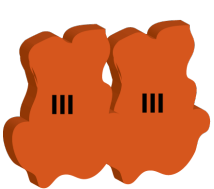

$\mathrm{III}_{2}$

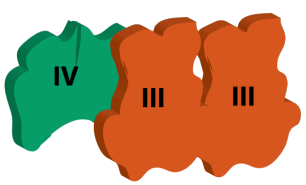

$\mathrm{III}_{2} / \mathrm{IV}$

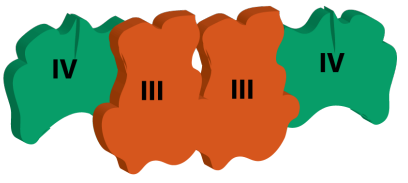

$\mathrm{III}_{2} / \mathrm{IV}_{2}$

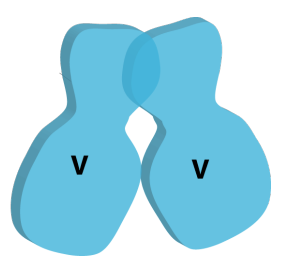

F1Fo ATPase dimer

Figure 3. Structural organization of respiratory chain supercomplexes in yeast. Large supercomplexes have been observed by BN-PAGE: a dimeric $\mathrm{F}_{1} \mathrm{~F}_{\mathrm{O}} \mathrm{ATPase}\left(\mathrm{V}_{2}\right)$, a dimeric complex III ( $\left.\mathrm{III}_{2}\right)$, and a dimeric complex III associated with one or two moieties of complex $\mathrm{IV}\left(\mathrm{III}_{2} \mathrm{IV}\right.$ and $\left.\mathrm{III}_{2} \mathrm{IV}_{2}\right)$.

In contrast, the organization of respiratory chain complexes appeared to be distinct in mammals. Supercomplexes in mammals may include complex I as well as varying copies of complex IV. Using Blue native PAGE (BN-PAGE) after digitonin solubilization, five supercomplex species were observed: a dimeric $F_{1} F_{O} A T P a s e\left(V_{2}\right)$, a dimeric complex III with a monomeric complex $\mathrm{I}\left(\mathrm{III}_{2} \mathrm{I}_{1}\right)$, and a dimeric complex III, with a monomeric complex I, associated with 1-3 complex IV monomers: $\mathrm{III}_{2} \mathrm{I}_{1} \mathrm{IV}_{1}$, $\mathrm{III}_{2} \mathrm{I}_{1} \mathrm{IV}_{2}$, or $\mathrm{III}_{2} \mathrm{I}_{1} \mathrm{IV}_{3}$ (Schägger and Pfieffer, 2000; Althoff et al., 2011). Moreover, under different solubilization conditions such as with TritonX-100, a supercomplex containing dimeric complex III, a single complex I, and four copies of complex IV $\left(\mathrm{III}_{2} \mathrm{I}_{1} \mathrm{IV}_{4}\right)$ were observed (Vonck and Schäfer, 2009). Supercomplexes containing at least a single complex I, complex III, and a dimeric IV have been referred to as "respirasomes", which are structural units that can autonomously carry out respiration (Schägger and Pfieffer, 2000). Furthermore, supercomplexes have been observed in various other species, albeit different configurations, such as plants, which contain three major complexes: $\mathrm{V}_{2}, \mathrm{III}_{2} \mathrm{I}_{1}$ and $\mathrm{III}_{2} \mathrm{I}_{2}$ (Eubel et al., 2003). 


\section{5 $F_{1} F_{0} A T P a s e:$ from structure to function}

The multisubunit $\mathrm{F}_{1} \mathrm{~F}_{\mathrm{O}} \mathrm{ATPase}$ is ubiquitous to all living organisms and has been found in mitochondrial, bacterial plasma membranes and thylakoid membranes in chloroplasts (Walker et al., 1991; Collinson et al., 1994; Boyer, 1997; Seelert and Dencher, 2011). The yeast mitochondrial $\mathrm{F}_{1} \mathrm{~F}_{\mathrm{O}}$-ATPsynthase is composed of at least 17 subunits to form a soluble globular catalytic part, the $\mathrm{F}_{1}$, and a membrane embedded part, the $\mathrm{F}_{\mathrm{O}}$ (Arnold et al., 1998). In addition, various proteins such as Stflp, Stf2p, and Inh1p (IF1 in humans) associate with the $F_{1} F_{O} A T P a s e$ for various regulatory/inhibitory functions (Walker, 1994; Arnold et al., 1998; Hong and Pederson, 2002). Figure 4 summarizes the subunit composition and nomenclature in Escherichia coli (E. coli), the yeast S. cerevisiae, and Homo sapiens (H.sapiens). The $F_{O}$ termed "O" due to sensitivity to the inhibitor oligomycin (Racker, 1963). The enzyme is a multimeric complex of dual genomic origin, with yeast subunits Atp6, Atp8, and Atp9 encoded on the mitochondrial genome and the remaining components being nuclear encoded (Walker et al., 1991, 1995; Arnold et al., 1998). A combination of structural and biochemical data has shone light on the shape of the complex and its enzymatic mechanism (Seelert and Dencher, 2011). The $\mathrm{F}_{1} \mathrm{~F}_{\mathrm{O}} \mathrm{ATPase}$ has been described as a rotary motor, in which proton translocation within the $\mathrm{F}_{\mathrm{O}}$ portion drives the intramolecular rotation of hydrophobic rotor subunits (subunit c oligomers) and eventually movement of a central stalk with the catalytic $F_{1}$ head; this is in concert with nucleotide binding, form the basic of ATP synthesis (Stock et al., 1999). 


\begin{tabular}{l|ll|l|l}
\hline Bacteria & \multicolumn{3}{|c|}{ Mitochondria } & $\begin{array}{l}\text { Domain } \\
\text { Association }\end{array}$ \\
\hline & \multicolumn{2}{|c|}{ S.cerevisiae } & H.sapiens & \\
\hline$\alpha$ & $\alpha$ & & $\alpha$ & \\
$\beta$ & $\beta$ & & $\beta$ & \\
$\gamma$ & $\gamma$ & & $\gamma$ & $\mathrm{F}_{1}$ \\
$\varepsilon$ & $\delta$ & & $\delta$ & \\
- & $\varepsilon$ & & $\varepsilon$ & \\
$\delta$ & OSCP & (Atp5) & OSCP & \\
\hline$a$ & 6 & (Atp6) & $a$ & \\
- & 8 & (Atp8) & A6L & \\
$c$ & 9 & (Atp9) & $c$ & \\
$b$ & 4 & (Atp4) & $b$ & $\mathrm{~F}_{0}$ \\
- & $d$ & & $d$ & \\
- & $h$ & & $\mathrm{~F} 6$ & \\
- & $f$ & & $f$ & \\
- & $e$ & (Atp21) & $e$ & \\
- & $g$ & (Atp20) & $g$ & associated \\
- & $i$ & (Atp18) & - & \\
\hline- & $k$ & (Atp19) & - & \\
\hline- & Inh1 & & IF1 & \\
- & Stf1 & & - & \\
- & Stf2 & & - & \\
\hline
\end{tabular}

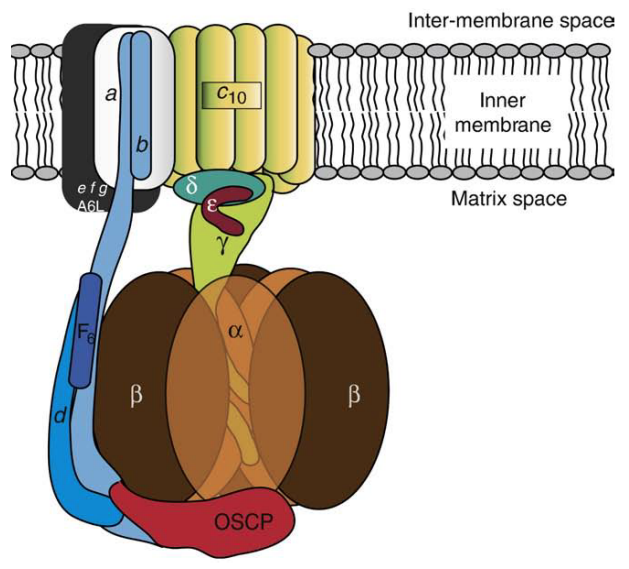

Figure 4. Nomenclature and organization of $F_{1} F_{0} A T P a s e$ subunits in yeast and H.sapiens. (adapted from Kucharczyk et al., 2009)

\subsection{High ordered organization of the $F_{1} F_{0} A T P a s e:$ from dimers to oligomers}

Early electron micrographs revealed that the $\mathrm{F}_{1} \mathrm{~F}_{\mathrm{O}} \mathrm{ATPases}$ are found adjacent to one another (Burton, 1979). Consequently, deep-etch freeze electron microscopy of mitochondria of Paramecium multimicronucleatum, revealed an arrangement of $\mathrm{F}_{1} \mathrm{~F}_{\mathrm{O}} \mathrm{ATPase}$ into double rows of particles (Allen et al., 1989). These initial observations, followed by biochemical isolations with mild detergents and results based on cryo-electrotomographies indicated that the $F_{1} F_{O} A T P a s e$ forms ribbons composed of homodimers at the mitochondrial cristae membranes (Schägger and Pfieffer, 2000; Strauss et al., 2008; Davies et al, 2011). The $\mathrm{F}_{1} \mathrm{~F}_{\mathrm{O}} \mathrm{ATP}$ ase in its dimeric form, has been shown in a wide range of organisms such as mammals, plant species, Drosophila, and various yeast such as P. anserina, N. crassa, and Y. lipolytica (Straus et al., 2008; Davies et al., 2011). Also, higher oligomeric states of the $\mathrm{F}_{1} \mathrm{~F}_{\mathrm{O}} \mathrm{ATPase}$ homodimers have been found (Eubel et al., 2003; Krause et al., 2005; Thomas et al., 2008; De los Rios Castillo et al., 2011). Moreover, studies using atomic force microscopy revealed the $\mathrm{F}_{1} \mathrm{~F}_{\mathrm{O}} \mathrm{ATPase}$ dimers in their native environment (Buzhynskyy et al., 2007). Even though there is significant evidence of the dimeric form, there is yet to be a consensus on the structural nature of homodimeric interactions. Single particle 
cryoelectron microscopy studies indicate that contact sites between the two monomers are located between the two $F_{O}$ membrane portions, whereas other studies revealed an involvement of the $F_{1}$ regions (Seelert and Dencher, 2011). Furthermore, the angles between the two monomers appears to vary dependent on the organism in question: the angle varied between $45^{\circ}$ and $70^{\circ}$ in yeast and Polytomella, while it was found to $40^{\circ}$ for bovine dimers (Seelert et al., 2009).

\subsection{Functional roles of the $F_{1} F_{0} A T P a s e$ dimers}

In combining clear native PAGE (CN-PAGE) together with an in-gel activity $\mathrm{F}_{1} \mathrm{~F}_{\mathrm{O}} \mathrm{ATPase}$ assay, it was shown that monomers, dimers, or oligomers of the $\mathrm{F}_{1} \mathrm{~F}_{\mathrm{O}} \mathrm{ATPase}$ have no significant differences in ATP hydrolysis thus ruling out a role of oligomerization in activity (Krause et al., 2005). This led to the proposal of other functions with respect to $\mathrm{F}_{1} \mathrm{~F}_{\mathrm{O}} \mathrm{ATPase}$ localization at cristae membranes. First, the "quinary structure" model proposes that organizing metabolic enzymes into stoichiometric complexes increases the efficiency of substrate channeling (the direct transfer of metabolic intermediates to subsequent processing steps) (Srere, 1999). Thus together with the associations of other respiratory chain supercomplexes, a "respirasome" is formed, which allow easier electron transport between complexes. Second, a series of $\mathrm{F}_{1} \mathrm{~F}_{\mathrm{O}} \mathrm{ATPase}$ dimers allows the formation of a strong local curvature and thus a local proton trap at the cristae apex (Strauss et al., 2008). The stringent ribbon arrangement at the cristae membrane tips generates a local proton gradient in the adjacent cristae space, thus optimizing the $\Delta \mathrm{pH}$ required for the proton motive force used for ATP synthesis (Buzhynskyy et al., 2007; Strauss et al., 2008; Davies et al., 2011). Moreover, oligomerization of the $\mathrm{F}_{1} \mathrm{~F}_{\mathrm{O}} \mathrm{ATPase}$ in mammals has been considered to serve a regulatory role to reduce ATP synthesis in conditions of low electron transfer activity and reduced membrane potential (Bornhövd et al., 2006). In these instances, oligomerization is thought to be a first-order regulation step, which allows the inhibitor protein IF1 to consequentially bind and reduce $\mathrm{F}_{1} \mathrm{~F}_{\mathrm{O}} \mathrm{ATPase}$ catalytic activity (Wittig and Schägger, 2009).

From a structural point of view, dimerization stabilizes the holo-enzyme structure by connecting the two-stator parts in the dimer (Wittig and Schägger, 2009). Oligomerization compensates torques generated on the stators $\left(\mathrm{F}_{\mathrm{O}}\right)$ allowing only rotor 
$\left(F_{1}\right)$ movement, thus blocking energy dissipation, which may result from holo-enzyme rotation around its own axis in the absence of an adjacent monomer or holo-enzyme (Buzhynskyy et al., 2007).

\section{8 $F_{1} F_{0} A T P a s e$ dimerization factors}

In S.cerevisiae, various proteins have been described and characterized to be dimerization factors of the $\mathrm{F}_{1} \mathrm{~F}_{\mathrm{O}} \mathrm{ATPase}$. The current model of $\mathrm{F}_{1} \mathrm{~F}_{\mathrm{O}} \mathrm{ATPa}$ ( dimerization (Figure 5) entails a step-wise assembly in which dimerization factors associate to the membrane bound $\mathrm{F}_{\mathrm{O}}$ (Wagner et al., 2010). Assembly is mediated by sequential binding of the various subunits to form the final dimeric form. Assembly begins with the association of subunit i (Atp18), followed by subunits g (Atp20) and e (Atp21) association to form a primed monomer. Two primed monomers associate to give rise to a primed dimer (not shown). Final association of the dimerization factor $\mathrm{k}$ (Atp19) gives rise to the mature $\mathrm{F}_{1} \mathrm{~F}_{\mathrm{O}} \mathrm{ATPase}$ dimer.

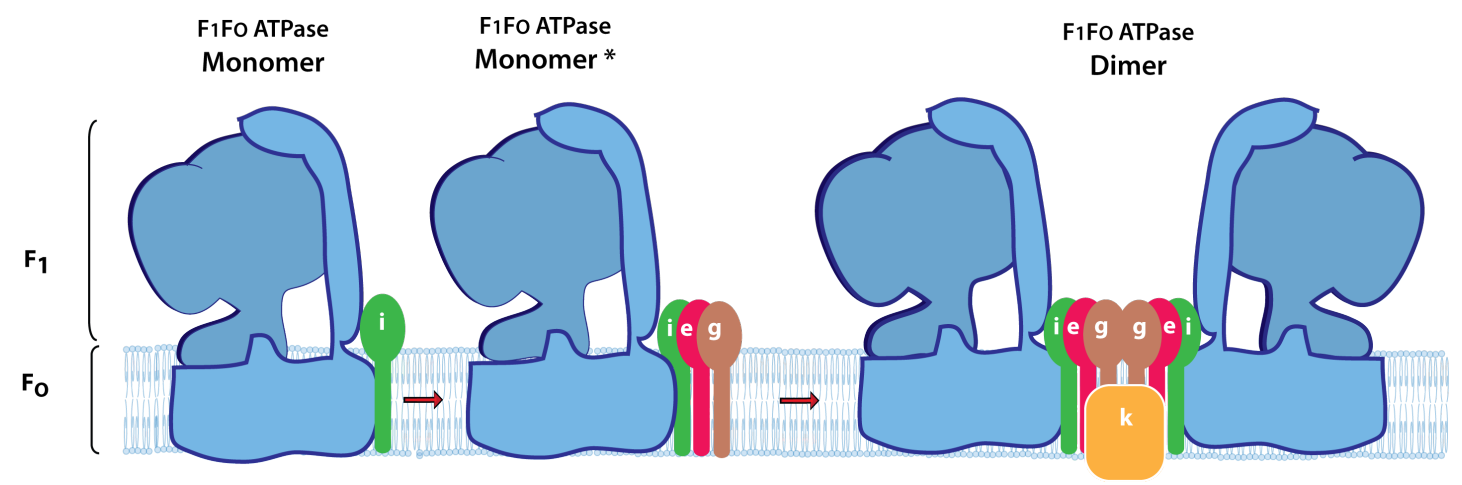

Figure 5. Stepwise assembly of the $\mathbf{F}_{1} \mathbf{F}_{\mathbf{O}} \mathbf{A T P a s e}$ dimer. Sequential association of subunit $g$ (Atp20) and subunit e (Atp21) leads to the formation of a primer monomer. Association of subunit i (Atp18) facilitates the incorporation of Atp20 and Atp21. The association of two primed monomers forms a mature dimer which stably associates with Atp19. Figure adapted from Wagner et al. (2010). * indicates primed monomer.

The described dimerization factors are not essential for $\mathrm{F}_{1} \mathrm{~F}_{\mathrm{O}} \mathrm{ATPase}$ activity, exemplified by unaffected growth of deletion mutants on non-fermentable media (Arnold et al., 1998; Paumard et al., 2002a; Soubannier et al., 2002). Characteristically, these dimerization factors are of small molecular size and contain a single transmembrane domain, which allows them to exert their interaction with the membrane embedded $\mathrm{F}_{\mathrm{O}}$ domain (Arnold et al., 1997, 1998; Soubannier et al., 2002; 
Paumard et al., 2002a; Wagner et al., 2009, 2010). Atp21 contains a C-terminal coiledcoil domain, which has been demonstrated to stabilize the $F_{1} F_{O} A T P a s e$ dimeric form (Everard-Gigot et al., 2005). Moreover, Atp20 and Atp21 both contain a GxxxG motif in their transmembrane domains (Arselin et al., 2003; Bustos and Velours; 2005; Saddar and Stuart, 2005; Bornhövd et al., 2006). This motif is characterized by two glycine residues separated by any three amino acids, and has been previously been demonstrated to stabilize homo- and heterodimers of other membrane proteins such as Glycophorin A, by supporting helix-helix interactions of neighboring membrane proteins (Russ and Engelman, 2000). To this end, a combination of crosslinking approaches and site directed mutagenesis studies has demonstrated the role of the $\mathrm{F}_{1} \mathrm{~F}_{\mathrm{O}} \mathrm{ATPase}$ dimerizations motif; any amino acids changes in the GxxxG motifs of either Atp20 or Atp21 has abolished dimerization (Arselin et al., 2003).

Besides the small $\mathrm{F}_{1} \mathrm{~F}_{\mathrm{O}} \mathrm{ATPase}$ dimerization factors described above, the IF1 protein, which inhibits ATP hydrolysis and thus $\mathrm{F}_{1} \mathrm{~F}_{\mathrm{O}} \mathrm{ATPase}$ activity has been accredited with a role in promoting dimerization in mammalian mitochondria, but not in yeast (García et al., 2006; Campanella et al., 2009; Wittig et al., 2010). Moreover, other subunits of the $\mathrm{F}_{1} \mathrm{~F}_{\mathrm{O}} \mathrm{ATPase}$, such as subunit $\mathrm{a}, b, h$, and $\gamma$, have been shown to play a role in dimerization (Soubannier et al., 2002; Fronzes et al., 2003). Subunit a, component of the ATPase proton channel, localizes to the monomer-monomer interface, and has been demonstrated to have an additive effect to subunits Atp20 and Atp21 in dimerization (Steed and Fillingame, 2008; Wittig et al., 2008; Wittig et al., 2010). The peripheral stalk, connecting the $F_{1}$ and $F_{O}$ domains, is composed of subunits $b$ (subunit 4 in yeast) and h. Subunit $b$ and $h$ of two $\mathrm{F}_{1} \mathrm{~F}_{\mathrm{O}} \mathrm{ATPase}$ monomers have been shown to be in close proximity to each in the dimer and in proximity to subunits Atp18, Atp19, Atp20 and Atp21 (Spannagel et al., 1998; Soubannier et al., 2002; Velours and Arselin, 2000; Wittig et al., 2008). Indeed, manipulation of subunit $b$ first membrane segment and intermembrane space loops prevented dimerization of the $\mathrm{F}_{1} \mathrm{~F}_{\mathrm{O}} \mathrm{ATPase}$ (Soubannier et al., 2002). On the other hand, the $\mathrm{N}$ and $\mathrm{C}$ termini of subunit $\gamma$, part of the catalytic rotor, have been found to be required for proper monomer assembly and dimerization (Dian et al., 2008).

Whereas dimerization factors have been exclusively characterized in S.cerevisiae, information is still lacking on what mediates dimerization of the 
$\mathrm{F}_{1} \mathrm{~F}_{\mathrm{O}} \mathrm{ATPase}$ in higher eukaryotes. Atp20 has a significant homolog (subunit $\mathrm{g}$ ) in mammals and has been described to be part of the holo-enzyme complex, however any role in relation to $\mathrm{F}_{1} \mathrm{~F}_{\mathrm{O}} \mathrm{ATPase}$ dimerization has not been described.

\section{9 $F_{1} F_{0} A T P a s e$ dimerization and maintenance of cristae morphology}

$\mathrm{F}_{1} \mathrm{~F}_{\mathrm{O}} \mathrm{ATPase}$ dimers and oligomers, which align across cristae membrane tips, are directly involved in the maintenance and biogenesis of cristae membranes (Giraurd et al., 2002; Paumard et al., 2002b; Gavin et al., 2004; Rak et al., 2007; Velours et al., 2009). $F_{1} F_{O} A T P a s e$ dimers are conceived to have a role in cristae membrane morphology by inducing a strong local positive curvature of the inner membrane in the direction of the matrix (Dudkina et al., 2006). This, by part, is due the characteristic angle between monomers which has the potential to form an inflexible arc and thereby protrusions of the inner membrane (Paumard et al., 2002b).

Whereas dimerization does not affect ATP synthesis and overall respiratory capacity of mitochondria, the absence of $\mathrm{F}_{1} \mathrm{~F}_{\mathrm{O}} \mathrm{ATPase}$ dimers is directly linked to aberrant mitochondrial inner membrane morphologies (Paumard et al., 2002b; Arselin et al., 2003, 2004; De los Rios Castillo et al., 2004; Minauro-Sanmiguel et al., 2005). The presence of assembled dimers rather than $\mathrm{F}_{1} \mathrm{~F}_{\mathrm{O}} \mathrm{ATPase}$ activity is required for cristae biogenesis (Rak et al., 2007). The absence of the small dimerization factors, Atp20 or Atp21, leads to a disorganized inner mitochondrial membrane, which lack apparent cristae (Figure 6) (Paumard et al., 2002b; Arselin et al., 2003; Arselin et al., 2004). Absence of Atp20 or Atp21 subunits causes enlargement of CJ diameters, promotes cristae branching as well as a decrease in the number of cristae tips (Rabl et al., 2009). Wildtype mitochondria contain poorly defined narrow and diminutive cristae, whereas ATP20 and ATP21 deletion mutants have well defined inner membrane sheets, composed of two or three concentric double leaflet membrane layers which transverse the mitochondria (Paumard et al., 2002b; Arselin et al., 2004). In these mutants, the inner membrane is thus described to have 'onion-like' structures, which are understood to be a result of uncontrolled biogenesis of the inner membrane (Arselin et al., 2004). 

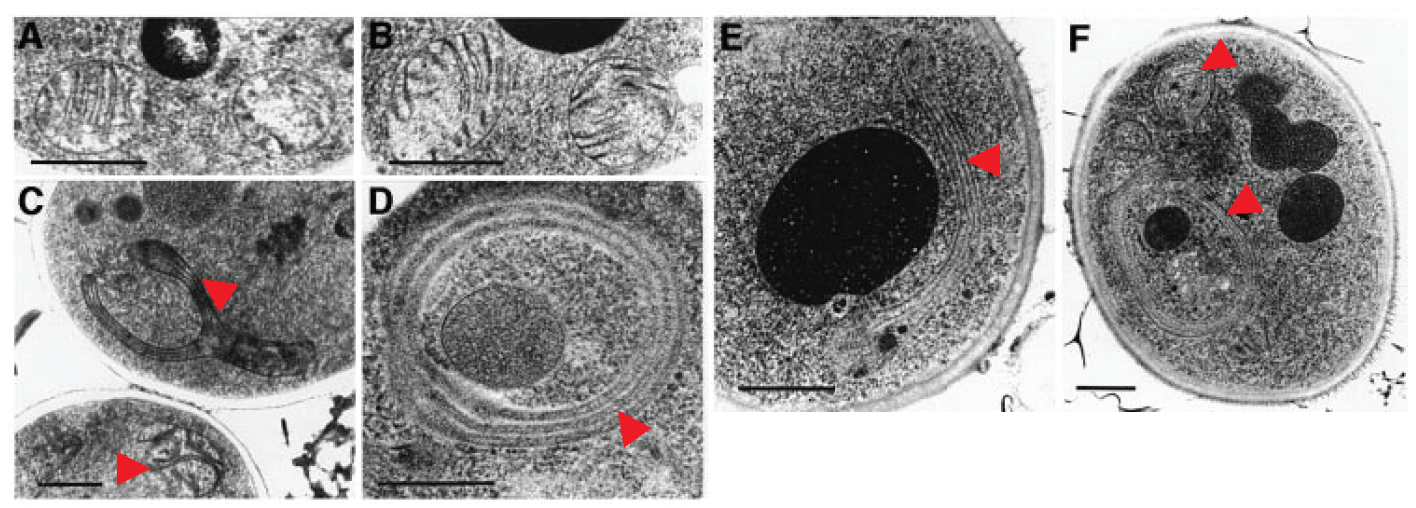

Figure 6. Aberrant mitochondrial morphology in ATP20 and ATP21 deletion mutants. Figure adapted from Paumard et al., 2002. Electron micrographs; Scale bars indicate $0.5 \mathrm{~mm}$.

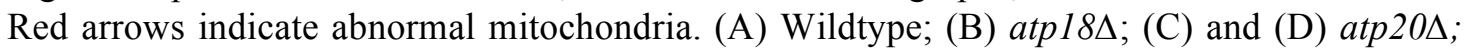
(E) and (F) atp $21 \Delta$.

In addition, crosslinking $\mathrm{F}_{1} \mathrm{~F}_{\mathrm{O}} \mathrm{ATPase}$ dimers via the $b$ subunits, Atp18, or $\gamma$ subunits has been shown to eliminate cristae (Spannagel et al., 1998; Paumard et al., 2002b; Gavin et al., 2004). The absence of subunit $b$ (subunit 4/Atp4), involved in dimerization, also causes, albeit smaller, onion-like structures (Soubannier et al., 2002). Similar small onion-like structures are also observed in rho- mitochondria (Paumard et al., 2002b). Since atp $4 \Delta$ mutants, which lack a fully assembled $\mathrm{F}_{1} \mathrm{~F}_{\mathrm{O}} \mathrm{ATPase}$ and rho $^{-}$mitochondria are respiratory deficient, smaller onion-like structures are believed to be formed due to the lack of sufficient energy for the biogenesis and extension of the inner membrane observed in ATP20 and ATP21 deletion mutants (Paumard et al., 2002b). Nonetheless, the presence of a functional $\mathrm{F}_{1} \mathrm{~F}_{\mathrm{O}} \mathrm{ATPase}$ is required for cristae biogenesis, exemplified by analysis of Atp1, Atp2, and Atp12 mutants, which completely lack cristae (Paumard et al., 2002b). It is thus deduced that the lack of Atp20 or Atp21 in combination with an assembled functional $\mathrm{F}_{1} \mathrm{~F}_{\mathrm{O}} \mathrm{ATPase}$ leads to the 'onion-like' phenotype observed in the mutants (Paumard et al., 2002b; Velours et al., 2009).

\subsection{The inner membrane and cristae membrane architecture}

Besides components of $\mathrm{F}_{1} \mathrm{~F}_{\mathrm{O}} \mathrm{ATPase}$, various other inner membrane proteins play a role in inner membrane and cristae organization. These proteins include Mitofilin (and its yeast homolog Fcj1) and CHCHD3 (John et al., 2005; Rabl et al., 2009; Darshi et 
al., 2011). Moreover, certain proteins involved in apoptosis (OPA1/Mgm1) and mitochondrial fusion and fission play a part in determining proper cristae morphology.

The highly abundant mitochondrial protein Mitofilin is expressed as two splice variants with protein products sized 88 and $90 \mathrm{kDa}$ respectively (Odgren et al., 1996; Gieffers et al., 1997). Mitofilin is characterized by a cleavable N-terminal targeting sequence, a membrane-anchoring domain, and central coiled coil domain (Odgren et al., 1996; John et al., 2005). It is anchored to the inner membrane in which the majority of its surface is exposed to the IMS and is enriched in the narrow interface located between the inner and outer membranes (Gieffers et al., 1997; John et al., 2005). A role for Mitofilin in cristae biogenesis has been deduced when its protein levels were down-regulated by RNA interference (RNAi) in HeLa cultured cells, in which the mitochondrial inner membrane was disorganized into a membranous labyrinth composed of tightly packed stacks of membrane sheets (John et al., 2005). Moreover, the inner membranes, which had an increased inner membrane to outer membrane ratio, were devoid of cristae and cristae junctions, highlighting a role for Mitofilin in cristae junction formation (John et al., 2005).

The role of Mitofilin in inner membrane biogenesis appears to be conserved in other species. Mutation of the two C.elegans homologs, IMMT-1 and IMMT-2, also leads to stacked inner membrane networks and to a reduced number of cristae junctions (Mun et al., 2010). Interestingly, the Mitofilin C. elegans homologs, IMMT1 and IMMT-2 are described to behave as large complexes associated with the $\mathrm{F}_{1} \mathrm{~F}_{\mathrm{O}} \mathrm{ATPase}$ in CN-PAGE (Mun et al., 2010). The yeast homolog, Fcj1 (formation of cristae junction 1) is also involved in cristae biogenesis (Rabl et al., 2009). Fcj1, like Mitofilin, is enriched at cristae junctions at the mitochondrial inner membrane and is primarily exposed to the IMS. Similarly, yeast mutants that lack Fcj1, are devoid of cristae junctions and exhibit aberrant mitochondrial morphology exemplified by parallel concentric stacks of inner membrane. A direct role for Fcj1 in cristae biogenesis has been shown by overexpression of $F C J 1$, which leads to an increase in cristae junction diameters, as well as increased cristae junction formation and branching (Rabl et al., 2009). 
In addition, there is an antagonism between Fcj1 and $F_{1} F_{O} A T P a s e$ subunits Atp20 and Atp21 in controlling local inner membrane curvature and cristae formation. Fcj1 does not affect $F_{1} F_{O} A T P a s e$ monomers or dimers but rather impairs the formation of higher oligomers (Rabl et al., 2009). Whereas deletion of FCJ1 does not affect steady state protein levels of Atp20 or Atp21, nor does the deletion of ATP20 or ATP21 affect Fcj1 levels, a double deletion of either FCJ1/ATP20 or FCJ1/ATP21 leads to the formation of concentric (vesicle-like) cristae membranes completely devoid of cristae junctions. In this antagonistic model, the presence of $\mathrm{F}_{1} \mathrm{~F}_{\mathrm{O}} \mathrm{ATPase}$ oligomers and thus dimerization factors Atp20 and Atp21 favors positive membrane curvature and ensuing formation of cristae tips (Rabl et al., 2009). In contrast, Fcj1 destabilizes $\mathrm{F}_{1} \mathrm{~F}_{\mathrm{O}} \mathrm{ATP}$ ase oligomerization, thus allowing cristae junction formation by favoring negative membrane curvature over positive curvature. Thus, differential localization of Fcj1 and Atp20/Atp21 at the mitochondrial inner membrane allows proper cristae junction and cristae tip formations (Rabl et al., 2009).

Immunoprecipitations and a yeast two-hybrid assay indicated that Mitofilin form a homotypic interaction assembling into a large ( $>1 \mathrm{MDa})$ complex residing at the inner membrane/outer membrane interface (John et al., 2005). Also, it was shown to interact with six other proteins: Metaxin 1, Metaxin 2, SAM50, CHCHD6, DnaJC11, and CHCHD3 (Xie et al., 2007). Metaxins 1 and 2 are cytosolic proteins, which associate with the mitochondrial outer membrane and are involved in protein import to the mitochondria (Abdul et al., 2000). SAM50, component of the outer membrane SAM complex is also involved in protein import and biogenesis, whereas DnaJC11 is a member of the $40 \mathrm{kDa}$ heat shock protein family and may be involved in protein import and folding (Xie et al., 2007). The evidence of Mitofilin interaction with the Metaxins, SAM50, and DnaJC11, thus links cristae junction formation with mitochondrial protein import. Moreover, CHCHD6 has been recently described to take part in the regulation of mitochondrial cristae morphology, cell growth, ATP production and oxygen consumption (An et al., 2012).

In addition, CHCHD3 is critical for cristae structure (Darshi et al. 2011). The protein is highly conserved in mammals with no homologs found in fungi or plants. Knockdown of CHCHD3 resulted in fragmented cristae or even their complete loss. Moreover, the average opening diameter of cristae junctions was reduced by $50 \%$ but 
number of cristae junction was not affected, indicating a possible role of CHCHD3 in cristae junction architecture and maintenance rather than formation (Darshi et al., 2011).

All together, Mitofilins (Mitofilin/ Fcj1/ IMMT-1, IMMT-2) may control the formation of cristae junctions by inhibiting excessive growth and expansion of the inner membrane (John et al., 2005, Rabl et al., 2009, Mun et al., 2010). By forming a multimeric complex via homotypic interactions, they may form a filamentous scaffold, which supports the inner membrane and prevents its expansion (John et al., 2005, Xie et al., 2007, Mun et al., 2010). Evidence of Mitofilin interaction with the Metaxins and SAM50, not only links the cristae maintenance machinery with mitochondrial protein import, but also connects the inner membrane with the outer membrane, an interaction which may further support a rigid scaffolding model in maintenance of mitochondrial inner membrane architecture.

The overall shape of the mitochondrial reticulum is dependent on continuous mitochondrial fission and fusion processes, which regulate mitochondrial morphology and number. Mitochondrial fission in yeast is dependent on the evolutionary conserved GTPase Fzo (Mfn in mammals), Ugo1, as well as Mgm1 (OPA1 in mammals) (Okamato and Shaw, 2005). OPA1/Mgm1, a large dynamin related GTPase, is anchored at the cristae junctions and exposed to the IMS (Olichon et al., 2002). Mitochondria with mutated or depleted OPA1/Mgm1 in yeast or HeLa cultured cells have fragmented spherical mitochondria with characteristically simple inner membrane organization, an effect of disrupted mitochondrial fusion (Olichon et al., 2002; Griparic et al., 2004; Mannella 2008). Moreover, depletion of OPA1 leads to the formation of aberrant curved vesicle-like cristae (Olichon et al., 2002). Besides its role in maintenance of the mitochondrial inner membrane, OPA1 has a role in apoptosis. Fragmentation of mitochondria during apoptosis is dependent on the proteolytic processing of OPA1 (Duvezin-Caubet et al., 2006), whereas a loss of OPA1 commits cells to apoptosis in the absence of further stimuli (Olichon et al., 2003). The role of OPA1 in apoptosis is proposed to involve cytochrome $c$ sequestration and compartmentalization within the cristae membrane (Olichon et al., 2003). This may be mediated by OPA1 localization to the inner membrane and its control of cristae junction openings, hence preventing premature release of cytochrome $\mathrm{c}$ in the absence 
of apoptotic stimuli (Frezza et al., 2006). Interestingly, OPA1 function links cristae biogenesis and apoptosis, which includes extensive remodeling of the cristae membrane (Scorrano et al., 2002). This relationship is further exemplified by protein MICS1 that is involved in both maintenance of mitochondrial morphology and cytochrome $c$ release during apoptosis (Oka et al., 2008). MICS1-depleted mitochondria, like OPA1/Mgm1 mutants, had curved vesicle-like cristae (Oka et al., 2008).

Mgm1 has been described to have a chaperone-like function of the $\mathrm{F}_{1} \mathrm{~F}_{\mathrm{O}} \mathrm{ATPase}$ dimerization factor Atp21, in which the loss of Mgm1 inhibits $\mathrm{F}_{1} \mathrm{~F}_{\mathrm{O}} \mathrm{ATPase}$ dimerization (Amutha et al., 2004). This effect on dimerization might explain cristae membrane anomalies of Mgm1/OPA1 mutants (Mannella, 2008). Moreover, OPA1 has been shown to exist in a complex containing Mitofilin and SAM50, further advocating its role in the regulation of cristae morphology (Darshi et al., 2011). This is further exemplified by the observation that depletion of Prohibitins (proteins involved in regulation of OPA1) leads to loss of cristae (Osman et al., 2009; Zick et al., 2009). Overall, OPA1/Mgm1 demonstrates a role in mitochondrial fusion in cristae morphology. In addition, loss of cristae is observed in mutants of Mdm33, a protein involved in inner membrane fission (Messerschmitt et al., 2003; Okamoto and Shaw, 2005).

\subsection{Models of cristae biogenesis}

Evidently, various proteins integrally or peripherally associated with the mitochondrial inner membrane are involved in the biogenesis and maintenance of cristae membrane. The differences in the inner membrane morphologies of different mutants highlight the differences in the various proteins' functions exemplified by their different localizations (Figure 7). $\mathrm{F}_{1} \mathrm{~F}_{\mathrm{O}} \mathrm{ATPase}$ dimers favor positive curvature and are vital for cristae membrane tip formation, whereas Mitofilin/Fcj1 and OPA1 are required for cristae junction maintenance. The processes governing cristae morphology yet appear to be interconnected by dependence of Atp21 on OPA1/Mgm1 (Amutha et al., 2004) and modulation of Mitofilin/Fcj1 or $\mathrm{F}_{1} \mathrm{~F}_{\mathrm{O}} \mathrm{ATPase}$ oligomerization (Rabl et al., 2009). 


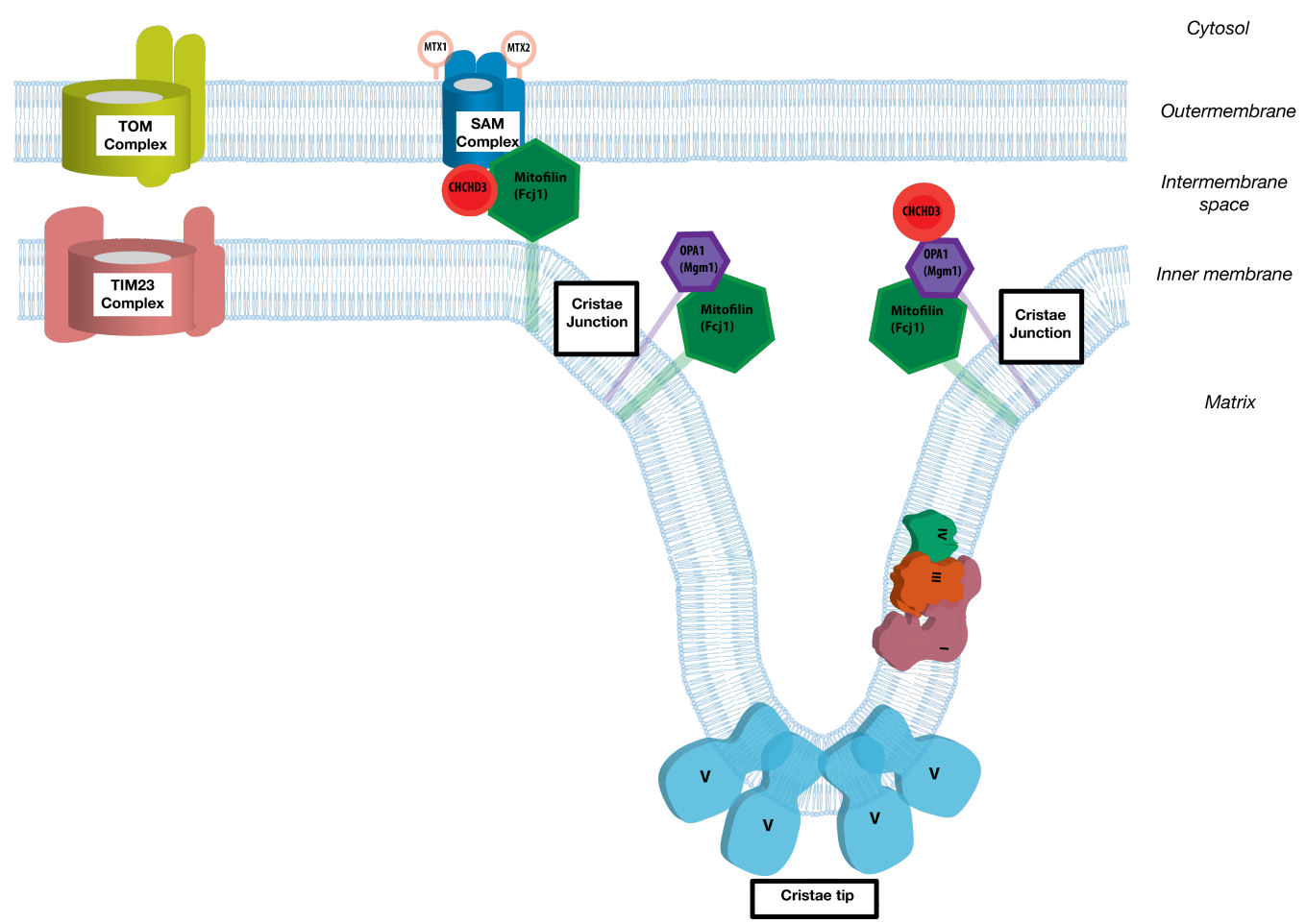

Figure 7. Overview of factors involved in cristae biogenesis and maintenance. The import translocases (TOM and TIM) are found at the IBM, whereas the respiratory chain is found at cristae membranes. The $\mathrm{F}_{1} \mathrm{~F}_{\mathrm{O}} \mathrm{ATPase}$ is located at cristae tips/apex and contributes to cristae shape. Mitofilin and OPA1 are located at cristae openings and control cristae junction width. Mitofilin also interacts with CHCHD3, the SAM complex, and Metaxins.

With respect to cristae, various theoretical models have been proposed to explain cristae biogenesis, illustrated in Figure 8, as reviewed by Zick et al. (2009). Consideration of these models may provide explanations for the various aberrant phenotypes of mitochondrial morphology mutants.

\section{A) Invagination model / Late cristae junction model}

This model postulates that bending energy is sufficient to form spontaneous invaginations of the inner membrane giving rising to cristae membranes provided the continuous biogenesis of the inner membrane and a constant outer membrane surface (Renken et al., 2002). The proposed bending energy can be derived from lipid composition and/or curvatures mediated by large protein complexes such as the $\mathrm{F}_{1} \mathrm{~F}_{\mathrm{O}} \mathrm{ATPase}$ dimer (Paumard et al., 2002a). Consequently, cristae junctions are formed once cristae membrane tips are made. 


\section{B) Balloon model / Early cristae junction model}

Stable ring-like nascent cristae junction structures are formed at the IBM. Cristae are then formed by invagination, in which the nascent cristae junction structures act as an entry point for lipids and proteins (Zick et al., 2009).

C) De novo vesicle germination model

Cristae membranes are formed from mitochondrial matrix vesicles that are formed 'de novo' from single phospholipids particles that later fuse to the IBM (Zick et al., 2009).

D) Cristae fusion/fission model

This model proposes that cristae formation is dynamic, in which cristae membrane vesicles may continuously fuse from one side of the inner membrane to another (fission/fusion). Moreover, the observation that cristae morphology changes under different physiological conditions in a reversible manner supports dynamic rather than static nature of cristae propagation (Mannella et al., 2001).

E) Fusion remnant model

This model proposes that during mitochondrial fusion, cristae junctions initially formed when the inner membrane of two mitochondria fuse together. Cristae membranes are thus remnants of the fusion event. Membrane remodeling is then required to induce curvatures leading to a tubular cristae membrane structure.

\section{F) Hemifusion model}

Similar to the 'fusion remnant' model, cristae membranes are formed in events following mitochondrial fusion. The site, at which two opposing inner membranes fuse, forms a phospholipids vesicle in the matrix, thus also combining the 'de novo vesicle germination model'. Once the vesicle is formed, it fuses to the IBM to form cristae. This model makes the assumption that inner mitochondrial fusion is mediated by hemi-fusion, a membrane fusion intermediate formed when leaflets of two opposing membranes merge while distal remain distant (Chernomordik and Kozlov, 2005). Hemi-fusion allows the formation of the cristae-vesicle. 


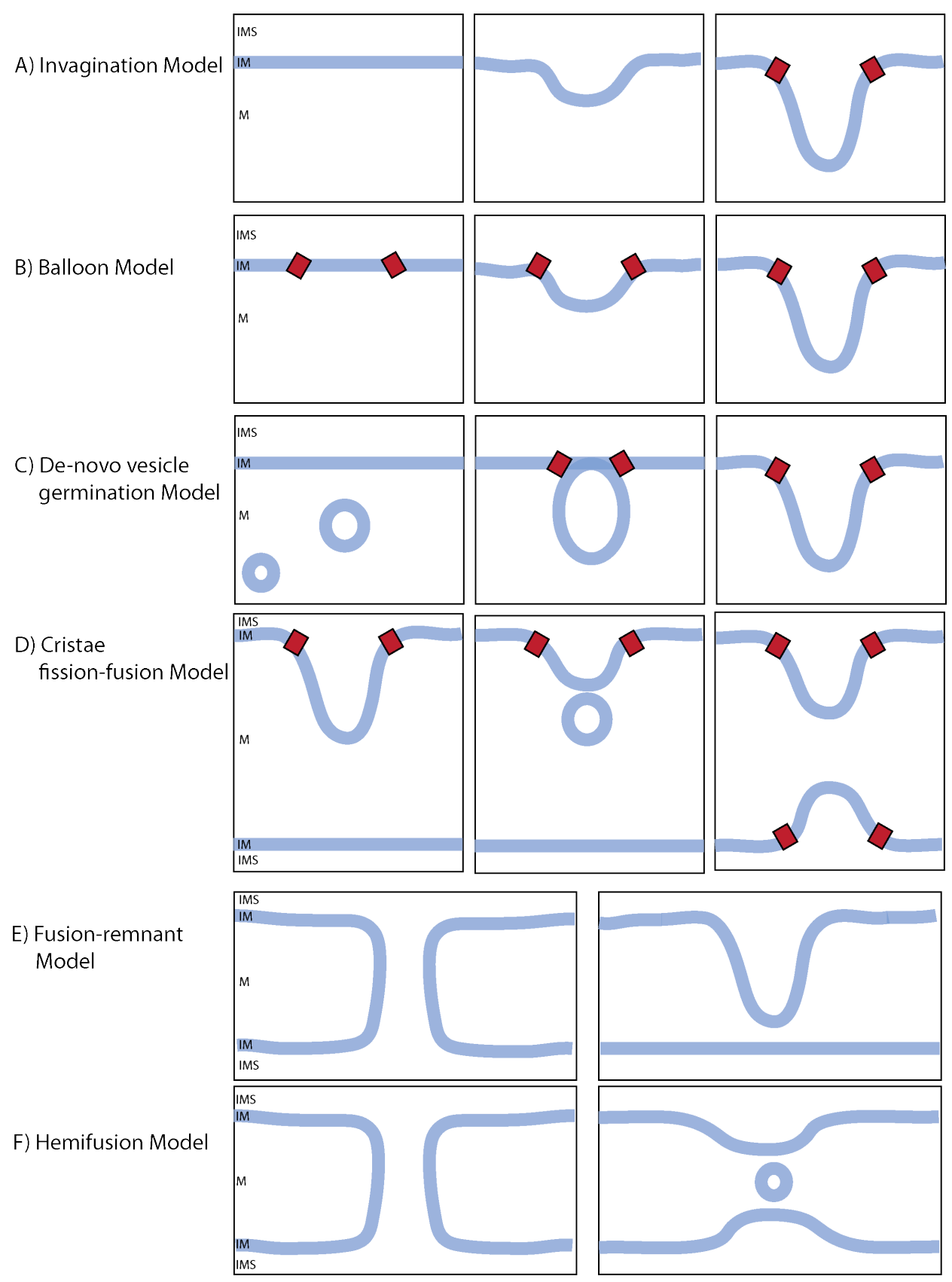

Figure 8. Hypothetical models of cristae biogenesis. Adapted from Zick et al. (2009). IM, inner membrane; $\mathrm{M}$, matrix. 


\subsection{Aims of this study}

Structural subunits of the $\mathrm{F}_{1} \mathrm{~F}_{\mathrm{O}} \mathrm{ATPase}$ are involved in dimerization (Soubannier et al., 2002; Fronzes et al., 2003). In addition, small $F_{O}$ associated yeast proteins (Atp18, Atp19, Atp20, and Atp21) mediate dimerization in yeast (Arnold et al., 1997, 1998; Soubannier et al., 2002; Paumard et al., 2002a; Wagner et al., 2009, 2010). However, their presence does not seem to be conserved in higher eukaryotes. To this end, the aim of this study is to identify novel $\mathrm{F}_{1} \mathrm{~F}_{\mathrm{O}} \mathrm{ATPase}$ dimerization factors in higher eukaryotes. For this purpose, an in silico based search will be used. The bioinformatics approach will be based on searching yeast open reading frames (ORFs) with human homologs. The search will also take into account the various characteristics of known dimerization factors. Mitochondria isolated from human cultured cells (HEK29T) cells will be used to analyze putative $\mathrm{F}_{1} \mathrm{~F}_{\mathrm{O}} \mathrm{ATPase}$ dimerization factors. On the other hand, mitochondria isolated from S.cerevisiae will be used to analyze the yeast homologs. Once identified, putative factors will be characterized for possible functions in dimerization. As the $\mathrm{F}_{1} \mathrm{~F}_{\mathrm{O}} \mathrm{ATPase}$ dimerization factors are membrane proteins (Wagner et al., 2010), putative dimerization factors will be tested first for their membrane association. A role in dimerization will be assessed by investigating $\mathrm{F}_{1} \mathrm{~F}_{\mathrm{O}} \mathrm{ATPase}$ interaction and by analyzing the various available yeast mutants. In addition, as the absence of the dimerization factors Atp20 and Atp21 lead to various aberrations in cristae morphology (Paumard et al., 2002b; Arselin et al., 2003; Arselin et al., 2004), a role in mitochondrial architecture that putative dimerization factors might have will also be considered. 


\section{MATERIALS AND METHODS}

\subsection{Materials}

\subsubsection{Chemicals}

\begin{tabular}{|c|c|}
\hline Product & Supplier \\
\hline Acetone & AppliChem (Steinheim, Germany) \\
\hline Acetic acid & Roth (Karlsruhe, Germany) \\
\hline Acrylamide/ Bisacrylamide solution & Roth (Karlsruhe, Germany) \\
\hline Agar-Agar & Roth (Karlsruhe, Germany) \\
\hline Adenosine triphosphate (ATP) & Sigma (Steinheim, Germany) \\
\hline Agarose NEEO Ultra-Qualität Roti ${ }^{\circledR}$ garose & Roth (Karlsruhe, Germany) \\
\hline Ammonium acetate & Merck (Darmstadt, Germany) \\
\hline Ammoniumpersulsulfate (APS) & Merck (Darmstadt, Germany) \\
\hline Ampicillin & AppliChem (Steinheim, Germany) \\
\hline Bacto $^{\text {TMPeptone }}$ & BD (Sparks, USA) \\
\hline Bacto $^{\mathrm{TM}}$ Tryptone & BD (Sparks, USA) \\
\hline Bacto $^{\mathrm{TM}}$ Yeast Extract & BD (Sparks, USA) \\
\hline Bis-Tris & Roth (Karlsruhe, Germany) \\
\hline Bovine serum albumin (BSA) & Sigma (Steinheim, Germany) \\
\hline Bradford-Reagent & Bio-rad (Richmond, USA) \\
\hline Bromophenoblue & Merck (Darmstadt, Germany) \\
\hline 6-amino Caproic acid & Sigma (Steinheim, Germany) \\
\hline Coomassie Brilliant Blue G-250 & Serva (Heidelberg, Germany) \\
\hline Coomassie Brilliant Blue R-250 & Serva (Heidelberg, Germany) \\
\hline Complete Supplement Mixture - Uracil & MP Biomedicals (Eschwege, \\
\hline (CSM-URA) & Germany) \\
\hline Creatine Phosphate & Roche (Manheim, Germany) \\
\hline L-Cysteine. $\mathrm{HCl}$ & Merck (Darmstadt, Germany) \\
\hline Cytochrome $c$ (bovine heart) & Sigma (Steinheim, Germany \\
\hline Desthio-Biotin & IBA GmbH (Göttingen, Germany) \\
\hline 3,3' Diaminobenzidine (DAB) & Sigma (Steinheim, Germany \\
\hline Digitonin & Calbiochem (La Jolla, U.S.A) \\
\hline Dimethyl pimelimidate dihydrochloride (DMP) & Sigma (Steinheim, Germany) \\
\hline Dimethylsulfoxide (DMSO) & Applichem (Darmstadt, Germany) \\
\hline Dipotassium hydrogen phosphate & Roth (Karlsruhe, Germany) \\
\hline Dithiothreitol (DTT) & Roth (Karlsruhe, Germany) \\
\hline Dulbecco's Modified Eagle Medium (DMEM) & Gibco (Karlsruhe, Germany) \\
\hline Ethidium bromide & AppliChem \\
\hline Ethylenediaminetetraacetic acid (EDTA) & Roth (Karlsruhe, Germany) \\
\hline Glucose & Roth (Karlsruhe, Germany) \\
\hline Glycerin & Roth (Karlsruhe, Germany) \\
\hline Glycin & Roth (Karlsruhe, Germany) \\
\hline Glycerol & Sigma (Steinheim, Germany) \\
\hline $\begin{array}{l}\text { Heavy isotope labeled }{ }^{13} \mathrm{C}_{6}{ }^{15} \mathrm{~N}_{2} \text {-lysine and } \\
{ }^{13} \mathrm{C}_{6}{ }^{15} \mathrm{~N}_{4} \text {-arginine }\end{array}$ & $\begin{array}{l}\text { Euriso-Top (Saarbrücken, } \\
\text { Germany) }\end{array}$ \\
\hline HEPES & Roth (Karlsruhe, Germany) \\
\hline
\end{tabular}


Herring sperm

Lead(II) nitrate $\mathrm{Pb}\left(\mathrm{NO}_{3}\right)_{2}$

Magnesium acetate

Magnesium chloride $\left(\mathrm{MgCl}_{2}\right)$

Magnesium sulfate $\left(\mathrm{MgSO}_{4}\right)$

ß-Mercaptoethanol

Methanol

Milk

L-Methionine

3-(N-morpholino) propanesulfonic acid (MOPS)

Lithium acetate

Nicotinamide adenine dinucleotide hydrate

(NADH)

4-Nitrotetrazoliumchrolide blue (NBT)

N,N,N',N'-Tetramethylethylene diamine

(TEMED)

Polyethylene glycol 4000 (PEG-4000)

PMSF

Potassium acetate

Potassium chloride

Potassium dihydrogen phosphate

Sodium borat

Sodium azide

Sodium Chloride

Sodium deoxycholate

Sodium-dithionite (Na-dithionite)

Sorbitol

SDS

Tricine

Trifluoroacetic acid (TCA)

Tris(hydroxmethlyl)-aminomethane (Tris),

Triton X-100

Tween-20

Yeast Nitrogen base

L-uracil
Promega (Mannheim, Germany)

Sigma (Steinheim, Germany)

Merck (Darmstadt, Germany)

Merck (Darmstadt, Germany)

Roth (Karlsruhe, Germany)

Sigma (Steinheim, Germany)

Roth, (Karlsruhe, Germany)

Reformhaus (Essen, Germany)

Serva (Heidelberg, Germany)

Sigma (Steinheim, Germany)

Applichem (Darmstadt, Germany)

Roche (Manheim, Germany)

Sigma (Steinheim, Germany)

Roth (Karlsruhe, Germany)

Merck (Darmstadt, Germany)

Roth (Karlsruhe, Germany)

Merck (Darmstadt, Germany)

Roth (Karlsruhe, Germany)

Merck (Darmstadt, Germany)

Sigma (Steinheim, Germany)

Sigma (Steinheim, Germany)

Roth (Karlsruhe, Germany)

Sigma (Steinheim, Germany)

Fluka (Steinheim, Germany)

MP Biomedicals (Illkirch, France)

Roth (Kalrsruhe, Germany)

Roth (Karlsruhe, Germany)

Merck (Darmstadt, Germany)

Roth (Karlsruhe, Germany)

Sigma (Steinheim, Germany)

Roth (Karlsruhe, Germany)

Difco BD (New Jersey, USA)

Sigma (Steinheim, Germany)

\subsubsection{General buffers and solutions}

\begin{tabular}{l|l} 
Buffer & Recipe \\
\hline SDS-PAGE sample buffer & $2 \%$ SDS, 10\% Glycerol, 60 mM Tris pH 6.8, \\
& $1 \%$ B-mercaptoethanol, 0.01\% \\
& Bromophenolblue \\
SDS-PAGE running buffer & $25 \mathrm{mM}$ Tris $\mathrm{pH} 6.8,190 \mathrm{mM}$ Glycine, $1 \%(\mathrm{w} / \mathrm{v})$ \\
& SDS \\
SDS-PAGE resolving gel buffer & $1.87 \mathrm{M}$ Tris $\mathrm{pH} 8.8$ \\
SDS-PAGE stacking gel buffer & $0.8 \mathrm{M}$ Tris $\mathrm{pH} 6.8$ \\
Transfer/Blot Buffer & $20 \mathrm{mM}$ Tris \\
& $150 \mathrm{mM}$ Glycin \\
& $0.02 \%$ SDS
\end{tabular}


3x BN-PAGE gel Buffer

40x BN-PAGE Anode Buffer 10x BN-PAGE Cathode Buffer

10x BN Loading Dye

10X TBS

TBS-T

TE buffer

Standard Import Buffer

SEM Buffer

KPi Buffer

10x PBS Buffer

Gel Staining Solution

Gel Destaining Solution

50x TAE Buffer
$20 \%$ Methanol or Ethanol

$200 \mathrm{mM}$ 6-amino caproic acid

$150 \mathrm{mM}$ Bis-Tris / $\mathrm{HCl} \mathrm{pH} 7.0$

$2 \mathrm{M}$ Bis-Tris / $\mathrm{HCl} \mathrm{pH} 7.0$

$500 \mathrm{mM}$ Tricine $\mathrm{pH} 7.0$

$150 \mathrm{mM}$ Bis-Tris

$0.2 \%$ Coomassie blue G250

$5 \%$ Coomassie blue G250

$500 \mathrm{mM}$ 6-amino caproic acid

$100 \mathrm{mM}$ Bis-Tris $\mathrm{pH} 7.0$

$200 \mathrm{mM}$ Tris- $\mathrm{HCl} \mathrm{pH} 7.5$

$1.25 \mathrm{M} \mathrm{NaCl}$

$1 \mathrm{x}$ TBS

$0.1 \%$ Tween-20

$10 \mathrm{mM}$ Tris-Cl, $\mathrm{pH}$ 7.5. $1 \mathrm{mM}$ EDTA

$3 \%(\mathrm{w} / \mathrm{v})$ fatty acid free BSA

$250 \mathrm{mM}$ sucrose

$80 \mathrm{mM} \mathrm{KCl}$

$5 \mathrm{mM} \mathrm{MgCl}_{2}$

$2 \mathrm{mM} \mathrm{KH}_{2} \mathrm{PO}_{4}$

$5 \mathrm{mM}$ methionine

$10 \mathrm{mM}$ MOPS-KOH pH 7.2

$250 \mathrm{mM}$ sucrose

$1 \mathrm{mM}$ EDTA

$10 \mathrm{mM}$ MOPS $\mathrm{pH} 7.2$

$20 \mathrm{mM} \mathrm{NaH} \mathrm{PO}_{4} / \mathrm{Na}_{2} \mathrm{HPO}_{4}$

$1 \mathrm{M}$ Tris

$1 \mathrm{M}$ Boric Acid

$20 \mathrm{mM}$ EDTA

$\mathrm{MeOH} 40 \%$ (v/v), Acetic acid 10\% (v/v), $\mathrm{H}_{2} \mathrm{O}$ $50 \%(\mathrm{v} / \mathrm{v})$

Coomassie Serva Blue R250 0.15\%

$\mathrm{MeOH} 40 \%$ (v/v), Acetic acid 10\% (v/v), $\mathrm{H}_{2} \mathrm{O}$ $50 \%(\mathrm{v} / \mathrm{v})$

$400 \mathrm{mM}$ Tris-acetate, $10 \mathrm{mM}$ EDTA

\subsubsection{Instruments}

\subsubsection{Centrifuges}

\begin{tabular}{l|l} 
Product & Supplier \\
\hline $5417 \mathrm{R}$ centrifuge & Eppendorf (Hamburg, Germany) \\
5424 centrifuge & Eppendorf (Hamburg, Germany)
\end{tabular}


5804R centrifuge

Sorvall RC 6 Plus

Sorvall RC 12BP

$\mathrm{J} 2-\mathrm{MC}$

Optima TM MAX ultracentrifuge
Eppendorf (Hamburg, Germany)

Thermo Scientific (Bad Homburg, Germany)

Thermo Scientific (Bad Homburg, Germany)

Beckman Coulter (Palo Alto, USA)

Beckman Coulter (Palo Alto, USA)

\subsubsection{Rotors}

Product

F45-30-11

F45-24-11

A-4-44

Sorvall F14S-6x250Y

Sorvall SS-34

Sorvall F10S-6x500Y

Sorvall H-12000

JA-20

TLA-55

\section{Supplier}

Eppendorf (Hamburg, Germany)

Eppendorf (Hamburg, Germany)

Eppendorf (Hamburg, Germany)

Thermo Scientific (Bad Homburg, Germany)

Thermo Scientific (Bad Homburg, Germany)

Thermo Scientific (Bad Homburg, Germany)

Thermo Scientific (Bad Homburg, Germany)

Beckman Coulter (Palo Alto, USA)

Beckman Coulter (Palo Alto, USA)

\subsubsection{Gel systems and Power supply}

\begin{tabular}{l} 
Product \\
\hline Mini PROTEAN ${ }^{\circledR}$ Tetra Cell \\
Mini-Sub cell GT \\
Hoefer SE 600 Ruby TM \\
electrophoresis unit \\
EPS-601 power supply \\
GIBCO-BRL power supply 250EX
\end{tabular}

Ecoline Staredition RE107

GD5040 gel dryer

UV100 Transilluminator

T1 1 UV transilluminator
Supplier

Bio-Rad (Richmond, USA)

Bio-Rad (Richmond, USA)

GE Healthcare (Buckinghamshire, UK)

GE Healthcare (Buckinghamshire, UK)

Life Technologies (Karlsruhe, Germany)

Lauda (Lauda-Königshofen, Germany)

Scie-PLas (Warwickshire, UK)

Biometra (Göttingen, Germany)

Biometra (Göttingen, Germany)

\subsubsection{Photometers}

\begin{tabular}{l|l} 
Product & Supplier \\
\hline $\begin{array}{l}\text { Carry50 Bio UV/visible } \\
\text { Spectrophotometer }\end{array}$ & Varian Inc (Palo Alto, USA)
\end{tabular}


Biophotometer

NanoVue
Eppendorf (Hamburg, Germany)

GE Healthcare (Buckinghamshire, UK)

\subsubsection{Shakers}

\begin{tabular}{|c|c|}
\hline Product & Supplier \\
\hline G25 Incubator shaker & New Burnswick Scientific Co. (Nürtingen, \\
\hline innova ${ }^{\circledR} 44$ incubator shaker & $\begin{array}{l}\text { New Burnswick Scientific Co. (Nürtingen, } \\
\text { Germany) }\end{array}$ \\
\hline innova ${ }^{\circledR} 2300$ platform shaker & $\begin{array}{l}\text { New Burnswick Scientific Co. (Nürtingen, } \\
\text { Germany) }\end{array}$ \\
\hline Excella E10 platform shaker & $\begin{array}{l}\text { New Burnswick Scientific Co. (Nürtingen, } \\
\text { Germany) }\end{array}$ \\
\hline Polymax 1040 shaker & Heidolph (Schwabach, Germany) \\
\hline Shaker DRS-12 & ELMI (Riga, Latvia) \\
\hline
\end{tabular}

\subsubsection{Other instruments}

\begin{tabular}{l|l} 
Product & Supplier \\
\hline Thermomixer Comfort & Eppendorf (Hamburg, Germany) \\
Savant SpeedVac concentrator & GMI (Ramsey, USA) \\
Vortex-GenieZ & Scientific Industries (New York, USA) \\
T-personal PCR machine & Biometra (Göttingen, Germany) \\
pH meter & inoLab (Straubing, Germany) \\
Potter S homogenizer & Sartorius (Göttingen, Germany) \\
Storm ${ }^{\text {TM }} 820$ PhosphorImager & GE Healthcare (Buckinghamshire, UK) \\
Fluorescence scanner FLA-9000 & Fujifilm (Düsseldorf, Germany) \\
ÄKTApurifier system & GE Healthcare (Münich, Germany) \\
Superose 6 10/300 GL & GE Healthcare (Münich, Germany) \\
LAS 3000 & Fujifilm (Düsseldorf, Germany)
\end{tabular}

\subsubsection{Kits}

\begin{tabular}{l} 
Product \\
\hline Wizard $®$ Plus SV Minprep DNA \\
Purification System \\
Wizard® SV gel and PCR clean up \\
System \\
HiSpeed $®$ Plasmid Midi Kit
\end{tabular}

Supplier

Promega (Madison, USA)

Promega (Madison, USA)

Qiagen (Hilden, Germany) 
mMessage mMachine SP6

MEGAclear ${ }^{\mathrm{TM}}$ Purification of

Transcription Reaction

Amersham $^{\mathrm{TM}}$ Rabbit Reticuloycte

Lysate System for in vitro

translation

Amersham $^{\mathrm{TM}} \mathrm{ECL}^{\mathrm{TM}}$

Mobicol columns

Disposable Polypropylene

Columns

Sulfolink Coupling Gel

ABI PRISM ${ }^{\circledR}$ BigDye ${ }^{\circledR}$

Terminator v1.1 Cycle Sequencing

Kit

KOD HotStart DNA polymerase
Ambion ${ }^{\circledR}$ (Austin, USA)

Ambion ${ }^{\circledR}$ (Austin, USA)

GE Healthcare (Buckinghamshire, UK)

GE Healthcare (Buckinghamshire, UK)

MoBiTec (Göttingen, Germany)

Thermo Scientific (Bad Homburg, Germany)

Pierce (Rockford, USA)

Applied Biosystems (Darmstadt, Germany)

Novagen, Merck (Darmstadt, Germany)

\subsubsection{Enyzmes}

\begin{tabular}{l|l} 
Product & Supplier \\
\hline Restriction Endonucleases & New England Biolabs (Ispwich, USA) \\
DNaseI & Roche (Manheim, Germany) \\
T4 DNA Ligase & New England Biolabs (Ispwich, USA) \\
Zymolyase & Seikagaku Biobusiness (Tokyo, Japan) \\
Proteinase K (PK) & Roche (Manheim, Germany) \\
Creatine Kinase (CK) & Roche (Manheim, Germany)
\end{tabular}

\subsubsection{Antibodies}

Table 1. Details of various antibodies used in this study

\begin{tabular}{l|l|l} 
Primary Antibody & Organism & Source \\
\hline$\alpha$-Atp2 & Yeast & AG Rehling (861) \\
$\alpha$-ATP2 & Human & Invitrogen \\
$\alpha$-Atp5 & Yeast & AG Rehling (1546) \\
$\alpha-$ Atp20 & Yeast & AG Rehling (1517) \\
$\alpha-$-HCHD3 & Human & Abcam \\
$\alpha-C o x 1$ & Yeast & AG Rehling (1538) \\
$\alpha-C o x 2$ & Yeast & AG Rehling (1948) \\
$\alpha-C o x 4$ & Yeast & AG Rehling (578) \\
$\alpha-C O X 6 A 1$ & Human & AG Rehling (3283) \\
$\alpha-C O X$ & Human & Invitrogen \\
$\alpha-C y t 1$ & Yeast & AG Rehling (540) \\
$\alpha-F_{1} F_{O} A T P a s e$ & Human & Invitrogen \\
$\alpha-F c j 1$ & Yeast & AG Rehling (857)
\end{tabular}




\begin{tabular}{l|l|l}
$\alpha$-HSPA9 & Human & Abcam \\
$\alpha$-Hsp70 & Yeast & AG Rehling (657) \\
$\alpha$-IMMT (Mitofilin) & Human & Abcam \\
$\alpha$-MINOS1 & Human & Abcam \\
$\alpha$-Mio10 & Yeast & AG Rehling (3459) \\
$\alpha$-TACO1 & Human & AG Rehling (3627) \\
$\alpha$-Tim21 & Yeast & AG Rehling (258) \\
$\alpha$-TIM23 & Human & AG Rehling (1526) \\
$\alpha$-Tom70 & Yeast & AG Rehling (657) \\
$\alpha$-TOM70 & Human & AG Rehling (3280) \\
$\alpha-$ Rip1 & Yeast & AG Rehling (543) \\
& & \\
Secondary Antibody & Organism & Source \\
Goat $\alpha$-Rabbit & - & Dianova \\
Alexa Fluor & - & Inivitrogen
\end{tabular}

\subsubsection{Yeast strains}

Table 2. Details of yeast strains used in this study including genotype and source.

\begin{tabular}{|c|c|c|}
\hline Strain & Genotype & Source \\
\hline BY4741 & Mat a, his3- $\Delta 1$ leu $2 \Delta 0$ met $15 \Delta 0$ ura $3 \Delta 0$ & $\begin{array}{l}\text { Sikorski and Hieter, } \\
1989\end{array}$ \\
\hline YPH499 & $\begin{array}{l}\text { Mat a, ade2-101 his3- } \Delta 200 \text { leu2- } \Delta 1 \text { ura3-52 } \\
\text { trp1- } \Delta 63 \text { lys2-801 }\end{array}$ & $\begin{array}{l}\text { Sikorski and Hieter, } \\
1989\end{array}$ \\
\hline $\operatorname{atp} 20 \Delta$ & $\begin{array}{l}\text { Mat a, his } 3-\Delta 1 \text { leu } 2 \Delta 0 \text { met } 15 \Delta 0 \text { ura } 3 \Delta 0 \text {; } \\
\text { atp } 20:: \operatorname{kanMX} 4\end{array}$ & Open Biosystems \\
\hline $\operatorname{atp} 2 \Delta$ & $\begin{array}{l}\text { Mat a, his3- } \Delta 1 \text { leu2 } 20 \text { met } 15 \Delta 0 \text { ura } 3 \Delta 0 \text {; } \\
\text { atp2::kanMX4 }\end{array}$ & Open Biosystems \\
\hline$f c j 1 \Delta$ & $\begin{array}{l}\text { Mat a, his3- } \Delta 1 \text { leu2 } \Delta 0 \text { met } 15 \Delta 0 \text { ura } 3 \Delta 0 \\
f_{c j 1:: \operatorname{kanMX} 4}\end{array}$ & Open Biosystems \\
\hline Fcj $1^{Z Z}$ & $\begin{array}{l}\text { Mat a, ade2-101 his3- } \Delta 200 \text { leu2- } \Delta 1 \text { ura3-52 } \\
\text { trp1- } \Delta 63 \text { lys2-801; } f_{c j} 1:: \text { FCJ1-ZZ-HIS3MX6 }\end{array}$ & $\begin{array}{l}\text { Von der Malsburg et } \\
\text { al., 2011; Alkhaja et } \\
\text { al., } 2012\end{array}$ \\
\hline miol0s & $\begin{array}{l}\text { Mat a, his3- } \Delta 1 \text { leu2 } \Delta 0 \text { met } 15 \Delta 0 \text { ura } 3 \Delta 0 \text {; } \\
\text { mio10::kanMX4 }\end{array}$ & Open Biosystems \\
\hline Atp $20^{\mathrm{ZZ}}$ & $\begin{array}{l}\text { Mat a, ade2-101 his3- } \Delta 200 \text { leu2- } \Delta 1 \text { ura3-52 } \\
\text { trp1- } \Delta 63 \quad \text { lys2-801; atp } 20:: \text { ATP20-ZZ- } \\
\text { HIS3MX6 }\end{array}$ & $\begin{array}{l}\text { This study; Alkhaja } \\
\text { et al., } 2012\end{array}$ \\
\hline $\operatorname{Atp} 5^{\mathrm{SF}}$ & $\begin{array}{l}\text { Mat a, ade2-101 his3- } \Delta 200 \text { leu2- } \Delta 1 \text { ura3-52 } \\
\text { trp1- } \Delta 63 \text { lys2-801; atp 5::ATP5-SF-HIS3MX6 }\end{array}$ & This study \\
\hline Atp $20^{\mathrm{SF}}$ & $\begin{array}{l}\text { Mat a, ade2-101 his3- } \Delta 200 \text { leu2- } \Delta 1 \text { ura3-52 } \\
\text { trp1- } \Delta 63 \text { lys2-801; atp } 5: \text { :ATP20-SF- } \\
\text { HIS3MX6 }\end{array}$ & This study \\
\hline Mio $10^{\mathrm{SF}}$ & $\begin{array}{l}\text { Mat a, ade2-101 his3- } \Delta 200 \text { leu2- } \Delta 1 \text { ura3-52 } \\
\text { trp1- } \Delta 63 \text { lys2-801; mio } 10:: \text { MIO10-SF- } \\
\text { HIS3MX6 }\end{array}$ & $\begin{array}{l}\text { This study; Alkhaja } \\
\text { et al. } 2012\end{array}$ \\
\hline
\end{tabular}




\subsubsection{Yeast growth medium}

\begin{tabular}{l|l} 
Medium & Recipe \\
\hline YPG & $1 \%(\mathrm{w} / \mathrm{v})$ yeast extract \\
& $2 \%(\mathrm{w} / \mathrm{v})$ bactopeptone \\
& $3 \%(\mathrm{w} / \mathrm{v})$ glycerol \\
YPD & $1 \%(\mathrm{w} / \mathrm{v})$ yeast extract \\
& $2 \%(\mathrm{w} / \mathrm{v})$ bactopeptone \\
& $2 \%(\mathrm{w} / \mathrm{v})$ glucose \\
YPS & $1 \%(\mathrm{w} / \mathrm{v})$ yeast extract \\
& $2 \%(\mathrm{w} / \mathrm{v})$ bactopeptone \\
SD & $2 \%(\mathrm{w} / \mathrm{v})$ sucrose \\
& $0.67 \%(\mathrm{w} / \mathrm{v})$ yeast nitrogen base \\
& $0.7 \%(\mathrm{w} / \mathrm{v})$ drop out mix of CSM-URA \\
& $20 \mu \mathrm{g} / \mathrm{ml}$ uracil \\
SG & $2 \%(\mathrm{w} / \mathrm{v})$ glucose \\
& $0.67 \%(\mathrm{w} / \mathrm{v})$ yeast nitrogen base \\
& $0.7 \%(\mathrm{w} / \mathrm{v})$ drop out mix of CSM-URA \\
& $20 \mu \mathrm{g} / \mathrm{ml}$ uracil \\
& $2 \%(\mathrm{w} / \mathrm{v})$ glucose
\end{tabular}

\subsubsection{Bacterial medium}

LB medium

LB-Agar Plates
$1 \%(\mathrm{w} / \mathrm{v})$ tryptone

$0.5 \%(\mathrm{w} / \mathrm{v})$ yeast extract

$170 \mathrm{mM} \mathrm{NaCl}$

LB medium with $1.5 \%(\mathrm{w} / \mathrm{v})$ agar

\subsubsection{Bacterial strains}

XL1-Blue

SupE44 hsdR17 recAl endA1 gyrA46 thi rela1 lac$F^{\prime}[$ proAB+lacIq lacZ $\Delta M 15$ Tn10(tetr)]

\subsubsection{Miscellaneous products}

\begin{tabular}{l|l} 
Product & Supplier \\
\hline $\begin{array}{l}\text { Polyvinylidene difluoride (PVDF) } \\
\text { membranes }\end{array}$ & Millipore (Schwalbach, Germany) \\
Whatman paper & $\begin{array}{l}\text { Heinemann (Schwäbisch Gmünd, Germany) } \\
\text { Bio-Rad (Richmond, USA) }\end{array}$ \\
Protein Standards SDS-PAGE & $\begin{array}{l}\text { Amersham, GE Healthcare (Buckinghamshire, } \\
\text { Protein Standards BN-PAGE }\end{array}$ \\
USA)
\end{tabular}


Falcon tubes

Eppendorf tubes

Strep-Tactin Sepharose

Strataclean

IgG Sepharose

FLAG Agarose

Flag peptide

Protein A Sepharose (PAS)
Germany)

BD Biosciences Cell Culture (Sparks, USA)

Eppendorf (Hamburg, Germany)

IBA GmbH (Göttingen, Germany)

Agilent Technologies (Santa Clara, USA)

GE Healthcare (Buckinghamshire, UK)

Sigma (Steinheim, Germany)

Sigma (Steinheim, Germany)

Pierce (Rockford, USA)

\subsection{Growth of biological systems}

\subsubsection{Cultivation of bacteria}

Bacteria were cultivated under standard conditions at $37^{\circ} \mathrm{C}$ with addition of appropriate antibiotics. Ampicillin was added to a final concentration of $100 \mathrm{mg} / \mathrm{L}$.

\subsubsection{Cultivation of yeast}

For precultures, yeast colonies were picked and incubated in a 5-10 $\mathrm{ml}$ YPD preculture in a shake flask and incubated with vigorous shaking overnight at $30^{\circ} \mathrm{C}$. This culture was then used to inoculate main cultures in appropriate medium in shake flasks for various applications with varying final $\mathrm{OD}_{600}$.

\subsubsection{Yeast cryo stock}

Yeast strains were preserved as cryo stocks containing YPAD medium (1\% yeast extract, $2 \%$ peptone, $0.003 \%$ adenine sulfate, $2 \%$ glucose, and $15 \%$ glycerol). Yeast cells grown on plate were mixed with YPAD medium in previously autoclaved cryo tubes and stored at $-80^{\circ} \mathrm{C}$.

\subsubsection{Growth analysis of yeast}

Yeast growth tests were performed by adjusting cultured yeast to an $\mathrm{OD}_{600}$ of 0.1 and spotting of serial dilutions onto agar plates containing $1 \%$ yeast extract, $2 \%$ peptone, with 3\% glycerol (for YPG) or 2\% glucose (YPD). Alternatively, plates containing synthetic medium were used: $0.67 \%$ yeast nitrogen base (Difco, BD), $0.7 \mathrm{~g} / 1$ drop-out mix of CSM-URA (MP biomedicals), $20 \mu \mathrm{g} / \mathrm{ml}$ of uracil, supplemented with $2 \%$ 
glucose (SD) or 3\% gylcerol (SG).

\subsubsection{Cultivation of HEK293T cells}

HEK293T cells were cultured under standard condition in Dulbecco's Modified Eagle Medium containing $10 \%$ fetal bovine serum at $37^{\circ} \mathrm{C}$ and $5 \% \mathrm{CO}_{2}$. For SILAC analysis, cells were grown in lysine- and arginine-deficient DMEM Medium supplemented with $10 \%$ fetal bovine serum. Light labeled medium was supplemented with normal isotope containing L-lysine and L-arginine, whereas heavy labeled medium was supplemented with heavy isotope labeled ${ }^{13} \mathrm{C}_{6}{ }^{15} \mathrm{~N}_{2}$-lysine and ${ }^{13} \mathrm{C}_{6}{ }^{15} \mathrm{~N}_{4}$-arginine (Euriso-Top, Germany).

\subsection{Molecular Biology Techniques}

\subsubsection{PCR amplification}

PCR amplification was performed using yeast genomic DNA and the KOD HiFi DNA polymerase (KOD HotStart, Novagen).

\subsubsection{Determination of nucleic acid concentrations}

DNA and RNA concentrations were determined photometrically using NanoVue (GE Healthcare). In brief, 1-2 $\mu 1$ of nucleic acid was added to the NanoVue and quantified accordingly at appropriate wavelength and settings.

\subsubsection{DNA electrophoresis}

PCR products and plasmids were analyzed by agarose gel electrophoresis. In brief, a gel solution composed of $0.7-1.2 \%(\mathrm{w} / \mathrm{v})$ agarose (Agarose NEEO Ultra-Roti, Roth) in 1x TAE buffer gel solution with ethidium bromide was prepared and poured into UVtransparent gel trays inserted into the gel casters. Gel solutions were allowed to polymerize after the addition of well forming combs. After loading all test samples and DNA size marker (Geneladder ${ }^{\mathrm{TM}}$ DNA Marker Mix, Fermentas), gels were subjected to a voltage of 100-120 mV. Upon completion of electrophoresis, a UV100 Transilluminator or alternatively a Biometra T1 1 UV transilluminator was used to visualize DNA. 


\subsubsection{Sequencing DNA}

Sequencing was performed by dideoxy chain termination method (Sanger et al., 1977). DNA template or plasmid samples were combined with 8 pmol PCR primer, $15 \% \mathrm{v} / \mathrm{v}$ sequencing mix and $15 \% \mathrm{v} / \mathrm{v}$ sequencing buffer (ABI PRISM ${ }^{\circledR}$ BigDye ${ }^{\circledR}$ Terminator v1.1 Cycle Sequencing Kit). For sequencing templates from PCR products, the sequencing mix and sequencing buffer solutions were reduced to $10 \% \mathrm{v} / \mathrm{v}$. Templates were amplified by PCR for $25-30$ cycles $\left(96^{\circ} \mathrm{C}\right.$ melting for 10 seconds, $55^{\circ} \mathrm{C}$ annealing for 15 seconds and extension at $60^{\circ} \mathrm{C}$ for 4 minutes) and consequently precipitated in $48 \mathrm{mM}$ EDTA, 80\% ethanol followed by washing with $70 \%$ ethanol and drying DNA pellets in a SpeedVac for 2 minutes. Dried pellets were resuspended in $15 \mu 1$ formadine (HiDye). Samples were analyzed by a Genetic Analyzer 3100 (Applied Biosystems) and DNA sequences analyzed by the program ApE (M. Wayne Davis).

\subsubsection{Transformation of E.coli}

Frozen heat-competent bacteria cell were thawed on ice and mixed with 30 ng DNA and incubated for 20 minutes. Cells were then heat-pulsed at $42^{\circ} \mathrm{C}$ for 45 seconds before a further 2 minute incubation on ice. Pre-warmed LB medium was added to each sample (9x volume) and cells were incubated for 1 hour at $37^{\circ} \mathrm{C}$ with gentle shaking. Approximately $100 \mu \mathrm{l}$ of each transformation was plated on LB agar plates containing appropriate antibiotic (Ampicillin + LB agar plates with $100 \mathrm{mg} / \mathrm{L}$ Ampicillin). Plates were incubated overnight at $37^{\circ} \mathrm{C}$ or until appearance of colonies.

\subsubsection{Transformation of yeast}

Yeast cells grown overnight in YPD were pelleted and washed with distilled $\mathrm{H}_{2} \mathrm{O}$ before treatment with $0.1 \mathrm{M}$ lithium acetate to generate transformation competent cells. Transformation reactions were carried out by the addition of: $10 \%(\mathrm{v} / \mathrm{v})$ carrier DNA (Herring sperm) and 5-10\% (v/v) PCR product or alternatively $2 \%(\mathrm{v} / \mathrm{v})$ plasmid and mixed with 5x volume of 40\% PEG-4000 in lithium acetate. Samples were incubated for 30 minutes at $30^{\circ} \mathrm{C}$ after which, DMSO was added to a final concentration of $10 \%(\mathrm{v} / \mathrm{v})$ and samples incubated for a further $20 \mathrm{~min}$ at $42^{\circ} \mathrm{C}$ with gentle shaking. After incubation, samples were centrifuged at $4000 \mathrm{rpm}$ for $5 \mathrm{~min}$. Pellets were resuspended in YPD and incubated with shaking for $2-4$ hours at $30^{\circ} \mathrm{C}$ 
and then washed with distilled $\mathrm{H}_{2} \mathrm{O}$ before plating on selective medium plates and incubated at $30^{\circ} \mathrm{C}$ until appearance of colonies.

\subsubsection{Yeast genomic DNA preparation}

Yeast cells were grown to an $\mathrm{OD}_{600}$ of 0.5-0.7, pelleted, and resuspended in Solution A (50 mM Tris/HCL pH 7.5, 10 mM EDTA, 0.3\% ß-mercaptoethanol, 0.5-0.25 mg/ml Zymolyase) and incubated for 1 hour at $37^{\circ} \mathrm{C}$. Consequentially, SDS was added to a final concentration of $1 \%(\mathrm{v} / \mathrm{v})$ together with ammonium acetate and incubation at $20^{\circ} \mathrm{C}$ for $15 \mathrm{~min}$. Samples were pelleted, and approximately $66 \%$ of the supernatant was saved and incubated with isopropanol (40\% v/v). Samples were again centrifuged and the resulting pellet was washed in $70 \%$ ethanol and dried. The dried pellet was finally resuspended in TE buffer.

\subsection{Protein Biochemistry}

\subsubsection{TCA precipitation}

Samples were treated with $0.2 \mathrm{x}$ of final volume with $72 \% \mathrm{TCA}(\mathrm{w} / \mathrm{v})$ stock solution and $0.01 \times(1 / 100)$ of sodium deoxycholate (1.25\% stock solution). All samples were incubated $30 \mathrm{~min}$ on ice followed by centrifugation at $4^{\circ} \mathrm{C}$ for $30 \mathrm{~min}$. The resulting pellets were washed with $500 \mu \mathrm{l}$ ice-cold acetone followed by centrifugation at $4^{\circ} \mathrm{C}$ for $30 \mathrm{~min}$. Pellets were dried at $37^{\circ} \mathrm{C}$ for $5-10 \mathrm{~min}$ and then resuspended in $1 \mathrm{x}$ SDSPAGE sample buffer and incubated at $60^{\circ} \mathrm{C}$ for $15 \mathrm{~min}$ before SDS-PAGE analysis.

\subsubsection{Whole cell extracts}

Cultured yeast cells, grown to an $\mathrm{OD}_{600}$ of 3 , were pelleted, and resuspended in $1 \mathrm{ml}$ $\mathrm{H}_{2} \mathrm{O}$ with $250 \mathrm{mM} \mathrm{NaOH}$ and $1 \%(\mathrm{v} / \mathrm{v}) \beta$-mercaptoethanol. Following a $10 \mathrm{~min}$ incubation on ice, proteins were TCA precipitated by the addition of $6 \%(\mathrm{v} / \mathrm{v})$ TCA and incubation at $10 \mathrm{~min}$ on ice, followed by centrifugation at $14,000 \mathrm{rpm}$ at $4^{\circ} \mathrm{C}$. The protein containing pellet was further resuspended in SDS sample buffer and $\mathrm{pH}$ titrated with $1 \mathrm{M}$ Tris-Base, followed by incubation at $60^{\circ} \mathrm{C}$ before subjecting samples to SDS-PAGE analysis. 


\subsubsection{SDS-PAGE analysis}

For SDS-PAGE, components were mixed as shown in Table 3. Glass walls, spacers and combs were cleaned with $70 \%$ ethanol and assembled on a holder. A separating gel was poured between two glass plates and overlain with isopropanol until polymerized followed by pouring of the stacking gel and insertion of appropriate combs. After polymerization, combs were removed and the gel apparatus was placed into an electrophoresis chamber filled with running buffer. Large gels were run at a constant current of $30 \mathrm{~mA}$ and $220 \mathrm{mV}$, whereas Mini-gels were run a constant current of $25 \mathrm{~mA}$ and $100 \mathrm{mV}$. For the analysis of Mio10 import, a Tricine-SDS PAGE was used (Schägger and von Jagow, 1987). A 48\% w/v acrylamide, $1.5 \% \mathrm{w} / \mathrm{v}$ bisacrylamide solution was used. A 3x gel buffer (3 M Tris, 0.3\%, pH $8.45 / \mathrm{HCl}$ ) in used in prepration of the gel which contained a 4\% stacking, $16.5 \%$ separating gel, and a $10 \%$ spacer between stacking and separating gel. In addition, a 10x cathode buffer (1 $\mathrm{M}$ Tris, $1 \mathrm{M}$ Tricine, 1\% SDS, $\mathrm{pH} 8.25)$ and a 10x anode buffer (2 M Tris, $\mathrm{pH} 8.9 /$ $\mathrm{HCl}$ ) were used. Gels were run slowly overnight at $80 \mathrm{mV}$ or $18 \mathrm{~mA}$ limiting.

Table 3. SDS-PAGE pipetting scheme. Constituents of SDS gel were added as indicated to make 1 SDS-PAGE gel.

\begin{tabular}{|l|l|l|l|}
\cline { 2 - 4 } \multicolumn{1}{c|}{} & \multicolumn{2}{l|}{ Resolving Gel } & Stacking Gel \\
\hline \% Acrylamide & $14 \%$ & $12.5 \%$ & \\
\hline $\begin{array}{l}\text { Acrylamide/ bisacrylamide } \\
(30 / 0.8)\end{array}$ & $7.9 \mathrm{ml}$ & $6.9 \mathrm{ml}$ & $0.83 \mathrm{ml}$ \\
\hline $1.87 \mathrm{M}$ Tris pH 8.8 & $3.5 \mathrm{ml}$ & $3.5 \mathrm{ml}$ & - \\
\hline $0.8 \mathrm{M}$ Tris pH 6.8 & - & - & $0.5 \mathrm{ml}$ \\
\hline $10 \%(\mathrm{w} / \mathrm{v}) \mathrm{SDS}$ & 0.17 & $0.17 \mathrm{ml}$ & $50 \mu \mathrm{l}$ \\
$\mathrm{ml}$ & $5.3 \mathrm{ml}$ & $6.3 \mathrm{ml}$ & $3.55 \mathrm{ml}$ \\
\hline $\mathrm{dH}_{2} \mathrm{O}$ & $100 \mu \mathrm{l}$ & $100 \mu \mathrm{l}$ & $50 \mu \mathrm{l}$ \\
\hline $10 \%(\mathrm{w} / \mathrm{v})$ APS & $10 \mu \mathrm{l}$ & $10 \mu \mathrm{l}$ & $10 \mu \mathrm{l}$ \\
\hline TEMED & $17 \mathrm{ml}$ & $17 \mathrm{ml}$ & $5 \mathrm{ml}$ \\
\hline Total Volume & & & \\
\hline
\end{tabular}

\subsubsection{BN-PAGE Analysis}

\subsubsection{First Dimension BN-PAGE}

Prior to gel pouring and preparation, all components shown in Table 4 except for APS and TEMED were mixed accordingly and stored at $4{ }^{\circ} \mathrm{C}$. Glass walls, spacers and 
combs were cleaned with ethanol and assembled on a holder. A gradient separating gel was poured accordingly and overlain with isopropanol. Stacking gel was poured and combs inserted. Polymerization of BN-PAGE gel was performed overnight at $4{ }^{\circ} \mathrm{C}$ or alternatively for 1 hour at RT. BN-PAGE samples were loaded at $4{ }^{\circ} \mathrm{C}$ and highmolecular weight markers used, with 1x BN-PAGE loading dye used to fill in empty lanes. The gel apparatus is then transferred into the BN-PAGE electrophoresis chamber containing $4 \mathrm{~L}$ pre-cooled 1x Anode Buffer and $500 \mathrm{ml}$ Cathode Buffer added. Gels were run at $4^{\circ} \mathrm{C}$ first at a constant voltage of $200 \mathrm{mV}$ at the region of stacking gel and then $600 \mathrm{mV}$ at the separating gel with a constant current of $30 \mathrm{~mA}$. Alternatively, gels were set to $100 \mathrm{mV}$ and run overnight. In the case of subsequent blotting, second dimension analysis or activity assays, the cathode buffer is exchanged, with 1x Cathode buffer containing no Coomassie Serva Blue G250, 1 hour after electrophoresis.

\subsubsection{Second Dimension BN-PAGE}

BN PAGE was performed as described above (with cathode buffer exchange). After electrophoresis gel stripes of individual lanes were excised accordingly and incubated in $\mathrm{H}_{2} \mathrm{O}$. Gel stripes were then placed on the upper part of an SDS-PAGE glass plate, approximately $15 \mathrm{~mm}$ above the expected stacking/separating gel boundary. Glass plates were assembled and separating and stacking gel solutions were poured. A 2-3 comb well was used to load markers and/or mitochondria controls. SDS-PAGE was performed as described above followed by Western blotting.

Table 4. BN-PAGE pipetting scheme. Constituents of BN gel were added as indicated to make $1 \mathrm{BN}-\mathrm{PAGE}$ gel.

\begin{tabular}{|l|l|l|l|l|}
\cline { 2 - 5 } \multicolumn{1}{c|}{} & \multicolumn{3}{c|}{ Resolving Gel } & Stacking Gel \\
\hline \% Acrylamide & 4 & 10 & 13 & \\
\hline 3x Gel Buffer & $3 \mathrm{ml}$ & $3 \mathrm{ml}$ & $3 \mathrm{ml}$ & $2.5 \mathrm{ml}$ \\
\hline Acrylamide & $0.73 \mathrm{ml}$ & $1.82 \mathrm{ml}$ & 2.35 & $0.6 \mathrm{ml}$ \\
\hline Glycerol & - & $1.8 \mathrm{ml}$ & $1.8 \mathrm{ml}$ & - \\
\hline $10 \%$ APS (w/v) & $40 \mu 1$ & $40 \mu 1$ & $40 \mu 1$ & 30 \\
\hline TEMED & $4 \mu \mathrm{l}$ & $4 \mu 1$ & $4 \mu \mathrm{l}$ & 3 \\
\hline $\mathrm{dH}_{2} \mathrm{O}$ & $5.228 \mathrm{ml}$ & $2.347 \mathrm{ml}$ & $1.817 \mathrm{ml}$ & 4.367 \\
\hline
\end{tabular}




\subsubsection{Coomassie staining}

After electrophoresis, gels were stained in gel staining solution for 1 hour and then destained in gel destaining solution for 1 hour.

\subsubsection{Western blotting and immunodetection}

Western blots were performed with PVDF membranes and detected by enhanced chemiluminescence (ECL). In brief, PVDF membranes were incubated in methanol prior to blot and blotting was performed at $250 \mathrm{~mA}$ and $40 \mathrm{~V}$ for $2 \mathrm{hrs}$. After blotting, PVDF membranes were incubated in Staining solution for 30 seconds and washed with Destaining solution. Marker bands were marked and membrane washed with Destaining solution and methanol until clear. For SDS-PAGE analysis, membranes were blocked with 5\% Milk-TBST for 1 hour at RT and incubated with appropriate antibody for 1 hour at RT followed by $3 \times 15$ min washes with TBST. Secondary antibody was applied for 1 hour at RT followed by 3x 15 min washes with TBST, and finally detection was performed by ECL. For BN-PAGE immunodetection, membranes were blocked with 10\% Milk-TBST for 4 hrs or overnight and primary antibody and secondary antibody incubations were for 1 hour each at RT and detection was by ECL and a film developing machine. Alternatively, fluorescently labeled antibodies were used and detection was performed with the fluorescence scanner (FLA-9000; Fujifilm).

\subsubsection{Size exclusion chromatography}

Solubilized yeast and human mitochondrial protein complexes were separated by gel filtration using an ÄKTApurifier system (GE Healthcare). In brief, $200 \mu \mathrm{g}$ of isolated mitochondria were solubilized in $200 \mu \mathrm{l} \mathrm{GF-buffer}(20 \mathrm{mM}$ Tris/pH7.4, $50 \mathrm{mM} \mathrm{NaCl}$, $0.5 \mathrm{mM}$ EDTA, 10\% (w/v) Glycerol, 1\% (w/v) Digitonin, 1mM PMSF) for $20 \mathrm{~min}$ on ice. Samples were centrifuged, insoluble pellet discarded and resulting supernatants were loaded on a Superose 6 10/300 GL (GE Healthcare) equilibrated with GL-buffer (20 mM Tris/pH7.4, 50 mM NaCl, 0.5 mM EDTA, 10\% (w/v) Glycerol, 0.1\% (w/v) Digitonin). The resulting fractions from the ÄKTApurifier system were precipitated with TCA as described above with the exception of a further acetone wash prior to drying and resolving samples in 1x SDS sample buffer. Samples were then analyzed by SDS-PAGE and Western blotting. 


\subsubsection{Digital autoradiography}

For detection and analysis of radioactive signals by digital autoradiography, destained gels are first placed on Whatman paper, covered with a plastic sheet and dried for 2 hours. After drying, markers on the gel are labeled with radioactive ink and covered with transparent tape. The gel is placed in an autoradiography cassette and film is exposed accordingly.

\subsubsection{Isolation of mitochondria from yeast}

\subsubsection{Mitochondrial preparation $Y P G$ at $30^{\circ} \mathrm{C}$}

Yeast mitochondria were isolated by differential centrifugation as described by Meisinger et al., 2006. For each strain, the preculture was used to inoculate 5.1-10.2 L medium to achieve an $\mathrm{OD}_{600} \mathrm{~nm}$ of 2.0 , in which yeast should be a mid-log growth phase at time of harvest. Main cultures were grown for 15-18 hrs with vigorous shaking at $30^{\circ}$. Yeast cells were harvested by centrifugation at $4000 \mathrm{rpm}$ in a RC 12BP Sorvall centrifuge for $20 \mathrm{~min}$ at $18^{\circ} \mathrm{C}$ and supernatant was discarded. The pellets were resuspended in water and combined in a F10S centrifugation tube, and centrifuged in a Sorvall F10S-6x500Y rotor at $4000 \mathrm{rpm}$ for $10 \mathrm{~min}$ at $18^{\circ} \mathrm{C}$. Supernatant was discarded and the weight of wet cell pellet was determined. The resulting cell pellets were resuspended in DTT buffer (100 mM Tris/HCl $\mathrm{pH} 9.4,10 \mathrm{mM}$ DTT) at a concentration of $2 \mathrm{ml}$ per gram yeast and incubated with shaking at $30^{\circ} \mathrm{C}$ for $30 \mathrm{~min}$. Cell suspensions were centrifuged in a Sorvall F10S-6x500Y rotor at $4000 \mathrm{rpm}$ at $18^{\circ} \mathrm{C}$ for $8 \mathrm{~min}$. Supernatants were discarded and pellets were washed with $200 \mathrm{ml} 1.2$ M Sorbitol and cell suspension was centrifuged at $4000 \mathrm{rpm}$ at $18^{\circ} \mathrm{C}$ for $8 \mathrm{~min}$ (Sorvall F10S-6x500Y rotor). Supernatants were again discarded and pellets resuspended in zymolyase buffer (20 mM KPi Buffer, 1.2 M Sorbitol), supplemented with $4 \mathrm{mg}$ zymolyase per gram of yeast. Cell suspensions were incubated with shaking at $30^{\circ} \mathrm{C}$ for $60 \mathrm{~min}$ and then centrifuged at $3000 \mathrm{rpm}$ at $18^{\circ} \mathrm{C}$ for $10 \mathrm{~min}$ (Sorvall F10S-6x500Y rotor). Pellets were washed with zymolyase buffer without enzyme and centrifuged at $3000 \mathrm{rpm}$ at $18^{\circ} \mathrm{C}$ for $10 \mathrm{~min}$ (Sorvall F10S-6x500Y rotor). Supernatants were discarded and pellets were stored on ice and resuspended in Homogenization Buffer (0.6 M Sorbitol, $10 \mathrm{mM}$ Tris $/ \mathrm{HCl} \mathrm{pH} \mathrm{7.4,} 1 \mathrm{mM}$ EDTA, 0.2\% (w/v) BSA) at a concentration of $7 \mathrm{ml}$ per gram yeast. PMSF was added to a final concentration of 1 $\mathrm{mM}$ to each suspension. Cells were homogenized by a Potter S Homogenizer and centrifuged in a Sorvall F15S-6x250Y rotor at $3000 \mathrm{rpm}$ for $5 \mathrm{~min}$ at $4^{\circ} \mathrm{C}$. Pellets were 
discarded and supernatants were transferred into fresh F15S tubes and centrifuged at $4000 \mathrm{rpm}$ for $10 \mathrm{~min}$ at $4^{\circ} \mathrm{C}$. Supernatants were discarded whereas pellets were pooled in SS-34 tubes and washed in $5 \mathrm{ml}$ SEM Buffer with a final concentration of $1 \mathrm{mM}$ PMSF and centrifuged in a Sorvall SS-34 rotor at $12000 \mathrm{rpm}$ for 15 mins at $4{ }^{\circ} \mathrm{C}$. Supernatant was discarded and pellet was resuspended in SEM buffer and protein concentration was determined by the Bradford Assay (Bradford, 1976). Mitochondrial pellets were diluted in SEM buffer to a final concentration of $10 \mu \mathrm{g} / \mu 1$, aliquoted, and stored at $-80^{\circ} \mathrm{C}$.

\subsubsection{Mitochondrial Preparation from $S G$ at $18^{\circ} \mathrm{C}$}

Isolation from yeast grown in $\mathrm{SG}$ at $18^{\circ} \mathrm{C}$ was performed essentially as described above with some variations. For the primary culture, yeast colonies were picked and inoculated into SD medium and grown overnight at $30^{\circ} \mathrm{C}$. Final SG cultures were grown at $30^{\circ} \mathrm{C}$ until $\mathrm{OD}_{600}$ of 1.0 and then transferred to $18^{\circ} \mathrm{C}$ until culture reached $\mathrm{OD}_{600} 2.0$ (generally 24 hours after temperature shift).

\subsubsection{Isolation of mitochondria from HEK 293T cells}

Human mitochondria were isolated from HEK293T cells as previously described (Lazarou et al., 2009; Reinhold et al., 2011). In brief, cells were harvested at 80-85\% confluency in 1x PBS and $1 \mathrm{mM}$ EDTA and homogenized in $0.1 \%$ BSA, $300 \mathrm{mM}$ Trehalose, 10 mM HEPES-KOH pH 7.7, 10 mM KCl, 1 mM EDTA, 1 mM EDTA (Yamaguchi et al., 2007). Homogenized cells were subjected to centrifugation at $11,000 \times \mathrm{g}$ at $4^{\circ} \mathrm{C}$ for $10 \mathrm{~min}$ and homogenization buffer without BSA was added to the mitochondria containing pellet.

\subsubsection{Labeling of precursor proteins with $\left[{ }^{35} \mathrm{~S}\right]$ methionine}

For in vitro translation, ORFs of interest were amplified by PCR as described above. Amplified PCR products containing SP6 promoter sites were transcribed using the mMessage mMachine system. Each reaction mix contained $6 \mu 1$ purified PRC product, 1x SP6 reaction buffer, 1x NTP/CAP mixture and $2 \mu 1$ Enzyme mix, and reactions were incubated for 2 hours at $37^{\circ} \mathrm{C}$ in a thermomixer. After 1 hour, 2 units of TURBO DNase were added and mixture was incubated for $15 \mathrm{~min}$ at $37^{\circ} \mathrm{C}$. RNA sample was cleared by the Ambion Megaclear kit. Labeling of preproteins was performed using the Flexi ${ }^{\circledR}$ Rabbit Reticulocyte Lysate system (Promega). Each translation reaction 
contained 20\% RNA, 66\% rabbit reticuloycte lysate, $70 \mathrm{mM}$ potassium chloride, 1.4 $\mathrm{mM}$ magnesium acetate, and $1.6 \mathrm{mM}$ radioactive methionine. Samples were incubated in a thermomixer at $30^{\circ} \mathrm{C}$ for $90 \mathrm{~min}$, followed by the addition of $150 \mathrm{mM}$ sucrose and non-radiolabeled methionine to a final concentration of $8 \mathrm{mM}$. Lysates were aliquoted accordingly and stored at $-80^{\circ} \mathrm{C}$.

\subsubsection{Import and assembly of radiolabeled precursor protein}

\subsubsection{Import into yeast isolated mitochondria}

Import of radiolabeled proteins into isolated yeast mitochondria were performed as described by Wiedemann et al., 2006. Import reactions were performed in Standard Import Buffer and supplemented with 4 mM ATP, 4 mM NADH, $100 \mu \mathrm{g} / \mathrm{mL}$ creatine kinase, $5 \mathrm{mM}$ creatine phosphate and $75 \mu \mathrm{g}$ isolated mitochondria. For samples with dissipated membrane potentials, $1 \%(\mathrm{v} / \mathrm{v})$ of $100 \mu \mathrm{M}$ AVO was added whereas $1 \%$ (v/v) ethanol was added to other samples. Samples were incubated for $3 \mathrm{~min}$ at $25^{\circ} \mathrm{C}$ and import reactions were initiated with the addition of radiolabeled lysate $(10-15 \%$ of total volume). Imports were stopped at appropriate time points with the addition of 1 $\mu 1100 \mu \mathrm{M}$ AVO and transferred to ice. Reactions were treated with $2.5 \mu \mathrm{g}$ PK and incubated on ice for $15 \mathrm{~min} .1 \mu \mathrm{l} 0.2 \mathrm{M}$ PMSF was added to halt PK activity and samples were centrifuged at $14,000 \mathrm{rpm}$ for $10 \mathrm{~min}$. Mitochondrial pellets were washed with $300 \mu$ l SEM buffer with $2 \mathrm{mM}$ final concentration PMSF and centrifuged at $14,000 \mathrm{rpm}$ for $10 \mathrm{~min}$ at $4^{\circ} \mathrm{C}$. For SDS-PAGE analysis, pellets were resuspended in sample buffer and loaded on gels for electrophoresis. For BN-PAGE analysis, pellets were solubilized in Digitonin buffer $(20 \mathrm{mM}$ Tris $\mathrm{pH} 7.4,50 \mathrm{mM} \mathrm{NaCl}, 0.5 \mathrm{mM}$ EDTA, $10 \%(\mathrm{w} / \mathrm{v})$ Glycerol, $1 \%(\mathrm{w} / \mathrm{v})$ Digitonin, and $1 \mathrm{mM}$ PMSF). After solubilization, samples were centrifuged at $14,000 \mathrm{rpm}$ for $10 \mathrm{~min}$ at $4{ }^{\circ} \mathrm{C}$ and the resulting supernatants were suspended in 10\% (v/v) 1x BN Loading Dye, before loading on BN gels.

\subsubsection{Import into HEK293T isolated mitochondria}

Import of radiolabeled proteins into isolated human mitochondria was performed as described by Lazarou et al. (2009). For import of radiolabeled lysate into mitochondria from isolated mitochondria from HEK293T cells was performed essentially as described above with some notable changes. Import and assembly assays were incubated with freshly isolated mitochondria and not frozen mitochondria. Moreover, 
the import buffer (20 mM HEPES/KOH pH 7.4, $250 \mathrm{mM}$ sucrose, $80 \mathrm{mM}$ potassium acetate, $5 \mathrm{~mm}$ magnesium acetate, $10 \mathrm{~mm}$ sodium succinate and $5 \mathrm{~mm}$ methionine) was used. Import reactions were performed at $37^{\circ} \mathrm{C}$.

\subsubsection{Protein localization analysis}

To determine protein localization within isolated mitochondria, a protease protection assay was performed as previously described by Mick et al. (2007). Mitochondria, mitoplasts, or Triton X-100 lysed mitochondria were treated with various amounts of $\mathrm{PK}$, in which then proteins were tested for localization to the mitochondrial outer membrane, mitochondrial inner membrane or matrix by SDS-PAGE and Western blot analysis. Mitochondria were resuspended in SEM buffer and treated with either 0, 20, or $100 \mu \mathrm{g}$ PK. Swelling of isolated mitochondria was achieved by treatment in EM buffer (1 mM EDTA, $10 \mathrm{mM}$ MOPS pH 7.2). The generated mitoplasts were treated with 0,20 or $100 \mu \mathrm{g}$ PK. Mitochondria lysed with 1\% Triton X-100 were treated with 0 or $100 \mu \mathrm{g}$ PK. In all cases, PK treatment was allowed for $10 \mathrm{~min}$ on ice and followed by the addition of $0.2 \mathrm{M}$ PMSF and incubated on ice for $10 \mathrm{~min}$. All samples were centrifuged at $14,000 \mathrm{rpm}$ at $4^{\circ} \mathrm{C}$. The pellets of SEM-treated mitochondria and mitoplasts and the supernatant of Triton X-100 lysed mitochondria were resuspended in SDS-PAGE loading dye and analysed in by SDS-PAGE.

\subsubsection{Membrane association analysis}

Analysis was performed as previously described (Mick et al., 2007). To test protein membrane association, isolated mitochondria were suspended in $0.1 \mathrm{M}$ carbonate buffer at $\mathrm{pH} 10.8$ or 11.5 , or alternatively lysed with $1 \%$ Triton X-100. All samples were incubated on ice and then subjected to ultracentrifugation at $45000 \mathrm{rpm}$ for 45 min at $4{ }^{\circ} \mathrm{C}$, in a TLA-55 rotor (Beckmann). Samples were TCA precipitated and subjected to SDS-PAGE analysis.

\subsubsection{In-gel activity assays}

Isolated mitochondria were solubilized in digitonin buffer and subjected to BN-PAGE gels, at which, coomassie containing-cathode buffer is exchanged, with $1 \mathrm{x}$ clear cathode buffer after 1 hour of electrophoresis at $200 \mathrm{mV}$. In-gel activity assays were performed on sliced gel stripes or whole gels, and incubated first in appropriate equilibration buffer (as indicated in Table 5) for $15 \mathrm{~min}$ followed by incubation in 
staining buffer at RT or $30^{\circ} \mathrm{C}$ until the appearance of bands. Gel stripes were incubated in $50 \mathrm{ml}$ equilibration buffer and $10 \mathrm{ml}$ staining buffer, whereas whole gels were incubated in $100 \mathrm{ml}$ equilibration and $50 \mathrm{ml}$ staining buffer. A 4-10\% gradient gel was used for the assessment of cytochrome $c$ oxidase, complex $\mathrm{I}$, and $\mathrm{F}_{1} \mathrm{~F}_{\mathrm{O}} \mathrm{ATPase}$ monomer and dimeric forms. Alternatively, a 3-13\% gradient gel was to analyze higher oligomers of $\mathrm{F}_{1} \mathrm{~F}_{\mathrm{O}} \mathrm{ATPase}$.

Table 5. Composition of in-gel activity assay buffers. *Reduction of Cytochrome c $(10 \mathrm{mg} / \mathrm{ml})$ was achieved by resuspending $10 \mathrm{mg}$ Cytochrome $c$ in $1 \mathrm{ml} 50 \mathrm{mM} \mathrm{KPi}$ $\mathrm{pH} 7.4$ and $75 \mu \mathrm{g}$ of Na-dithionite.

\begin{tabular}{|c|c|c|}
\hline & Equilibration buffer & Staining Buffer \\
\hline Complex I & $5 \mathrm{mM}$ Tris $/ \mathrm{HCl} \mathrm{pH} 7.4$ & $\begin{array}{l}5 \mathrm{mM} \text { Tris } / \mathrm{HCl} \mathrm{pH} 7.4 \\
2.5 \quad \mathrm{mg} / \mathrm{ml} \text { NBT } \\
\text { Nitrotetrazoliumchrolide blue) }\end{array}$ \\
\hline $\begin{array}{l}\text { Cytochrome } c \\
\text { oxidase }\end{array}$ & 50 mM KPi pH 7.4 & $\begin{array}{l}50 \mathrm{mM} \text { KPi pH } 7.4 \\
0.5 \mathrm{mg} / \mathrm{ml} \mathrm{DAB}(3,3 \text {, } \\
\text { Diaminobenzidine) } \\
1 \mathrm{mg} / \mathrm{ml} \text { of reduced Cytochrome } \\
\mathrm{c} \text { (bovine heart)* }\end{array}$ \\
\hline $\mathrm{F}_{1} \mathrm{~F}_{\mathrm{O}} \mathrm{ATPase}$ & $\begin{array}{l}35 \mathrm{mM} \text { Tris, } 220 \mathrm{mM} \\
\text { Glycine pH } 8.3\end{array}$ & $\begin{array}{l}35 \mathrm{mM} \text { Tris, } 220 \mathrm{mM} \text { Glycine } \\
\mathrm{pH} 8.3 \\
14 \mathrm{mM} \mathrm{MgSO}_{4} \\
0.2 \% \mathrm{~Pb}\left(\mathrm{NO}_{3}\right)_{2} \\
8 \mathrm{mM} \mathrm{ATP} \\
\end{array}$ \\
\hline
\end{tabular}

\subsubsection{Protein complex isolation by immunoprecipitation}

Isolation of yeast protein complexes was performed as previously described (Geissler et al., 2002). In all cases, mitochondria isolated with tagged nuclear encoded proteins were solubilized in IP-Buffer (20 mM Tris/pH7.4, $50 \mathrm{mM} \mathrm{NaCl}, 0.5 \mathrm{mM}$ EDTA, 10\% (w/v) Glycerol, 1\% (w/v) Digitonin, 1mM PMSF). After centrifugation at 14,000 rpm for $20 \mathrm{~min}$, supernatants were incubated with appropriate column material in Mobicol columns for 1 hour at $4^{\circ} \mathrm{C}$ on an end-over-end shaker. Unbound proteins were removed by centrifugation at $100 \mathrm{x} g$ for $1 \mathrm{~min}$. Columns were then washed 10 times with 20x bead volume of Washing Buffer (20 mM Tris/pH7.4, $100 \mathrm{mM} \mathrm{NaCl}, 5 \mathrm{mM}$ EDTA, 10\% (w/v) Glycerol, 0.3\% Digitonin, and $1 \mathrm{mM} \mathrm{PMSF}$ ), and isolated proteins were eluted accordingly. For Large-scale isolation (1 mg mitochondria), solubilization of mitochondria was carried our in either Falcon tubes or $2 \mathrm{ml}$ Eppendorf tubes. o indicates the appropriate experimental conditions for different isolations. 
Table 6. Experimental conditions for various immunoprecipitation assays. Described buffer were used for native elutions. Alternatively, proteins can be eluted by SDS sample buffer.

\begin{tabular}{|l|l|l|}
\hline Type of tag & Binding column material & Elution Buffer \\
\hline $\begin{array}{l}\text { ZZ } \\
\text { (ProteinA) }\end{array}$ & IgG Sepharose & Glycine $\mathrm{pH} 2.8$ \\
\hline $\begin{array}{l}\text { SF } \\
\text { (Steptavidin- } \\
\text { FLAG) }\end{array}$ & $\begin{array}{l}\text { Strep-Tactin sepharose } \\
\text { (IBA GmbH) }\end{array}$ & $\begin{array}{l}5 \mathrm{mM} \text { Desthio-Biotin, 20 mM Tris-HCl } \\
\mathrm{pH} 7.4,30 \mathrm{mM} \mathrm{NaCl}, 0.2 \mathrm{mM} \text { EDTA }\end{array}$ \\
\hline FLAG & FLAG-agarose & $\begin{array}{l}\text { Flag peptide } 20 \mathrm{mM} \text { Tris/pH7.4, } 100 \mathrm{mM} \\
\text { NaCl, 5 mM EDTA, 10\%(w/v) Glycerol, } \\
0.3 \%(\mathrm{w} / \mathrm{v}) \text { Digitonin, } 1 \mathrm{mM} \text { PMSF), }\end{array}$ \\
\hline
\end{tabular}

\subsubsection{Crosslining antibodies for co-immunoprecipitations}

To crosslink antibodies into sepharose columns for consequent use in coimmunprecipitations, $100 \mu \mathrm{l}$ of Protein A Sepharose (PAS) were added to a Mobicol column and centrifuged for $1000 \mathrm{rpm} 5 \mathrm{~min}$ at RT. The sepharose was then washed twice with $500 \mu 10.1 \mathrm{M}$ KPi buffer $\mathrm{pH}$ 7.4. For each column $100 \mu 1$ serum premixed with $300 \mu 1$ with KPi buffer were added to the column followed by incubation on an end-over-end shaker of 1 hour at RT. Flow-through from the column was saved after a 5 min centrifugation and the column was washed with $500 \mu \mathrm{l} \mathrm{NaBorat}$ and then incubated with NaBorat with $17.5 \mathrm{mg}$ DMP for $30 \mathrm{~min}$ at RT. Residual crosslinker was removed, first by centrifugation for $5 \mathrm{~min}$ at $1000 \mathrm{rpm}$, and then washing with 700 $\mu 11 \mathrm{M}$ Tris-HCl, pH 7.4. Mobicol columns were then incubated for 2 hours in $700 \mu 11$ $\mathrm{M}$ Tris- $\mathrm{HCl}$, pH 7.4 followed by centrifugation for $5 \mathrm{~min}$ at $1000 \mathrm{rpm}$. The column was stored at $4^{\circ} \mathrm{C}$ with $600 \mu \mathrm{PBS}$ with a final concentration of $2 \mathrm{mM}$ sodium azide.

\subsubsection{Co-immunoprecipitation from isolated yeast mitochondria}

Antibodies against various yeast proteins were crosslinked to columns as described above. Isolated mitochondria were solubilized in digitonin buffer and applied to columns. Biochemical isolations were carried out using as for protein complex immunoprecipitations with the exception of eluting with $100 \mathrm{mM}$ glycine $\mathrm{pH} 2.8$. 


\subsubsection{Co-immunoprecipitation from HEK293T cells}

Isolation of protein complexes from human isolated mitochondria was performed either with beads coupled with antibodies raised against the $F_{1} F_{O} A T P a s e(\alpha-$ $\left.\mathrm{F}_{1} \mathrm{~F}_{\mathrm{O}} \mathrm{ATPase}\right)$ or against cytochrome $c$ oxidase $(\alpha$-COX) (Invitrogen). Immunoprecipitation of MINOS1-containing complexes were carried out using C1orf151/MINOS1 antibodies, cross-linked to Protein A/G Agarose (Pierce, Thermoscientific). In brief, $1 \mathrm{mg}$ of isolated mitochondria from HEK293T cells was solubilized in $1 \mathrm{ml}$ IP-buffer (20 mM Tris/pH7.4, $50 \mathrm{mM} \mathrm{NaCl}, 0.5 \mathrm{mM}$ EDTA, 10\% (w/v) Glycerol, 1\% (w/v) Digitonin, $1 \mathrm{mM} \mathrm{PMSF}$ ) for $20 \mathrm{~min}$ on ice. Insoluble material was removed by centrifugation $\left(14.000 \mathrm{rpm}, 20 \mathrm{~min}, 4^{\circ} \mathrm{C}\right)$. The remaining supernatent was incubated with the individual antibody coupled beads for $1 \mathrm{~h}$ at $4^{\circ} \mathrm{C}$ on an end-over-end shaker and centrifuged at $1000 \mathrm{rpm}$ for $1 \mathrm{~min}$ at $4^{\circ} \mathrm{C}$ to remove unbound protein. The pelleted beads were transferred to a Mobicol column and beads were washed 10 times with W-buffer $(20 \mathrm{mM}$ Tris/pH 7.4, $50 \mathrm{mM} \mathrm{NaCl}, 0.5 \mathrm{mM}$ EDTA, 10\%(w/v) Glycerol, 0.3\% (w/v) Digitonin, 1mM PMSF). Samples were finally eluted by SDS-PAGE sample buffer.

\subsection{Analytical Tools}

\subsubsection{In silico analysis and multiple sequence alignments}

In silico analysis was performed using the Saccharomyces Genome Database (SGD). For multiple sequence alignments ClustalW 2.0.11 (Chenna et al., 2003) and geneious $\subset$ (Biomatters, Limited) were used. Color coding was performed by Microsoft Word 2008. Black boxes were used to indicate identical residues in at least four species whereas gray boxes indicate similar amino acids, in accordance with Erdmann et al., 1991. Similarity rules used were $(G=A=S . A=V, V=I=L=M$, $\mathrm{I}=\mathrm{L}=\mathrm{M}=\mathrm{F}=\mathrm{Y}=\mathrm{W}, \mathrm{K}=\mathrm{R}=\mathrm{H}, \mathrm{D}=\mathrm{E}=\mathrm{Q}=\mathrm{N}, \mathrm{S}=\mathrm{T}=\mathrm{Q}=\mathrm{N}$ ). Transmembrane predictions from primary amino acid sequences were performed by TMpred (Hofmann and Stoffel, 1993).

\subsubsection{Mass spectrometry and data analysis}

For the identification of MINOS1 interacting proteins, SILAC (Ong et al., 2002) was used. Mitochondria from HEK29T cells grown in heavy or light isotopes (see Section) 
were isolated and MINOS1 containing complexes were coimmunoprecipitated from the heavy-labeled culture, whereas a control antibody was used for light-labeled cells. The reverse (label-switch) experiment was performed by isolating MINOS1 specific complexes from light-labeled cells, whereas the control was used for heavy-labeled cells. In all cases, elutions from coimmunoprecipitations were mixed before further analysis. Eluted proteins from either Mio10 ${ }^{\mathrm{SF}}$ immunoprecipitations from isolated yeast mitochondria, or MINOS1 co-immunoprecipitations from HEK293T cells, were separated on $4-12 \%$ gradient SDS-PAGE gels (Invitrogen) and stained with Colloidal Coomassie Blue. SDS-PAGE gels were cut into 23 equal gel slices and proteins were in-gel digested with trypsin (Promega) as described previously (Shevchenko et al., 2006). The resulting tryptic peptides from each gel slice were analyzed by nanoflow HPLC (Agilent 1100, Agilent Technologies) coupled to nanoelectrospray LTQOrbitrap XL mass spectrometer (Thermo Fischer Scientific). For yeast Mio $10^{\mathrm{SF}}$ immunoprecipitations, the raw MS files were searched and analyzed with Mascot (Mascot Deamon version 2.2.2, Matrix Science) and Scaffold 3 (Proteome Software) using the $S$. cerevisiae NCBI non-redundant protein database. The normalized fold change ratios were then calculated in Scaffold using the spectral count approach (Liu et al., 2004). For SILAC analysis of MINOS1 immunoprecipitations, the raw MS files were analyzed by MaxQuant (Cox and Mann, 2008) and Mascot using IPI Human protein database (version 3.82). MaxQuant was used to analyze and visualize results with $\mathrm{R}$ as described (Nikolov et al., 2011). Mass spectrometric analysis performed by Monika Raabe and Miroslav Nikolov (AG Urlaub, MPI-bpc, Göttingen).

\subsubsection{Fluorescence microscopy}

\subsubsection{Subcellular localization by immunofluorescence}

Immunofluorescence was performed with African green monkey (Cercopithecus aethiops) kidney epithelial cells (Vero), as previously described (Wurm et al., 2010). In brief, cells were grown on cover slips under standard conditions and then fixed in $8 \%$ paraformaldehyde (PFA) and permeabilized in $0.5 \%(\mathrm{v} / \mathrm{v})$ Triton X-100. Treated cells were then incubated for 1 hour with C1orf151/MINOS1-antibody and the mitochondrial marker Cyclophilin D-antibody at a dilution of 1:400. Cells were then washed cells and incubated for 1 hour with Oregon Green 488 conjugated goat antimouse IgGs (1:1000; Molecular Probes) and KK114 conjugated goat anti-rabbit IgGs (1:200) (Kolmakov et al., 2010). Cells were again washed and stained with DAPI 
staining for $5 \mathrm{~min}$ before embedding in mounting medium. Fluorescence microscopy was performed with a beam scanning confocal microscope (TCS SP5, Leica Microsystems CMS GmbH, Wetzlar Germany) equipped with 1.4 NA oil immersion lenses (63 x; HCX PL APO, Leica). Microscopy was performed by Daniel Jans (AG Jakobs, MPI-bpc).

\subsubsection{Morphology analysis by Life-cell imaging fluorescence}

To visualize mitochondrial networks in yeast, cells were first transformed with a pVT100U-mitoGFP vector (Westermann and Neupert, 2000). Selected colonies were first verified for GFP expression and then cultured overnight in selective SGGMedium $(0.5 \mathrm{~g} / 1$ yeast extract, $6.7 \mathrm{~g} / 1$ yeast nitrogen base, $0.77 \mathrm{~g} / 1$ CSM-URA dropout mix, 30ml/1 Glycerol, $1 \mathrm{~g} / 1$ Glucose). Cultured cells were directly used for fluorescence microscopy with no further manipulation. Images were collected by a DeltaVision microscope (Olympus IX71, Applied Precision) equipped with a 100x objective and a FITC filter and deconvolved with Softworx (Applied Precision) version 3.5.1. 10-15 Z-section images were taken for each image at $0.5 \mu \mathrm{m}$ intervals. Microscopy was performed by Daniel Jans (AG Jakobs, MPI-bpc).

\subsubsection{Electron microscopy}

For analysis of mitochondrial morphology of various mutants, electron microscopy, in which sample specific were vitrified by high pressure freezing or chemical fixed by potassium permanganate before examination.

\subsubsection{High-pressure-freezing}

Yeast cells were grown in liquid YP Lactate medium (10 g/l yeast extract, $20 \mathrm{~g} / 1$ peptone from casein, $23 \mathrm{ml} / \mathrm{L}(+)$-lactic acid solution (85\%), $0.02 \mathrm{~g} / 1$ uracil, $0.02 \mathrm{~g} / 1$ adenine sulfate) at $30^{\circ} \mathrm{C}$. Cultures were allowed to allowed to reach a logarithmic growth phase $\left(\mathrm{OD}_{600}\right.$ approximately 2.0$)$ at which point they are harvested. Cells were transferred onto $150 \mu \mathrm{m}$ aluminum planchette and a Leica HPM100 high pressure freezer (Leica Microsystems, Wetzlar, Germany) was used to vitrify samples followed by embedding in $0.5 \%$ glutharaldehyde, $0.1 \%$ uranylacetate and $5 \% \mathrm{H}_{2} \mathrm{O}$ (Giddings, 2003) using a freeze substitution unit (Leica EM AFS, Leica Microsystems). Samples were warmed to room temperature and the pellet was removed from the planchette 
followed by embedding in expoxid resin (Agar 100, Plano) and polymerization at $+80^{\circ} \mathrm{C}$ for 48 hours. Thin sections $(60 \mathrm{~nm})$ were counterstained with $1 \%$ uranylacetate in methanol and lead citrate and prior to examination with a Philips CM 120 BioTwin transmission electron microscope (Philips Inc., Eindhoven, Netherlands). Microscopy and analysis were performed in collaboration with Dietmar Riedel (Mpi-bpc, Göttingen)

\subsubsection{Chemical fixation}

Chemical fixation of sample specimens with potassium permanganate was essentially performed as described by Erdmann et al., 1989. Yeast cells were first cultured for $12 \mathrm{~h}$ at $18^{\circ} \mathrm{C}$ in liquid SGG medium $(0.5 \mathrm{~g} / 1$ yeast extract, $6.7 \mathrm{~g} / 1$ yeast nitrogen base, $0.77 \mathrm{~g} / 1$ CSM dropout mix, 30ml/1 Glycerol, $1 \mathrm{~g} / 1$ Glucose). Cells were then fixed in 1.5\% potassium permanganate at room temperature for $2 \mathrm{~min}$, and consequentially treated with $1 \%$ uranyl acetate for 2 hours. Fixed cells were then dehydrated in a graded ethanol series and embedded in Epon 812 resin. Ultrathin sections were mounted on Formvar-coated single hole grids, and examination was performed in a Philips EM 300 (Phillips Inc., Eindhoven, Netherlands). Microscopy and analysis were performed in collaboration with Wolfgang Schliebs (Ruhr Universität Bochum, Bochum). 


\section{RESULTS}

\subsection{Identification of a putative $F_{1} F_{O} A T P a s e$ dimerization factor in higher eukaryotes (H. sapiens)}

\subsection{1 in silico analysis: identification of Mio10 and MINOS1}

Various structural subunits of the $\mathrm{F}_{1} \mathrm{~F}_{\mathrm{O}} \mathrm{ATPase}$ were described to be involved in dimerization in various species ranging from simple eukaryotes to mammals (Soubannier et al., 2002; Fronzes et al., 2003). In addition, subunits Atp18, Atp19, Atp20, and Atp21 have been described to play a major role in inducing dimerization in yeast by promoting $\mathrm{F}_{1} \mathrm{~F}_{\mathrm{O}} \mathrm{ATPase}$ homo-monomeric interactions (Arnold et al., 1997, 1998; Soubannier et al., 2002; Paumard et al., 2002a; Wagner et al., 2009, 2010). The presence of these small $F_{O}$ associated dimerization factors does not seem to be conserved in higher eukaryotes. Whereas homologs of subunits Atp18 and Atp19 are not found in mammals, subunits Atp20 (subunit g) and Atp21 (subunit e) are present (Kucharczyk et al., 2009). However, a role of these subunits in dimerization has not been demonstrated. Thus, information is still lacking on what mediates $\mathrm{F}_{1} \mathrm{~F}_{\mathrm{O}} \mathrm{ATPase}$ dimer-interface interactions in higher eukaryotes.

An in silico analysis was performed in order to identify novel $\mathrm{F}_{1} \mathrm{~F}_{\mathrm{O}} \mathrm{ATPase}$ dimerization factors in higher eukaryotes (Figure 9). The bioinformatics analysis was based on searching for ORFs with protein products of similar characteristics to dimerization factors already characterized in yeast (Atp18, Atp19, Atp20, and Atp21). Most importantly, the protein should have a significant human homolog, defined by a low Expect (E) value from sequence homology searches. As illustrated in Figure 9, mitochondrial localized proteins were filtered through various parameters using the SGD database. Potential ORFs of interest would code for an uncharacterized protein with at least one transmembrane domain and a size equal to or smaller than $12 \mathrm{kDa}$. Similar to Atp20 and Atp21, the protein would contain a GxxxG motif in its transmembrane domain. Two ORFs, YJR085C and YCL057c-A complied with these conditions, both having potential human homologs based on E-values, of $3 \times 10^{-8}$ and $6 \times 10^{-6}$ respectively*. In this study, YCL057c-A (termed Mio10), as well as its potential human homolog C1orf151 (termed MINOS1), were analyzed. 
A

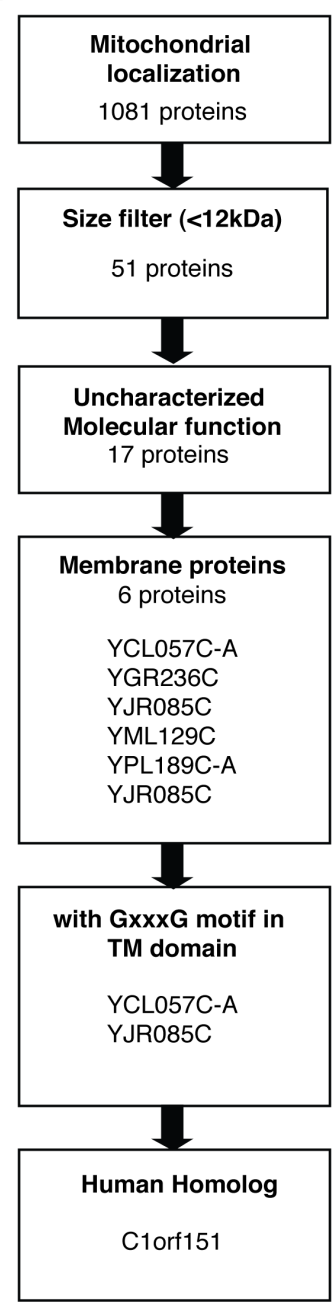

B

\begin{tabular}{|c|c|c|}
\hline $\begin{array}{l}\text { Systematic } \\
\text { name }\end{array}$ & Protein Sequence & $\begin{array}{l}\text { Potential } \\
\text { Homolog }\end{array}$ \\
\hline YCL057C-A & $\begin{array}{l}\text { MSEQAQTQQPAKSTPSKDSN } \\
\text { KNGSSVSTILDTKWDIVLSN } \\
\text { MLVKTAMGEGVGVFTSVLFE } \\
\text { KRRAFPVWLGIGEGVGRGYA } \\
\text { EGDAIFRSSAGLRSSKV }\end{array}$ & C1orf151 \\
\hline YJR085C & $\begin{array}{l}\text { MEHPAYTLSLLTTAGGLMGY } \\
\text { YRKGSIPSLVSGLVEGSVYG } \\
\text { IAGYLLHMNRDGGLEMALGA } \\
\text { STLLLGAGVIRGMPSRFTKP } \\
\text { VPVVLTALGGLGSYYYYNKY } \\
\text { KEFYP }\end{array}$ & NP_112231 \\
\hline
\end{tabular}

Figure 9. Identification of Mio10 and MINOS1 by in silico analysis. (A) The Saccharomyces Genome Database (SGD) was screened for all mitochondrial proteins with a size $\leq 12 \mathrm{kDa}$ and uncharacterized molecular function, and consequently for membrane proteins that have significant human homology (based on E values) by BLAST search. (B) Sequences of the proteins with significant human homologs. Regions with GxxxG motifs are highlighted in yellow; glycine residues are highlighted in red. Predicted TM domains are underlined. 


\subsubsection{Mio10 and MINOS1 sequence analysis and alignments}

Analysis of the primary amino acid sequences of Mio10 and MINOS1 indicated that both ORFs encode small proteins of 97 and 78 amino acids, of approximately 10 and 8 $\mathrm{kDa}$ in size, respectively (Figure 10). Sequence analysis indicated that neither has a predicted presequence. In addition, Mio10 and MINOS1 are predicted to contain two transmembrane domain each of which each contains a GxxxG motif.

A

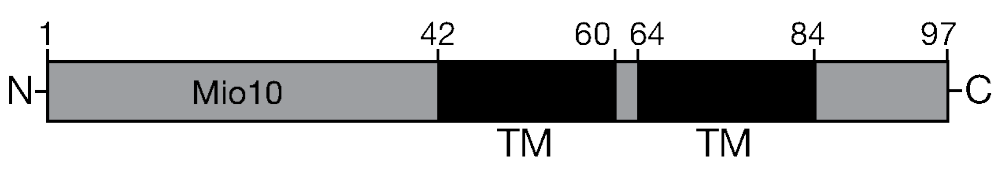

B

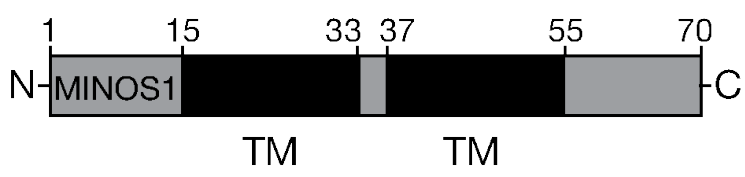

Figure 10. Molecular organization of selected proteins. (A) Mio10, and (B) MINOS1. Black boxes indicate predicted transmembrane (TM) domains. No predicted mitochondrial presequence predicted. $\mathrm{N}$, amino-terminus; $\mathrm{C}$, carboxyl-terminus.

Interestingly, Mio10 and MINOS1 appear to be highly conserved across all examined species. Figure 11 depicts a multiple sequence alignment of Mio10 in various yeast species. The $\mathrm{N}$-termini and $\mathrm{C}$-termini are weakly conserved across analyzed yeast species. Moreover, it appears that protein sequences encoding the two transmembrane domains are highly conserved with the two GxxxG motifs unaltered in all examined yeast species. The primary amino acid sequence of MINOS1 from various species representing vertebrates and invertebrates were also analyzed in a multiple sequence alignment (Figure 12). The N- and C-termini, like those of Mio10, appear to be variable across various species, whereas sequences encoding the two transmembrane domains are highly conserved, with the GxxxG motifs unaltered. This variation of $\mathrm{N}$ - and $\mathrm{C}$-termini may indicate the importance of the transmembrane domains in Mio10 and MINOS1 function. 

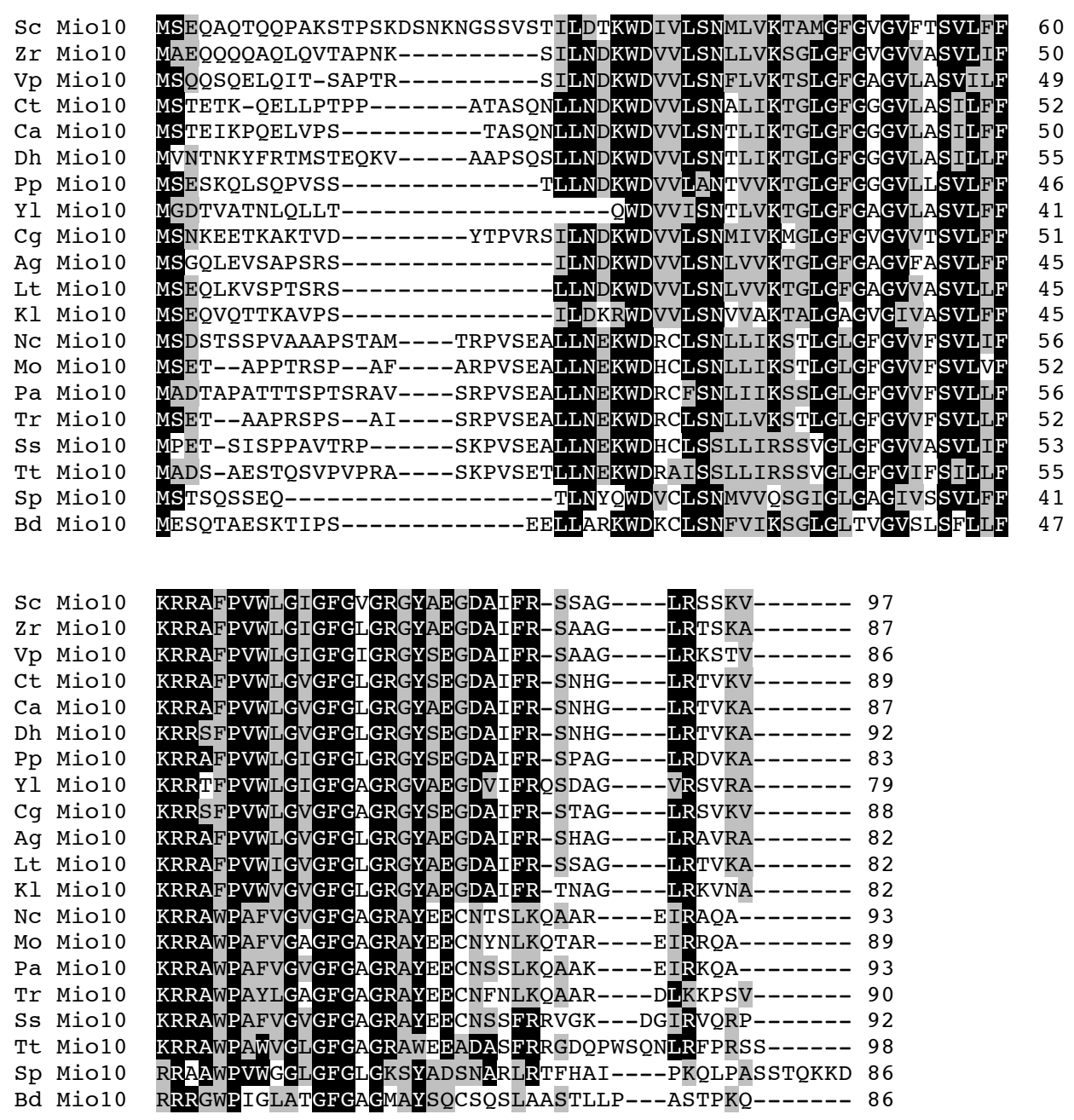

Figure 11. Alignment of Mio10 yeast homologs. Multiple sequence alignment performed by ClustalW 2.0.11. Black boxes indicate identical residues in at least four species; gray boxes indicate similar amino acids according to Erdmann et al., 1991. Sc, S. cerevisiae; Zr, Z.rouxii; Vp, V. polyspora; Ct, C. tropicalis; Ca, C. albicans; Dh, D. hansenii; Pp, P. pastoris; Y1, Y. lipolytica; Cg, C. globosum; Ag, A.gossypii; Lt, L. thermotolerans; Kl, K. lactis; Nc, N. crassa; Mo, M. oryzae; $\mathrm{Pa}, P$. anserina; $\mathrm{Tr}, T$. reesei; Ss, S. sclerotiorum; Tt, T. tonsurans; Sp, $S$. pombe; $\mathrm{Bd}, B$. dendrobatidis. 
A

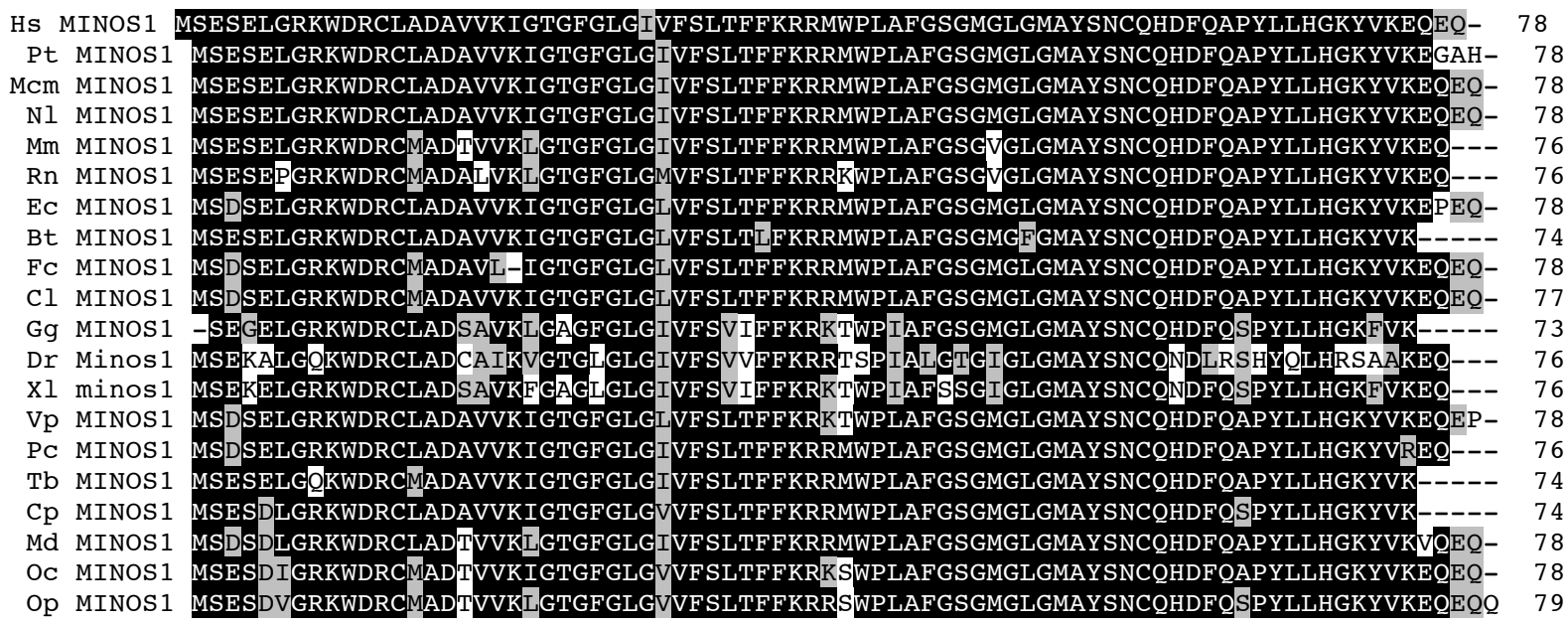

B

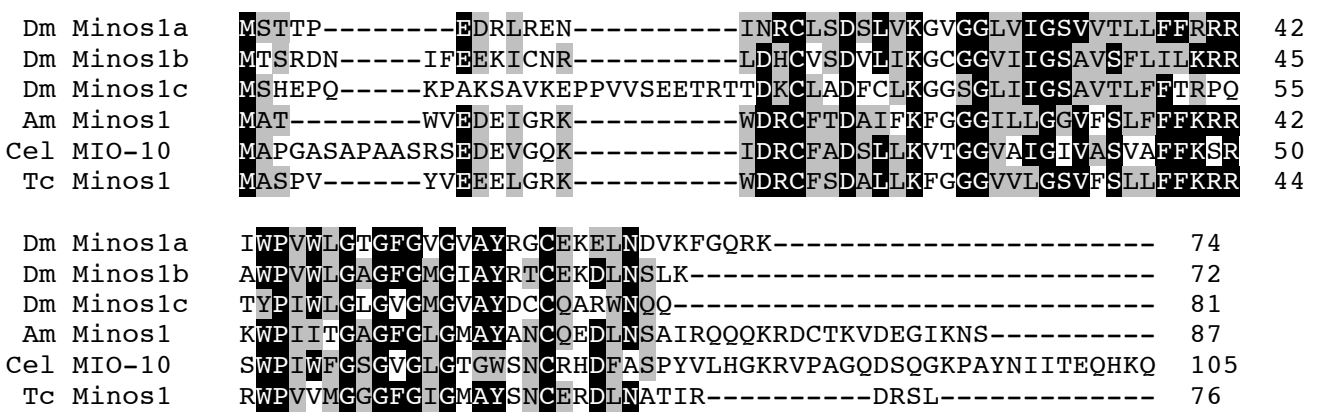

Figure 12. Alignment of MINOS1 homologs. Multiple sequences preformed by ClustalW 2.0.11. (A) Vertebrates (B) Invertebrates. Black boxes indicate identical residues in at least four species; gray boxes indicate similar amino acids according to Erdmann et al., 1991. Hs, H. sapiens; Pt, P. troglodytes; Mcm, M. mulatta; Nl, N. leucogenys; Mm, M. musculus; Rn, $R$. norvegicus; Ec, E.caballus; Bt, B. taurus; Fc, F. catus; Cl, C. lupus familiaris; Gg, G. gallus; $\mathrm{Dr}, D$. rerio; Xl, X. laevis; Vp, V. pacos; Pc, P. capensis; Tb, T. belangeri; Cp, C.porcellus; Md, M. domestica; Oc, O. cuniculus; Op, O. princeps; Dm, D. melanogaster; Am, A. mellifera; Cel, C. elegans; Tb, T. castaneum; At, A. thaliana; Gm, G. max; Os, O. sativa; Zm, Z. mays. For Dm: a, b, and c indicate different isoforms. For Cel, MINOS1 termed MIO-10 in accordance with C.elegans nomenclature guidelines. 


\subsection{Topological analysis of Mio10 and MINOS1}

$\mathrm{F}_{1} \mathrm{~F}_{\mathrm{O}} \mathrm{ATPase}$ dimerization factors are membrane proteins that interact with the inner membrane embedded $\mathrm{F}_{\mathrm{O}}$ portion of $\mathrm{F}_{1} \mathrm{~F}_{\mathrm{O}} \mathrm{ATPase}$ (Wagner et al., 2010). In order to investigate a possible role of Mio10 and MINOS1 in $\mathrm{F}_{1} \mathrm{~F}_{\mathrm{O}}$ ATPase dimerization, their association with the inner membrane was investigated. To this end, antibodies that specifically recognize Mio10 and MINOS1 were required. Once these antibodies were verified, mitochondrial sublocalization and membrane association assays were performed.

\subsubsection{Antibody generation and specificity}

To analyze endogenous MINOS1 protein signals a commercially purchased antibody against MINOS1 was used. MINOS1 antibody specificity was tested, in which a protein extract from either whole-cell lysate or from isolated mitochondria were analyzed by Western blotting (Figure 13A). In both cases, the MINOS1 antibody recognizes a protein of approximately $10 \mathrm{kDa}$ in size. The antibody non-specifically recognizes proteins of 14 and $60 \mathrm{kDa}$ in size; these proteins however are not present in the mitochondrial protein extract indicating that the only the $10 \mathrm{kDa}$ protein is imported and incorporated into mitochondria.
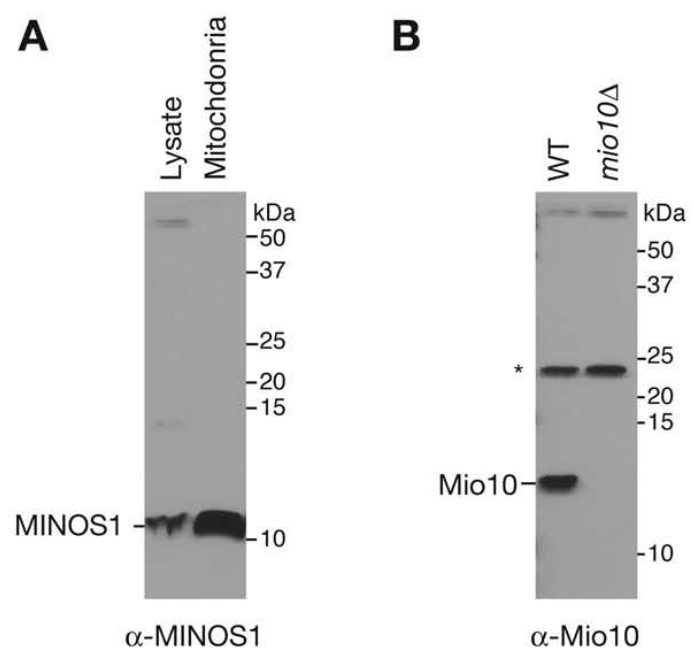

Figure 13. Antibody specificity test. (A) Western-blot analysis of HEK293T whole-cell extract (lysate) and HEK293T isolated mitochondria using MINOS1 specific antibodies. (B) Western-blot analysis of whole-cell protein extracts from wildtype and mio $10 \Delta$ yeast culture using Mio10 specific antibodies. In (A) and (B), $10 \mu \mathrm{g}$ protein was loaded on each lane. * indicates non-specific antibody cross-reaction. 
In contrast, an antibody was generated to analyze endogenous Mio10 protein signals by Western blot analysis (Figure 13B). This has been achieved by using a chemically synthesized peptide (EGDAIFRSSAGLRSSKV) corresponding to the Cterminal of the Mio10 protein. Antibody specificity evaluation was performed by analyzing whole-cell proteins extracts from wildtype and mio10 0 strains. Western blot analysis indicates that the raised antibody specifically recognizes a protein product slightly larger than $10 \mathrm{kDa}$. The antibody, however, also recognizes two nonspecific protein products of approximately 22 and $60 \mathrm{kDa}$ in size. It should be noted that the antibodies against Mio10 and MINOS1 did not recognize their respective epitopes on Blue native PAGE (BN-PAGE), which can be used to characterize large complexes in their native state (Nijtmans et al., 2002). For immunodetection purposes, the antibodies were primarily used with SDS-PAGE.

\subsubsection{Topological analysis of Mio10}

To assess whether Mio10 was a membrane protein, a carbonate extraction analysis was performed on isolated wildtype mitochondria (Figure 14A). Upon treatment with carbonate buffer, followed by high-speed centrifugation, Mio10 was carbonate resistant and remained in the membrane pellet, similar to mitochondrial integral membrane proteins Tom70 and Tim21. On the other hand, the peripherally membrane associated protein Tim44 was released into the supernatant. This result indicates that Mio10 behaves like an integral membrane protein. As a control, treatment of mitochondria with Triton X-100 (TX-100) released all proteins into the supernatant.

In addition, the sublocalization of Mio10 was assessed by a protease protection assay, in which wildtype mitochondria were treated with various concentrations of Proteinase K (Figure 14B). Tom70, which is localized at the mitochondrial outer membrane, was degraded, whereas Mio10 was protected against protease degradation. Upon osmotic disruption of the outer membrane and thus generation of mitoplasts and exposing protein contents and protein domains associated with the IMS, Mio10 was rendered accessible to protease degradation. In contrast, matrix proteins (Tim44 control) were unaffected. As the antibody is specific the C-terminus of Mio10, the partial loss of antibody signal by Western blot analysis indicates that the affected Cterminus of Mio10 is located in the IMS. This is supported by the observation that the pattern of Mio10 PK degradation pattern resembles that of Tim21, an inner membrane 
protein, in which PK treatments leads to partial degradation of its IMS exposed Cterminus (Chacinska et al., 2005). These results combined with membrane association results indicate that Mio10 is an integral mitochondrial inner membrane protein with its C-terminus exposed to the IMS (Figure 17).

A

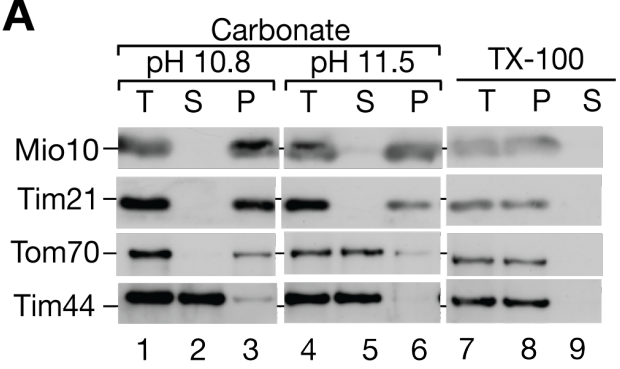

B

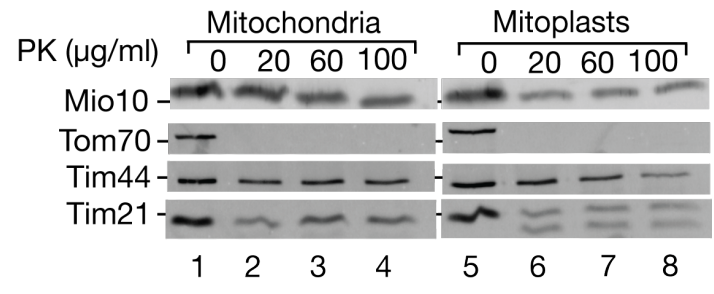

Figure 14. Mio10 is a mitochondrial IMS protein. (A) Membrane association assay. mitochondria were suspended in carbonate buffer, or alternatively lysed with $1 \%$ Triton X100. All samples were incubated on ice and then subjected to ultracentrifugation and TCA precipitation followed by SDS-PAGE and Western blot analysis. B) Protease protection assay; mitochondria, and mitoplasts were treated with various amounts of PK. The pellets of SEMtreated mitochondria and mitoplasts were resuspended in protein loading dye and analyzed by SDS-PAGE and Western blot analysis. T, total; P, pellet; S, supernatant; PK, Proteinase K; TX-100, TritonX-100.

\subsubsection{Subcellular localization of MINOS1 by immunofluorescence}

Sequence analysis of the MINOS1 ORF (C1orf151) indicated predicted a mitochondrial localization based on large-scale mitochondrial genetic screen (Baughman et al., 2009). To verify this, immunofluorescence microscopy using MINOS1 specific antibodies was performed (Figure 15). Antibodies against MINOS1 and the mitochondrial marker Cyclophilin D were applied to monkey epithelial kidney cells, VERO. Image analysis revealed co-localization of MINOS1 and Cyclophilin D antibody signals, supporting the notion that MINOS1 is a mitochondrial protein. Moreover, cells were stained with the nuclear marker DAPI, indicating that both MINOS1 and Cyclophilin D localized to mitochondrial networks. 

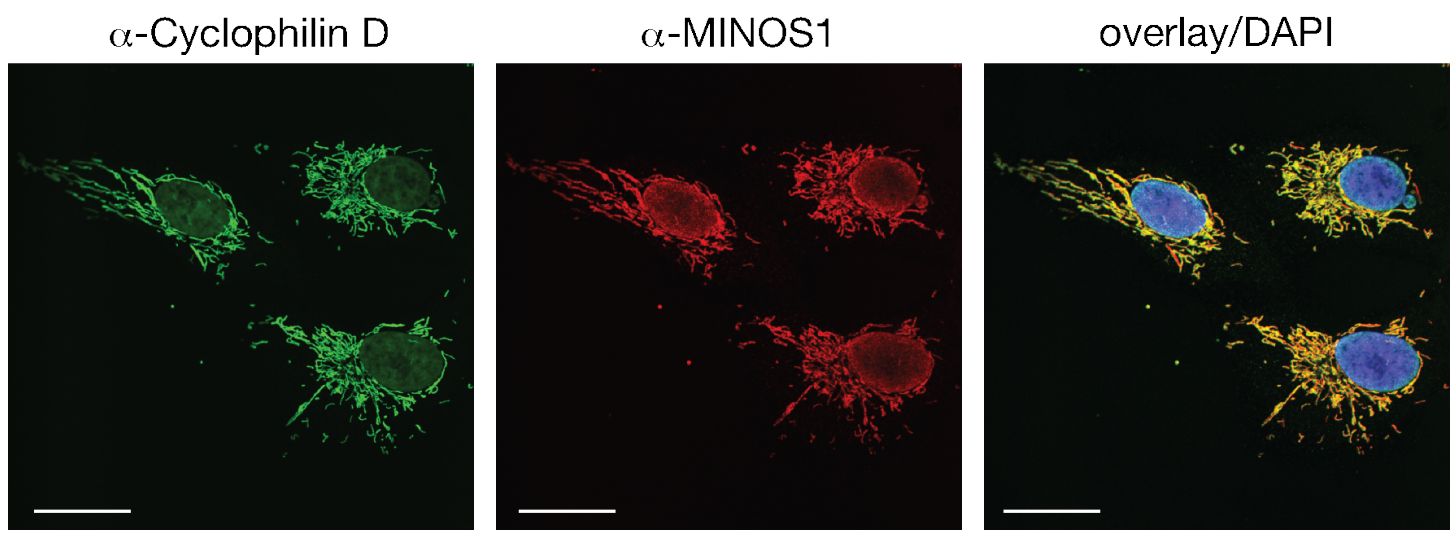

Figure 15. MINOS1 is a mitochondrial localized protein. Immunofluorescence analysis of MINOS1 localization. VERO cells were fixed in $8 \%$ paraformaldehyde (PFA) and permeabilized in $0.5 \%(\mathrm{v} / \mathrm{v})$ Triton X-100. Treated cells were then incubated for 1 hour with MINOS1-antibody and the mitochondrial marker Cyclophilin D-antibody. Cells were then washed cells and incubated with Oregon Green 488 conjugated IgGs and KK114 conjugated goat IgGs. Cells were then stained with DAPI staining before performing fluorescence microscopy. Scale bars $=10 \mu \mathrm{m}$. Microscopy was performed by Daniel Jans (AG Jakobs, MPI-bpc).

\subsubsection{Topological analysis of MINOS1}

To test MINOS1 membrane association, a carbonate extraction analysis was performed on mitochondria isolated from HEK293T cells, in which cultured cells were treated with carbonate buffer $\mathrm{pH} 10.8$ or 11.5 (Figure 16A). In all cases, MINOS1 appeared carbonate resistant and remained in the membrane pellet similar to mitochondrial integral membrane proteins TOM70 and TIM23. On the other hand, the matrix protein, TACO1, was released into the supernatant. The results thus indicate that MINOS1 is an integral membrane protein. 
A

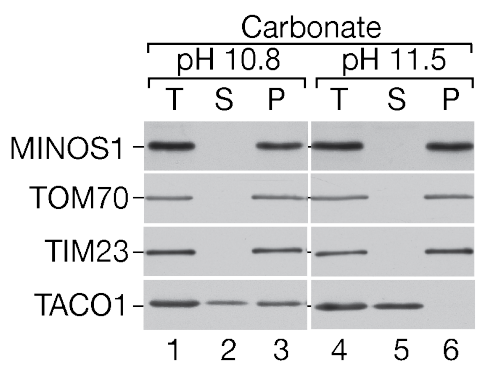

B

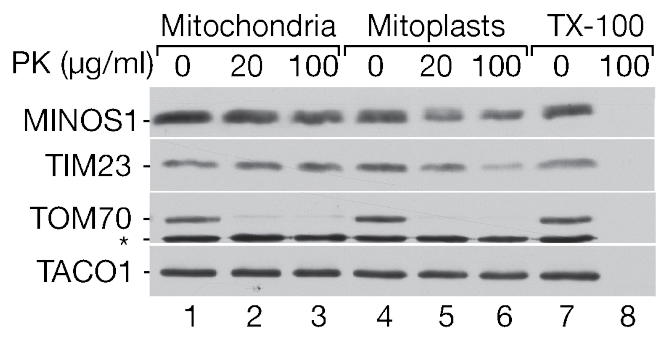

Figure 16. MINOS1 is a mitochondrial IMS protein. (A) Protease protection assay; mitochondria, mitoplasts, or Triton X-100 lysed mitochondria were treated with various amounts of PK. The pellets of SEM-treated mitochondria and mitoplasts and the supernatant of Triton X-100 lysed mitochondria were resuspended in protein loading dye and analyzed in by SDS-PAGE. PK, Proteinase K; TX-100, TritonX-100. (B) Membrane association assay. Mitochondria were suspended in carbonate buffer, or alternatively lysed with $1 \%$ Triton X100. All samples were incubated on ice and then subjected to ultracentrifugation and TCA precipitation followed by SDS-PAGE and Western blot analysis. T, total; P, pellet; S, supernatant. * indicates non-specific antibody cross-reaction.

To analyze MINOS1 sublocalization, isolated mitochondria from HEK293T cells and mitoplasts were treated with various concentrations of PK in a protease protection assay (Figure 16B). When mitochondria were treated with various concentrations of PK, MINOS1 appeared to be protected against protease degradation. TOM70, which is localized at the mitochondrial outer membrane, was degraded by PK. Moreover, when mitoplasts were treated with PK, MINOS1 was accessible to protease treatment. The matrix protein TACO1 was unaffected. MINOS1 accessibility was similar to TIM23, an inner membrane protein with its C-terminus exposed to the IMS, supporting the notion that MINOS1 has a similar topology. As a control, all proteins were degraded after treatment with T-X100. The MINOS1 pattern of PK degradation parallels that of the one observed for Mio10, and thus it was concluded that MINOS1 and Mio10 have a similar topology in the mitochondrial inner membrane. These results combined with membrane association assay results indicate that MINOS1 is an integral mitochondrial inner membrane protein with its $\mathrm{N}$ - and $\mathrm{C}$ termini exposed to IMS. Thus based on results from the protease protection assay and membrane association assay, it is deduced that the MINOS1, together with its yeast homolog Mio10, are mitochondrial inner membrane proteins with both of their termini exposed to the IMS (Figure 17). 


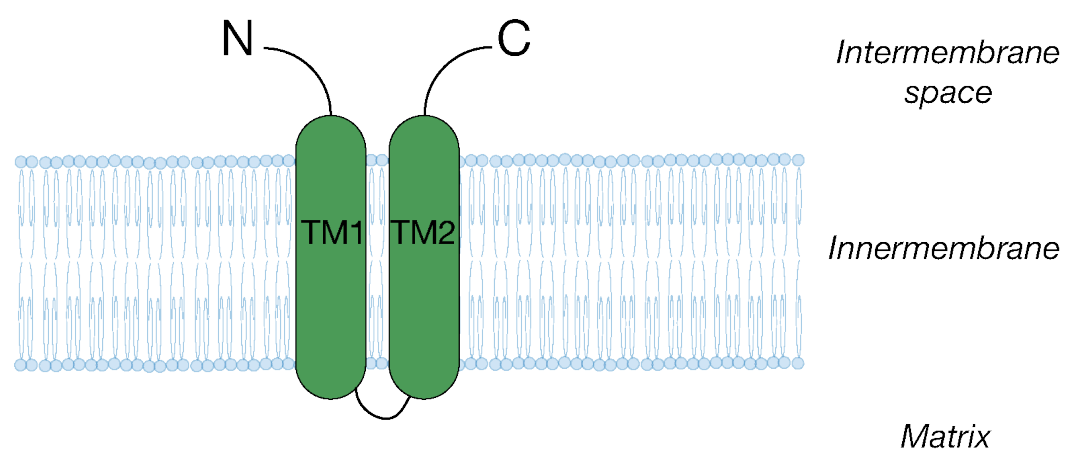

Figure 17. Topology of Mio10 and MINOS1. Subfractionation and membrane association analysis indicate that Mio10 is an integral mitochondrial inner membrane protein with its $\mathrm{N}$ and $\mathrm{C}$ termini exposed to the IMS. N, Amino-terminus; C, Carboxyl-terminus; TM, transmembrane domain.

\subsection{Analysis of Mio10 and MINOS1 association with the $F_{1} F_{0} A T P a s e$}

In order to investigate the roles of Mio10 and MINOS1 with respect to $\mathrm{F}_{1} \mathrm{~F}_{\mathrm{O}} \mathrm{ATPase}$ dimerization, Mio10 and MINOS1 containing complexes were first investigated by BN-PAGE. This technique allows the separation of large proteins complexes without their dissociation and has been useful in the analysis of various $F_{1} F_{O} A T P a s e$ dimerization factors (Arnold et al., 1999; Nijtmans et al., 2002; Wagner et al., 2009). Because of the Mio10 and MINOS1 antibodies limitations on BN-PAGE, in vitro synthesized radiolabeled precursors of the proteins were used. Import and assembly were performed. In addition, interactions of Mio10 and MINOS1 with $\mathrm{F}_{1} \mathrm{~F}_{\mathrm{O}}$ ATPase were investigated by biochemical isolations from strains containing tagged $\mathrm{F}_{1} \mathrm{~F}_{\mathrm{O}} \mathrm{ATPase}$ components.

\subsection{1 in vitro import of Mio10 and analysis by SDS-PAGE}

$\mathrm{A}{ }^{35}$ S-labeled Mio10 precursor was synthesized in vitro and imported into energized or membrane potential depleted wildtype mitochondria in the presence or absence of PK (Figure 18). Mio10 is imported in a non-membrane potential manner. Furthermore, Mio10 appears to be protected against PK treatment. As a control $\left[{ }^{35} \mathrm{~S}\right] \mathrm{Su}$ 9-DHFR was imported. This preprotein is composed of the N-terminal 69 residues of the $\mathrm{F}_{1} \mathrm{~F}_{\mathrm{O}} \mathrm{ATPase}$ subunit 9 (Su9) precursor of N.crassa, fused with the mouse dihydrofolate reductase (DHFR) (Pfanner et al., 1987). In contrast to Mio10 import, Su9-DHFR import indicated both membrane potential dependency and PK sensitivity. 
A

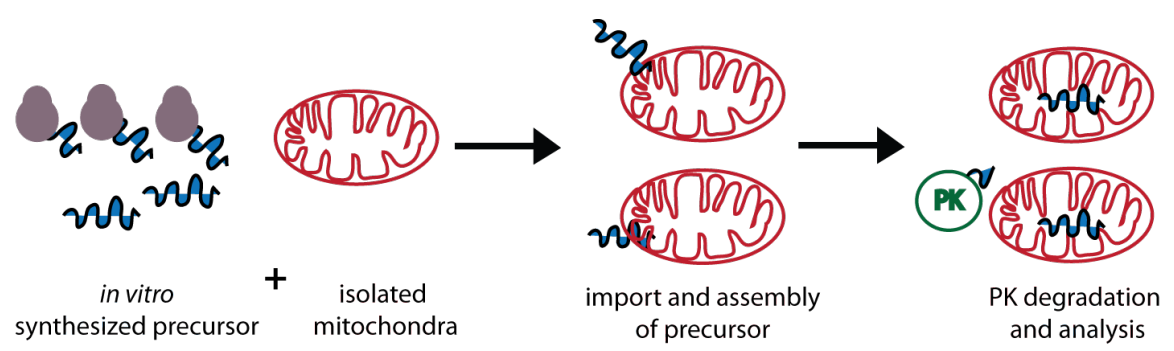

B

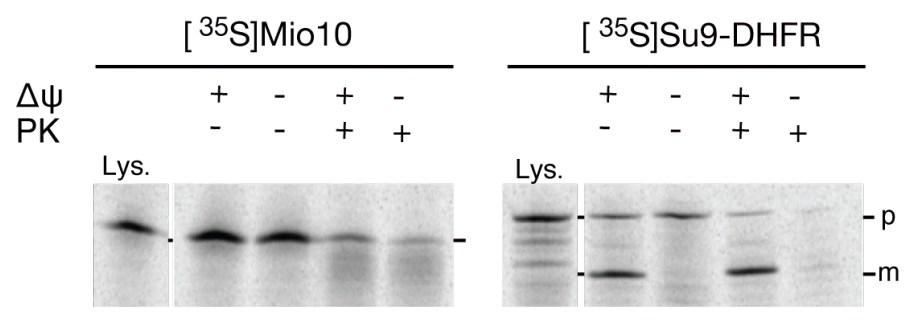

Figure 18. Import of Mio10 into mitochondria is membrane potential independent. (A) Schematic diagram representing in vitro import of radiolabeled lysate in mitochondria. (B) Radiolabeled Mio10 and Su9-DHFR were imported into isolated mitochondria in the presence or absence of a membrane potential for the indicated times. Samples were split and left untreated or treated with proteinase K (PK) and analyzed by Tricine-SDS-PAGE and digital autoradiography. As control, lysate containing Mio10 precursor or Su9-DHFR were applied (Lys.); p, precursor; $\mathrm{m}$, mature protein.

\subsubsection{Mio10 and MINOS1 assembly analysis by BN-PAGE}

Radiolabeled Mio10 and MINOS1 labeled precursors were synthesized in vitro and imported into energized or membrane potential depleted wildtype yeast mitochondria, which were then solubilized in mild digitonin buffer and analyzed by BN-PAGE (Figure 19A). Mio10 assembles in a time and membrane potential dependent manner into complexes of various size, ranging from high molecular components ( $>669 \mathrm{kDa})$ to smaller complexes (approximately $160 \mathrm{kDa}$ ). Interestingly, MINOS1 imports and assembles in yeast mitochondria. Moreover Mio10 and MINOS1 labeled precursors were imported and assembled in HEK293T isolated mitochondria (Figure 19B). Mio10 appears to assemble in human mitochondria, at which Mio10 and also MINOS1, as in yeast mitochondria, form a range of complexes. 
A

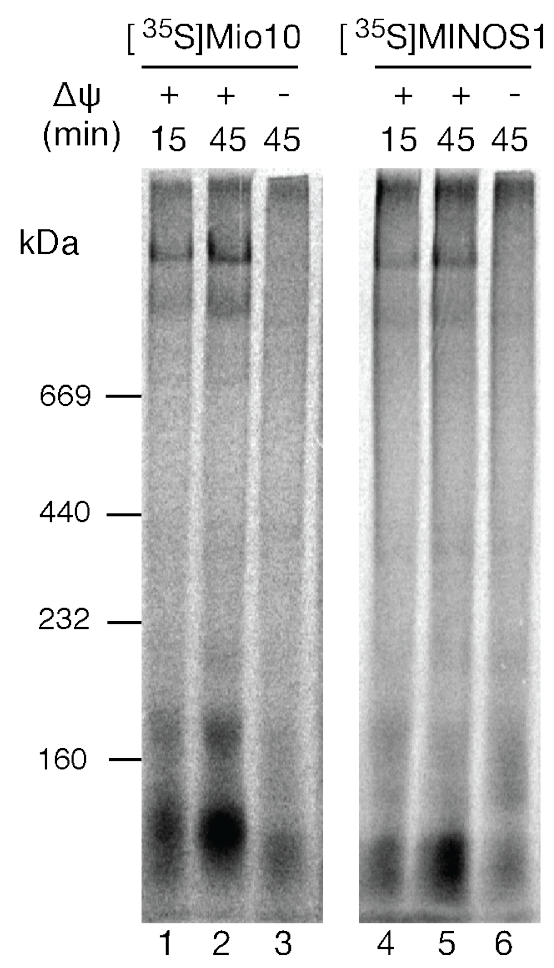

B

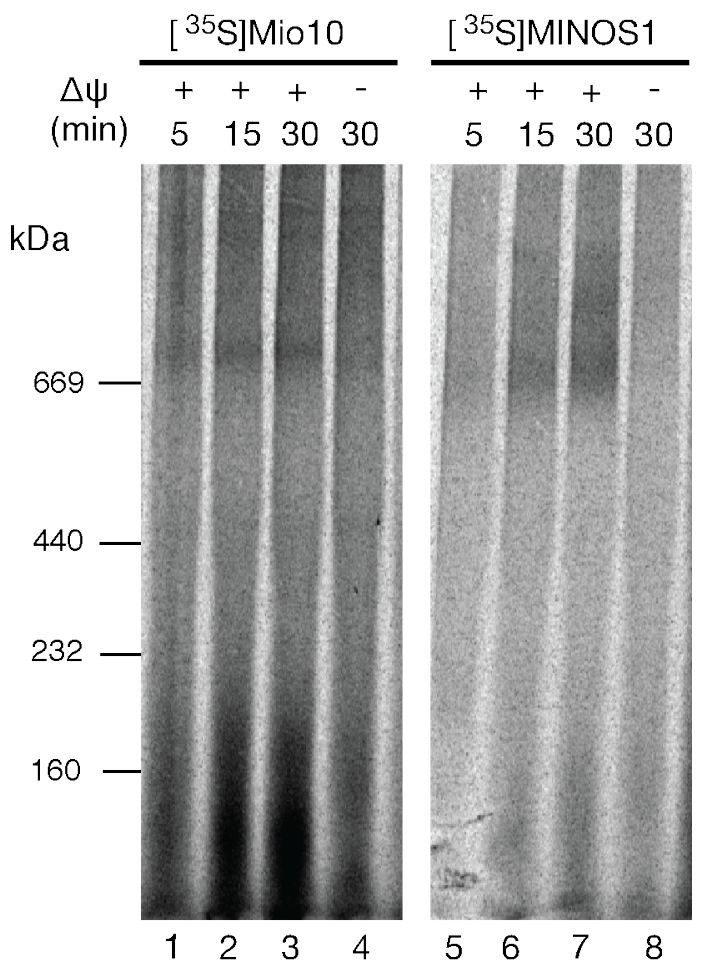

Figure 19. Mio10 and MINOS1 assemble into high molecular sized mitochondrial

complexes. Radiolabeled Mio10 precursor was imported into isolated wildtype mitochondria in the presence or absence of membrane potential for the indicated times. Radiolabeled Atp21, Atp20 and Cox13 were imported in parallel. All samples were treated with proteinase K, solubilized in digitonin buffer, and subjected to BN-PAGE (4-10\% gradient gel). Protein complexes signals were detected by digital autoradiography. $\mathrm{V}_{2}, \mathrm{~V}, \mathrm{III}_{2} \mathrm{IV}$, and $\mathrm{III}_{2} \mathrm{IV}_{2}$ indicate respiratory chain complexes.

\subsubsection{Comparison of Mio10 complexes assembly with the $F_{1} F_{0} A T P a s e$}

In order to analyze the biogenesis of Mio10-containing complexes in mitochondria a radiolabeled precursor of Mio10 was imported into wildtype isolated mitochondria and then analyzed by BN-PAGE (Figure 20). In comparison, Cox13, a structural subunit of the cytochrome $c$ oxidase, was assembled in wildtype mitochondria (Tsukihara et al., 1996). Indeed, Mio10 assembles into complexes larger than respiratory chain supercomplexes, $\mathrm{III}_{2} \mathrm{IV}$ and $\mathrm{III}_{2} \mathrm{IV}_{2}$. When Atp20 assembly was preformed in comparison, Mio10 higher complexes are observed to migrate at a similar molecular size of the $\mathrm{F}_{1} \mathrm{~F}_{\mathrm{O}}$-ATPsynthase monomer and dimers. Moreover, it appears that Mio10 may associate with the $\mathrm{F}_{1} \mathrm{~F}_{\mathrm{O}} \mathrm{ATPase}$ dimer $\left(\mathrm{V}_{2}\right)$ with a lesser extent to the monomer (V) and also what appears to be an intermediate complex. 


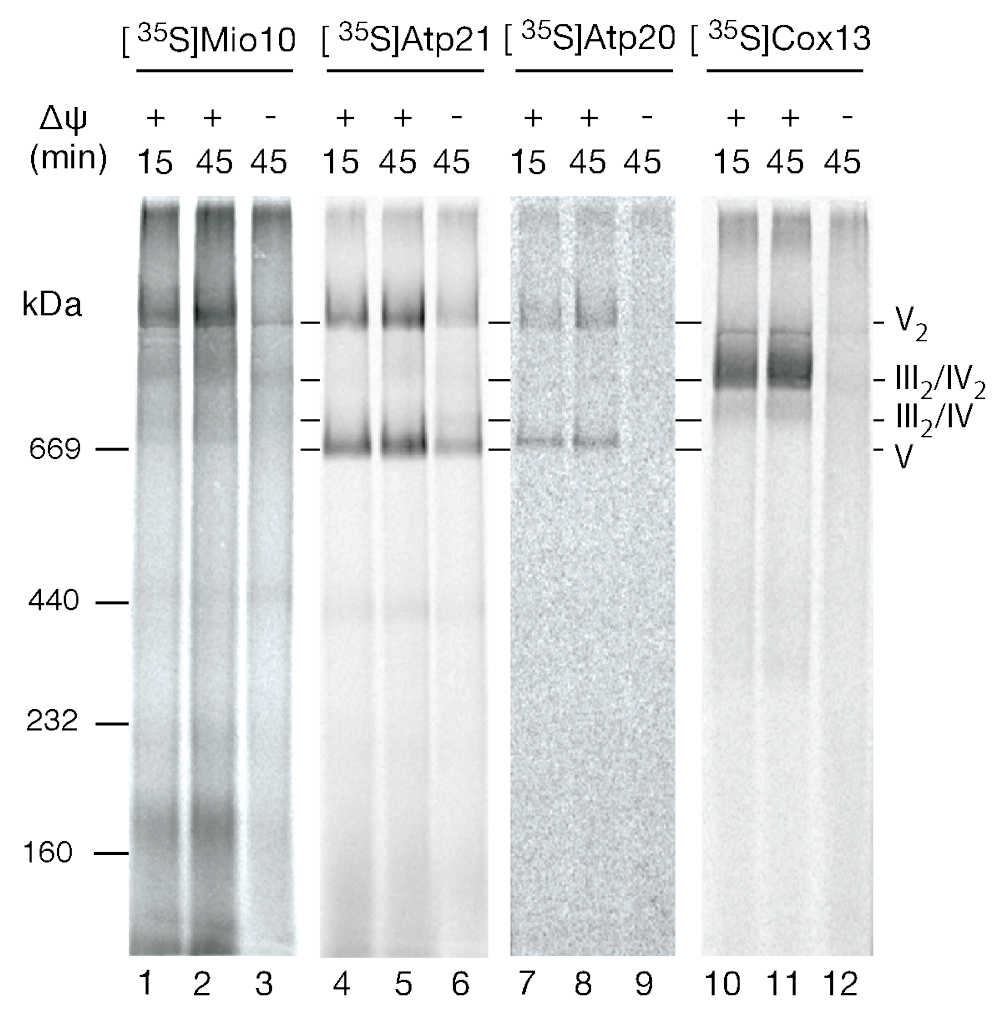

Figure 20. Mio10 comigrates with $\mathbf{F}_{1} \mathbf{F}_{\mathbf{O}} \mathbf{A T P a s e}$ complexes. Radiolabeled Mio10 precursor was imported into isolated wildtype mitochondria in the presence or absence of membrane potential for the indicated times. Radiolabeled Atp21, Atp20 and Cox13 were also imported in parallel. All Samples were treated with proteinase K, solubilized in digitonin buffer, before complex separation by BN-PAGE (4-10\% gradient gel). Protein complexes signals were detected by digital autoradiography. $\mathrm{V}_{2}$. V, $\mathrm{III}_{2} \mathrm{IV}, \mathrm{III}_{2} \mathrm{IV}_{2}$ indicate respiratory chain complexes. 


\subsubsection{Analysis of Mio10 assembly in the absence of $F_{1} F_{O} A T P a s e$ dimers}

To verify Mio10 association with the $\mathrm{F}_{1} \mathrm{~F}_{\mathrm{O}} \mathrm{ATPase}$, a Mio10 radiolabeled precursor protein was imported into atp $20 \Delta$ mitochondria, which lack an assembled $\mathrm{F}_{1} \mathrm{~F}_{\mathrm{O}} \mathrm{ATPase}$ dimer due to the absence of the dimerization factor, Atp20 (Figure 21A). As a control,

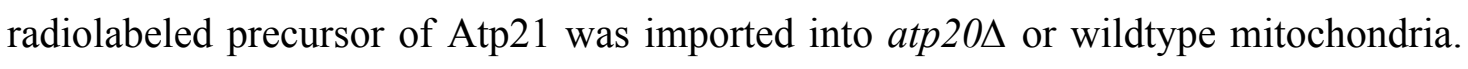
As expected, Atp2 1 assembles into the $\mathrm{F}_{1} \mathrm{~F}_{\mathrm{O}} \mathrm{ATPase}$ dimeric and monomeric forms in wildtype mitochondria whereas in atp20د mitochondria, it exclusively assembles to the $\mathrm{F}_{1} \mathrm{~F}_{\mathrm{O}} \mathrm{ATPase}$ monomer (Wagner et al., 2009). In parallel, Mio10 does not assemble

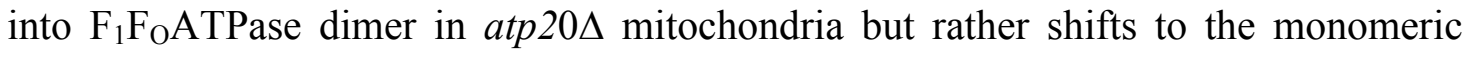
form; supporting the notion that Mio10 may be an $\mathrm{F}_{1} \mathrm{~F}_{\mathrm{O}} \mathrm{ATPase}$ associated protein. Furthermore, analysis by BN-PAGE suggests the requirement of Atp20 for proper of Mio10 assembly.

In order to rule out the possibility of post lysis association of Mio10 assembly into the $\mathrm{F}_{1} \mathrm{~F}_{\mathrm{O}} \mathrm{ATPase}$, a 'Mix' assembly assay was performed (Figure 21B). Mio10 assembly was carried out as above into atp200 mitochondria and into wildtype mitochondria without addition of radiolabeled lysate (Mock); reactions were mixed before analysis by BN-PAGE (Figure 21C). If Mio10 association with the $\mathrm{F}_{1} \mathrm{~F}_{\mathrm{O}} \mathrm{ATPase}$ were non-specific, then mixing the assembly reactions would give a positive signal corresponding to the dimer. As a control, Mio10 assembly in wildtype background was performed in juxtaposition. Results however indicated that the Mio10 large complexes continue to associate with $\mathrm{F}_{1} \mathrm{~F}_{\mathrm{O}} \mathrm{ATPase}$ dimer ruling out possible non-specific association. 
A

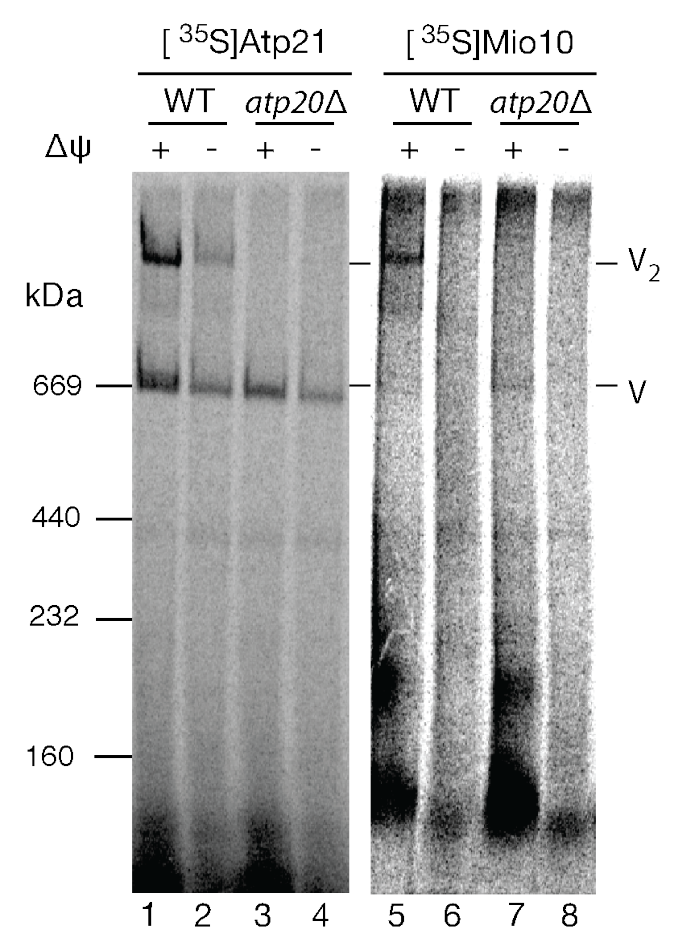

C

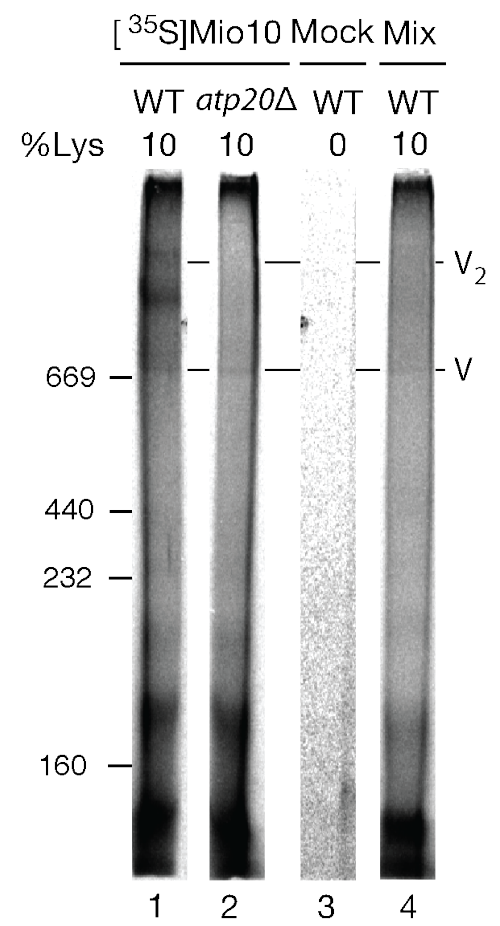

B

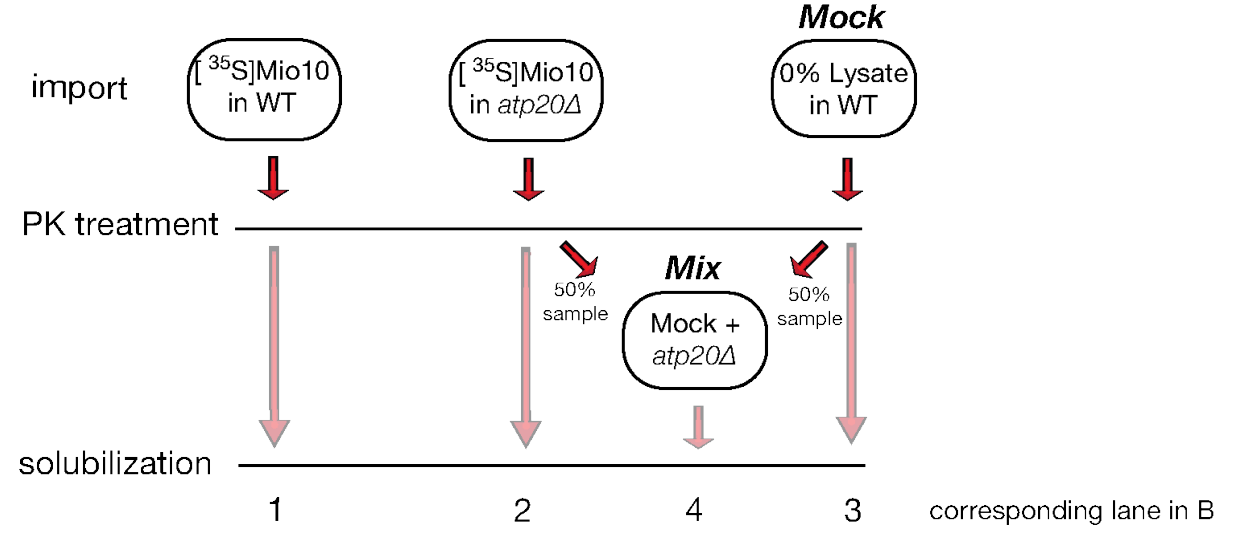

Figure 21. Mio10 assembly requires the presence of Atp20. (A) Radiolabeled Atp21 and Mio10 precursor were imported into isolated wildtype and atp204 mitochondria in the presence or absence of membrane potential for the indicated times. (B) Schematic diagram describing 'Mix' assembly assay. (C) Mix assembly assay. Assembly was performed in WT and in atp20 $\Delta$. A mock assay performed with no lysate added. After import, mitochondria were treated with PK. 50\% of the atp20 $\Delta$ assay sample was mixed with $50 \%$ of the mock. Lane 3 overexposed for clarity. (A, C) All Samples were treated with proteinase $\mathrm{K}$, solubilized in digitonin buffer, before complex separation by $\mathrm{BN}$ PAGE (4-10\% gradient gel). Protein complexes signals were detected by digital autoradiography. $\mathrm{V}_{2}, \mathrm{~V}, \mathrm{III}_{2} \mathrm{IV}, \mathrm{III}_{2} \mathrm{IV}_{2}$ : respiratory chain complexes. 


\subsubsection{Second dimension analysis of Mio10 complexes in atp20 $\Delta$ mitochondria}

Import and assembly of radiolabeled Mio10 indicated that Mio10 associates with what appears to be the $\mathrm{F}_{1} \mathrm{~F}_{\mathrm{O}} \mathrm{ATPase}$ dimer in wildtype mitochondria, whereas this

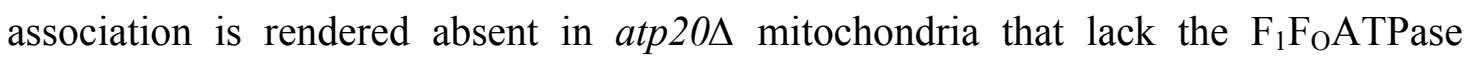
dimer. To investigate this observation further, a second dimension analysis of atp20د

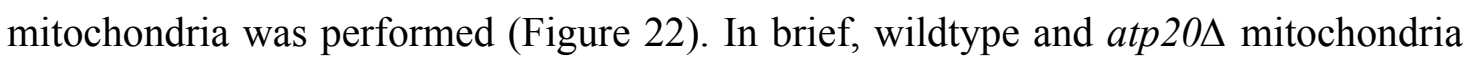
were solubilized in digitonin buffer followed by separation of protein complexes on BN-PAGE. Individual lanes from the $\mathrm{BN}$ gel were sliced and further subjected to SDS-PAGE analysis (second dimension). The aim of the approach was to examine Mio10 signals in comparison to the $\mathrm{F}_{1} \mathrm{~F}_{\mathrm{O}} \mathrm{ATPase}$ markers on second dimension as Mio10 antibodies did not recognize native Mio10 epitopes on BN-PAGE.

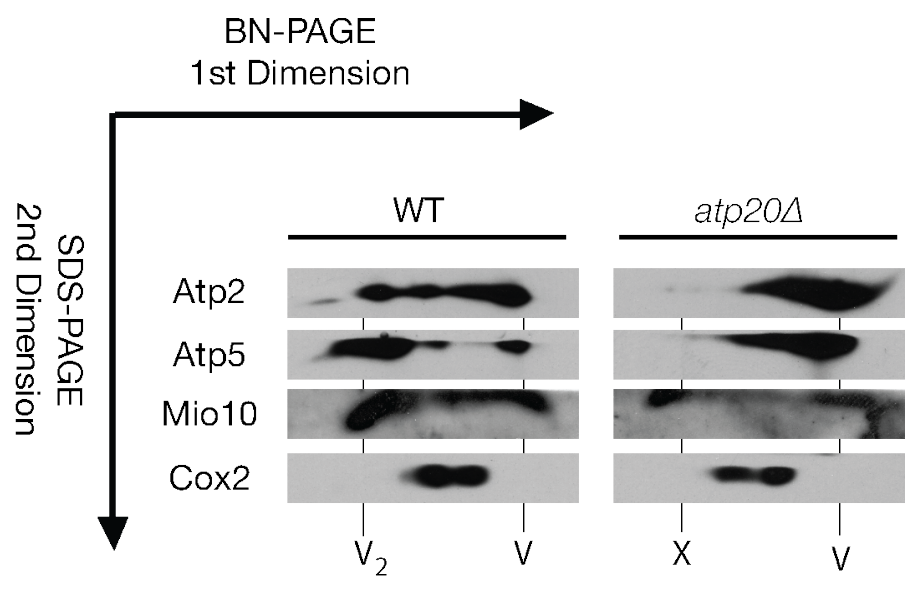

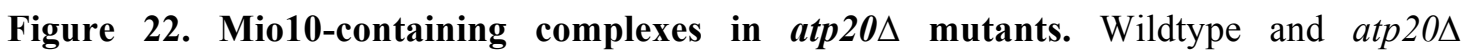
mitochondria were solubilized in digitonin buffer, subjected to BN-PAGE (4-10\% gradient gel). BN gel stripes were excised and subjected to SDS-PAGE. Signals were detected by Western blotting, $\mathrm{V}_{2}$, dimer; $\mathrm{V}$, monomer; $\mathrm{X}$, undefined.

Immunoblotting for Atp2 or Atp5 enables detection of $\mathrm{F}_{1} \mathrm{~F}_{\mathrm{O}} \mathrm{ATPase}$ monomers and dimers and thus a tool to assess status of dimerization. In wildtype mitochondria, Atp2 or Atp5 immunoblots indicate two large distinct points corresponding to the $\mathrm{F}_{1} \mathrm{~F}_{\mathrm{O}} \mathrm{ATPase}$ monomer and dimer. In addition, Cox 2 detection was used as a negative control and signals were unchanged in both wildtype and atp20 mitochondria. In atp20 mitochondria, Atp2 and Atp5 immunodetection yielded only on distinct signal corresponding to the $\mathrm{F}_{1} \mathrm{~F}_{\mathrm{O}} \mathrm{ATPase}$ monomer. Moreover, checking for Mio10 in the wildtype indicates two distinct points similar to the Atp5 and Atp2, in agreement with the import and assembly results. However, the two Mio10 specific signals remain 
present in atp20د mitochondria, indicating that the Mio10 high molecular sized complex (initially argued to be the $\mathrm{F}_{1} \mathrm{~F}_{\mathrm{O}} \mathrm{ATPase}$ dimer and labeled ' $\mathrm{X}$ ' in Figure 22) continues to appear even in the absence of properly assembled $F_{1} F_{O} A T P a s e$ dimer. Hence, second dimension analysis argues against Mio10 assembly and/or association with the $\mathrm{F}_{1} \mathrm{~F}_{\mathrm{O}} \mathrm{ATPase}$ dimer as shown in the import and assembly assays. The Mio10containing complex may thus have similar molecular sizes to the $\mathrm{F}_{1} \mathrm{~F}_{\mathrm{O}} \mathrm{ATPase}$ and comigrate in a similar fashion in BN-PAGE.

\subsubsection{Biochemical isolation of $F_{1} F_{0} A T P a s e$ components}

In a further attempt to demonstrate Mio10 $\mathrm{F}_{1} \mathrm{~F}_{\mathrm{O}}$ ATPase association, biochemical isolations from an Atp20 ${ }^{\mathrm{zz}}$ strain (in which ProteinA was C-terminally fused to Atp20) were performed (Figure 23A). Immunoprecipitation was successful in co-isolating $\mathrm{F}_{1} \mathrm{~F}_{\mathrm{O}} \mathrm{ATPase}$ subunits such as Atp5 and the Atp20 ${ }^{\mathrm{zz}}$ bait. However, Mio10 was not coisolated with Atp20. Moreover, decorations for Cox2 (cytochrome $c$ oxidase), Rip1 (complex III) or Tom70 (TOM) were negative. All together, immunoprecipitation indicate that in disagreement with initial assembly results, Mio10 is not stably associated with the $\mathrm{F}_{1} \mathrm{~F}_{\mathrm{O}} \mathrm{ATPase}$.

A

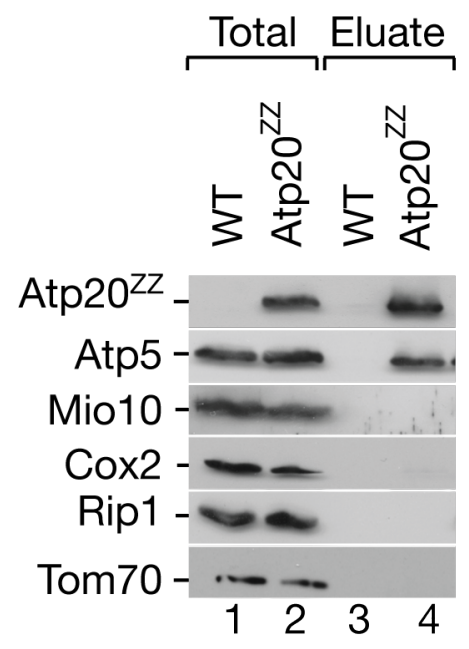

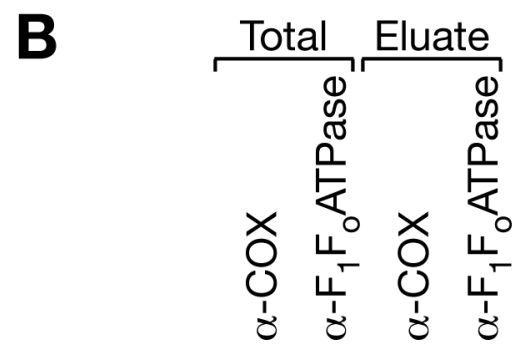

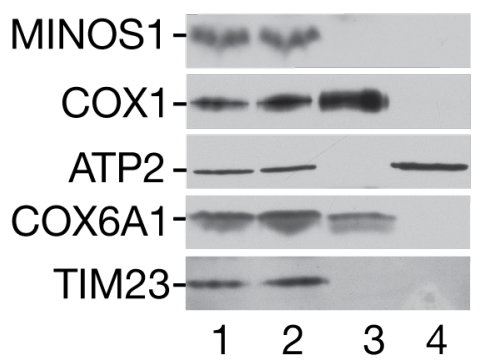

Figure 23. Mio10 and MINOS1 are not stably associated with the $F_{1} F_{0} A T P a s e$. (A) Wildtype and Atp20 $0^{\mathrm{zz}}$ mitochondria were solubilized and subjected to IgG- chromatography. Eluates were analyzed by SDS-PAGE and Western blotting. (B) Co-immunoprecipitations of cytochrome $c$ oxidase $(\mathrm{COX})$ and $\mathrm{F}_{1} \mathrm{~F}_{\mathrm{O}}$ ATPase components from isolated HEK29T mitochondria. Eluates were analyzed by SDS-PAGE and Western blotting. 
To investigate whether MINOS1 associates with the $\mathrm{F}_{1} \mathrm{~F}_{\mathrm{O}} \mathrm{ATPa}$ (1) higher eukaryotes, co-immunoprecipitations were performed from HEK293T mitochondria. Commercial antibodies specific to either the $\mathrm{F}_{1} \mathrm{~F}_{\mathrm{O}}$ ATPase $\left(\alpha-\mathrm{F}_{1} \mathrm{~F}_{\mathrm{O}}\right.$ ATPase $)$ or $(\alpha-$ $\mathrm{COX}$ ) as a negative control were used (Figure 23B). $\alpha$-COX efficiently immunoprecipitates cytochrome $c$ oxidase subunits COX1 and COX6A1 but not MINOS1, ATP2, or TIM23. In contrast, $\left(\alpha-\mathrm{F}_{1} \mathrm{~F}_{\mathrm{O}} \mathrm{ATPase}\right)$ antibodies successfully immunoprecipitated ATP2; but not MINOS1 or any of the negative controls. The results thus indicate that, as shown with Mio10 in yeast mitochondria, MINOS1 is also not stably associated with the $\mathrm{F}_{1} \mathrm{~F}_{\mathrm{O}} \mathrm{ATPase}$.

\subsection{MIO10 deletion mutant analysis}

As biochemical isolations indicated that Mio10 and MINOS1 do not associate with the $\mathrm{F}_{1} \mathrm{~F}_{\mathrm{O}} \mathrm{ATPase}$, a possible function in mitochondrial respiration and $\mathrm{F}_{1} \mathrm{~F}_{\mathrm{O}} \mathrm{ATPase}$ activity was investigated. To this end, a MIO10 deletion strain (mio10D), in which the YCL057c-A ORF was replaced by a kanamycin cassette (OpenBiosystems), was used. Any effect that the absence of Mio10 may have on $\mathrm{F}_{1} \mathrm{~F}_{\mathrm{O}}$ ATPase dimerization and oligomerization was also investigated.

\subsubsection{Growth analysis of $M I O 10$ deletion mutant mitochondria}

To determine the effect of Mio10 on mitochondrial respiration, a growth behavior analysis was performed. Wildtype and miol0s cells were grown on fermentable (glucose) and non-fermentable (glycerol) medium plates at $30^{\circ} \mathrm{C}$ (Figure $24 \mathrm{~A}$ ). In addition, the non-respiring atp $2 \Delta$ strain as well as atp20 mutants were used for comparison. A failure to grow on fermentable media may indicate respiratory chain function as shown for various respiratory chain mutants (Mick et al., 2010). Indeed, all strains grow as expected on glucose, meanwhile, the atp $2 \Delta$ mutant, which lacks a functional $\mathrm{F}_{1} \mathrm{~F}_{\mathrm{O}} \mathrm{ATPase}$, fails to grow. The deletion of dimerization factors does not affect respiration, as the $\mathrm{F}_{1} \mathrm{~F}_{\mathrm{O}} \mathrm{ATPase}$ monomers are still functionally active (Wittig

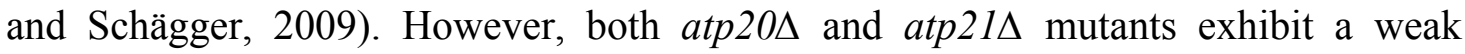
phenotype when grown on non-fermentable medium (Arnold et al., 1998).

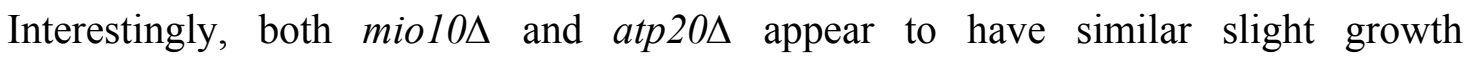
impediments compared to wildtype and atp $2 \Delta$ mitochondria. The results from the growth behavior analysis indicate that Mio10 may not be required for respiration. 


\subsubsection{Analysis of respiratory chain complexes in mio10 $\Delta$}

The MIO10 deletion strain was used to further assess a Mio10 role in $\mathrm{F}_{1} \mathrm{~F}_{\mathrm{O}} \mathrm{ATPase}$ assembly. Wildtype and mio10 mutant mitochondria were analyzed by BN-PAGE and Western blot analysis (Figure 24B). Immunodetection for the $\mathrm{F}_{1} \mathrm{~F}_{\mathrm{O}} \mathrm{ATPase}$ protein,

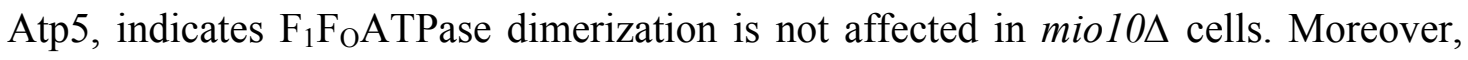
the absence of Mio10 does not affect complex III or cytochrome $c$ oxidase assembly (indicated by immunodetection with Rip1 and Cox 4 antibodies, respectively). Mio10 thus appears not to have a role in the assembly of the $\mathrm{F}_{1} \mathrm{~F}_{\mathrm{O}} \mathrm{ATPase}$ or other respiratory chain supercomplexes in yeast.

A

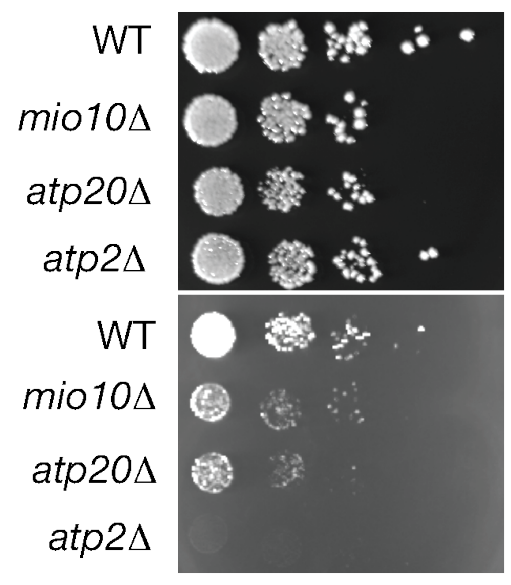

B

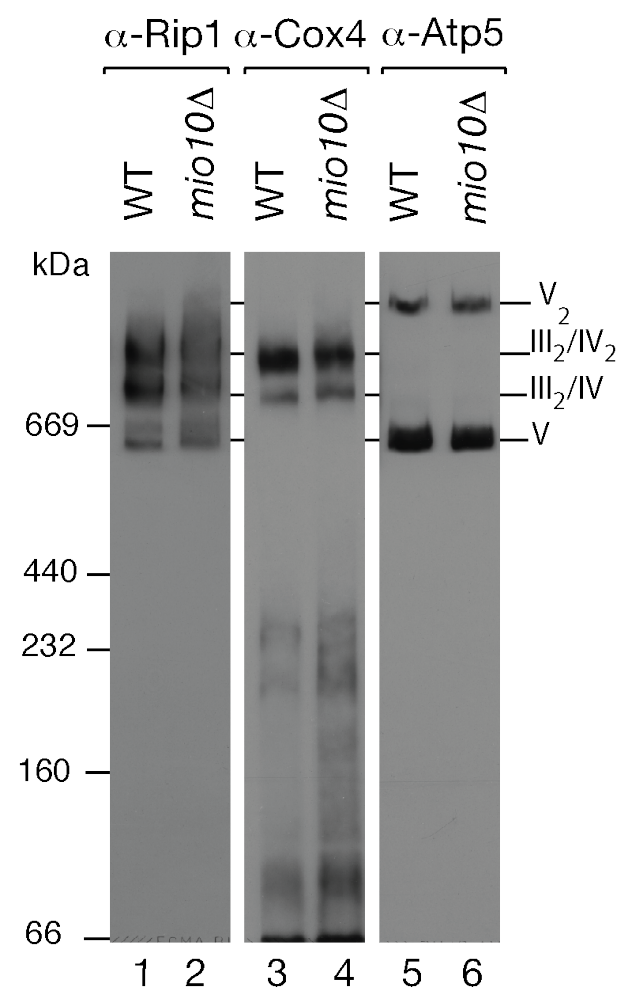

Figure 24. Mio10 is not required for mitochondrial respiration or supercomplex

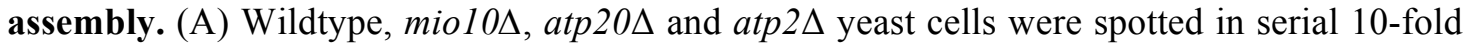
dilutions on fermentable (glucose) and non-fermentable (glycerol) media. (B) Wildtype and mio10 10 mitochondria were solubilized in digitonin buffer, subjected to BN-PAGE (4-10\% gradient gel) and Western blotting. 


\subsubsection{Assessment of $F_{1} F_{O} A T P a s e$ activity and oligomerization in mio10 $\Delta$ mutants}

Mitochondrial respiratory chain complexes retain their enzymatic activity after separation by BN-PAGE (Wittig et al., 2007). To this end, an in-gel $\mathrm{F}_{1} \mathrm{~F}_{\mathrm{O}} \mathrm{ATPase}$ activity assay was performed comparing wildtype and mio $10 \Delta$ mitochondria (Figure 25A). The in-gel activity assay (based on reverse action of the enzyme, ATP hydrolysis) indicates that $\mathrm{F}_{1} \mathrm{~F}_{\mathrm{O}} \mathrm{ATPase}$ activity is comparable in wildtype and mio $10 \Delta$ mitochondria and is unaffected by the MIO10 deletion. Furthermore, neither cytochrome $c$ oxidase nor complex I activities were significantly affected.

Given that most of the analysis performed in this study so far focused on $\mathrm{F}_{1} \mathrm{~F}_{\mathrm{O}} \mathrm{ATPase}$ dimerization, the effect on higher $\mathrm{F}_{1} \mathrm{~F}_{\mathrm{O}}$ ATPase oligomers should be not be disregarded. To this end, an in gel $\mathrm{F}_{1} \mathrm{~F}_{\mathrm{O}}$ ATPase activity assay was performed on a modified $\mathrm{BN}$ gel to better separate higher $\mathrm{F}_{1} \mathrm{~F}_{\mathrm{O}} \mathrm{ATPase}$ oligomers (Figure 25B). As a control, atp $20 \Delta$ were again used in parallel with $f c j 1 \Delta$ mitochondria. Deletion of FCJ1 augments the stability of higher $\mathrm{F}_{1} \mathrm{~F}_{\mathrm{O}} \mathrm{ATPase}$ oligomers (Rabl et al., 2009). A modified in-gel activity assay in which digitonin buffer conditions and the BN-PAGE gel gradient were altered. The assay revealed various bands corresponding to the

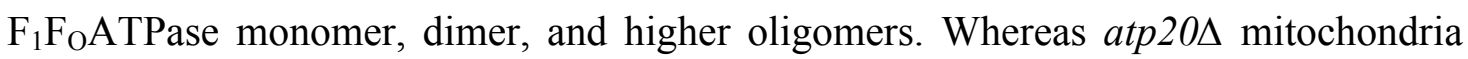
have an increased pool of $\mathrm{F}_{1} \mathrm{~F}_{\mathrm{O}} \mathrm{ATPase}$ monomer (at $669 \mathrm{kDa}$ ), there are no other apparent discrepancies between the other strains. It is thus evident, based on the in-gel activity assay, that there is no change in $\mathrm{F}_{1} \mathrm{~F}_{\mathrm{O}} \mathrm{ATPase}$ oligomerization in mio10 mitochondria compared to wildtype mitochondria. As $\mathrm{F}_{1} \mathrm{~F}_{\mathrm{O}} \mathrm{ATPase}$ activity was also not affected or did Mio10 (and MINOS1) associate with $\mathrm{F}_{1} \mathrm{~F}_{\mathrm{O}} \mathrm{ATPase}$, results indicate that neither Mio10 nor MINOS1 are $\mathrm{F}_{1} \mathrm{~F}_{\mathrm{O}}$ ATPase dimerization factors. 
A
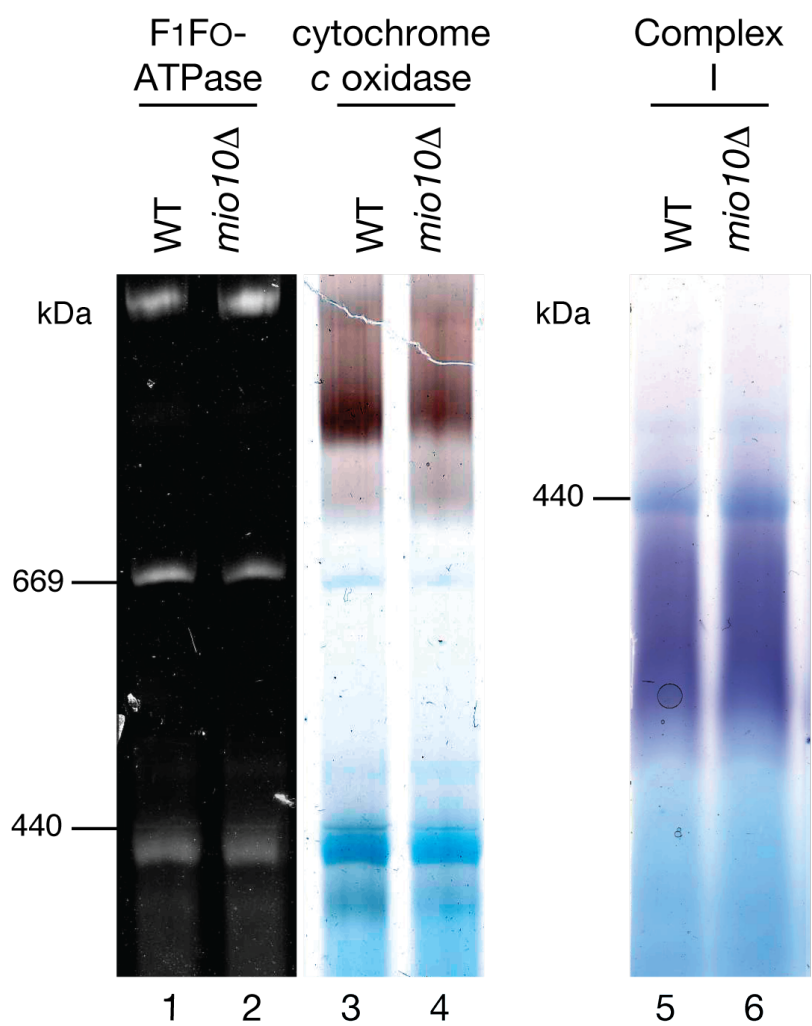

B

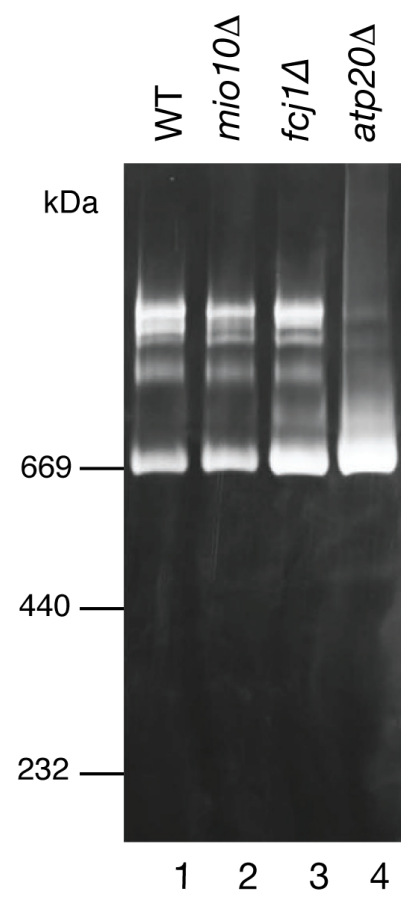

Figure 25. $\mathrm{F}_{1} \mathrm{~F}_{\mathrm{O}} \mathrm{ATPase}$ activity and oligomerization are not affected in mio10 $\Delta$ mutants. (A) Wildtype and mio10 $\Delta$ mitochondria were solubilized in $1 \%$ digitonin buffer and subjected to BN-PAGE (4-10\% gradient gel). In-gel activity assays were carried out as described in

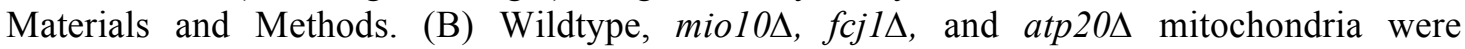
solubilized in $0.35 \%$ digitonin buffer and subjected to BN-PAGE (3-13\% gradient gel). In-gel $\mathrm{F}_{1} \mathrm{~F}_{\mathrm{O}} \mathrm{ATPase}$ activity assay was performed. 


\subsection{Identification of Mio10 and MINOS1 interaction partners}

Results so far indicate that Mio10 and MINOS1 are not involved in $\mathrm{F}_{1} \mathrm{~F}_{\mathrm{O}} \mathrm{ATPase}$ dimerization, oligomerization, or activity. A function of the proteins remains unclear. To this end, interaction partners of Mio10 and MINOS1 were isolated and analyzed by mass spectrometry with the objective of elucidating Mio10/MINOS1 functions.

\subsubsection{Identification of Mio10 interaction partners}

In order to identify interaction partners of Mio10, the MIO10 ORF was C-terminally tagged with a streptavidin tag followed by a FLAG tag (SF). Yeast cells were harvested and mitochondria were isolated. Using the Mio $10^{\mathrm{SF}}$, a large-scale protein isolation was performed via the streptavidin tag, and eluates were analyzed by mass spectrometry. First, eluates from the Mio $10^{\mathrm{SF}}$ isolation were subjected to SDS-PAGE and the gel was stained with coomassie. The SDS-PAGE was cut into 23 slices, which were then analyzed by high-resolution online liquid chromatography-tandem mass spectrometry (LC-MS/MS). Co-isolated proteins were scored based on the normalized fold change calculated using a label-free spectral count approach. Table 7 indicates specific interacting proteins with highest spectral counts, in which a normalized fold change cut-off of 0.1 was applied. The normalized fold change was calculated based on spectral counts of a specific protein and total spectral counts for all proteins in the sample. Besides the bait Mio10, Fcj1, which is involved in mitochondrial cristae formation and maintenance, had the highest sample spectral count (Rabl et al., 2009). Moreover, none of the highest scoring proteins were $\mathrm{F}_{1} \mathrm{~F}_{\mathrm{O}} \mathrm{ATP}$ ase associated proteins.

Indeed, the LC-MS/MS results provide a wide range of proteins, of both characterized and previously uncharacterized functions. Characterized proteins identified in the analysis range from cytochrome $c$ oxidase components (Cox20), to mitochondrial import (Pam17), mitochondrial translational regulation (Mdm38), and mitochondrial ribosomal proteins (Mrp127). These proteins, however, gave low spectral counts and their identification might be a result of nonspecific binding to the column. Interestingly, many of the highest scoring proteins (YGR235C, Aim37, Aim13, Aim46, Aim5, and Aim24), are previously uncharacterized and may be potential interacting partners of Mio10. The AIM (Altered Inheritance of Mitochondria) proteins were found by a computational screen to play a role in 
mitochondrial biogenesis and inheritance (Hess et al., 2009). Their interaction with Mio10 may indicate a possible role of Mio10 in mitochondrial inheritance.

Table 7. Identified proteins of the Mio10 ${ }^{\mathrm{SF}}$ Strep Tactin-chromotography. The raw MS files were searched and analyzed with Mascot (Mascot Deamon version 2.2.2, Matrix Science) and Scaffold 3 (Proteome Software) using the S. cerevisiae NCBI non-redundant protein database. The normalized fold change ratios were then calculated in Scaffold using the spectral count approach (Liu et al., 2004). Mass spectrometric analysis performed by Monika Raabe and Miroslav Nikolov (AG Urlaub, MPI-bpc, Göttingen).

\begin{tabular}{|c|c|c|c|c|c|c|}
\hline \multirow{2}{*}{ Protein } & \multirow{2}{*}{$\begin{array}{l}\text { Molecular } \\
\text { weight }\end{array}$} & \multirow{2}{*}{$\begin{array}{c}\text { Normalized } \\
\text { fold } \\
\text { change } \\
\text { (spectrum } \\
\text { count) }\end{array}$} & \multicolumn{2}{|c|}{ Spectral counts } & \multicolumn{2}{|c|}{ Unique peptides } \\
\hline & & & Control & Sample & Control & Sample \\
\hline Fcj1 & $61 \mathrm{kDa}$ & 0.09 & 26 & 614 & 16 & 59 \\
\hline Mio10 & $10 \mathrm{kDa}$ & 0.07 & 6 & 143 & 3 & 11 \\
\hline YGR235C & $27 \mathrm{kDa}$ & 0 & 0 & 136 & 0 & 26 \\
\hline Aim37 & $27 \mathrm{kDa}$ & 0.05 & 2 & 126 & 2 & 25 \\
\hline Nde2 & $62 \mathrm{kDa}$ & 0.02 & 0 & 75 & 0 & 26 \\
\hline Aim13 & $19 \mathrm{kDa}$ & 0.1 & 4 & 52 & 3 & 19 \\
\hline Aim46 & $34 \mathrm{kDa}$ & 0.1 & 3 & 43 & 3 & 20 \\
\hline Aim5 & $12 \mathrm{kDa}$ & 0.1 & 3 & 40 & 3 & 11 \\
\hline Odc1 & $34 \mathrm{kDa}$ & 0.05 & 1 & 31 & 1 & 19 \\
\hline Aim38 & $25 \mathrm{kDa}$ & 0 & 0 & 29 & 0 & 12 \\
\hline Cox20 & $24 \mathrm{kDa}$ & 0.07 & 1 & 22 & 1 & 12 \\
\hline Cbp4 & $18 \mathrm{kDa}$ & 0 & 0 & 20 & 0 & 11 \\
\hline YBL095W & $31 \mathrm{kDa}$ & 0 & 0 & 17 & 0 & 11 \\
\hline Aim31 & $18 \mathrm{kDa}$ & 0 & 0 & 16 & 0 & 11 \\
\hline Yhb1 & $45 \mathrm{kDa}$ & 0.1 & 1 & 13 & 1 & 8 \\
\hline Aim24 & $44 \mathrm{kDa}$ & 0.1 & 1 & 11 & 1 & 8 \\
\hline Mdm38 & $65 \mathrm{kDa}$ & 0.1 & 1 & 10 & 1 & 7 \\
\hline Oms1 & $56 \mathrm{kDa}$ & 0 & 0 & 9 & 0 & 7 \\
\hline Cir2 & $70 \mathrm{kDa}$ & 0 & 0 & 9 & 0 & 8 \\
\hline Pam17p & $22 \mathrm{kDa}$ & 0 & 0 & 9 & 0 & 4 \\
\hline Fmp33 & $20 \mathrm{kDa}$ & 0.1 & 1 & 9 & 1 & 4 \\
\hline Tuf1 & $48 \mathrm{kDa}$ & 0 & 0 & 8 & 0 & 6 \\
\hline Mcr1 & $34 \mathrm{kDa}$ & 0 & 0 & 5 & 0 & 4 \\
\hline Aim45 & $37 \mathrm{kDa}$ & 0 & 0 & 5 & 0 & 4 \\
\hline Pet100 & $13 \mathrm{kDa}$ & 0 & 0 & 5 & 0 & 3 \\
\hline Sod2 & $24 \mathrm{kDa}$ & 0 & 0 & 5 & 0 & 3 \\
\hline Mrpl27 & $16 \mathrm{kDa}$ & 0 & 0 & 5 & 0 & 3 \\
\hline
\end{tabular}


A

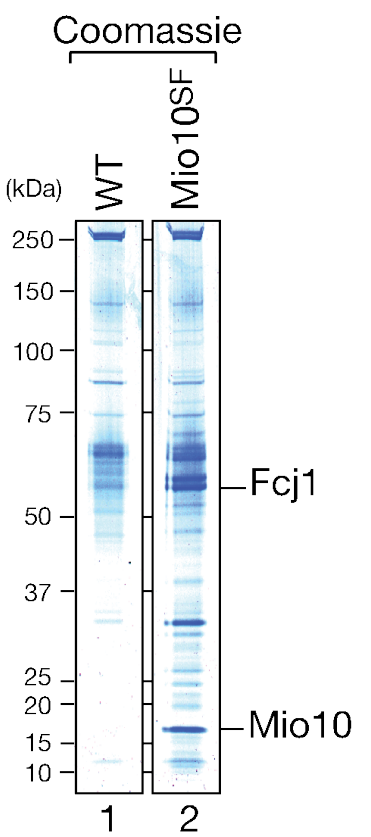

B

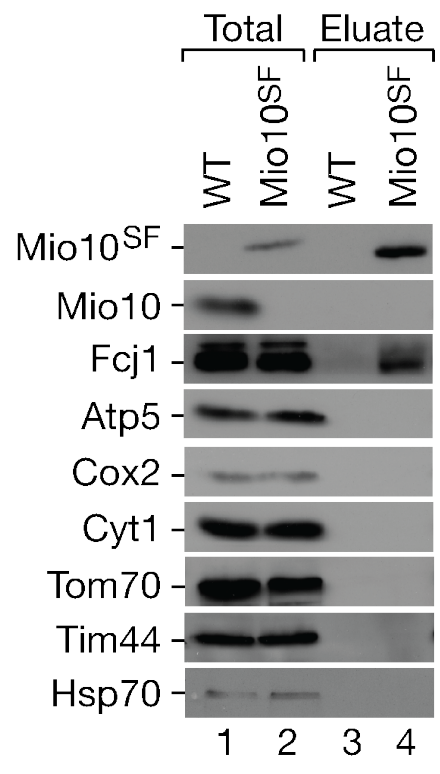

C

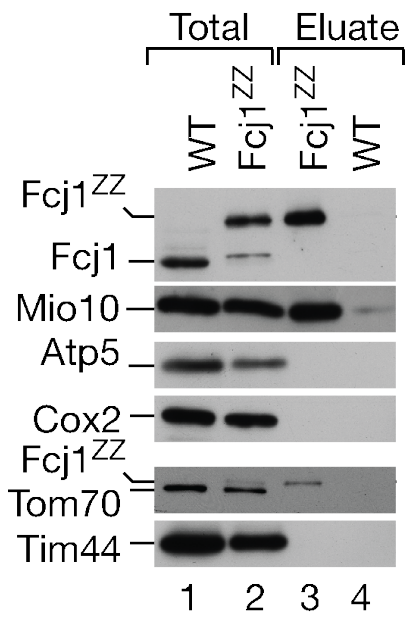

Figure 26. Mio10 interacts with Fcj1. (A) Wildtype and Mio10 ${ }^{\mathrm{SF}}$ mitochondria were solubilized and subjected to Strep-Tactin chromatography. Eluates were separated by SDSPAGE and gels were coomassie stained. (B) Eluates from Strep-Tactin chromatography were analyzed by SDS-PAGE and Western blotting. (C) Wildtype and Fcj1 $1^{\mathrm{zz}}$ mitochondria were solubilized and subjected to IgG chromatography. Eluates were analyzed by SDS-PAGE and Western blotting.

The protein composition of coomassie stained bands was identified due to the availability of spectral counts for each slice. Figure 26A depicts the coomassie stained gel used for LC-MS/MS analysis, in which coomassie stained bands corresponding to Fcj1 and Mio10 were annotated. The results from the Mio $10^{\mathrm{SF}}$ complex isolation and consequent analysis indicated that Fcj1 co-isolates with Mio10. To further verify this, eluates from the Mio $10^{\mathrm{SF}}$ isolation were separated on an SDS-PAGE and subjected to Western blot analysis (Figure 26B). Immunoblotting for Fcj1, indicate a clear band in Mio10 ${ }^{\mathrm{SF}}$ eluate and a lack of signal in the wildtype control, thus verifying results from the mass spectrometry. Moreover, immunodetection for Atp5 verified that the $\mathrm{F}_{1} \mathrm{~F}_{\mathrm{O}} \mathrm{ATPase}$ is not significantly isolated with Mio10, whereas negative controls such as respiratory chain proteins; Cyt1 (complex III) and Cox 2 (cytochrome $c$ oxidase) and mitochondrial import machinery (Tom70 and Tim44) were all absent. Moreover, Western blot analysis indicates that the Mio $10^{\mathrm{SF}}$ strain does not contain any endogenous non-tagged Mio10. To further confirm Fcj1 interaction with Mio10, 
mitochondrial proteins were immunoprecipitated from an Fcj1 ${ }^{\mathrm{zz}}$ strain (Figure 26C). Results confirm that Mio10 co-isolates with Fcj1, whereas controls such as Atp5, Cox2, Tom70, and Tim44 were negative. A Mio10 interaction with Fcj1 may indicate a role in cristae biogenesis (Rabl et al., 2009).

\subsubsection{Identification of MINOS1 interaction partners}

Isolation of Mio10-containing complexes followed by mass spectrometric analysis revealed that Mio10 interacts with Fcj1 rather than the $\mathrm{F}_{1} \mathrm{~F}_{\mathrm{O}}$ ATPase as originally hypothesized. To investigate whether the interaction is conserved across higher eukaryotes, a SILAC (stable issotope labeling of amino acids in cell culture) combined with mass spectrometry approach was carried out (Figure 27). SILAC makes use of differential isotope labeling to achieve quantitative based mass spectrometric analysis, and has been proven useful in various proteomic applications (Ong et al., 2002; Ong and Mann, 2006). In brief, HEK293T cells were grown in media containing either light or heavy labeled lysine and arginine. Cultured cells were then harvested, mitochondria isolated, and consequently solubilized in a mild digitonin buffer. MINOS1-containing mitochondrial protein complexes were then immunoprecipitated by a MINOS1 specific antibody from heavy isotope containing cells, whereas a control antibody was used to immunoprecipitate proteins from light isotope containing cells. Eluates from cells with light or heavy isotope containing medium were combined and subjected to SDS-PAGE analysis; at which point the coomassie stained gel was sliced and subject to LC-MS/MS. In addition, for statistical analysis and to rule out possible false positives/negatives, the reverse (label switch) experiment was performed. In this case, MINOS1-specific antibodies were used to immunoprecipitate light isotope containing cells, whereas unspecific antibodies were used for the heavy isotope containing cells.

Spectral counts normalized ratios of heavy-over-low (H/L) for the forward and reverse experiments are presented in (Figure 28A). Enriched proteins, which satisfy the set threshold of 2.0, are labeled with a red dot whereas nonspecific/non enriched (under threshold of 2.0) are labeled with blue dots. The dot-blot clearly indicates that Mitofilin (human homolog of Fcj1) has the highest score, supporting the notion that the Mio10-Fcj1 interaction in yeast is conserved in human. Interestingly, mass spectrometric analysis also indicates that many of the known Mitofilin interacting proteins: CHCHD3, HSPA9, and DNJAC1, SAM50, MTX1, and MTX2 (green dot 
with red circumference) are also immunoprecipitated with Mitofilin (Xie et al., 2007; Darshi et al., 2011). In addition, various other mitochondrial and non-mitochondrial proteins were enriched with MINOS1. Some of these interactions were verified by Western blot analysis of the immunoprecipitated protein samples (Figure 28B). MINOS1 specific antibodies ( $\alpha$-MINOS1) efficiently precipitated MINOS1, Mitofilin (IMMT), CHCHD3, and HSPA9 compared to the control antibodies. In parallel to yeast mass spectrometric analysis, MINOS1-specific antibodies do not precipitate the $\mathrm{F}_{1} \mathrm{~F}_{\mathrm{O}} \mathrm{ATPase} \mathrm{ATP} 2$ protein, further indicating that MINOS1 is not an $\mathrm{F}_{1} \mathrm{~F}_{\mathrm{O}} \mathrm{ATPase}$ associated protein. Other possible interactions could not be verified due to the unavailability of antibodies. Nonetheless, mass spectrometric analysis of MINOS1 interactions indicate that MINOS1 interacts with Mitofilin, indicating that the Mio10/Fcj1 interaction is conserved across higher eukaryotes.

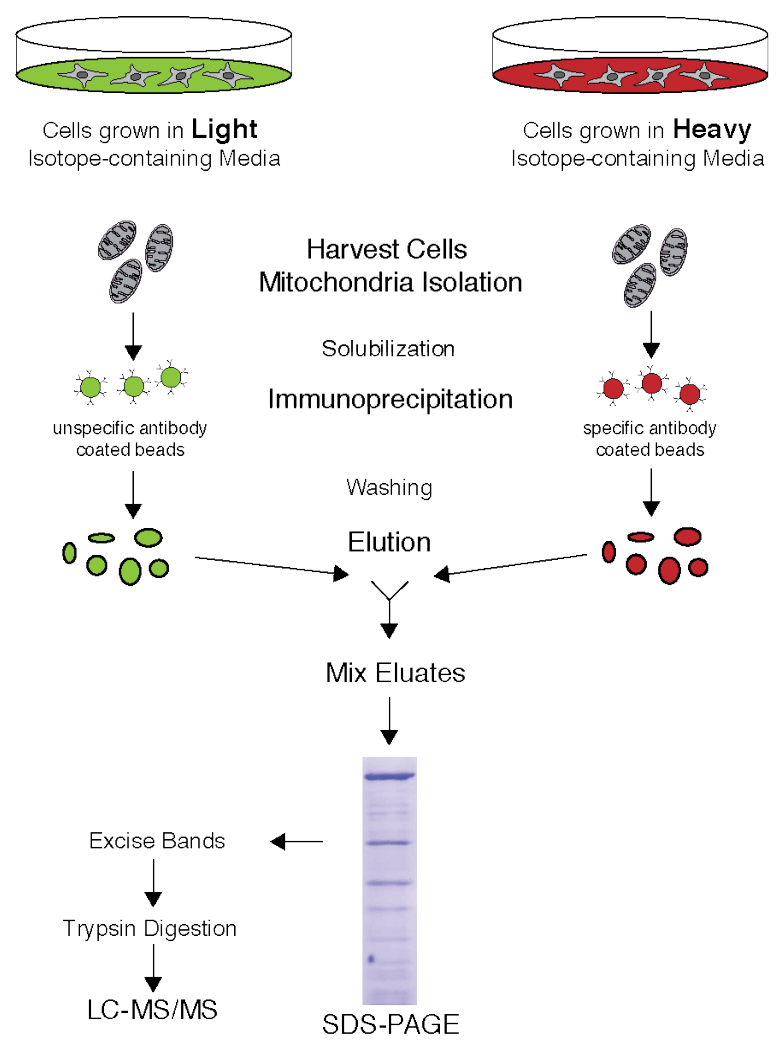

Figure 27. Schematic of the SILAC approach and mass spectrometric analysis. Figure courtesy of Markus Deckers. HEK29T cultured cells were grown in either Light or Heavy isotope containing medium. Mitochondria from each culture were isolated, solubilized and complexes were immunoprecipitated with either MINOS1 specific antibodies or control antibodies. After washing elutions were mixed and subjected to SDS-PAGE. The gel was cut into 23 equal and in-gel trypsinization was performed, followed by LC-MS/MS analysis. 
A

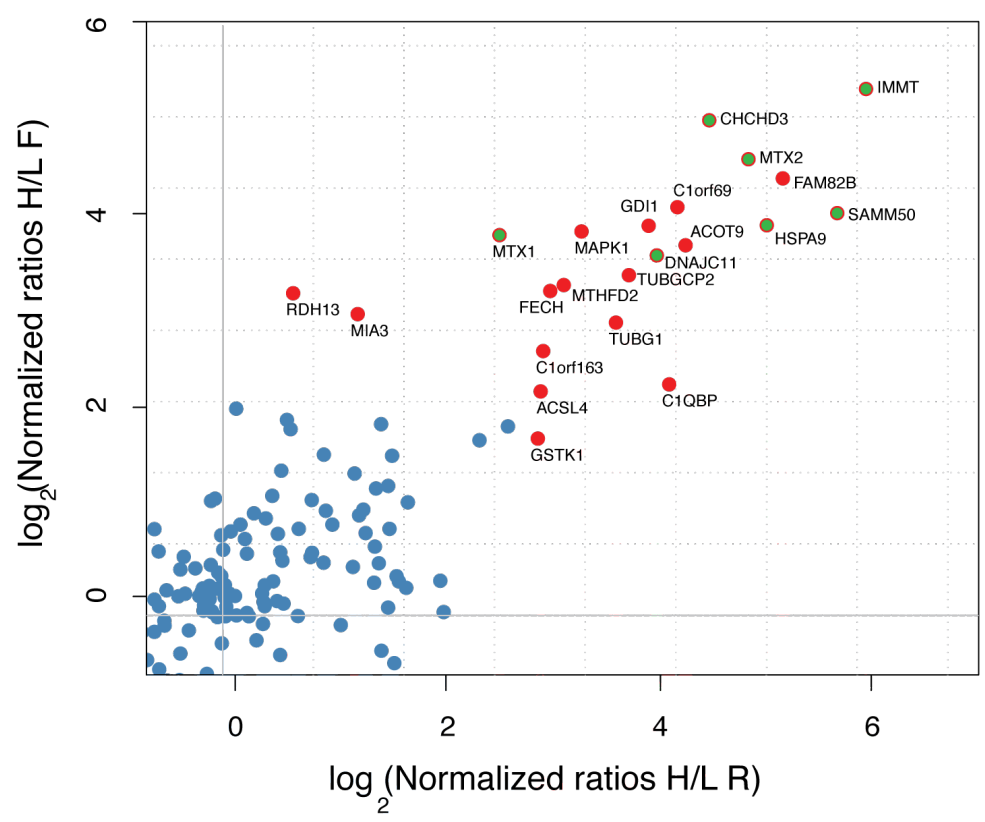

B

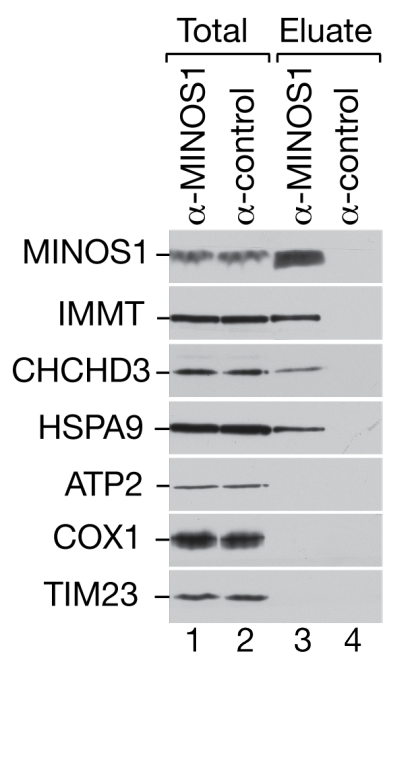

Figure 28. MINOS1 interacts with Mitofilin. Identification of MINOS1 associated proteins by co-immunoprecipitation and SILAC-MS. (A) RAW MS files from LC-MS/MS were analyzed by MaxQuant and Mascot using the IPI human protein database. Red dots indicate enriched proteins characterized by a high normalized ratio of heavy over light values $(\mathrm{H} / \mathrm{L})$. Forward $\mathrm{F}(\mathrm{H}=\mathrm{MINOS} 1 / \mathrm{L}=$ Control), Reverse $\mathrm{R}(\mathrm{H}=$ control/L=MINOS1, reverse ratios were inversed for plotting). Green dots with red circumference indicate known Mitofilin interacting proteins. (B) Isolated mitochondria from HEK293T cells were solubilized and subjected to Coimmunoprecipitation with MINOS1 and control antibodies. Eluates were separated by SDSPAGE and analyzed by Western blotting. IMMT, Mitofilin. Mass spectrometric analysis performed by Miroslav Nikolov (AG Urlaub, MPI-bpc, Göttingen).

\subsection{Characterization of Mio10 and MINOS1 interaction with Fcj1 and Mitofilin}

To assess Mio10 and MINOS1 with respect to Fcj1 and Mitofilin complexes, solubilized wildtype mitochondria were subjected to size exclusion chromatography. Proteins were eluted in 12 fractions and subjected to SDS-PAGE and Western blot analysis (Figure 29A). Mio10 appears to form various sized complexes: a smaller complex at approximately $232 \mathrm{kDa}$ (fraction 8) and a larger complex at approximately 1.2 $\mathrm{MDa}$ (fraction 3). The same holds true when examining Fcj1-containing complexes, which appear to elute at the same fractions as Mio10. This further supports the Fcj1 and Mio10 interaction. Moreover, it may be inferred from the size exclusion 
chromatography that together, both Mio10 and Fcj1 form a large complex. It appears that the complex is larger than respiratory chain complexes formed by cytochrome $c$ oxidase and $\mathrm{F}_{1} \mathrm{~F}_{\mathrm{O}} \mathrm{ATPase}$ as indicated by immunoblotting for Cox 1 and Atp5 respectively. Furthermore, $\mathrm{F}_{1} \mathrm{~F}_{\mathrm{O}} \mathrm{ATPase}$ signals (Fractions 4-7) are devoid of Mio10 indicating that Mio10 does not associate with the $\mathrm{F}_{1} \mathrm{~F}_{\mathrm{O}} \mathrm{ATPase}$.

A

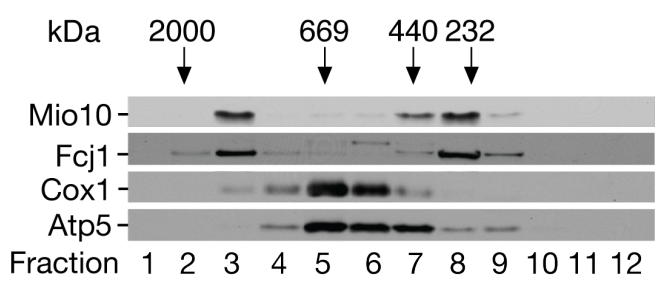

B

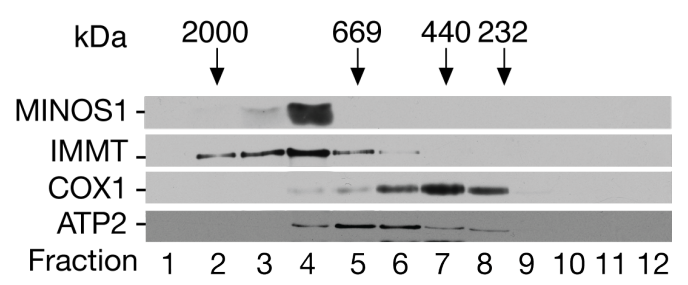

Figure 29. Mio10/Fcj1 and MINOS1/Mitofilin form large mitochondrial complexes. Wildtype yeast (A) and HEK29T (B) mitochondria were solubilized and subjected to size exclusion chromatography using a ÄKTApurifier system (GE Healthcare). The resulting fractions from the ÄKTApurifier system were TC precipitated, dried and subjected to SDSPAGE and Western blotting. IMMT= Mitofilin.

MINOS1 containing complexes were analyzed as well by size exclusion chromatography (Figure 29B). In parallel to Mio10 in yeast, MINOS1 forms as well a large predominant protein complex of approximately $1 \mathrm{MDa}$ (fractions 3-4), whereas a smaller complex is absent. Mitofilin also appears to form a large complex in the MDa range (fraction 2-5), predominantly in fraction 4 and thus appears in complex with MINOS1. The size of the MINOS1/Mitofilin complex appears to be distinctive from cytochrome $c$ oxidase containing complexes (fraction 7) and $\mathrm{F}_{1} \mathrm{~F}_{\mathrm{O}} \mathrm{ATPase}$ complexes (fraction 5). The results from size exclusion chromatography thus indicate that Mio10 and MINOS1 are part of a large mitochondrial complex that contains Fcj1 in yeast and Mitofilin in higher eukaryotes. 


\subsection{Investigation of the role of Mio10 in mitochondrial morphology}

As mass spectrometric analysis of yeast and human mitochondria in combination with size exclusion chromatography indicate that Mio10 and MINOS1 interact with Fcj1 and Mitofilin in large mitochondrial complexes. Previous reports indicate that Fcj1 and Mitofilin are involved in cristae biogenesis and inner membrane morphology (John et al., 2005; Rabl et al., 2009). The question, whether Mio10 or MINOS1 are also involved in mitochondrial inner membrane organization, was asked. Due to the availability of established tool and deletion strains, a role of the proteins were investigated primarily in yeast. $M I O 10$ deletion strains were again used to investigate whether Mio10, like Fcj1 is also involved in mitochondrial morphology. MIO10 and FCJ1 deletions strains were further analyzed by live-cell fluorescence microscopy and electron microscopy. In all cases, an ATP20 deletion strain was also analyzed for comparison.

\subsubsection{Growth test: SG /SD medium at $18^{\circ} \mathrm{C}$}

MIOIO and FCJ1 deletion mutant cells were grown on minimal glucose (SD) and minimal glycerol (SG) medium plates at non-permissive temperatures of $18^{\circ} \mathrm{C}, 37^{\circ} \mathrm{C}$, as well as $30^{\circ} \mathrm{C}$ (Figure 30 ). Mutant $f_{c j} 1 \Delta$ cells have been shown to exhibit a growth defect when grown on YLac in which deletion of FCJ1 leads to altered mitochondrial inner membrane morphology, mainly by affecting numbers of cristae tips (Rabl et al., 2009). Growth behavior analysis with SD and SG were used to enforce stringent growth conditions. Indeed, mutations affecting inner membrane stability are more potent at low or high non-permissive temperatures (Brandner et al., 2005; Rabl et al., 2009). Whereas wildtype and mutant cells all grow similarly on SD, differences are exhibited mainly when grown on SG medium. At $30^{\circ} \mathrm{C}$, mio $10 \Delta$, atp $20 \Delta$, and $f c j 1 \Delta$ exhibit an impeded growth compared to wildtype cells on SG medium. Moreover, stark differences can be observed at $18^{\circ} \mathrm{C} \mathrm{SG}$, at which miolos and $f c j 1 \Delta$ exhibit a

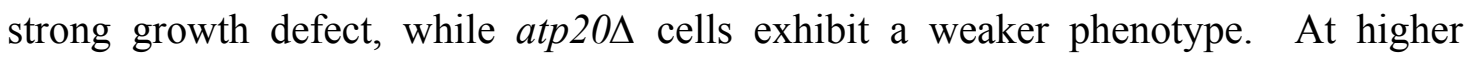
temperatures, $37^{\circ} \mathrm{C}$, mutant cells again exhibit a growth phenotype, although not as prominent as in $18^{\circ} \mathrm{C}$. The similarity in miolos and $f_{c j} 1 \Delta$ highlights a possible genetic and/or functional relationship. As growth on SG, is not completely inhibited at $30^{\circ} \mathrm{C}$ or $37^{\circ} \mathrm{C}$, a role in mitochondrial respiration is considered unlikely. 


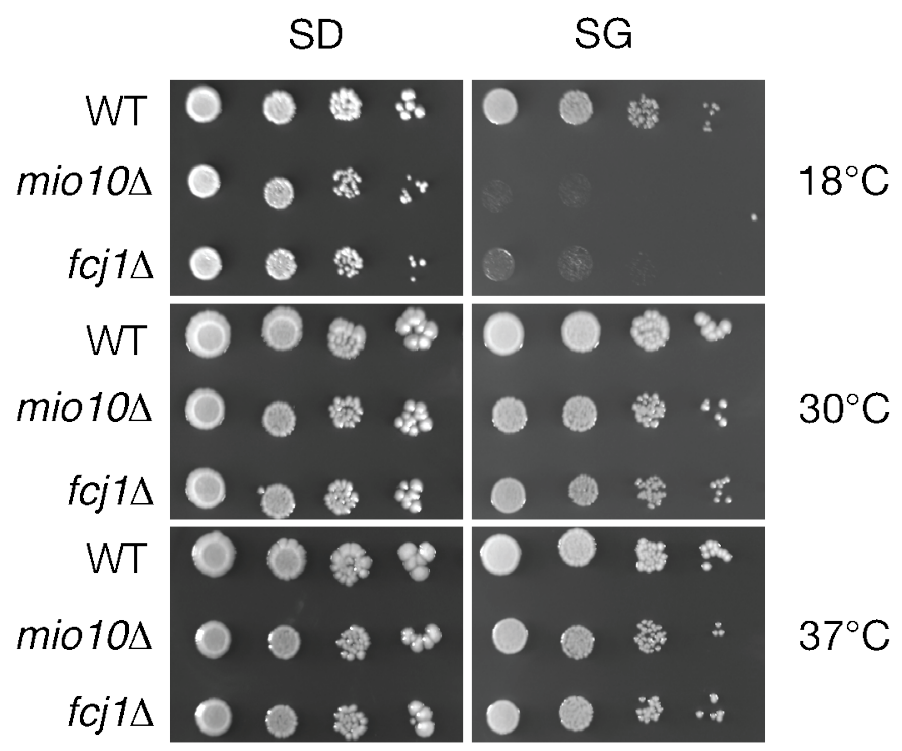

Figure 30. MIO10 and FCJ1 deletion mutants' growth defect at low temperatures. Wildtype, mio $10 \Delta$, and $f_{c j} 1 \Delta$ yeast cells were spotted in serial 10 -fold dilutions on minimal fermentable (SD) and non-fermentable (SD) minimal media. Cells were grown at $18^{\circ} \mathrm{C}, 30^{\circ} \mathrm{C}$, and $37^{\circ} \mathrm{C}$.

\subsubsection{Protein steady state analysis of mitochondria grown at $18^{\circ} \mathrm{C}$}

Mitochondria were harvested from $M I O 10$ and FCJ1 deletion mutant cells grown at $18^{\circ} \mathrm{C}$ in SG medium. The steady-state protein levels were analyzed by SDS-PAGE (Figure 31A) and BN-PAGE (Figure 31B). The deletion strains are confirmed by the absence of Mio10 and Fcj1 signals in respective strains. One should note a signal detection migrating above Fcj1 signal in $f c j 1 \Delta$ cells; this corresponds to an antibody cross reaction rather than residual Fcj1 protein. Moreover, Fcj1 protein levels appear to be slightly reduced in miol0s compared to wildtype. This effect, however, is not paralleled in $f_{c j} 1 \Delta$ mutant cells, which have levels of Mio10 comparable to wildtype cells. Furthermore, steady-state levels of respiratory chain proteins (Rip1, Cty1, Cox2, Atp5) are not affected, whereas outer membrane proteins (Tom70, Porin) and the inner membrane protein Tim21 are also unaffected by the absence of Mio10 or Fcj1. Assembly of respiratory chain complexes in miol0s grown at $18^{\circ} \mathrm{C}$ in $\mathrm{SG}$ medium was accessed by BN-PAGE, at which assembly of the $\mathrm{F}_{1} \mathrm{~F}_{\mathrm{O}} \mathrm{ATPase}$ and complex III appear unaffected. 


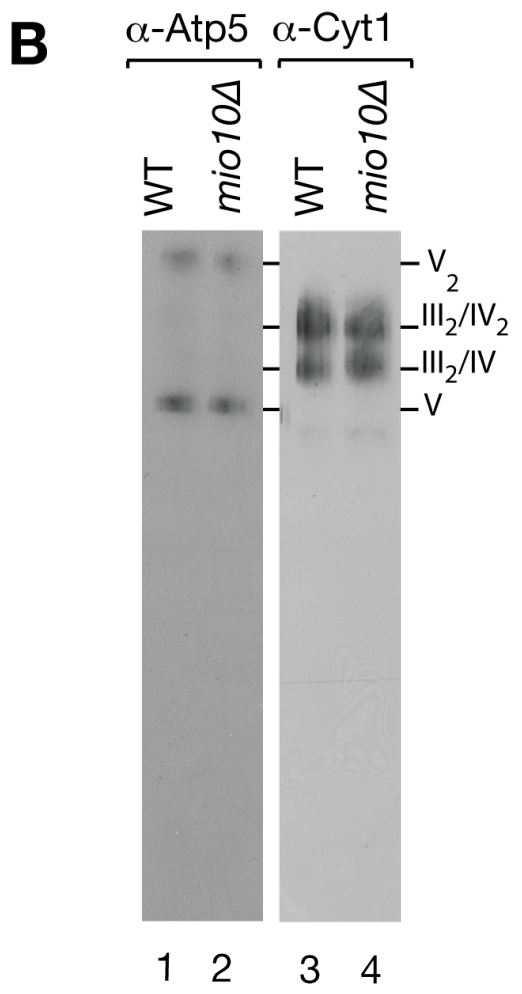

Figure 31. Protein steady-state levels are unaffected in mio10 $\Delta$ mutants. (A) Wildtype, mio10 $\Delta$, and fcils mitochondria were subjected to SDS-PAGE and Western blotting. (B)

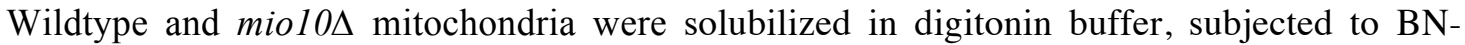
PAGE (4-10\% gradient gel) and Western blotting.

\subsubsection{Live-cell fluorescence microscopy analysis of mio10 $\Delta$ mitochondria}

Mitochondria form interconnected dynamic networks of which phenotypic characteristics are dependent on the metabolic state of the cell and a balance between the processes of mitochondrial fission and fusion (Okamoto and Shaw, 2005). The organization of the mitochondrial network of mio10 $\Delta$ mutant yeast cells was visualized by live-cell imaging (Figure 32), in which yeast cells were transformed with a mitochondrial localized GFP expressing plasmid, pVTU100U-mitoGFP (Westermann and Neupert, 2000). This allows contrasting bright fluorescent mitochondria against clear background staining, and thus proper assessment of network morphology. Wildtype mitochondria display a typical reticular mitochondrial network which transverses the majority of the cell. On the other hand, atp20د mutant cells exhibit atypical networks characterized by thickened and fragmented mitochondria. Upon analysis of both mio10 $\Delta$ and $f c j 1 \Delta$ mutant cells, it is evident that they share a similar mitochondrial network organization. In both cases, mitochondrial networks appeared on the periphery of cell and more condensed than in the atp20د counterpart strain. 
Indeed, analysis of $f c j 1 \Delta$ mitochondria is in agreement with previously published observations (Rabl et al., 2009). The similarities observed in the mitochondrial networks for miol0s and $f c j 1 \Delta$ strains may support the notion of a functional relationship between the two proteins.

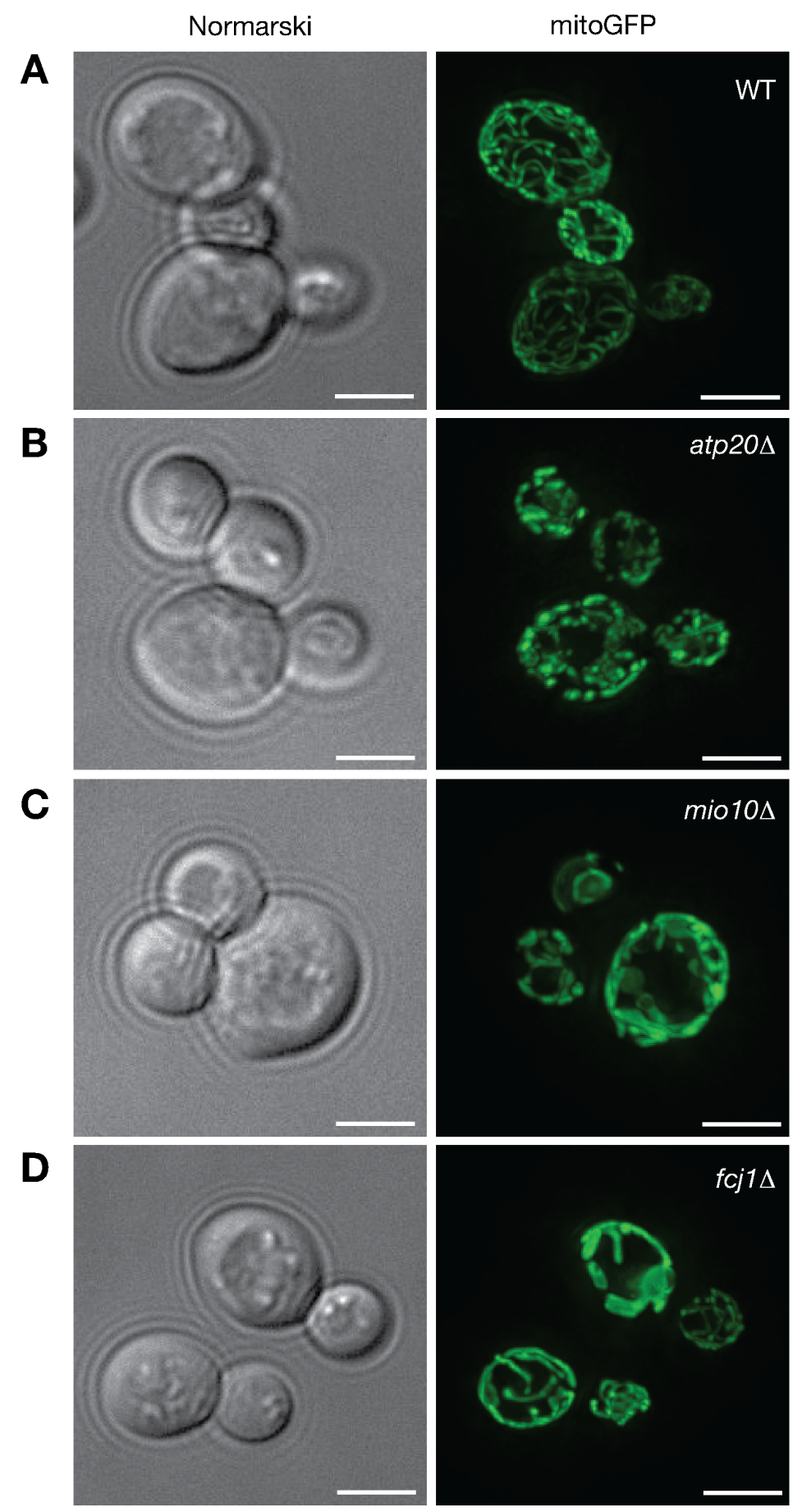

Figure 32. Mitochondrial morphology is altered in mio10s. Yeast wildtype, atp204, mio10 1 and $f c j 1 \Delta$ cells transformed with plasmid pVT100U-mitoGFP and analyzed by fluorescence microscopy. Scale Bars, $2.5 \mu \mathrm{m}$. Microscopy was performed by Daniel Jans (AG Jakobs, MPI-bpc, Göttingen). 


\subsubsection{Analysis of mio10 $\Delta$ mitochondria by electron microscopy}

The mitochondrial cristae morphology of wildtype and mutant yeast cells were further assessed with the use of electron microscopy coupled with high-pressure freezing, HPF, in order to preserve intact cellular structure (Figure 33A). Wildtype cells contain mitochondria with typical tubular cristae membranes whereas atp20 mitochondria appear to have the distinctive 'onion-like' inner membrane organization, previously shown for atp20 and atp21s mutants (Paumard et al., 2002; Arselin et al., 2004). Mitochondria of the ATP20 deletion mutant contain two or three lamellar cristae membranes, which span the entire organelle in a spherical manner. In contrast, analysis of miol0s and $f_{c j} 1 \Delta$ mitochondria indicated atypical yet less severe phenotype characterized by more compact transversion of the inner membrane. Moreover, in mio $10 \Delta$ and $f c j 1 \Delta$ cells, mitochondria appear to localize to the periphery of the cells, reflecting observations by live-cell fluorescent imaging.

The distinctive mitochondrial phenotypes observed from electron microscopy images where further subjected to a quantitative analysis to better define the various mutants (Figure 33B). An average of 55 images from each strain was used in the analysis, which is based on the percentage of analyzed cells that contain the various phenotypes. The tubular wildtype phenotype was termed 'normal', where as onionlike phenotype as simply 'onion-like'. The atypical phenotypes observed in miol0s and $f c j 1 \Delta$ were termed 'intermediate'. Quantification of the various mutant phenotypes indicates that a large majority (approximately 90\%) of wildtype mitochondria favor 'normal' inner membrane arrangements with $10 \%$ having either intermediate or onion-like structures. Interestingly, unlike the wildtype cells, none of the mutants appeared to have a similar inclination to one single phenotype and appeared to contain all three phenotypes in varying amounts. For instance, whereas

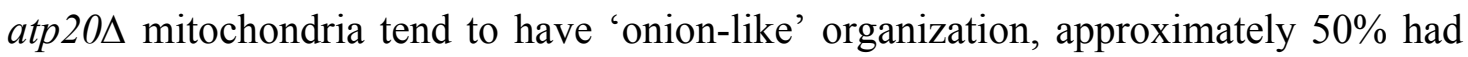
either normal or intermediate. The same holds true for $f c j 1 \Delta$ mutants, in which approximately $50 \%$ of the cells tend to have an 'intermediate' phenotype, with the other $50 \%$ either 'normal' or 'onion-like'. In contrast, miol0s mutants tend to favor 'intermediate' and 'onion-like' inner membrane organizations in rather similar quantities meanwhile approximately $20 \%$ were 'normal' in both strains. 
A
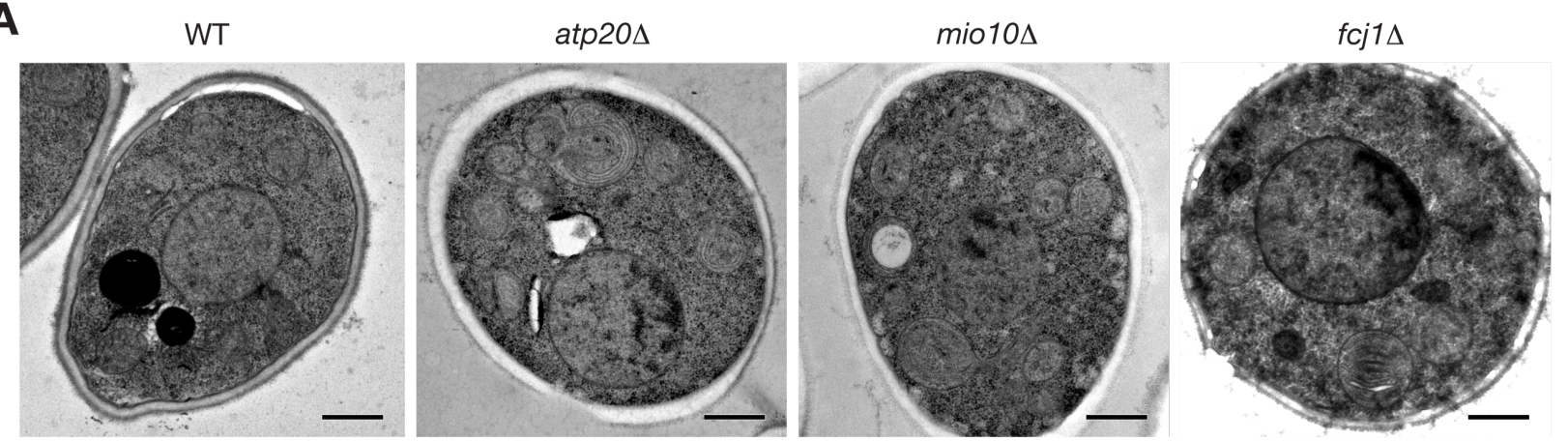

B
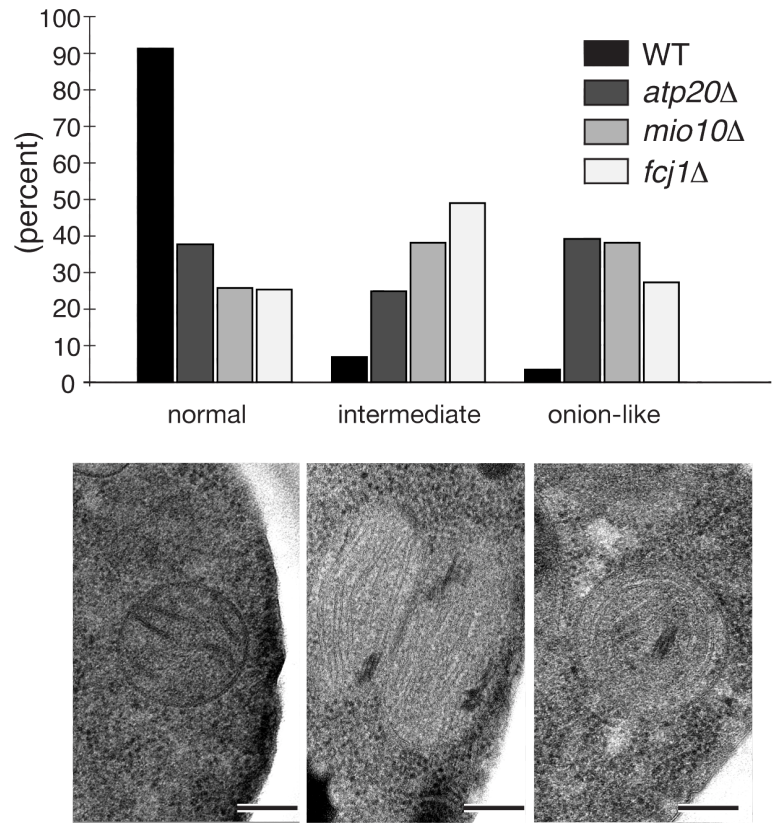

Figure 33. Aberrant cristae morphology in mio10 $\Delta$ mitochondria. (A) Electron microscopy of yeast wildtype, atp204, mio10 $\Delta$ and $f c j 1 \Delta$ cells after high pressure freezing (HPF). Bars, 1 $\mu \mathrm{m}$. (B) Quantitative analysis of different types of mitochondria phenotypes based on electron microscopy images from HPF fixed cells; wildtype WT $(\mathrm{n}=49)$, atp204 $(\mathrm{n}=51)$, mio104 $(\mathrm{n}=61)$ and $f c j 1 \Delta(\mathrm{n}=57)$ cells. Detailed view representation of normal, intermediate and onion-like mitochondria types. Microscopy and analysis were performed in collaboration with Dietmar Riedel (Mpi-bpc, Göttingen) 
In order to have an improved visualization of mutant mitochondria inner membranes, cells were chemically fixed with $\mathrm{KMnO}_{4}$ before analysis by electron microscopy (Figure 34). Fixation by $\mathrm{KMnO}_{4}$ allows the 'unmasking' of proteinphospholipids complexes generating a higher membrane contract in electron micrographs (Bradbury 1960). Under these conditions, mitochondria from the atp204 strain again have the onion-like organization characterized by clear various concentric layers of inner membrane. Moreover, atp204 mitochondria appear to have poorly defined cristae, compared to the defined tubular cristae membrane in the wildtype strain. Conversely, mio10 $\Delta$ and $f c j 1 \Delta$ mitochondria contain closely stacked lamellar membrane sheets which transverse horizontally the organelle (rather than spherical transversions of the atp $20 \Delta$ mutants). Furthermore, miolos and $f_{c j} 1 \Delta$ contain no cristae and appear to lack any recognizable cristae tips.

The results from the electron microscopy analysis of $\mathrm{HPF}$ and $\mathrm{KMnO}_{4}$ fixed cells indicate that Mio10 plays a functional role in mitochondrial inner membrane organization. The ORF YCL057c-A is thus referred to Mio10 for mitochondrial inner membrane organizer, whereas its human homolog is referred to MINOS1, for mitochondrial inner membrane organizing system 1. The combination of topological analysis, mass spectrometric analysis and size exclusion chromatography, indicate that Mio10 and MINOS1 are novel interacting partners of Fcj1 and Mitofilin, and form a large inner membrane complex. This complex is termed MINOS, for Mitochondrial Inner membrane Organizing System. Mio10 and MINOS1 do not associate with the $\mathrm{F}_{1} \mathrm{~F}_{\mathrm{O}} \mathrm{ATPase}$ or play a role in its dimerization. However by forming the MINOS complex, Mio10 and MINOS1 appears to play an essential role in cristae morphology and inner membrane architecture. 
A
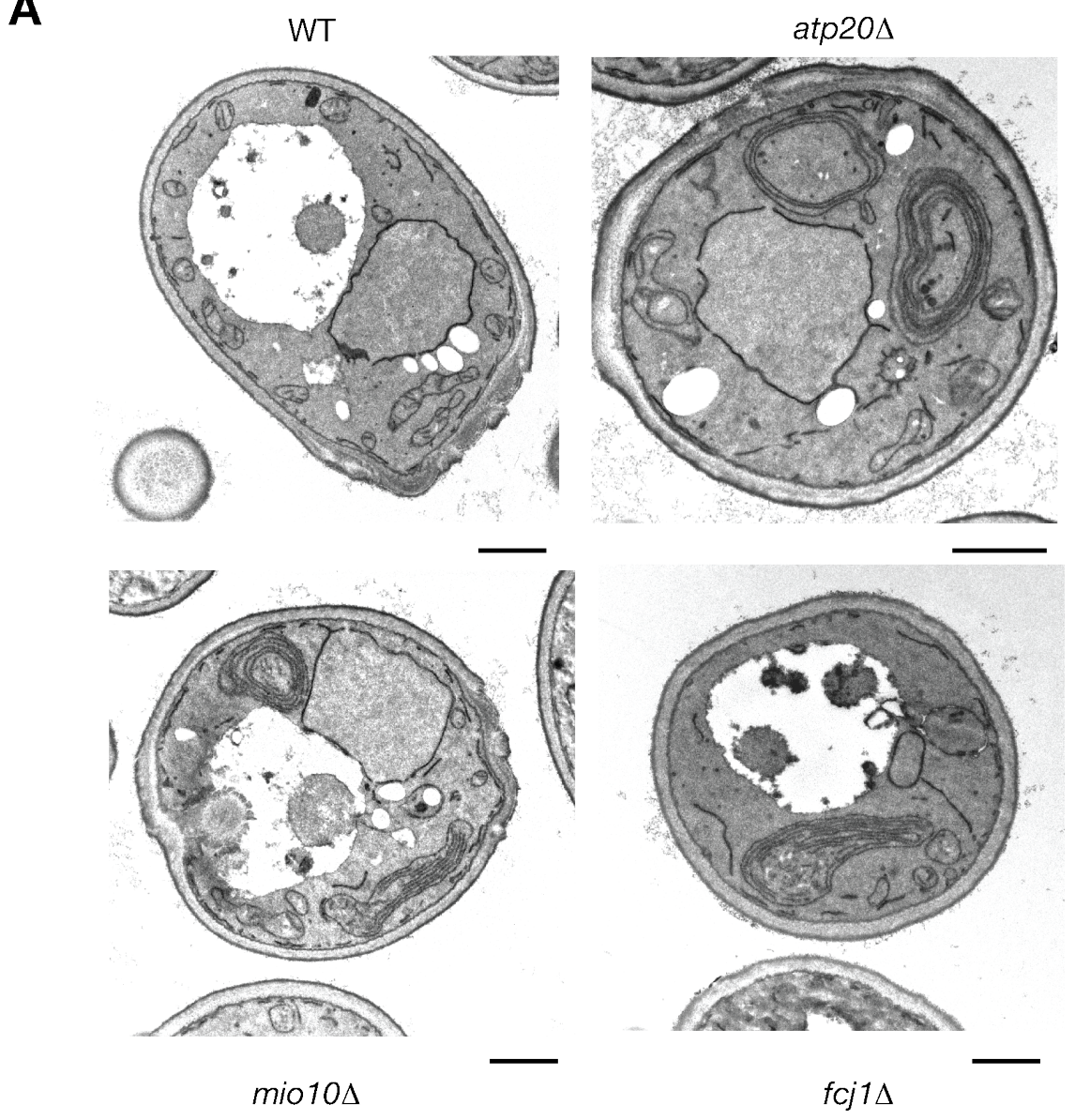

B
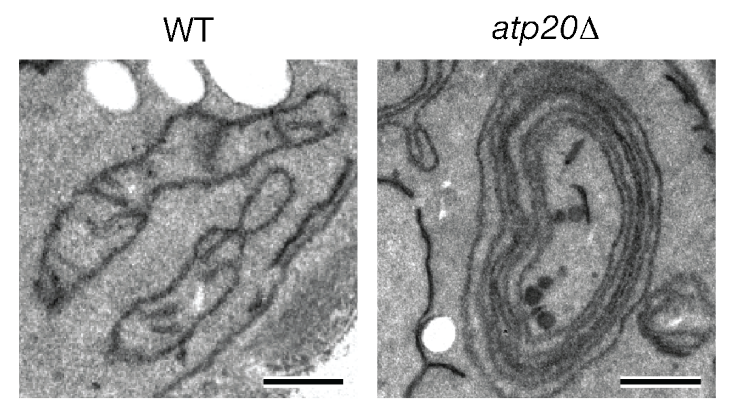

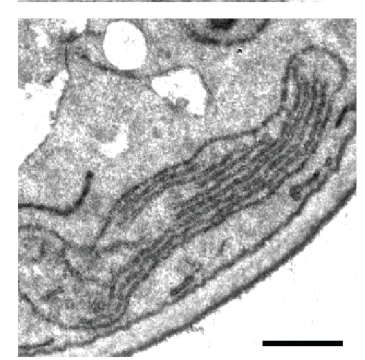

$\operatorname{mio10\Delta }$

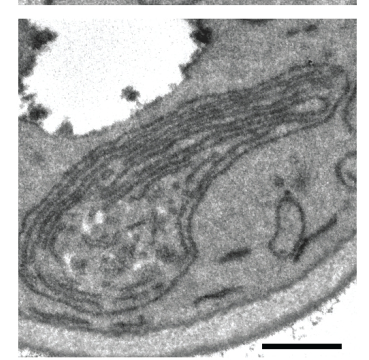

fcj $1 \Delta$

Figure 34. Absence of apparent cristae and cristae tips in mio10 $\Delta$ mitochondria. (A) Electron microscopy analysis of wildtype, atp204, mio104 and $f c j 1 \Delta$ cells after $\mathrm{KMnO}_{4}$ fixation. Scale bars, $200 \mathrm{~nm}$. (B) Detailed view of WT, atp204, mio10 and $f c j 1 \Delta$ mitochondria showed in (A). Microscopy and analysis were performed in collaboration with Wolfgang Schliebs (Ruhr Universität Bochum) 


\section{DISCUSSION}

In this study, an initial in silico approach to identify novel $\mathrm{F}_{1} \mathrm{~F}_{\mathrm{O}} \mathrm{ATPase}$ dimerization factors resulted in the identification of the yeast mitochondrial protein Mio10 as a potential candidate. Mio10, along with its human homolog, MINOS1, were investigated. These proteins were found to display a function unrelated to dimerization of $\mathrm{F}_{1} \mathrm{~F}_{\mathrm{O}} \mathrm{ATPase}$, and are instead novel factors involved in the maintenance of mitochondrial cristae morphology. Whereas recent studies have in parallel identified Mio10 in yeast, this is the first study that has identified MINOS1 as a novel interaction partner of Mitofilin in higher eukaryotes. The presented findings highlight the functional and evolutionary significance of the MINOS complex as a player in mitochondrial inner membrane architecture and as multifunctional component in eukaryotic mitochondria and potentially in other cellular organelles.

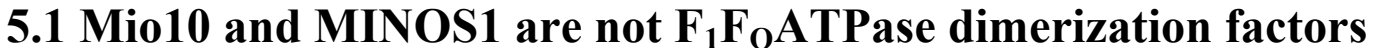

\subsubsection{Identification of the inner membrane proteins: Mio10 and MINOS1}

$\mathrm{F}_{1} \mathrm{~F}_{\mathrm{O}} \mathrm{ATPase}$ synthase dimers have been identified in various organisms ranging from yeast, mammals, and plants (Straus et al., 2008; Davies et al., 2011). Dimerization has various structural and functional roles. It contributes to the $\mathrm{F}_{1} \mathrm{~F}_{\mathrm{O}} \mathrm{ATP}$ ase holo-enzyme structure and plays a regulatory role in conditions of low electron transfer activity and reduced membrane potential (Buzhynskyy et al., 2007, Wittig and Schägger, 2009). Moreover, $\mathrm{F}_{1} \mathrm{~F}_{\mathrm{O}} \mathrm{ATPase}$ dimerization is also involved in the maintenance and biogenesis of cristae membranes (Giraurd et al., 2002; Paumard et al., 2002b; Gavin et al., 2004; Dudkina et al., 2006; Rak et al., 2007; Velours et al., 2009). Several $\mathrm{F}_{1} \mathrm{~F}_{\mathrm{O}} \mathrm{ATPase}$ subunits, such as subunit $\mathrm{a}, b, h$, and $y$, have been shown to contribute to dimerization in yeast and higher eukaryotes (Soubannier et al., 2002; Fronzes et al., 2003). In addition, several small $F_{O}$ interacting membrane proteins (Atp18, Atp19, Atp20, and Atp21) mediate dimeric interactions by binding sequentially at monomer interfaces in yeast (Arnold et al., 1997, 1998; Soubannier et al., 2002; Paumard et al., 2002a; Wagner et al., 2009, 2010). In contrast, these small dimerization factors have not been found in higher eukaryotes. Homologs of Atp20 and Atp21 are found in H.sapiens, however a functional role in $\mathrm{F}_{1} \mathrm{~F}_{\mathrm{O}} \mathrm{ATPase}$ dimerization has not been 
identified. The exact nature of what promotes $\mathrm{F}_{1} \mathrm{~F}_{\mathrm{O}} \mathrm{ATPase}$ monomer-monomer interactions and therefore dimerization is elusive and yet to be determined. To this end, the initial aim of this study was to identify novel $\mathrm{F}_{1} \mathrm{~F}_{\mathrm{O}} \mathrm{ATPase}$ dimerization factors in higher eukaryotic organisms that parallel the action of small dimerization factors in yeast.

In order to identify new dimerization factors, an in silico based analysis was performed based on characteristics of the primary amino acid sequences of identified factors. This included a protein with a small molecular size (less than $12 \mathrm{kDa}$ ) and at least one transmembrane domain. Subsequently, selecting proteins with GxxxG motifs and significant potential homologs narrowed search results down. The GxxxG motifs that are found in both Atp20 and Atp21, allow helix-helix membrane interactions with neighboring proteins (Russ and Engelman, 2000). Moreover, the GxxxG motif of Atp20 and Atp21 is critical for their role in dimerization (Arselin et al., 2003). The results of the in silico analysis yielded the ORF, YCL057c-A (Mio10). Blast search of the Mio10 primary amino acid sequence identified a potential human homolog, C1orf151 (MINOS1). The in silico analysis indicated a second potential dimerization factor, however Mio10/MINOS1 were selected based on the evidence that Mio10 (YCL057c-A) has been previously identified in large-scale proteomic screens and predicted to have a mitochondrial localization and that MINOS1 (C1orf151) has been found in a computational screen for regulators of oxidative phosphorylation (Sickmann et al., 2003; Reinders et al., 2006; Baughman et al., 2009). To this end, Mio10 and MINOS1 were then characterized biochemically and functionally.

The $\mathrm{F}_{1} \mathrm{~F}_{\mathrm{O}} \mathrm{ATPase}$ dimerization factors are membrane proteins that interact with the inner membrane embedded $F_{O}$ domain of $F_{1} F_{O} A T P a s e$ (Wagner et al., 2010). In order to investigate a possible role dimerization, the association of Mio10 and MINOS1 with the inner membrane was thus investigated. Subfractionation analysis was performed in tandem with membrane association assays using isolated yeast mitochondria. The results indicated that Mio10 is indeed an integral protein of the inner membrane. It was also inferred that Mio10 localizes to the inner membrane in a manner that its $\mathrm{N}$ and $\mathrm{C}$ termini are exposed to the IMS. One should note that this topology is based on the hypothetical model that Mio10 contains two transmembrane domains. Additional analysis is thus required to verify $\mathrm{N}$ terminal exposure to the IMS. 
This may include raising antibodies that specifically recognize the $\mathrm{N}$ terminus of Mio10. Furthermore, subfractionation analysis and membrane association assays of the human MINOS1 have also been performed using isolated human mitochondria from cultured HEK293T cells. The MINOS1 mitochondrial localization was also shown by confocal microscopy. These results indicated that MINOS1 is also a mitochondrial inner membrane protein that has membrane topology similar to yeast homolog.

\subsubsection{Mio10 and MINOS1 complexes comigrate but not physically associate with the $F_{1} F_{0} A T P a s e$}

To assess Mio10 and MINOS1 containing complexes, solubilized mitochondria were subjected to BN-PAGE after import and assembly. This approach has proven previously useful in the characterization of $\mathrm{F}_{1} \mathrm{~F}_{\mathrm{O}} \mathrm{ATPase}$ dimerization factors, as $\mathrm{BN}$ PAGE can be utilized to investigate large membrane-bound protein complexes under native conditions (Schägger and von Jagow, 1991; Nijtmans et al., 2002, Wagner et al., 2009, 2010). Radiolabeled Mio10 and MINOS1 precursors were synthesized in vitro and imported in yeast and human mitochondria. Mitochondria isolated from HEK293T human cultured cells were used as a representative model of higher eukaryotes. Upon analysis by BN-PAGE, both Mio10 and MINOS1 precursors were imported into either yeast or human mitochondria as well as assemble into complexes of various sizes, ranging from small (approximately $160 \mathrm{kDa}$ ) to large complexes (larger than $669 \mathrm{kDa}$ ). The observation that both protein precursors can be interchangeably imported into both yeast and human mitochondrial systems may reflect a high level of functional conservation. Indeed, sequence analyses of Mio10 and MINOS1 across various species indicated a high level of conservation mainly clustered at predicted transmembrane domains and particularly at the GxxxG motifs. Interestingly, both the $\mathrm{N}$ and $\mathrm{C}$ termini are not conserved. This may reflect that function and perhaps mitochondrial targeting and/or import are most likely dependent on the proteins' central domains. The notion whether Mio10 and MINOS1 can functionally complement each other is yet to be determined. Preliminary complementation assays, in which the MINOS1 coding gene was expressed under various promoters in a MIO10 deletion mutant, did not overcome growth defects observed (data not shown). Although MINOS1 does express in yeast as found by Western blot analysis, the failure to complement may be due to insufficient levels of 
MINOS1 overexpression and thus further optimizations are necessary. This may also indicate that whereas sequence differences may allow import of Mio10 and MINOS1 into in different species, these differences may not allow functional complementation.

Upon further analysis by BN-PAGE in which Mio10 radiolabeled precursor was imported in parallel with respiratory chain proteins, it appeared that the large Mio10 containing complexes comigrated with the $\mathrm{F}_{1} \mathrm{~F}_{\mathrm{O}} \mathrm{ATPase}$ dimer. In contrast, Mio10 containing complexes appeared larger than complex III or cytochrome $c$ oxidase containing complexes. Moreover, Mio10 import and assembly appeared to be dependent on the presence of the $\mathrm{F}_{1} \mathrm{~F}_{\mathrm{O}} \mathrm{ATPase}$ dimerization factors Atp20. In this case, the large Mio10 containing complexes failed to assemble in atp20د mitochondria. The effect was also shown to be specific to atp200 mitochondria and not due to nonspecific post lysis association. It should be noted that due to limitations of $\alpha$ MINOS1 and $\alpha$-Mio10 antibodies to recognize native epitopes on BN-PAGE, it was difficult to verify import and assembly results by Western blot analysis. As an alternative, second dimension analysis by SDS-PAGE was used. Surprisingly, second dimension analysis of Mio10 containing complexes in atp20د mitochondria revealed that the large Mio10 complex continues to be present in the absence of the $\mathrm{F}_{1} \mathrm{~F}_{\mathrm{O}} \mathrm{ATPase}$ dimer. This indicates that the Mio10 containing complex is different from the $\mathrm{F}_{1} \mathrm{~F}_{\mathrm{O}} \mathrm{ATPase}$ dimer. The Mio10 complex may thus have a similar size to the $\mathrm{F}_{1} \mathrm{~F}_{\mathrm{O}} \mathrm{ATPase}$ and may thus have similar migration properties on BN-PAGE. The Mio10 assembly defect in atp20 may possibly be an artifact. The severe effects that ATP20 deletion mutants have on the organization of the mitochondrial inner membrane may be a possible explanation for this. However, it is unclear why this specifically affects Mio10 assembly and not other $\mathrm{F}_{1} \mathrm{~F}_{\mathrm{O}} \mathrm{ATPase}$ components, such as Atp21, which assemble to the $\mathrm{F}_{1} \mathrm{~F}_{\mathrm{O}} \mathrm{ATPase}$ dimer as expected. In summary, analysis of Mio10 containing complexes by BN-PAGE indicates that the presence of Mio10 complexes is independent of the $\mathrm{F}_{1} \mathrm{~F}_{\mathrm{O}} \mathrm{ATPase}$.

\subsubsection{Mio10 and MINOS1 are not stably associated with the $F_{1} F_{O} A T P a s e$}

In order to further investigate a possible Mio10 association with the $\mathrm{F}_{1} \mathrm{~F}_{\mathrm{O}} \mathrm{ATPase}$, a tagged Atp20 strain (Atp20 $0^{\mathrm{zz}}$ ) was used to biochemically isolate the $\mathrm{F}_{1} \mathrm{~F}_{\mathrm{O}} \mathrm{ATPase}$ and its associated components. Indeed, immunoprecipitation was successful for $\mathrm{F}_{1} \mathrm{~F}_{\mathrm{O}} \mathrm{ATPase}$ components such as Atp5, proving the technical success of the approach. 
However, Mio10 was not coisolated. These isolations were repeated using a streptavidin-flag C-terminally tagged Atp5 or Atp20 and with coimmunoprecipitations with Mio10 specific antibodies (data not shown). In all cases, Mio10 was not coisolated with the $\mathrm{F}_{1} \mathrm{~F}_{\mathrm{O}} \mathrm{ATPase}$ components. As solubilization buffer conditions and salt concentrations used in biochemical protein isolations from yeast differed from those used in BN-PAGE assembly experiments, it was taken into account that a higher salt concentration used in biochemical isolations may explain the lack of stable Mio10 association with the $\mathrm{F}_{1} \mathrm{~F}_{\mathrm{O}}$ ATPase. To compensate for these changes, immunoprecipitation with the Atp20 $20^{\mathrm{zz}}$ tagged strain was repeated with $\mathrm{BN}$ PAGE conditions. However, Mio10 was not coisolated with $\mathrm{F}_{1} \mathrm{~F}_{\mathrm{O}} \mathrm{ATP}$ ase subunits under these conditions (data not shown). In addition, the $\mathrm{F}_{1} \mathrm{~F}_{\mathrm{O}} \mathrm{ATPase}$ and its associated components were co-immunoprecipitated from HEK29T3 isolated mitochondria using $\mathrm{F}_{1} \mathrm{~F}_{\mathrm{O}} \mathrm{ATPase}$ specific antibodies. MINOS1 was not found to associate with the $\mathrm{F}_{1} \mathrm{~F}_{\mathrm{O}} \mathrm{ATPase}$ whereas the approach was successful in isolating Atp2. Thus, the biochemical isolations from yeast and human isolated mitochondria indicate that neither Mio10 nor MINOS1 are stably associated with the $\mathrm{F}_{1} \mathrm{~F}_{\mathrm{O}}$ ATPase and thus may not have a role in its dimerization.

\subsubsection{Mio10 does not affect $F_{1} F_{O} A T P a s e$ activity or oligomerization}

Analysis of the growth behavior of the $\mathrm{F}_{1} \mathrm{~F}_{\mathrm{O}} \mathrm{ATPase}$ dimerization factors indicates that they are not essential for mitochondrial respiration or $\mathrm{F}_{1} \mathrm{~F}_{\mathrm{O}} \mathrm{ATPase}$ activity, (Arnold et al., 1998; Paumard et al., 2002a; Soubannier et al., 2002). To assess the any effect a MIO10 deletion may have on mitochondrial respiration, yeast mio10د cells were grown in fermentable and non-fermentable media. In fact, miol0s cells continue to grow on non-fermentable medium and have a similar growth profile as atp20 cells. Mitochondrial respiration does not seem to be compromised in miol0s mutants in comparison with atp $2 \Delta$ cells. Atp2 is the beta subunit of the $\mathrm{F}_{1} \mathrm{~F}_{\mathrm{O}}$ ATPase and together with Atp1 form the catalytic core in the $F_{1}$ component (Takeda et al., 1985; Abrahams et al., 1994). In addition, an in-gel activity assay was used to assess the effect of MIO10 deletion on the activity of various respiratory chain complexes. Absence of Mio10 does not affect the enzymatic activity of $\mathrm{F}_{1} \mathrm{~F}_{\mathrm{O}} \mathrm{ATPase}$, cytochrome $c$ oxidase, or complex I. Therefore, it is concluded that Mio10 is not involved in $\mathrm{F}_{1} \mathrm{~F}_{\mathrm{O}} \mathrm{ATPase}$ activity or mitochondrial respiration. 
Using the miol0s strain, a possible role for Miol0 in $\mathrm{F}_{1} \mathrm{~F}_{\mathrm{O}} \mathrm{ATPase}$ dimerization was further assessed by BN-PAGE and Western blot analysis. Immunodetection for Atp5, which allowed the detection of $F_{1} F_{O} A T P a s e$ complexes, indicated the presence of intact $\mathrm{F}_{1} \mathrm{~F}_{\mathrm{O}} \mathrm{ATPase}$ dimers. Thus the absence of Mio10 does not affect dimerization. Various inner membrane proteins such as Fcj1 and its $C$. elegans homolog, IMMT-2, do not play a role in dimerization but affect the stability of the $\mathrm{F}_{1} \mathrm{~F}_{\mathrm{O}} \mathrm{ATPase}$ higher oligomers (Rabl et al., 2009; Mun et al., 2010). IMMT-2 was suggested to be involved in the formation of supermolecular complexes (Mun et al., 2010). On the other hand, FCJI overexpression reduces the stability $\mathrm{F}_{1} \mathrm{~F}_{\mathrm{O}} \mathrm{ATPase}$ due to its antagonistic effect on $\mathrm{F}_{1} \mathrm{~F}_{\mathrm{O}} \mathrm{ATPase}$ dimer mediated inner membrane organization (Rabl et al., 2009). The affect of Mio10 on oligomerization of the $\mathrm{F}_{1} \mathrm{~F}_{\mathrm{O}} \mathrm{ATPase}$ was assessed using the in-gel activity assay. In contrast to previous in-gel activity assays, digitonin concentrations were reduced to $0.35 \%$ and a $3-13 \%$ BN-PAGE gel gradient was used to better solubilize and separate larger complexes. The absence of Mio10 however does not negatively or positively affect $\mathrm{F}_{1} \mathrm{~F}_{\mathrm{O}} \mathrm{ATPase}$ oligomerization. Whether $\mathrm{MIO} 10$ overexpression has a similar effect on $\mathrm{F}_{1} \mathrm{~F}_{\mathrm{O}} \mathrm{ATPase}$ oligomers, as Fcj1, is yet to be determined.

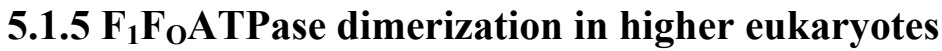

Analysis of the MIO10 deletion mutants indicated that Mio10 does not affect $\mathrm{F}_{1} \mathrm{~F}_{\mathrm{O}} \mathrm{ATPa}$ activity or its organization into dimers or higher oligomers. In conjunction with BN-PAGE analysis and biochemical isolations of tagged $\mathrm{F}_{1} \mathrm{~F}_{\mathrm{O}} \mathrm{ATPase}$ subunits, it is concluded that Mio10 and MINOS1 are not $\mathrm{F}_{1} \mathrm{~F}_{\mathrm{O}} \mathrm{ATPase}$ dimerization factors. This raises the question of what mediates $\mathrm{F}_{1} \mathrm{~F}_{\mathrm{O}} \mathrm{ATPase}$ dimerization in higher eukaryotes. It may be possible that different factors mediate dimerization across the species divide. The human IF1 protein promotes $\mathrm{F}_{1} \mathrm{~F}_{\mathrm{O}} \mathrm{ATPase}$ dimerization whereas its yeast homolog, Inh1, does not have a role (García et al., 2006). The observation that homologs of Atp18 and Atp19 are not found whereas homologs of Atp20 and Atp21 have no proven role in $\mathrm{F}_{1} \mathrm{~F}_{\mathrm{O}} \mathrm{ATPase}$ in higher eukaryotes may indeed suggest that small dimerization factors are not required in higher eukaryotes. Nonetheless, the in silico approach used in this study was not successful in identifying novel dimerization factors and perhaps different approaches are needed. One possibility would be affinity purification based mass spectrometric analysis of tagged $\mathrm{F}_{1} \mathrm{~F}_{\mathrm{O}} \mathrm{ATP}$ ase subunits. This may help identify novel components 
that may associate with the $\mathrm{F}_{1} \mathrm{~F}_{\mathrm{O}} \mathrm{ATPase}$ in higher eukaryotes. The question of what mediates $\mathrm{F}_{1} \mathrm{~F}_{\mathrm{O}} \mathrm{ATPase}$ dimerization in higher eukaryotes remains outstanding.

\subsection{The MINOS complex: Mitochondrial inner membrane organizing system complex}

\subsubsection{Identification of the yeast MINOS complex}

As initial analysis indicated that neither Mio10 nor its human homolog MINOS1 are $\mathrm{F}_{1} \mathrm{~F}_{\mathrm{O}} \mathrm{ATPase}$ dimerization factors, the question of Mio10/MINOS1 function still remained. Biochemical isolations from a Mio10 ${ }^{\mathrm{SF}}$ tagged strain were performed followed by mass spectrometric analysis to identify Mio10 interaction partners. Whereas, results further verified the lack of interaction with the $F_{1} F_{O} A T P a s e$, they indicated a possible Fcj1 interaction. This was also shown when Mio10 was successfully coisolated with Fcj1 from a Fcj1 ${ }^{\mathrm{zz}}$ strain. Fcj1 and its human homolog Mitofilin, play a major role in the organization of the inner membrane as mutants that lack Fcj1 or Mitofilin exhibit aberrant cristae morphologies (John et al., 2005; Rabl et al., 2009). This Mio10-Fcj1 interaction suggested that Mio10 could also be involved in inner membrane organization. In addition, many of the proteins that are found in the mass spectrometric analysis either uncharacterized and/or AIM proteins (Aim5, Aim13, Aim37, Aim38, Aim45). These proteins were found in screens that affect mitochondrial inheritance and may suggest other possible roles of Mio10 (Hess et al., 2009). However, due to the lack of antibodies against these proteins, it was difficult to verify some of the interactions. It should be noted that mass spectrometric analysis of Mio10 ${ }^{\mathrm{SF}}$ immunoprecipitation was not quantitative. The analysis was mainly based on spectral counts and normalized fold changes between elutions from tagged against wildtype untagged strains. Thus, it would be of interest to repeat the isolations with SILAC-based mass spectrometry, which might provide a more quantitative analysis that would further verify results of this study (Ong et al., 2002). Nonetheless, data indicated a Mio10 interaction with Fcj1, and therefore a possible similar role in cristae biogenesis.

A function for Mio10 in mitochondrial inner membrane organization was investigated using the mio $10 \Delta$ strain. Whereas mio $10 \Delta$ cells were not affected at $30^{\circ} \mathrm{C}$, cells lacking Mio10 showed a growth defect at $18^{\circ} \mathrm{C}$ and to lesser extent at $37^{\circ} \mathrm{C}$ when 
grown on minimal non-fermentable medium. A growth defect at elevated temperatures may indicate a change in membrane biogenesis and stability as shown in various mutants involved in cardiolipin biosynthesis (Joshi et al., 2009). Interestingly, $f_{c j} 1 \Delta$ cells had a similar growth behavior to mio10s cells. Upon further investigation of mitochondria isolated from miolos grown at $18^{\circ} \mathrm{C}$, no strong changes were observed at mitochondrial protein steady state levels. Live fluorescence microscopy was used to evaluate mitochondrial network organization of miol0s cells, which appeared to be more condensed compared to the wildtype strain. In fact, mio $10 \Delta$ and $f_{c j} 1 \Delta$ cells displayed very similar condensed mitochondrial networks, supporting the notion of a common Mio10/Fcj1 function. In contrast, atp200 mitochondria were fragmented, highlighting their different functional role in mitochondrial membrane organization. Mitochondrial dynamics (fusion/fission) provides a mechanism for compromised mitochondria to complement their damaged components with contents from healthy mitochondria (Ziviani and Whitworth, 2010). Indeed, the aberrant mitochondrial network dynamics observed in the examined mutant strains may be due to higher mitochondrial turnover. Mitochondria are degraded by mitophagy, which is the selective autophagy of mitochondria (Kanki et al., 2008). Previous studies have used an experimental system in which the mitochondrial outer membrane protein, Om45, is tagged with GFP and then used to monitor mitophagy (Kanki et al., 2009). A similar system thus can be applied to investigate mitophagy in miolos and $f_{c j} 1 \Delta$ cells.

On the other hand, analysis by electron microscopy revealed a strong aberration in the organization of the inner membrane when Mio10 was absent. The MIO10 and FCJ1 deletion mutants exhibited leaflet-like stacked cristae membranes compared to the wildtype. Also, both mutant strains appear to have lost defined cristae tips. The observed phenotype is in agreement to previous reports of $f_{c j} 1 \Delta$ mutants (Rabl et al., 2009). It is also clearly distinct from the onion-like organization observed in atp20د mitochondria and thus ruling out a common function (Paumard et al., 2002b). Previous work on $f c j 1 \Delta$ using tomography indicated that the observed stacked membranes have lost cristae junctions and are stacked by $F_{1} F_{O} A T P a s e$ dimer interactions between one membrane and another (Rabl et al., 2009). This may hold true for miol0s mitochondria, which have a remarkably similar inner membrane organization. All in all, the sum of data (biochemical isolations and MIO10 deletion 
mutants analysis) indicates that Mio10 is a novel interaction partner of Fcj1 that is also involved in mitochondrial inner membrane organization.

\subsubsection{Identification of the human MINOS complex}

In order to identify possible interaction partners of MINOS1 in human mitochondria, coimmunoprecipitation using MINOS1 specific antibodies were performed and isolated complexes were further analyzed. For this purpose, SILAC-based quantitative mass spectrometry analysis was performed, as well as a label-switch experiment. To this end, analysis of MINOS1 containing complexes from human isolated mitochondria indicated a significant enrichment of Mitofilin. Also, in agreement with previous studies, various Mitofilin interactors such as Metaxin 1, Metaxin 2, CHCHD3, HSPA9, DnaJC11, and SAM50 were also significantly enriched (Xie et al., 2007; Darshi et al., 2011; An et al., 2012). This finding highlights the efficiency of the experimental approach. The evidence of MINOS1 interaction with Mitofilin and CHCHD3 links MINOS1 with a role in cristae morphology. Results also verify the model that Mitofilin, which is located at cristae junctions effectively bridges the inner the outer mitochondrial membranes (Xie et al., 2007; Darshi et al., 2011). The MINOS1 interactions with Mitofilin, CHCHD3, and HSPA9 were verified by Western blot analysis. However, other interacting proteins could not be verified due to the unavailability of antibodies. Nonetheless, the results from the SILAC-based approach indicate that Mio10-Fcj1 interaction is conserved in higher eukaryotes.

In contrast to the enrichment of mitochondrial proteins, various nonmitochondrial proteins were also enriched. Enrichment of GDI1 (GDP Dissociation Inhibitor), which is involved in Rab GTPase cycle, may provide a link to vesicle transport and perhaps signaling between mitochondria and the extra-mitochondrial space (Bachner et al., 1995). Conversely, MIA3/TANGO1 is an endoplasmic reticulum (ER) integral membrane protein (Saito et al., 2009) and may implicate ER mitochondrial interactions. Indeed, an ER-mitochondria encounter structure (ERMES), which forms a junction between the two organelles, has been previously identified in yeast (Kornmann et al., 2009; Stroud et al., 2011). ERMES contains an ER-anchored Mmm1, and the mitochondrial proteins Gem1, Mdm10, Mdm12, and Mdm34 (Kornmann et al., 2009; Stroud et al., 2011). Coincidently, these ERMES components are also in involved mitochondrial maintenance and morphology (Okomoto and Shaw, 
2005). The observation that ERMES components interact with the SAM complex further indicates a possible involvement of MINOS with ERMES (Meisinger, et al., 2004, 2007). A role of ERMES in connecting mitochondria to the cytoskeleton has been also proposed (Buldogh et al., 2003). This can be exemplified by Miro, the homolog of Gem1 in higher eukaryotes, which can act as an adaptor between the two organelles (Glater et al., 2006; Koshiba et al., 2011). This interaction between mitochondria and components of the cytoskeleton plays an essential role in proper mitochondria movement and trafficking (Okamoto and Shaw, 2005). Interestingly, FAM82B (regulator of microtubule dynamics protein 1) and TUBG1 (Tubulin), which are also components of the microtubule cytoskeleton, are enriched in the SILAC based mass spectrometric analysis (Zheng et al., 1991; Oishi et al., 2007). Their interaction with MINOS1/Mitofilin may thus provide a link between inner membrane organization with mitochondrial-cytoskeleton interaction. Taken together, a MIA3 and SAM interaction with the mitochondrial inner membrane MINOS complex may thus provide a novel link between the mitochondrial inner compartment and the ER. Of course, more evidence with respect to these interactions is still required to be verified before any further speculations can be made.

\subsubsection{Mio10 and MINOS1 form a large complex (the MINOS complex)}

Mio10 and MINOS1 containing complexes were analyzed by size exclusion chromatography. Both Mio10 and MINOS1 were found to form large complexes in the MDa range. These complexes have a similar size to complexes that contain Fcj1 and Mitofilin. This is in agreement with previous reports that described Mitofilin to form a large mitochondrial complex (John et al., 2005). Taken together, in conjunction with topological and mass spectrometric analyses in yeast and human cells, results indicate that Mio10/MINOS1 form a large complex at the mitochondrial inner membrane. In human mitochondria, this complex may contain the known Mitofilin interactor CHCHD3 and bridges the mitochondrial membranes by interacting with the SAM complex. The exact composition of MINOS complex however cannot be determined from the results of this study. Moreover, this complex plays a role in cristae and inner membrane morphology, as shown by the MIO10 deletion mutants in yeast. The absence of this complex leads to aberrant inner membrane organization. Hence, this complex was termed MINOS: Mitochondrial Inner membrane Organizing System (Figure 35). Evidence of MINOS components in various species from yeast, 
C.elegans, and human mitochondria, as well as high sequence conservation of Mio10/MINOS1, Fcj1/Mitofilin, and CHCHD3 may reflect the functional and evolutionary importance of this complex.

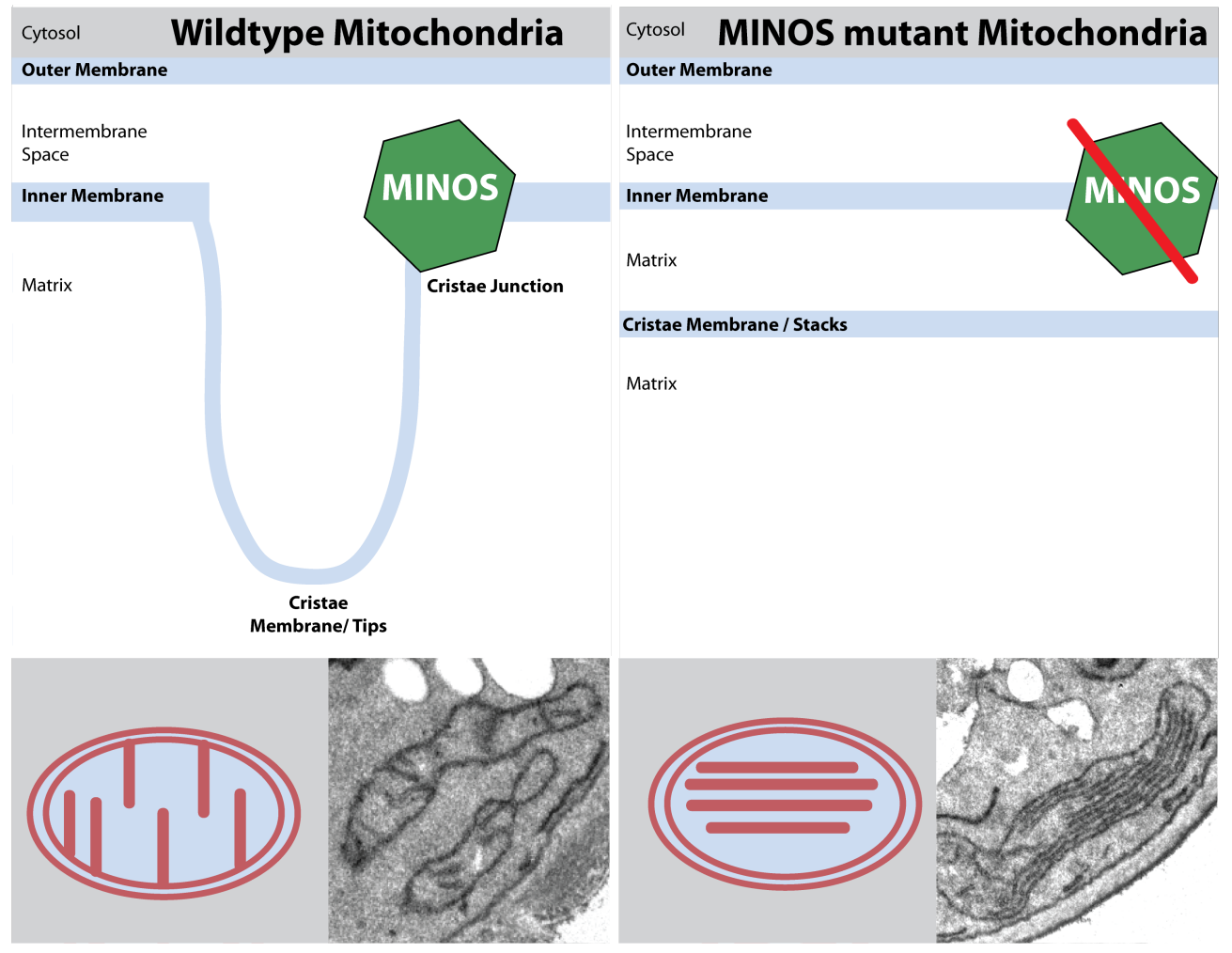

Figure 35. MINOS complex is critical for cristae biogenesis. In wildtype mitochondria, the MINOS complex sits at cristae junctions and contributes to proper cristae membrane morphology and cristae tip formation. In a MINOS mutant mitochondria (miolos or $f c j 1 \Delta$ ), the absence of the MINOS complex leads to aberrant cristae morphology exhibited by membrane stacks. Schematic representations of the phenotypes of wildtype and MINOS mutants (based on electron micrographs of mio10A) are shown the lower panel. 


\subsubsection{The identification of additional MINOS components}

During the revision process for the Alkhaja et al., 2012, three publications also reported the identification of the yeast MINOS complex (Hoppins et al., 2011; Harner et al., 2011; von der Malsburg et al., 2011). In all three studies, Mio10 (ORF, YCL057c-A) has been identified to play a role in cristae junction formation and inner membrane organization and morphology. This role is mediated by its interaction with Fcj1. Moreover, four other components of the MINOS complex have been also identified: YGR235C, Aim5, Aim13, and Aim37. Interestingly these proteins have been identified in the mass spectrometric analysis of Mio $10^{\mathrm{SF}}$ biochemical isolations, performed in this study. Von der Malsburg et al. identified the component the MINOS in an affinity purification screen using a Fcj1 ${ }^{\mathrm{zz}}$ strain coupled with SILAC-based quantitative mass spectrometric analysis, whereas Harner et al. identified the components by analyzing the proteome of contact sites. These sites are regions at which the outer membrane and inner membrane appear to meet (Hackenbrock, 1968). In contrast, Hoppins et al. identified the MINOS complex using a quantitative genetic interaction map. The systematic map was based on pair-wise measurements of growth defects in double mutant cells, and focused on genes encoding mitochondrial proteins and genes that may affect mitochondrial function. The components were found in a highly correlated cluster containing Fcj1 and also to a cluster containing ERMES. Like Mio10, these identified components affect inner membrane organization and cristae morphology, however with varying degrees. Table 8 represents the components of MINOS based on the different studies in comparison to available data with respect to higher eukaryotes. The following section reviews the findings of the three independent studies, in which the nomenclature used in der Malsburg et al. (2011) is applied. 
Table 8. The MINOS components as identified by various studies. MINOS, von der Malsburg et al., 2011; MICOS, Harner et al., 2011; MitOS, Hoppins et al., 2011. $\mathrm{n} / \mathrm{a}$, not available homolog in respective organism(s). YGR235C = (Mio27/Msc29/Mos2)

\begin{tabular}{|l|l|l|l|}
\hline \multicolumn{3}{|c|}{ Yeast } & $\begin{array}{l}\text { Higher } \\
\text { eukaryotes }\end{array}$ \\
\hline MINOS & MICOS & MitOS & MINOS \\
\hline Mio10 & Msc10 & Mos1 & MINOS1 \\
\hline Fcj1 & Fcj1 & Fcj1 & Mitofilin / IMMT \\
\hline Aim5 & Msc12 & Aim5 & $n / a$ \\
\hline Aim13 & Msc19 & Aim13 & $n / a$ \\
\hline Aim37 & Msc27 & Aim37 & MOMA-1 \\
\cline { 1 - 2 } Mio27 & Msc29 & Mos2 & \\
\cline { 2 - 3 } n/a & $n / a$ & $n / a$ & CHCHD3 \\
\cline { 1 - 2 } & & &
\end{tabular}

Topological analysis indicated that all identified components, besides Aim13, were integral inner membrane proteins with domains exposed to the IMS. Aim13 was shown to an IMS protein that associated peripherally to the inner membrane. Electron microscopy analysis of the different mutants indicated that miol0s and $f c j 1 \Delta$ had similar phenotypes in which mitochondria exhibited stacks of lamellar cristae. The observed phenotypes are in agreement with electron microscopy analysis performed in this doctoral study. Also, whereas mio27 4 mitochondrial had no considerable defects,

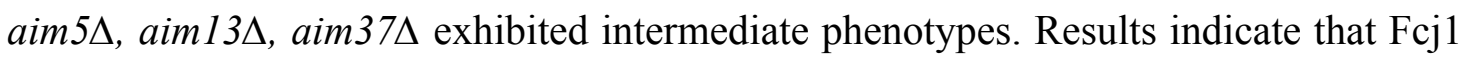
and Mio10 are the main functional components of the MINOS complex. The conclusion that cristae membranes loose their association with the IBM is $f c j 1 \Delta$ and mio10د is also made (von der Malsburg et al., 2011).

In agreement with results presented in this study, the MINOS complex was also shown to interact with the TOM and SAM complexes of the mitochondrial outer membrane (von der Malsburg et al., 2011, Harner et al., 2011). A MINOS interaction with Mia40 was also observed (von der Malsburg et al., 2011). The Mia pathway with its components Mia40 and Erv1 mediate oxidative protein folding by disulfide bond formation, which drive translocation and proper targeting of a certain mitochondrial IMS proteins (Herrmann and Riemer, 2012). However, an interaction with Mia40 was argued to be specific to Fcj1 and not other MINOS components. Fcj1 thus may have distinct functions in either inner membrane organization or protein import. Moreover, it is shown that Fcj1 copurifies with Ugo1, which is required for mitochondrial fusion 
in concert with its interaction partner Fzo1 (Sesaki and Jensen, 2004; Hoppins et al., 2011). The data thus not only links the MINOS complex with mitochondrial protein import (TOM, SAM) but also to the mitochondrial network dynamics. This also parallels results of this doctoral study which indicated potential interactions with the SAM complex and also ERMES components. All in all, the results of the recent studies are in agreement with the findings of this doctoral study and support the model of the MINOS complex involvement in inner membrane organization.

\subsection{Conclusions and Outlook}

The mitochondrial inner membrane is morphologically dynamic. Various inner membrane proteins are known to contribute to the shape and structure of cristae membranes. $\mathrm{F}_{1} \mathrm{~F}_{\mathrm{O}} \mathrm{ATPase}$ dimers contribute to a strong local curvature and thus the angular shape of the cristae apex (Strauss et al., 2008; Seelert and Dencher, 2011). Mutants that lack the Atp20 or Atp21 dimerization factors exemplify the importance of $\mathrm{F}_{1} \mathrm{~F}_{\mathrm{O}} \mathrm{ATPase}$ dimerization with respect to cristae morphology. These mutants clearly exhibit mutant inner mitochondrial organization that characterized by concentric 'onion-like' structures (Paumard et al., 2002b; Arselin et al., 2003; Arselin et al., 2004). Moreover, Mitofilins (Fcj1 in yeast) has been previously shown to be an important determinant of cristae junction formation by acting in an antagonistic manner in inner membrane curvature with respect to $\mathrm{F}_{1} \mathrm{~F}_{\mathrm{O}} \mathrm{ATPase}$ dimers (Rabl et al., 2009). Whereas this study did not result the identification of novel $F_{1} F_{O} A T P a s e$ dimerization factors, Mio10/MINOS1 are identified as novel interaction partners of Fcj1/Mitofilin. This study also provided evidence for the MINOS complex playing a critical role in inner membrane architecture. The role of Mio10 in cristae morphology in yeast was shown by the analysis of MIO10 deletion mutants in comparison to other strains. The results of this study is paralleled and verified by three other studies, which have in addition identified four other components of the MINOS complex (Hoppins et al., 2011; Harner et al., 2011; von der Malsburg et al., 2011). Comparison of the phenotypes of the various MINOS mutants, indicated that the deletion of MIOIO and FCJ1 lead to more severe cristae aberrations. The absence of either protein also leads to complete dissociation of the MINOS complex in yeast (von der Malsburg et al., 2011). It thus appears that Mio10 is one the main components of the yeast MINOS complex. 
Results from this study and other publications support the notion that MINOS is a multifunctional complex (Figure 36). Beside the obvious role in cristae morphology, the MINOS may play a role in mitochondrial protein import by its interaction with SAM, TOM, and MIA components (Xie et al., 2007; von der Malsburg et al., 2011; Harner et al., 2011). By its interaction with Ugo1, the MINOS complex plays a role in fusion and mitochondrial network dynamics (Harner et al., 2011). Moreover, it may have a role in mitochondrial inheritance via its components, Aim5, Aim13, and Aim37 (Hess et al., 2009). MINOS interaction with cytoskeleton proteins (FAM82B and Tubulin) may indicate its role in mitochondrial trafficking. Furthermore it may play a role in ERMES and ER mitochondrial dynamics via its interaction with ER resident proteins (TANGO1). This notion is further supported by a strong genetic interaction of MINOS and ERMES genes (Hoppins et al., 2011). Besides its link to apoptosis via Mitofilin interaction with OPA1 (Darshi et al., 2011), the MINOS complex may also play role in cancer. Indeed, MINOS1 proteins levels are elevated in prostate cancer cells (Lin et al., 2005). In addition, overexpression of CHCHD6 in human cancer cells enhances resistance to genotoxic anticancer drugs, whereas its knockdown increases its chemosensitivity (An et al., 2012). Other than its proven role in inner membrane architecture, any role MINOS might have in other mitochondrial and non mitochondrial processes remains speculative and further investigations are necessary. 


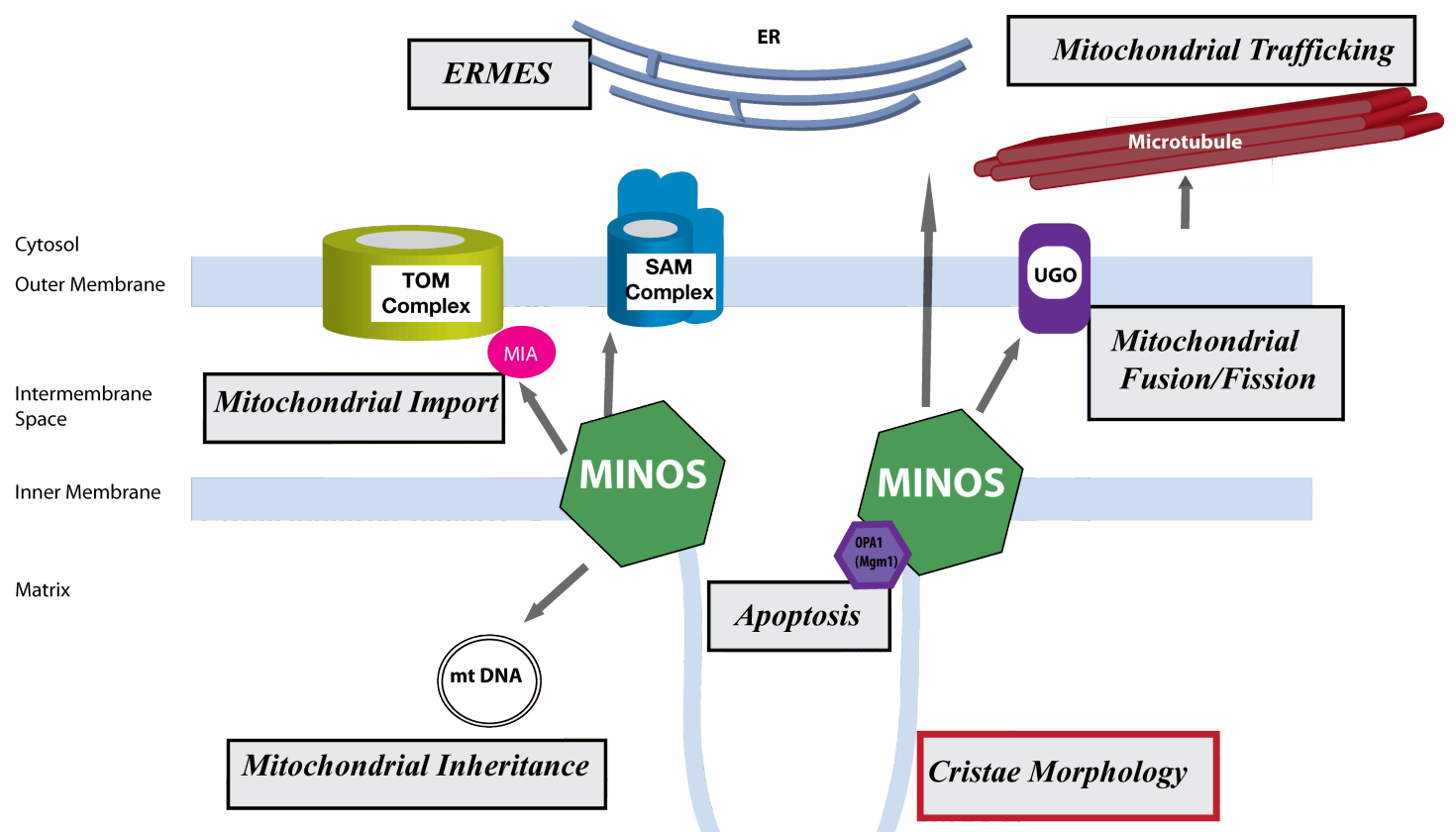

Figure 36. MINOS is a multifunctional protein. Besides the MINOS role in cristae morphology, results from various sources indicate potential MINOS interactions with ERMES (via SAM and TANGO1), mitochondrial trafficking (Tubulin), mitochondrial import (TOM, SAM, Mia40), mitochondrial fusion/fission (Ugo1), and mitochondrial inheritance (AIM proteins of the MINOS complex).

Besides the characterization of novel roles MINOS may have in mitochondria and other cellular organelles, information is still required with respect to the structure and exact function of MINOS in inner membrane organization. For instance, the exact composition of the MINOS complex in higher eukaryotes remains an open question. Still, it may be postulated from available data that MINOS in higher eukaryotes contains at least MINOS1, Mitofilin, CHCHD3 and CHCHD6. It should also be considered that the composition of the MINOS complex might vary across the species divide. A homolog of Aim5 and Aim13 is not found in higher eukaryotes whereas a homolog for CHCHD3 is not found in yeast (Herrmann, 2011). The MINOS complex may require at least Mio10/MINOS1 and Fcj1/Mitofilin, whereas other components may vary. One possible approach would be performing an affinity purification based mass spectrometric analysis of a tagged MINOS1 or Mitofilin in human cultured cells. This would be comparable to the approach in which all MINOS components were identified in yeast (von der Malsburg et al., 2011). In addition, the effect of the roles of Mitofilin, CHCHD3, and CHCHD6 are well documented as their depletion leads to aberrant mitochondrial cristae (John et al., 2005; Darshi et al., 2011; An et al., 2012). To this end, it would be valuable to investigate the effect of MINOS1 knockdown by 
RNAi in human mitochondria and whether the role of MINOS1 in the MINOS complex is as essential as the yeast Mio10.

More evidence is still required to understand how the MINOS complex and its components exert their function in cristae biogenesis. It should be noted that most of the of evidence related to the roles of these inner membrane proteins have been deduced from tomographies of mutants, whether yeast or human cultured cells. These tomographies represent single-point illustrations of the mutations effects but provide limited insight onto the dynamics governing how cristae membranes are rendered 'online-like', 'stacked', or 'vesicle-like'. This further highlights the extent to which cristae propagation, maintenance, and dynamics remain to be studied.

Analysis of the MINOS mutants indicted that that MINOS complex affects cristae junction opening diameters (Rabl et al., 2009; von der Malsburg et al., 2011; Hoppins et al., 2011). It also plays a role in maintenance of cristae tips, exemplified by the lack of cristae tips in various MINOS mutants. In the absence of MINOS components, the cristae membranes appear detached from the IBM and form stacks that are linked together with the $\mathrm{F}_{1} \mathrm{~F}_{\mathrm{O}} \mathrm{ATPa}$ (Rabl et al., 2009; von der Malsburg et al., 2011). MINOS might thus have a role in cristae biogenesis: cristae tip formation, cristae membrane curvature, junction maintenance; and cristae membrane propagation (Figure 37). 


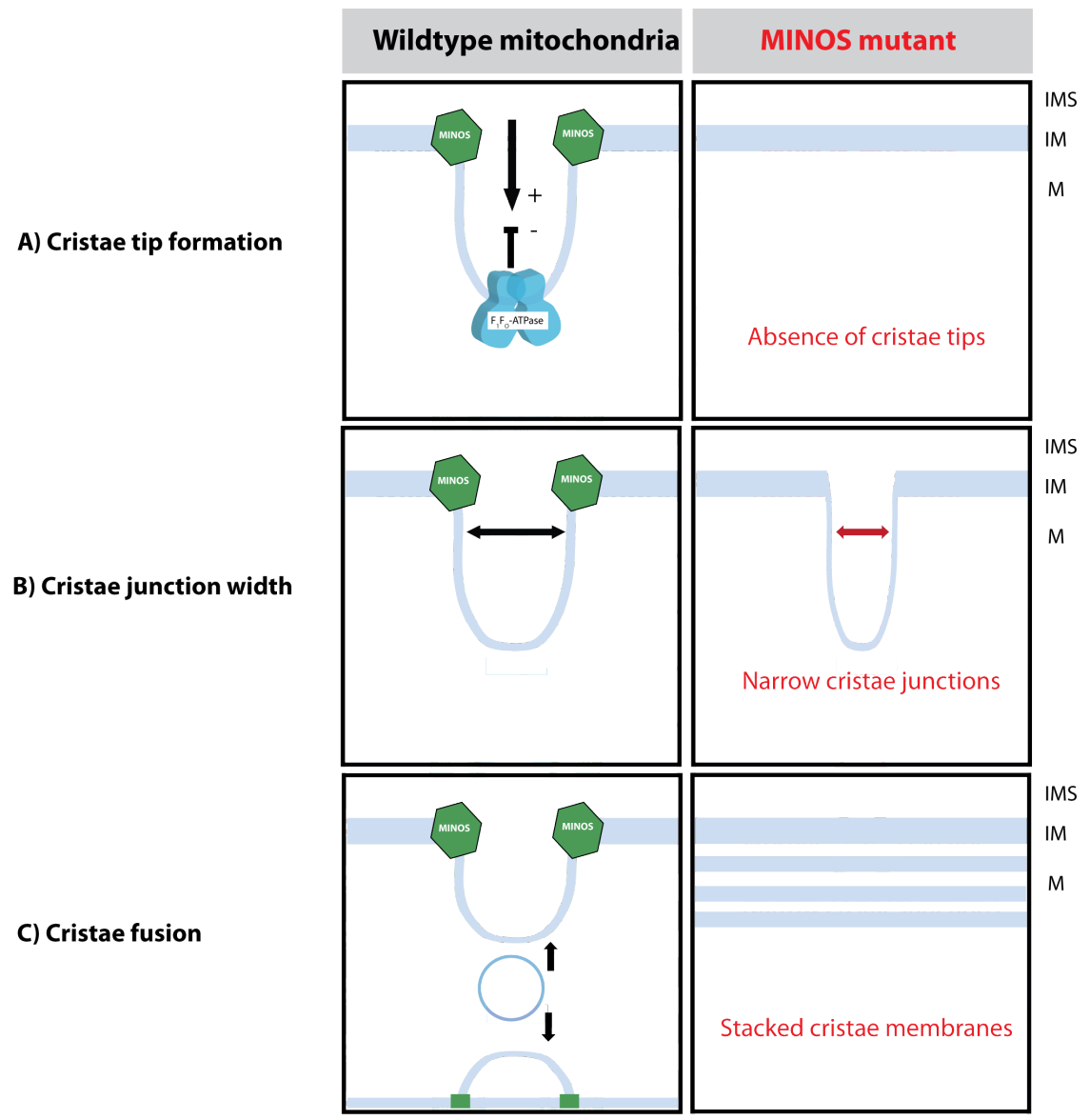

Figure 37. Overview of MINOS roles in cristae biogenesis. A) By inducing positive membrane curvature, MINOS leads to invagination of the inner membrane and eventual cristae tip formation. In the absence of MINOS, cristae tips are not found. B) The large molecular size of MINOS might regulate cristae junction width. In the absence of some MINOS components, cristae membranes appear to have narrower cristae junctions. C) MINOS might have a role in the regulation cristae fusion. In the absence of MINOS, fission of cristae membrane is active whereas fusion discontinues. This may lead to an increase in cristae vesicle, which are then stacked by $\mathrm{F}_{1} \mathrm{~F}_{\mathrm{O}} \mathrm{ATPase}$ dimers.

MINOS may affect cristae junction due its large molecular size and by its interaction with OPA-1. OPA-1 controls cristae opening size by forming a scaffold at the opening that prevents premature release of cytochrome c under non-apoptotic conditions (Frezza et al., 2006). The role of MINOS in cristae biogenesis may be explained by the invagination cristae model, which postulates that cristae tips and membranes can result from 1) continuous inner membrane biogenesis and but constant outer membrane growth and 2) large complexes that enforce membrane curvature (Renken et al., 2002). The cristae membrane curvatures are mediated by the large MINOS complex (that forms a positive membrane curvature) and the $\mathrm{F}_{1} \mathrm{~F}_{\mathrm{O}} \mathrm{ATPase}$ dimers (which form a negative curvature). Thus, in the absence of MINOS, positive membrane curvature is absent and no cristae tips are formed. However, this may be not 
enough to explain the observed stacked cristae inner membranes of MINOS mutants. In this case, a cristae fusion/fission model may be applied. This model proposes that cristae formation is dynamic, in which cristae membrane vesicles may continuously fuse from one site of the inner membrane and fuse to another (Zick et al., 2009). The notion that cristae propagation is dynamic is supported by the observation of certain reversible cristae morphologies (Mannella et al., 2001). Stacked membranes in MINOS mutants may thus be due to continuous cristae fission but a lack of fusion. The MINOS complex may play a role in the equilibrium between cristae fusion and fission. Information is still lacking to explain a variation of phenotypes within any single MINOS mutants (wildtype, onion-like, or intermediate). Thus, it may seem that other factors may be involved with respect to MINOS and cristae morphology. Further analysis such as the analysis of multiple deletions or knockouts of MINOS components may shine light on the exact role of MINOS. Moreover, high-resolution immunoelectron microscopy analysis of mitochondrial inner membrane in the presence or absence of MINOS components may provide novel insight into the mechanism of the complex.

MINOS is not only an inner membrane organizer that forms a scaffold connecting the inner and outer mitochondrial membranes, but may feature in various other mitochondrial and extra-mitochondrial functions. Many of these functions are yet to be examined and further studies are required. Various outstanding questions remain with respect to the exact role of the MINOS complex. Nonetheless, the discovery of new MINOS components here may provide new insight and direction for future studies. 


\section{REFERENCES}

Abdul, K.M., Terada, K., Yano, M., Ryan, M.T., Streimann, I., Hoogenraad, N.J. and Mori, M. (2000). Functional analysis of human metaxin in mitochondrial protein import in cultured cells and its relationship with the Tom complex. Biochem. Biophys. Res. Commun. 276, 1028-1034.

Abrahams J. P., Leslie, A. G., Lutter, R., and Walker, J. E. (1994). Structure at 2.8 A resolution of F1ATPase from bovine heart mitochondria. Nature. 370, 621-628.

Ahting, U., Thieffry, M., Engelhardt, H., Hegerl, R., Neupert, W., and Nussberger, S. (2001). Tom40, the pore-forming component of the protein-conducting TOM channel in the outer membrane of mitochondria. J. Cell Biol. 153, 1151-1160.

Alkhaja, A. K., Jans, D. C, Nikolov, M., Vukotic, M., Lytovchenko, O., Ludewig, F., Schliebs, W., Riedel, D., Urlaub, H., Jakobs, S., and Deckers M. (2012). MINOS1 is a conserved component of mitofilin complexes and required for mitochondrial function and cristae organization. Mol. Biol. Cell. 23, 247-57.

Allen, R.D., Schroeder, C.C., and Fok, A.K. (1989). An investigation of mitochondrial inner membranes by rapid-freeze deep-etch techniques, J. Cell Biol. 108, 2233-2240.

Althoff, T., Mills, D.J., Popot, J.-L., and Kühlbrandt, W. (2011). Arrangement of electron transport chain components in bovine mitochondrial supercomplex I(1)III(2)IV(1). EMBO J. 30, 4652-4664.

Amutha, B., Gordon, D. M., Gu, Y., and Pain, D. (2004). A novel role of Mgm1p, a dynamin-related GTPase, in ATP synthase assembly and cristae formation/ maintenance. Biochem. J. 381,19-23.

An, J., Shi, J., He, Q., Lui, K., Liu, Y., Huang, Y., and Sheikh MS. (2012). CHCM1/CHCHD6, a novel mitochondrial protein linked to regulation of mitofilin and mitochondrial cristae morphology. J. Biol. Chem. [Epub ahead of print]

Andersson, S. G., Karlberg, O., Canback, B., and Kurland, C. G. (2003) On the origin of mitochondria: a genomics perspective. Philos. Trans. R. Soc. Lond. B. Biol. Sci. 358,165-177.

Arnold, I., Pfeiffer, K., Neupert, W., Stuart, R. A., and Schägger, H. (1998). Yeast mitochondrial F1F0ATP synthase exists as a dimer: identification of three dimer-specific subunits. EMBO J. 17, 71707178.

Arnold, I., Bauer, M. F., Brunner, M., Neupert, W., and Stuart, R. A. (1997). Yeast mitochondrial F1F0ATPase: the novel subunit e is identical to Tim11. FEBS Lett. 411, 195-200.

Arnold, I., Pfeiffer, K., Neupert, W., Stuart, R. A., and Schägger, H. (1998). Yeast mitochondrial F1F0ATP synthase exists as a dimer: identification of three dimer-specific subunits. EMBO J. 17, 71707178.

Arnold, I., Pfeiffer, K., Neupert, W., Stuart, R. A., and Schägger, H. (1999). ATP synthase of yeast mitochondria. Isolation of subunit $\mathrm{j}$ and disruption of the ATP18 gene. J. Biol. Chem. 274, 36-40.

Arselin, G., Giraud, M. F., Dautant, A., Vaillier, J., Brèthes, D., Coulary-Salin, B., Schaeffer, J., and Velours, J. (2003). The GxxxG motif of the transmembrane domain of subunit e is involved in the dimerization/oligomerization of the yeast ATP synthase complex in the mitochondrial membrane. Eur. J. Biochem. 270, 1875-1884.

Arselin, G., Vaillier, J., Salin, B., Schaeffer, J., Giraud, M. F., Dautant, A., Brèthes, D., and Velours, J. (2004). The modulation in subunits e and $g$ amounts of yeast ATP synthase modifies mitochondrial cristae morphology. J. Biol. Chem. 279, 40392-40399. 
Bachner, D., Sedlacek, Z., Korn B., Hameister H., and Poustka, A. (1995). Expression patterns of two human genes coding for different rab GDP-disso-ciation inhibitors (GDIs), extremely conserved proteins involved in cellular transport. Hum. Mol. Genet. 4, 701-708.

Banci, L., Bertini, I., Cefaro, C., Ciofi-Baffoni, S., Gallo, A., Martinelli, M., Sid-eris, D.P., Katrakili, N., and Tokatlidis, K. (2009). MIA40 is an oxidoreductase that catalyzes oxidative protein folding in mitochondria. Nat. Struct. Mol. Biol. 16, 198-206.

Becker, L., et al. (2005). Preprotein translocase of the outer mitochondrial membrane: reconstituted Tom40 forms a characteristic TOM pore. J. Mol. Biol. 353, 1011-20.

Bihlmaier, K., Mesecke, N., Terziyska, N., Bien, M., Hell, K., and Herrmann, J.M. (2007). The disulfide relay system of mitochondria is connected to the respiratory chain. J. Cell Biol. 179, 389-395.

Bradford, M. M. (1976). A rapid and sensitive method for the quantitation of microgram quantities of protein utilizing the principle of protein-dye binding. Anal Biochem. 72, 248-54.

Brandner, K., Mick, D. U., Frazier, A. E., Taylor, R. D., Meisinger, C., and Rehling, P. (2005). Taz1, an outer mitochondrial membrane protein, affects stability and assembly of inner membrane protein complexes: implications for Barth Syndrome. Mol Biol Cell. 2005. 11, 5202-5214.

Baughman, J. M., Nilsson, R., Gohil, V. M., Arlow, D. H., Gauhar, Z., and Mootha, V. K. (2009). A computational screen for regulators of oxidative phosphorylation implicates SLIRP in mitochondrial RNA homeostasis. 5, e1000590

Bornhövd, C., Vogel, F., Neupert, W., and Reichert, A. (2006). Mitochondrial membrane potential is dependent on oligomeric state of F1F0-ATP synthase supracomplexes. J. Biol. Chem. 281, 1399013998.

Boyer, P. D. (1997). The ATP synthase--a splendid molecular machine. Annu. Rev. Biochem. 66, 717749.

Boldogh, I. R., Nowakowski, D. W., Yang, H. C., Chung, H., Karmon, S., Royes, P., and Pon, L. A. (2003). A protein complex containing Mdm10p, Mdm12p, and Mmm1p links mitochondrial membranes and DNA to the cytoskeleton-based segregation machinery. Mol. Biol. Cell. 14, 4618-4627.

Burton, P.R. (1979). Fine structure of mitochondria of Spirostomum ambiguum as seen in sectioned and negatively-stained preparations. J. Protozool. 17, 295-299.

Bustos, D. M., and Velours, J. (2005). The modification of the conserved GXXXG motif of the membrane-spanning segment of subunit $g$ destabilizes the supramolecular species of yeast ATP synthase. J. Biol. Chem. 280, 29004-29010.

Buzhynskyy, N., Sens, P., Prima, V., Sturgis, J. N., and Scheuring, S. (2007). Rows of ATP synthase dimers in native mitochondrial inner membranes. 8, 2870-2876.

Campanella, M., Parker, N., Tan, C.H., Hall, A.M., and Duchen, M.R. (2009). IF1: setting the pace of the F1FO-ATP synthase, Trends. Biochem. Sci. 34, 343-350.

Chacinska, A., Lind, M., Frazier. A. E., Dudek, J., Meisinger, C., Geissler, A., Sickmann, A., Meyer, H. E., Truscott, K. N., Guiard, B., Pfanner, N., and Rehling, P. (2005). Mitochondrial presequence translocase: switching between TOM tethering and motor recruitment involves Tim21 and Tim17. Cell. $120,817-29$.

Chacinska, A., Koehler, C.M., Milenkovic, D., Lithgow, T., and Pfanner, N. (2009). Importing Mitochondrial Proteins: Machineries and Mechanisms. Cell. 4, 628-644.

Chenna, R., Sugawara, H., Koike, T., Lopez, R., Gibson, T. J., Higgins D, G., and Thompson, J. D. (2003). Multiple sequence alignment with the Clustal series of programs. Nucleic Acids Res. 31, 3497- 
3500.

Chernomordik, L. V., and Kozlov, M. M. (2005). Membrane hemifusion: crossing a chasm in two leaps. Cell. 123, 375-82.

Collinson, I. R., Runswick, M. J., Buchanan, S. K., Fearnley, I. M., Skehel, J. M., van Raaij, M. J., Griffiths, D. E., and Walker, J. E. (1994). Fo membrane domain of ATP synthase from bovine heart mitochondria: purification, subunit composition, and reconstitution with F1-ATPase. Biochemistry. 33, 7971-7978.

Cox, J., and Mann, M. (2008). MaxQuant enables high peptide identification rates, individualized p.p.b.range mass accuracies and proteome-wide protein quantification. Nat. Biotechnol. 26, 1367-1372.

Curran, S. P., Leuenberger, D., Oppliger, W., and Koehler, C. M. (2002). The Tim9p-Tim10p complex binds to the transmembrane domains of the ADP/ATP carrier. EMBO J. 21, 942-53.

Darshi, M., Mendiola, V. L., Mackey, M. R., Murphy, A. N., Koller, A., Perkins, G. A., Ellisman, M. H., and Taylor, S. S. (2011). ChChd3, an inner mitochondrial membrane protein, is essential for maintaining crista integrity and mitochondrial function. J. Biol. Chem. 286, 2918-2932.

De los Rios Castillo, D., Zarco-Zavala, M., Olvera-Sanchez, S., Pardo, J. P., Juarez, O., Martinez, F., Mendoza-Hernandez, G., García-Trejo, J. J., and Flores-Herrera, O. (2011). Atypical cristae morphology of human syncytiotrophoblast mitochondria: role for complex V. J. Biol. Chem. 286, 23911-23919.

Dian, E.A., Papatheodorou, P., Emmrich, K., Randel, O., Geissler, A., Kolling, R.,

Rassow, J., and Motz, C. (2008). Role of gamma-subunit N- and C-termini in assembly of the mitochondrial ATP synthase in yeast. J. Mol. Biol. 377,1314-1323.

Dolezal, P., Likic, V., Tachezy. J., and Lithgow, T. (2006). Evolution of the molecular machines for protein import into mitochondria. Science. 313, 314-318.

Duvezin-Caubet, S., Jagasia, R., Wagener, J., Hofmann, S., Trifunovic, A., Hansson, A., Chomyn, A., Bauer, M. F., Attardi, G., Larsson, N. G., Neupert, W., and Reichert, A.S. (2006). Proteolytic processing of OPA1 links mitochondrial dysfunction to alterations in mitochondrial morphology. J. Biol. Chem. 281, 37972-37979.

Dudkina, N. V., Sunderhaus, S., Braun, H. P., and Boekema, E. J. (2006). Characterization of dimeric ATP synthase and cristae membrane ultrastructure from Saccharomyces and Polytomella mitochondria. FEBS Lett. 580, 3427-3432

Egner, A., Jakobs, S., and Hell, S. W. (2002) Fast 100-nm resolution three-dimensional microscope reveals structural plasticity of mitochondria in live yeast. Proc Natl. Acad. Sci. U S A. 99, 3370-3375.

Erdmann, R., Veenhuis, M., Mertens, D., and Kunau, W. H. (1989). Isolation of peroxisome-deficient mutants of Saccharomyces cerevisiae. Proc. Natl. Acad. Sci. USA. 86, 5419-5423.

Eubel, H., Jänsch, L., and Braun, H. P. (2003). New insights into the respiratory chain of plant mitochondria. Supercomplexes and a unique composition of complex II. Plant Physiol. 133, 274-286.

Everard-Gigot, V., Dunn, C. D., Dolan, B. M., Brunner, S., Jensen, R. E., and Stuart, R.A. (2005). Functional analysis of subunit e of the F1FO-ATP synthase of the yeast Saccharomyces cerevisiae: importance of the N-terminal membrane anchor region. Eukaryot. Cell. 4, 346-355.

Farrell, S.R., and Thorpe, C. (2005). Augmenter of liver regeneration: a flavin- dependent sulfhydryl oxidase with cytochrome c reductase activity. Biochemistry. 44, 1532-1541.

Frazier, A. E, Chacinska, A., Truscott, K. N, Guiard, B., Pfanner, N., and Rehling, P. (2003).

Mitochondria use different mechanisms for transport of multispanning membrane proteins through the intermembrane space. Mol. Cell. Biol. 23, 7818-28. 
Frey, T.G., and Mannella, C.A. (2000). The internal structure of mitochondria. Trends Biochem. Sci. 25, 319-324.

Frezza, C. et al. (2006). OPA1 controls apoptotic cristae remodeling independently from mitochondrial fusion. Cell. 126, 177-189.

Fronzes, R., Chaignepain, S., Bathany, K., Giraud, M. F., Arselin, G., Schmitter, J. M., Dautant, A., Velours, J., and Brèthes D. (2003). Topological and functional study of subunit h of the F1Fo ATP synthase complex in yeast Saccharomyces cerevisiae. Biochemistry. 42, 12038-12049.

Fontanesi, F., Soto, I. C., Horn, D., and Barrientos, A. (2006) Assembly of mitochondrial cytochrome c-oxidase, a complicated and highly regulated cellular process. Am J Physiol Cell Physiol, 291, 1129-1147.

García, J. J., Morales-Ríos, E., Cortés-Hernandez, P., and Rodríguez-Zavala, J. S. (2006). The inhibitor protein (IF1) promotes dimerization of the mitochondrial F1F0-ATP synthase. Biochemistry. 42, $12695-12703$.

Gavin, P. D., Prescott, M., Luff, S. E., and Devenish, R. J. (2004). Cross-linking ATP synthase complexes in vivo eliminates mitochondrial cristae. J. Cell. Sci. 117, 2333-2343.

Geissler, A., Chacinska, A., Truscott, K. N., Wiedemann, N., Brandner, K., Sickmann, A., Meyer, H. E., Meisinger, C., Pfanner, N., and Rehling P. (2002). The mitochondrial presequence translocase: an essential role of Tim50 in directing preproteins to the import channel. Cell. 111, 507-518.

Giddings, T. H. (2003). Freeze-substitution protocols for improved visualization of membranes in highpressure frozen samples. J. Microsc. 212, 53-61.

Giraud, M. F., Paumard, P., Soubannier, V., Vaillier, J., Arselin, G., Salin, B., Schaeffer, J., Brèthes, D., di Rago, J. P., and Velours, J. (2002). Is there a relationship between the supramolecular organization of the mitochondrial ATP synthase and the formation of cristae? Biochim. Biophys. Acta. 1555, 174-180.

Gieffers, C., Korioth, F., Heimann, P., Ungermann, C., and Frey, J. (1997). Mitofilin is a transmembrane protein of the inner mitochondrial membrane expressed as two isoforms. Exp. Cell. Res. 232, 395-9.

Glater, E. E., Megeath, L. J., Stowers, R. S., and Schwarz, T.L. (2006). Axonal transport of mitochondria requires milton to recruit kinesin heavy chain and is light chain independent. J. Cell. Biol. $173,545-557$.

Glick, B. S., Brandt, A., Cunningham, K., Müller, S., Hallberg, R. L., and Schatz, G. (1992).

Cytochromes $\mathrm{c} 1$ and $\mathrm{b} 2$ are sorted to the intermembrane space of yeast mitochondria by a stop-transfer mechanism. Cell. 69, 809-822.

Gray, M.W., Burger, G., and Lang, B. F. (1999) Mitochondrial evolution. Nature. 283, 1476-1481.

Gray, M.W. (2011). The incredible shrinking organelle. EMBO Rep. 9, 873.

Griparic, L., van der Wel, N. N., Orozco, I.J., Peters, P.J., and van der Bliek, A.M. (2004). Loss of the intermembrane space protein Mgm1/OPA1 induces swelling and localized constrictions along the lengths of mitochondria. J Biol Chem. 279, 18792-18798.

Hackenbrock, C. R. (1968) Chemical and physical fixation of isolated mitochondria in low-energy and high-energy states. Proc. Natl. Acad. Sci. USA. 61, 598-605.

Harner, M., Körner, C., Walther, D., Mokranjac, D., Kaesmacher, J., Welsch, U., Griffith, J., Mann, M., Reggiori, F., and Neupert, W. (2011). The mitochondrial contact site complex, a determinant of mitochondrial architecture. EMBO J. 30, 4356-4370. 
Hess, D.C., et al. (2009). Computationally driven, quantitative experiments discover genes required for mitochondrial biogenesis. PLoS Genet. 5, e1000407.

Herrmann, J. M. (2011). MINOS is plus: a Mitofilin complex for mitochondrial membrane contacts. Dev Cell. 21, 599-600.

Herrmann, J. M., and Riemer, J. (2012). Mitochondria disulfide relay: a redox regulated protein import into the intermembrase space. J. Biol. Chem. 287, 4426-4433.

Hofmann, K. and Stoffel, W. (1993). TMBASE - a database of membrane spanning proteins segments. Biol. Chem. Hoppe-Seyler, 374, 166.

Holland, H. D. (1994) Early proterozoic atmospheric change. In Early life on earth (ed. S. Bengtson), pp. 237-244. New York: Columbia University Press.

Hong, S., and Pedersen, P. L. (2002). ATP synthase of yeast: structural insight into the different inhibitory potencies of two regulatory peptides and identification of a new potential regulator. Arch. Biochem. Biophys. 405, 38-43.

Hoppins, S., Lackner, L., and Nunnari, J. (2007). The machines that divide and fuse mitochondria. Annu. Rev. Biochem. 76, 751-780.

Hoppins, S., Collins, S. R., Cassidy-Stone, A., Hummel, E., Devay, R. M., Lackner, L. L, Westermann, B., Schuldiner, M., Weissman, J. S., and Nunnari, J. (2011). A mitochondrial-focused genetic interaction map reveals a scaffold-like complex required for inner membrane organization in mitochondria. J. Cell. Biol. 195, 323-40.

John, G. B., Shang, Y., Li, L., Renken, C., Mannella, C. A., Selker, J. M., Rangell, L., Bennett, M. J., and Zha, J. (2005). The mitochondrial inner membrane protein mitofilin controls cristae morphology. Mol. Biol. Cell. 16, 1543-1554.

Kanki, T., and Klionsky, D. J. (2008). Mitophagy in yeast occurs through a selective mechanism. J. Biol. Chem. 283, 32386-32393.

Kanki, T., Kang, D., and Klionsky, D. J. (2009). Monitoring mitophagy in yeast: the Om45-GFP processing assay. Autophagy. 5, 1186-1189.

Karlberg, O., Canbäck, B., Kurland, C.G, and Andersson, S.G. (2000). The dual origin of the yeast mitochondrial proteome. 3, 170-187.

Krause, F., Reifschneider, N. H., Goto, S., and Dencher, N. A. (2005). Active oligomeric ATP synthases in mammalian mitochondria. Biochem. Biophys. Res. Commun. 329, 583-590.

Kolmakov, K., Belov, V. N., Bierwagen, J., Ringemann, C., Müller, V., Eggeling, C., and Hell, S. W. (2010). Red-emitting rhodamine dyes for fluorescence microscopy and nanoscopy. Chemistry. 16, 158166.

Koshiba, T., Holman, H. A., Kubara, K., Yasukawa, K., Kawabata, S., Okamoto, K., MacFarlane, J., and Shaw, J. M. (2011). Structure-function analysis of the yeast mitochondrial Rho GTPase, Gemlp: implications for mitochondrial inheritance. J. Biol. Chem. 286, 354-362.

Kucharczyk, R., Zick, M., Bietenhader, M., Rak, M., Couplan, E., Blondel, M., Caubet, S.D., and di Rago, J.P. (2009). Mitochondrial ATP synthase disorders: molecular mechanisms and the quest for curative therapeutic approaches. Biochim. Biophys. Acta. 1, 186-99.

Lazarou, M., Smith, S. M., Thorburn, D. R., Ryan, M. T., and McKenzie. M. (2009). Assembly of nuclear DNA-encoded subunits into mitochondrial complex IV, and their preferential integration into supercomplex forms in patient mitochondria. FEBS J. 276, 6701-6713. 
Lin, B., et al. (2005). Evidence for the presence of disease-perturbed networks in prostate cancer cells by genomic and proteomic analyses: a systems approach to disease. Cancer. Res. 65, 3081-3091.

Liu, Q., D'Silva, P., Walter, W., Marszalek, J., and Craig, E. A. (2003). Regulated cycling of mitochondrial Hsp70 at the protein import channel. 300, 139-41.

Liu, H., Sadygov, R. G., and Yates, J. R. 3rd. (2004). A model for random sampling and estimation of relative protein abundance in shotgun proteomics. Anal. Chem. 76, 4193-4201.

Luttik, M.A., Overkamp, K.M., Kötter, P., de Vries, S., van Dijken, J.P., and Pronk, J.T. (1998). The Saccharomyces cerevisiae NDE1 and NDE2 genes encode separate mitochondrial NADH dehydrogenases catalyzing the oxidation of cytosolic NADH. J. Biol. Chem. 38, 24529-24534.

Maćasev, D., Whelan, J., Newbigin, E., Silva-Filho, M. C., Mulhern, T. D., and Lithgow, T. (2004). Tom22', an 8-kDa trans-site receptor in plants and protozoans, is a conserved feature of the TOM complex that appeared early in the evolution of eukaryotes. Mol. Biol. Evol. 21,1557-1564.

Mannella, C. A., Pfeiffer, D. R., Bradshaw, P. C., Moraru, I. I., Slepchenko, B., Loew, L. M., Hsieh, C. E., Buttle, K., and Marko, M. (2001). Topology of the mitochondrial inner membrane: dynamics and bioenergetic implications. IUBMB Life. 52, 93-100.

Mannella, C.A. (2008). Structural diversity of mitochondria: functional implications. Ann. N. Y. Acad. Sci. 1147,171-179.

Meisinger, C., et al. (2004). The mitochondrial morphology protein Mdm10 functions in assembly of the preprotein translocase of the outer membrane. Dev. Cell. 7, 61-71.

Meisinger, C., Pfanner, N., and Truscott, K. N. (2006). Isolation of yeast mitochondria. Methods. Mol. Biol. 313, 33-39.

Meisinger, C., et al. (2007). The morphology proteins Mdm12/Mmm1 function in the major beta-barrel assembly pathway of mitochondria. EMBO. J. 26, 2229-2239.

Messerschmitt, M., Jakobs, S., Vogel, F., Fritz, S., Dimmer, K. S., Neupert, W., and Westermann, B. (2003). The inner membrane protein Mdm33 controls mitochondrial morphology in yeast. J. Cell. Biol. 160, 553-564.

Minauro-Sanmiguel, F., Wilkens, S., and García, J.J. (2005). Structure of dimeric mitochondrial ATP synthase: novel F0 bridging features and the structural basis of mitochondrial cristae biogenesis. Proc. Natl. Acad. Sci. U S A. 35, 12356-12358.

Mick, D. U., Wagner, K., van der Laan, M., Frazier, A. E., Perschil, I., Pawlas, M., Meyer, H. E., Warscheid, B., and Rehling, P. (2007). Shy1 couples Cox1 translational regulation to cytochrome c oxidase assembly. EMBO J. 26, 4347-4358.

Mick, D. U., Vukotic, M., Piechura, H., Meyer, H. E., Warscheid, B., Deckers, M., and Rehling, P. (2010). Coa3 and Cox14 are essential for negative feedback regulation of COX1 translation in mitochondria. J. Cell. Biol. 191, 141-54.

Mick, D.U., Fox, T.D., and Rehling, P. (2011). Inventory control: cytochrome c oxidase assembly regulates mitochondrial translation. Nat. Rev. Mol. Cell. Biol. 2011. 1, 14-20.

Milenkovic, D., Gabriel, K., Guiard, B., Schulze-Specking, A., Pfanner, N., and Chacinska, A. (2007). Biogenesis of the essential Tim9-Tim10 chaperone com- plex of mitochondria: site-specific recognition of cysteine residues by the inter- membrane space receptor Mia40. J. Biol. Chem. 282, 22472-22480.

Mun, J. Y., Lee, T. H., Kim, J. H., Yoo, B. H., Bahk, Y. Y., Koo, H. S., and Han, S. S. (2010). Caenorhabditis elegans mitofilin homologs control the morphology of mitochondrial cristae and influence reproduction and physiology. J. Cell. Physiol. 224, 784-756. 
Muto, T., Obita, T., Abe, Y., Shodai, T., Endo, T., and Kohda, D. (2001) NMR identification of the Tom20 binding segment in mitochondrial presequences. J. Mol. Biol. 306, 137-43.

Neupert, W., and Herrmann, J. M. (2007). Translocation of proteins into mitochondria. Annu. Rev. Biochem. 76, 723-49.

Nijtmans, L. G., Henderson, N. S., and Holt, I. J. (2002). Blue Native electrophoresis to study mitochondrial and other protein complexes. Methods. 26, 327-34.

Nikolov, M., Stuetzer, A., Mosch, K., Krasauskas, A., Soeroes, S., Stark, H., Urlaub, H., and Fischle, W. (2011). Chromatin affinity purification and quantitative mass spectrometry defining the interactome of histone modification patterns. Mol. Cell. Proteomics. 10, M110.005371.

Odgren, P. R., Toukatly, G., Bangs, P. L., Gilmore, R., and Fey, E. G. (1996). Molecular characterization of mitofilin (HMP), a mitochondria-associated protein with predicted coiled coil and intermembrane space targeting domains. J. Cell. Sci. 109, 2253-2264.

Oka, T., Sayano, T., Tamai, S., Yokota, S., Kato, H., Fujii, G., and Mihara, K. (2008). Identification of a novel protein MICS1 that is involved in maintenance of mitochondrial morphology and apoptotic release of cytochrome c. Mol. Biol. Cell. 19, 2597-2608.

Olichon, A., Emorine, L. J., Descoins, E., Pelloquin, L., Brichese, L., Gas, N., Guillou, E., Delettre, C., Valette, A., Hamel, C. P., Ducommun, B., Lenaers, G., and Belenguer, P. (2002). The human dynaminrelated protein OPA1 is anchored to the mitochondrial inner membrane facing the inter-membrane space. FEBS Lett. 523, 171-176.

Olichon, A., Baricault, L., Gas, N., Guillou, E., Valette, A., Belenguer, P., and Lenaers, G. (2003). Loss of OPA1 perturbates the mitochondrial inner membrane structure and integrity, leading to cytochrome $\mathrm{c}$ release and apoptosis. J. Biol. Chem. 278, 7743-7746.

Ong, S. E., Blagoev, B., Kratchmarova, I., Kristensen, D. B., Steen, H., Pandey, A., and Mann, M. (2002). Stable isotope labeling by amino acids in cell culture, SILAC, as a simple and accurate approach to expression proteomics. Mol. Cell Proteomics. 1, 376-386.

Ong S. E., and Mann M. (2006) A practical recipe for stable isotope labeling by amino acids in cell culture (SILAC). Nat. Protoc. 1, 2650-2660.

Okamoto, K., and Shaw, J.M. (2005). Mitochondrial morphology and dynamics in yeast and multicellular eukaryotes. Annu. Rev. Genet. 39, 503-536.

Osman, C., Merkwirth, C., Langer, T. (2009). Prohibitins and the functional compartmentalization of mitochondrial membranes. J. Cell. Sci. 122, 3823-3830.

Pan, Y. (2011). Mitochondria, reactive oxygen species, and chronological aging: a message from yeast. Exp. Gerontol. 46, 847-852.

Paschen, S. A, Waizenegger, T., Stan, T., Preuss, M., Cyrklaff, M., Hell, K., Rapaport, D., and Neupert, W. (2003). Evolutionary conservation of biogenesis of beta-barrel membrane proteins. Nature. 426, 862-866.

Pfanner, N., and Geissler, A. (2001). Versatility of the mitochondrial protein import machinery. Nat. Rev. Mol. Cell. Biol. 2, 339-349.

Paumard, P., Arselin, G., Vaillier, J., Chaignepain, S., Bathany, K., Schmitter, J. M., Brèthes, D., and Velours, J. (2002a). Two ATP synthases can be linked through subunits $i$ in the inner mitochondrial membrane of Saccharomyces cerevisiae. Biochemistry. 41, 10390-10396.

Paumard, P., Vaillier, J., Coulary, B., Schaeffer, J., Soubannier, V., Mueller, D. M., Brèthes, D., di Rago, J. P., and Velours, J. (2002b). The ATP synthase is involved in generating mitochondrial cristae morphology. EMBO J. 21, 221-230. 
Pfanner, N., Tropschug, M., and Neupert, W. (1987). Mitochondrial protein import: nucleoside triphosphates are involved in conferring import-competence to precursors. Cell. 49, 815-823.

Peixoto, P. M., Grana, F., Roy, T. J., Dunn, C. D., Flores, M., Jensen, R. E., and Cam- po, M. L. (2007). Awaking TIM22, a dynamic ligand-gated channel for protein in- sertion in the mitochondrial inner membrane. J. Biol. Chem. 282, 18694-18701.

Rabl, R., Soubannier, V., Scholz, R., Vogel, F., Mendl, N., Vasiljev-Neumeyer, A., Körner, C., Jagasia, R., Keil, T., Baumeister, W., Cyrklaff, M., Neupert, W., and Reichert, A. S. (2009). Formation of cristae and crista junctions in mitochondria depends on antagonism between Fcj1 and Su e/g. J. Cell Biol. 185, 1047-1063.

Racker, E. A mitochondrial factor conferring oligomycin sensitivity on soluble mitochondrial ATPase. (1963). Biochem. Biophys. Res. Commun. 10, 435-439.

Rak, M., Tetaud, E., Godard, F., Sagot, I., Salin, B., Duvezin-Caubet, S., Slonimski, P. P., Rytka, J., and di Rago, J.P. (2007). Yeast cells lacking the mitochondrial gene encoding the ATP synthase subunit 6 exhibit a selective loss of complex IV and unusual mitochondrial morphology. J Biol Chem. 15, 1085310864 .

Rehling, P., Model, K., Brandner, K., Kovermann, P., Sickmann, A., Meyer, H. E., Kühlbrandt, W., Wagner, R., Truscott, K.N., and Pfanner, N. (2003). Protein inser- tion into the mitochondrial inner membrane by a twin-pore translocase. Science. 299, 1747-1751.

Reinders, J., Zahedi, R. P., Pfanner, N., Meisinger, C., and Sickmann, A. (2006). Toward the complete yeast mitochondrial proteome: multidimensional separation techniques for mitochondrial proteomics. J. Proteome. Res. 5, 1543-1554.

Reinhold, R., Bareth, B., Balleininger, M., Wissel, M., Rehling, P., and Mick, D. U. (2011). Mimicking a SURF1 allele reveals uncoupling of cytochrome c oxidase assembly from translational regulation in yeast. Hum. Mol. Genet. 20, 2379-2393.

Renken, C., Siragusa, G., Perkins, G., Washington, L., Nulton, J., Salamon, P., Frey, T. G. (2002). A thermodynamic model describing the nature of the crista junction: a structural motif in the mitochondrion. J. Struct. Biol. 138, 137-44.

Russ, W. P., and Engelman, D. M. (2000). The GxxxG motif: a framework for transmembrane helixhelix association. J. Mol. Biol. 296, 911-919.

Saddar, S., and Stuart, R. A. (2005). The yeast F(1)F(0)-ATP synthase: analysis of the molecular organization of subunit $\mathrm{g}$ and the importance of a conserved GXXXG motif. J. Biol. Chem. 280, 2443524442.

Saito, K., Chen, M., Bard, F., Chen, S., Zhou, H., Woodley, D., Polischuk, R., Schekman, R., and Malhotra V. TANGO1 facilitates cargo loading at endoplasmic reticulum exit sites. Cell. 136, 891-902.

Sanger, F., Nicklen, S., and Coulson, A. R. (1977). DNA sequencing with chain-terminating inhibitors. Proc. Natl. Acad. Sci U S A. 74, 5463-7.

Schägger, H, and von Jagow, G. (1987). Tricine-sodium dodecyl sulfate polyarcylamide gel electrophoresis for the separation of proteins from 1 to $100 \mathrm{kDa}$. Anal. Biochem. 166, 368-379.

Schägger, H., and von Jagow, G. (1991). Blue native electrophoresis for isolation of membrane protein complexes in enzymatically active form. Anal Biochem. 199, 223-31.

Schägger, H., and Pfeiffer, K. (2000). Supercomplexes in the respiratory chains of yeast and mammalian mitochondria. EMBO J 19, 1777-1783.

Scorrano, L., Ashiya, M., Buttle, K., Weiler, S., Oakes, S. A., Mannella, C. A., and Korsmeyer, S. J. 
(2002). A distinct pathway remodels mitochondrial cristae and mobilizes cytochrome c during apoptosis. Dev. Cell. 2, 55-67.

Seelert, H., Dani, D.N., Dante, S., Hauss, T., Krause, F., Schäfer, E., Frenzel, M., Poetsch, A., Rexroth, S., Schwassmann, H.J., Suhai, T., Vonck, J., and Dencher, N.A. (2009). From protons to OXPHOS supercomplexes and Alzheimer's disease: structure-dynamics-function relationships of energytransducing membranes. 6, 657-671.

Seelert, H., and Dencher, N.A. (2011). ATP synthase superassemblies in animals and plants: two or more are better. Biochim. Biophys. Acta. 9, 1185-1197.

Shevchenko, A., Tomas, H., Havlis, J., Olsen, J. V., and Mann, M. (2006). In-gel digestion for mass spectrometric characterization of proteins and proteomes. Nat. Protoc. 1, 2856-2860.

Sicheritz-Ponten T, Kurland CG, and Andersson SGE. (1998). A phylogenetic analysis of the cytochrome $\mathrm{b}$ and cytochrome $\mathrm{c}$ oxidase I genes support an origin of mitochondria from within the Rickettsiaceae. Biochem. Biophys. Acta. 1365, 545-551.

Sickmann, A., Reinders, J., Wagner, Y., Joppich, C., Zahedi, R., Meyer, H. E., Schönfisch, B., Perschil, I., Chacinska, A., Guiard, B., Rehling, P., Pfanner, N., and Meisinger C. (2003). The proteome of Saccharomyces cerevisiae mitochondria. Proc. Natl. Acad. Sci. USA. 100, 13207-13212.

Sideris, D.P., and Tokatlidis, K. (2007). Oxidative folding of small Tims is mediated by site-specific docking onto Mia40 in the mitochondrial intermembrane space. Mol. Microbiol. 65, 1360-1373.

Spannagel, C., Vaillier, J., Arselin, G., Graves, P.V., Grandier-Vazeille, X., and Velours, J. (1998). Evidence of a subunit 4 (subunit b) dimer in favor of the proximity of ATP synthase complexes in yeast inner mitochondrial membrane, Biochim. Biophys. Acta. 1414, 260-264.

Steed, P.R., and Fillingame, R.H. (2008). Subunit a facilitates aqueous access to a membrane embedded region of subunit c in Escherichia coli F1FO ATP synthase, J. Biol. Chem. 283, 12365-12372.

Stock, D., Leslie, A.G., Walker, J.E. (1999). Molecular architecture of the rotary motor in ATP synthase. Science. 5445, 1700-1705.

Stroh, A., Anderka, O., Pfeiffer, K., Yagi, T., Finel, M., Ludwig, B., and Schägger, H. (2004). Assembly of respiratory complexes I, III, and IV into NADH oxidase supercomplex stabilizes complex I in Paracoccus denitrificans. J. Biol. Chem. 6, 5000-5007.

Strauss, M., Hofhaus, G., Schröder, R. R., and Kühlbrandt, W. (2008). Dimer ribbons of ATP synthase shape the inner mitochondrial membrane. EMBO J. 27, 1154-1160.

Stuart, R.A. (2008). Supercomplex organization of the oxidative phosphorylation enzymes in yeast mitochondria. J. Bioenerg. Biomembr. 5, 411-417.

Stuart, R.A., and Rehling, P. (2008). Mitochondrial biogenesis: is an old dog still teaching us new tricks? Meeting on the Assembly of the Mitochondrial Respiratory Chain. EMBO. Rep. 1, 33-38.

Srere P. A. (1999). Heterologous protein-protein interactions-quinary structures.

Methods 19, 193.

Soubannier, V., Vaillier, J., Paumard, P., Coulary, B., Schaeffer, J., and Velours, J. (2002). In the absence of the first membrane-spanning segment of subunit 4(b), the yeast ATP synthase is functional but does not dimerize or oligomerize. J. Biol. Chem. 277, 10739-10745.

Szklarczyk, R., and Huynen, M.A. (2010). Mosaic origin of the mitochondrial proteome. Proteomics. 22, 4012-4024.

Takeda M., Vassarotti, A., and Douglas, M. G. (1985). Nuclear genes coding the yeast mitochondrial adenosine triphosphatase complex. Primary sequence analysis of ATP2 encoding the F1-ATPase beta- 
subunit precursor. J. Biol. Chem. 260, 15458-15465.

Thomas, D., Bron, P., Weimann, T., Dautant, A., Giraud, M. F., Paumard, P., Salin, B., Cavalier, A., Velours, J., and Brèthes, D. (2008). Supramolecular organization of the yeast F1Fo-ATP synthase. Biol. Cell. 100, 591-601.

Tsukihara, T., Aoyama, H., Yamashita, E., Tomizaki, T., Yamaguchi, H., Shinzawa-Itoh, K., Nakashima, R., Yaono, R., and Yoshikawa, S. (1996). The whole structure of the 13-subunit oxidized cytochrome c oxidase at 2.8 A. $272,1136-44$.

van der Laan, M., Hutu, D.P., and Rehling, P. (2010). On the mechanism of preprotein import by the mitochondrial presequence translocase. Biochim. Biophys. Acta. 6, 732-732.

Velours, J. and Arselin, G. (2000). The Saccharomyces cerevisiae ATP synthase. J Bioernerg Biomembr. 32, 383-90.

Velours, J., Dautant, A., Salin, B., Sagot, I., and Brèthes, D. (2009). Mitochondrial F1F0-ATP synthase and organellar internal architecture. Int. J. Biochem. Cell. Biol. 41,1783-1789.

Visser, W., Van Spronsen, E. A., Nanninga, N., Pronk, J. T., Gijs Kuenen, J., and van Dijken, J. P. (1995) Effects of growth conditions on mitochondrial morphology in Saccharomyces cerevisiae. Antonie Van Leeuwenhoek. 67, 243-253.

von der Malsburg, K., et al. (2011). Dual role of mitofilin in mitochondrial membrane organization and protein biogenesis. Dev Cell. 21, 694-707.

Vonck, J., and Schäfer, E. (2009). Supramolecular organization of protein complexes in the mitochondrial inner membrane. Biochim. Biophys. Acta. 1, 117-24.

Vogel, F., Bornhövd, C., Neupert, W., and Reichert, A. S. (2006). Dynamic subcompartmentalization of the mitochondrial inner membrane. J. Cell. Biol. 175, 237-247.

Vogel, F., Bornhövd, C., Neupert, W., and Reichert, A.S. (2006). Dynamic subcompartmentalization of the mitochondrial inner membrane. J Cell Biol 175, 237-47.

Wagner, K., Rehling, P., Sanjuán Szklarz, L. K., Taylor, R. D., Pfanner, N., and van der Laan, M. (2009). Mitochondrial F1Fo-ATP synthase: the small subunits e and g associate with monomeric complexes to trigger dimerization. J. Mol. Biol. 392, 855-861.

Walker, J. E., Lutter, R., Depuis,A. and Runswick, M. J. (1991). Identification of the subunits of F1F0ATPase from bovine heart mitochondria. Biochemistry. 30, 5369-5378.

Walker, E. (1994). The regulation of catalysis in ATP synthase. Curr. Opin. Struct. Biol. 4, 912-918.

Walker, J. E., Colinson, I. R., van Raaij, M. J. and Runswick, M. J. (1995). Structural analysis of ATP synthase from bovine heart mitochondria. Methods. Enzymol. 260, 163-190.

Wagner, K., Perschil, I., Fichter, C. D., and van der Laan, M. (2010). Stepwise assembly of dimeric $\mathrm{F}(1) \mathrm{F}(\mathrm{o})-\mathrm{ATP}$ synthase in mitochondria involves the small $\mathrm{F}(\mathrm{o})$-subunits $\mathrm{k}$ and i. Mol. Biol. Cell. 21, $1494-1504$.

Westermann, B. and Neupert, W. (2000). Mitochondria-targeted green fluorescent proteins: convenient tools for the study of organelle biogenesis in Saccharomyces cerevisiae. Yeast. 16, 1421-1427.

Wiedemann, N., Kozjak, V., Chacinska, A., Schönfisch, B., Rospert, S., Ryan, M. T., Pfanner, N., and Meisinger, C. (2003). Machinery for protein sorting and assembly in the mitochondrial outer membrane. $424,565-71$.

Wiedemann, N., Pfanner, N., and Rehling, P. (2006). Import of precursor proteins into isolated yeast mitochondria. Methods. Mol. Biol. 313, 373-83. 
Wittig, I., Karas, M., and Schägger, H. (2007). High resolution clear native electrophoresis for in-gel functional assays and fluorescence studies of membrane protein complexes. Mol Cell Proteomics. 6, 1215-1225.

Wittig, I., and Schägger, H. (2009). Supramolecular organization of ATP synthase and respiratory chain in mitochondrial membranes. Biochim. Biophys. Acta. 6, 672-680.

Wittig, I., Meyer B., Heide, H., Steger, M., Bleier, L., Wumaier, Z.,. Karas, M., and Schägger, H. (2010). Assembly and oligomerization of human ATP synthase lacking mitochondrial subunits a and A6L, Biochim. Biophys. Acta. 1797, 1004-1011.

Wu, Y., and Sha, B. (2006) Crystal structure of yeast mitochondrial outer membrane translocon member Tom70p. Nat. Struct. Mol. Biol. 13, 589-93.

Wurm, C. A., Suppanz, I. E., Stoldt, S., and Jakobs, S. (2010). Rapid FlAsH labelling in the budding yeast Saccharomyces cerevisiae. J. Microsc. 240, 6-13.

Xie, J., Marusich, M. F., Souda, P., Whitelegge, J., and Capaldi, R. A. (2007). The mitochondrial inner membrane protein mitofilin exists as a complex with SAM50, metaxins 1 and 2, coiled-coil-helix coiled-coil-helix domain-containing protein 3 and 6 and DnaJC11. FEBS Lett. 581, 3545-3549.

Yamaguchi, R., Andreyev, A., Murphy, A. N., Perkins, G. A., Ellisman, M. H., and Newmeyer, D. D. (2007). Mitochondria frozen with trehalose retain a number of biological functions and preserve outer membrane integrity. Cell Death Differ. 14, 616-624.

Yang, M., Jensen, R. E., Yaffe, M. P., Oppliger, W., and Schatz, G. (1988). Import of proteins into yeast mitochondria: the purified matrix processing protease contains two subunits which are encoded by the nuclear MAS1 and MAS2 genes. EMBO J. 7, 3857-3862.

Zheng, Y., Jung, M. K., and Oakley, B. R. (1991). Gamma-tubulin is present in Drosophila melanogaster and Homo sapiens and is associated with the centrosome. Cell. 65, 817-823.

Zick, M., Rabl, R., and Reichert AS. (2009). Cristae formation-linking ultrastructure and function of mitochondria. Biochim. Biophys. Acta. 1, 5-19. 


\section{ACKNOWLEDGMENTS}

First of all, I would like to thank Peter Rehling for the $\mathrm{PhD}$ opportunity in his lab, for his patience and continuous support. I would like to thank Markus Deckers for his supervision throughout the process. Special thanks goes to Melina Vucotic, Sasha Lytovchenko, and especially Fabian Ludewig for technical support and assistance. I would like to thank also all current and former members of the Rehling group for creating an amiable working environment (Christian Schulz, David Mick, Robert Reinhold, Bettina Bareth, Jan Dudek, Sven Dennerlein, Maria Levchenko, Jan Moritz Wuttke, Jonathan Melin, Isotta Lorenzi, Martina Balleininger, Mirjam Wissel, Cristine Vollmer, and Dana Hutu). I would also like to thank all other members of the Department of Biochemistry II especially Frau Engelmann (who makes me practice German) and Sarah Zafar (for her continuous health advice).

I would like to Reinhard Jahn and Markus Zweckstetter for their continuous support and insight in thesis committee meetings.

To all authors on the Alkhaja et al., 2012 publication: thank you for the fruitful collaboration! Thanks goes to Daniel Jans, Stefan Jakobs, Miroslav Nikolov, Henning Urlaub, Wolfgang Schliebs, and Dietmar Riedel. I would also like to Monika Raabe for her technical assistance.

Of course all of this would not be possible without the IMPRS Molecular Biology coordination office current and past members. Thank you Steffen Burkhardt, Kerstin Grüniger, and Ivana Jurik. I would also like to thank other members of the GGNB coordination office for all their hard work.

Studying and living in Göttingen has been interesting. It is "the best of times" and on some occasions "the worst of times". Yet, what makes it memorable is the Göttingen family of friends I have acquired over the last couple of years. I tried to look for a smart quote that I can write here, but words cannot simply express my gratitude. Thank you Derya, Natalia, Andrew, Alonso, Suenke, Jonas, Stephanie, Andrea, Matthieu, Sanaz, Ahmed, and Chris. You have always been there, through the bittersweet times. And of course: Sadeem, thank you for making me smile again, for inspiring me, for making Göttingen a happier place!

I would also like to thank my family back home (in Bahrain and Jeddah) who has supported me in all means possible. To my parents, I dedicate this to you. 


\section{CURRICULUM VITAE}

\section{Personal}

Born 02/03/1985, Manama, Bahrain

\section{Education}

Doctor of Philosophy, Molecular Biology

Georg-August University, Göttingen, Germany

$(2008-2012)$
Thesis title: The identification and characterization of Mio10 and MINOS1 as novel regulators of mitochondrial inner membrane organization

Supervisor: $\quad$ Prof. Dr. Peter Rehling

Dept. Biochemistry II

Georg August University Göttingen

University Medicine

Masters of Science; Molecular Biology, International Max Planck Research School, Georg-August University, Göttingen, Germany

(2007-2009)

Thesis title: Investigating the role of Mss51 in cytochrome c oxidase biogenesis and assembly

Supervisor: Prof. Dr. Peter Rehling Dept. Biochemistry II Georg August University Göttingen University Medicine

Bachelor of Science, Biochemistry w/ Molecular Biology (Industrial)

University of Leeds, Leeds, United Kingdom

(2003-2007)

Primary and Secondary Education

Bahrain Bayan School, Isa Town, Bahrain

(1991-2003) 